\title{
Development of a Bio-Inspired Whisker-like Sensor for Fluid Motion Tracking
}

\author{
A Dissertation Presented to the \\ Faculty of the School of Engineering and Applied Science \\ University of Virginia \\ In Partial Fulfillment \\ of the Requirements for the Degree of \\ Doctor of Philosophy \\ Mechanical and Aerospace Engineering
}

William C Eberhardt

December 2012 


\section{APPROVAL SHEET}

The dissertation is submitted in partial fulfillment of the requirements for the degree of

Doctor of Philosophy in Mechanical and Aerospace Engineering

William Craig Eberhardt

This dissertation has been read and approved by the Examining Committee:

\begin{tabular}{l}
\hline Benton Calhoun, Advisor \\
\hline Hossein Haj-Hariri, Chairman \\
\hline Colleen Reichmuth \\
\hline Eric Loth \\
\hline George Gillies
\end{tabular}

Accepted for the School of Engineering and Applied Science:

James H. Aylor, Dean

School of Engineering and Applied Science 


\begin{abstract}
Nature tackled the monitoring of surrounding fluid motion problem in many different, yet fundamentally similar, forms. Many terrestrial and aquatic species have developed hair and whisker sensory systems each specially suited to its environment and designed to observe the fluid motion surrounding the animal. Such an ability to passively monitor wakes of objects is of vital importance to surveillance and tracking in commercial, military, and scientific fields. This work is multidisciplinary combining efforts in the fields of biology, material science, electronics, and engineering to develop a whisker-like fluid motion sensor for tracking hydrodynamic wakes. The sensor inspiration comes primarily from the trichobothria of a wandering spider, Cupiennius salei, and vibrissae of harbor seals, Phoca vitulina.

The main contributions of the is work are: (1) the development of a kinematic viscoelastic model to describe trichobothria motion (2) experimental study of pinniped vibrissae to determine material properties and response to free and obstructed flows (3) evaluation of a microelectromechanical haircell sensor (4) development of a bioinspired whisker-like sensor for wake tracking and (5) development and testing of a Wake Information Detection and Tracking System (WIDTS).

A kinematic viscoelastic model is developed for the trichobothria of Cupiennius salei through force deflection tests. Force testing on trichobothria revealed the hair remains rigid under the loads and is supported by a membrane that provides the restoring and damping parameters of the kinematic system. A three parameter viscoelastic solid is in good agreement with the experimental results for all loading conditions of the hairs.

Tests conducted on whiskers of elephant seals Mirounga angustirostris, harbor seals Phoca vitulina, and California sea lions Zalophus californianus show that all
\end{abstract}


three are underdamped with natural frequencies under $100 \mathrm{~Hz}$. Force testing to examine the vibrissae material properties reveal the harbor seal whiskers to be the least stiff. Comparing the whisker's responses to flows, all three species demonstrate matching trends indicating at lower flow speeds the diameter perpendicular to the oncoming flow plays a larger role in the vibrissae motion than the undulatory morphology. Both the smooth and undulated vibrissae from the California sea lion and harbor seal show similar ranges in frequency and amplitude when in an unobstructed flow.

Analytical modeling of a haircell sensor show the sensor was limited to accurately capture fluid forces in a single direction and proved fragile. For wake tracking, placing the haircell sensor on the surface of a moving body would likely generate unwanted noise caused by the boundary layer flow blocking important flow information.

A whisker-like fluid motion sensor is developed pulling from the spider trichobothria's membrane sensor and the sizing criteria of vibrissae. Exciting the sensor with a sinusoidal signal and measuring the change in output voltage across a load resistor provide measurements of the sensor deflection in two orthogonal directions. The design proved to be robust and is able to monitor fluid speeds and directions and well as capture the frequency shedding of cylinder wakes.

Lastly, the sensor is packaged into arrays and a WIDTS system was developed for deployment on a trained harbor seal tracking a remote controlled submersible. Testing and numerical modeling show the WIDTS has a slight effect reducing the amplitude of wake signals reaching the seal, but the frequency information remained intact and well above the seal's threshold for capturing fluid motion. Preliminary field trials show the sensors are capable of detecting the sub's wake and indicate the side of the WIDTS the signal originated from. 


\section{Acknowledgements}

It is hard to fully put into words the time I have spent at UVA both as an undergrad and as a graduate student. I am truly thankful for the time spent, memories made, and friendships developed. They are years that I will always remember. First and foremost, with a heavy heart, I must thank Joseph Pepe Humphrey for his constant motivation, inspiration, and patience as I at times struggled through my Masters and Doctorate work. He will always be an inspiration to keep learning, observing, and questioning everything around us.

I would like to thank my colleagues: Yousef Shakhsheer who provided me with more knowledge regarding electronics and circuit design than I ever hoped to learn and was always there for help. Yousef was a vital resource in the development of the sensor theory and construction of the PCB. Without his help I would have been running around the pool with wires attached to the WIDTS. Chris Gregg for the development of the Java code to collect data from the WIDTS. Michael Landau for the tracking code and patience as I stumbled through it. John Paulus for the technical expertise in the sensor design and fabrication. Christin Murphy who was a great asset in the work with pinniped vibrissae and tolerated our terrible lab hours. Colleen Reichmuth and her group at the Long Marine Lab for all the help with Sprouts and enjoyable experiences during trips out west. Dr Bart-Smith's lab for the help with the milling machine, 3D printer, and advice on random ideas. Jonathan Stocking for the help building the water flume and effort getting the whisker sensor work started. Lewis Steva, Claude Mitchell, and Kevin Knight for help with machining and advice.

I would like to thank Ben Calhoun who inherited a mechanical engineer into his group and provided me the constant push to move forward with the project. I greatly appreciate all the advice, insight, conference trips, and freedom he gave me. I will be ever grateful for his guidance.

Finally, I would like to thank my family for their love, continuous support, and understanding through this journey. And last, Katharine for her unwaivering patience 
and love as I finish this chapter of my life. 


\section{Contents}

Abstract

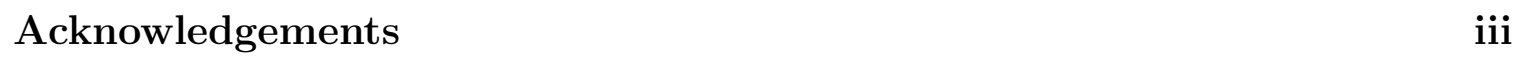

Nomenclature xiv

$\begin{array}{lll}1 & \text { Introduction } & 1\end{array}$

1.1 Inspiration . . . . . . . . . . . . . . . . . . . . . . . . . 2

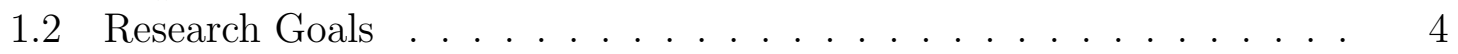

1.3 Research . . . . . . . . . . . . . . . . . . . . 5

$\begin{array}{lll}2 & \text { Background } & 7\end{array}$

2.1 Wake Hydrodynamics . . . . . . . . . . . . . . . . . . . . . . . 7

2.2 Biological Hair and Whisker Sensors . . . . . . . . . . . . . . . . . 8

2.2 .1 Fish . . . . . . . . . . . . . . . . . . . 8

2.2 .2 Spider . . . . . . . . . . . . . . . . . . . . . . . . . 9

2.2 .3 Seals . . . . . . . . . . . . . . . . . . . . . . . . . 10

2.3 Biologically Inspired Fluid Motion Sensors . . . . . . . . . . . . . . . 11

$\begin{array}{lll}3 & \text { Trichobothria Kinematics } & 14\end{array}$

3.1 Experimental Methods . . . . . . . . . . . . . . . . . . . . . . . 15

3.2 Experimental Results . . . . . . . . . . . . . . . . . . . . . . . 17

3.3 Viscoelastic Models . . . . . . . . . . . . . . . . . . . . . . . . . . . . 20

3.3 .1 Two Parameter Kelvin Solid Model . . . . . . . . . . . . . . . 22

3.3 .2 Three Parameter Solid Model . . . . . . . . . . . . . . . . . . 24

3.4 Multiple Regression . . . . . . . . . . . . . . . . . . . . . . . . . . . 24

3.5 Results . . . . . . . . . . . . . . . . . . . . . . . . . . . 27

3.6 Discussion . . . . . . . . . . . . . . . . . . . . . . . . . . . . . . . 28

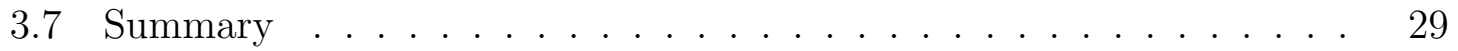

\begin{tabular}{|lll}
\hline & Vibrissae Study & 31
\end{tabular}

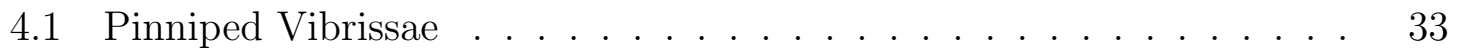

4.2 Specimen Preparation . . . . . . . . . . . . . . . . . . . . . . . 34

4.3 Experimental Methods . . . . . . . . . . . . . . . . . . . . . . . . . . 36

4.3 .1 Material Testing . . . . . . . . . . . . . . . . . . . . . . . 36

4.3 .2 Flume Study . . . . . . . . . . . . . . . . . . . . . . 37 
4.4 Theoretical Shedding Frequencies . . . . . . . . . . . . . . . . . . . . 40

4.5 Vibrissae Properties. . . . . . . . . . . . . . . . . . . . . . . . . . . . . . . . . . . . . .

4.6 Free Flow Results . . . . . . . . . . . . . . . . . . . . . . . . . . . . . 46

4.7 Obstructed Flow Results $\ldots \ldots \ldots$

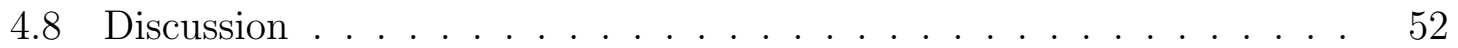

4.9 Summary . . . . . . . . . . . . . . . . . . . . . . 55

5 Haircell Sensor Modeling $\quad 56$

5.1 Cantilever Fluid Motion Sensor . . . . . . . . . . . . . . . . . 57

5.2 Modeling Sensor. . . . . . . . . . . . . . . . . . . . 58

5.2 .1 Simplification . . . . . . . . . . . . . . . . 58

5.2 .2 Governing Equations . . . . . . . . . . . . . . . . . . . . . . 59

$5.2 .3 \quad$ Finite Difference Solution . . . . . . . . . . . . . . . . . . . . 62

5.3 Results . . . . . . . . . . . . . . . . . . . 64

5.4 Validation . . . . . . . . . . . . . . . . . . . . . . . . . . . . . . . . . . .

5.5 Discussion $\ldots \ldots \ldots \ldots$. . . . . . . . . . . . . . . . . . . . . . . . . . 72

5.6 Summary . . . . . . . . . . . . . . . . . . . . . . 74

6 Bio-Whisker Sensor Development $\quad 75$

6.1 Whisker-like Sensor Designs . . . . . . . . . . . . . . . . . 76

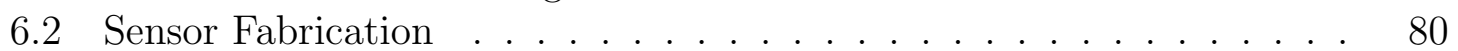

6.3 Sensor Prototype . . . . . . . . . . . . . . . . . . . . . . . . 81

6.4 Whisker-like Sensor Development . . . . . . . . . . . . . . . . . . . . 84

6.4 .1 Discussion of the Prototype . . . . . . . . . . . . . . . . . 84

6.4 .2 Sensor Excitation and Measurement. . . . . . . . . . . . . . . 86

6.4 .3 Revision 2 . . . . . . . . . . . . . . . . . 87

6.4 .4 Revision 3 . . . . . . . . . . . . . . . . . . . . . . 91

6.4 .5 Sensor Theory . . . . . . . . . . . . . . . . . . . 97

6.4 .6 Revision 4 . . . . . . . . . . . . . . . . . . . 101

6.5 Wake Measurement and Detection . . . . . . . . . . . . . . . . . . 103

6.6 Discussion . . . . . . . . . . . . . . . . . . . . . . . . . . . . . . . . . 108

6.7 Summary $\ldots \ldots \ldots \ldots$. . . . . . . . . . . . . . . . . . . 109

$\begin{array}{lll}7 & \text { Sensor Array Development and Deployment } & 110\end{array}$

7.1 WIDTS Development . . . . . . . . . . . . . . . . . . . . . . . . . 111

7.1 .1 Design Parameters . . . . . . . . . . . . . . . . . . . . . . . . . . 111

7.1 .2 Location and Attachment . . . . . . . . . . . . . . . . . . . . 113

$7.1 .3 \quad$ Design Evaluation. . . . . . . . . . . . . . . . . . . . . . 116

7.1 .4 Construction . . . . . . . . . . . . . . . . . . . . . 123

7.1 .5 Data Collection . . . . . . . . . . . . . . . . . 125

7.2 Chase Object . . . . . . . . . . . . . . . . . . . . . . . . . 125

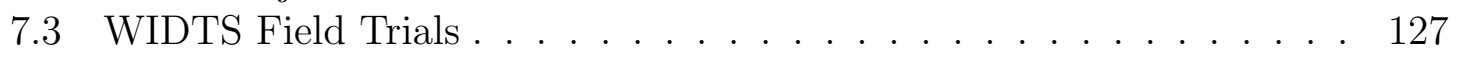

7.3 .1 Experimental Methods . . . . . . . . . . . . . . . . . . . 127

7.3 .2 RC Submarine Detection . . . . . . . . . . . . . . . . . . . . . 129

7.3 .3 RC Submarine Tracking . . . . . . . . . . . . . . . . . . 130 


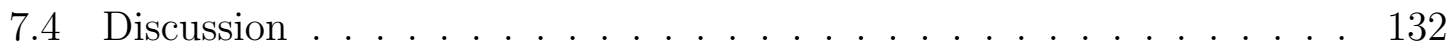

7.5 Summary . . . . . . . . . . . . . . . . . . . . . 134

8 Conclusions and Future Work 135

\begin{tabular}{|lr}
\hline A Propagation of Error & 148
\end{tabular}

\begin{tabular}{ll}
\hline B Beam Coding & 149
\end{tabular}

B.1 Matlab code for the explicit Runge-Kutta finite difference method . . 149

B.2 Matlab code for the implicit Crank-Nicolson finite difference method. 155

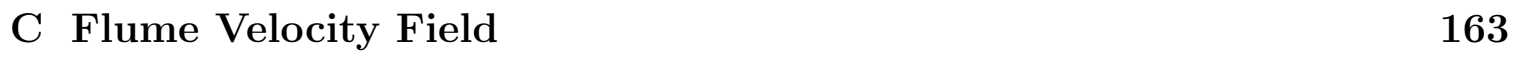




\section{List of Figures}

1.1 Cupiennius salei jumping toward tethered fly from sequence of video frames [1]. . . . . . . . . . . . . . . . . . . . . . 2

1.2 Harbor seal tracking submersible from sequence of video frames [2] . . 3

2.1 Schematic of lateral line canal (left) and a neuromast (right) located between two pores [3]. . . . . . . . . . . . . . . 9

2.2 (a)Cupiennius salei with trichobothria locations marked with yellow arrows. (b)Close-up of three trichobothria [4]. . . . . . . . . . . 10

3.1 Hair parameters. Only the pivoting regime is investigated, region D [4] 16

3.2 Comparison of results (a) before shaving and (b) after [4]. . . . . . . 17

3.3 (a) SEM micrograph of trichobothria tip showing microtrichs around the main hair shaft. (b) shortened trichobothria with partially removed

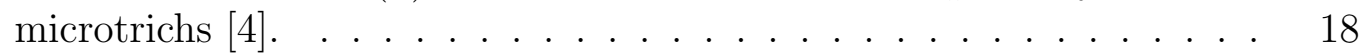

$3.4 \quad 114.5 \mu \mathrm{m}$ Trichobothria deflection measurement obtained at an angular velocity of $0.029 \mathrm{rad} / \mathrm{s}$. (a) Load-deflection curve (b) enlarged initial region. The dashed line represents the minimum angle necessary to elicit a nervous response from the spider [5] [4]. . . . . . . . . . . . . 19

3.5 Angular velocity dependence of the force-displacement results from 12 tests. (a) Hair pivots more at lower angular velocities. (b) Work required to reach physiological threshold $(0.01 \mathrm{deg}) \quad$. . . . . . . . . . 20

3.6 Comparison of measurements (circles) and empirical fits of (a) torque (b) the torque time rate of change . . . . . . . . . . . 21

3.7 Spring and dashpot representation of the two parameter model. . . . 23

3.8 Spring and dashpot representation of the three parameter model . . . 23

\begin{tabular}{ll}
3.9 & Comparison of experimental data and predicted torques from the vis- \\
\hline & coelastic models $\ldots \ldots \ldots \ldots \ldots \ldots$
\end{tabular}

4.1 Harbor seal (left) and California sea lion (right) vibrissae in erect po-

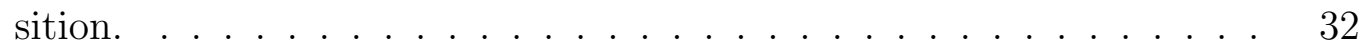

4.2 Closeup images of smooth vibrissae of California sea lion (top) and undulated vibrissae of elephant seal (middle) and harbor seal (bottom). 33

4.3 California sea lion vibrissae mounted for testing. . . . . . . . . . . . . 34

$4.4 \quad$ Harbor seal whisker mounted for a force-displacement test. . . . . . . 36

4.5 Rolling Hills water flume used to test vibrissae response to varying flow conditions. . . . . . . . . . . . . . . . . . . . . . . . . . . 38 
4.6 Design of tests in water flume [6]. . . . . . . . . . . . . . . . . . . . . 39

4.7 Force displacement plot for pinniped vibrissae. Forces applied $2 \mathrm{~cm}$ above the whisker base with displacements up to $4 \mathrm{~mm}$. . . . . . . . . 42

4.8 Force displacement plot along a single harbor seal vibrissae. Forces are applied 1,2 , and $5.6 \mathrm{~cm}$ from the base. . . . . . . . . . . . . 43

4.9 Free vibration of California sea lion vibrissae specimen C65. . . . . . 45

4.10 Frequency response of free vibration of California sea lion vibrissae specimen C65. . . . . . . . . . . . . . . . . . . . 45

4.11 Frequency response of free vibration for California sea lion (top), elephant seal (middle) and harbor seal (bottom) for 0 degree (left), 45 degree (middle), and 90 degree (right) orientations [6]. . . . . . . . . 47

4.12 Average peak frequency for each orientation . . . . . . . . . . . 48

4.13 Harbor seal whisker behind $2 \mathrm{~cm}$ cylinder with laser vibrometer measuring at midpoint. . . . . . . . . . . . . . . . . . . . . 49

4.14 Harbor seal (left) and California sea lion (right) in free flow (blue) and in presence of $1 \mathrm{~cm}$ cylinder (red) in a $5.08 \mathrm{~cm} / \mathrm{s}$ flow. Note the emergence of a $1 \mathrm{~Hz}$ peak in the cylinder wake case (red). . . . . . . . 50

4.15 Peak frequencies detected and theoretical shedding frequencies of a cylinder wake for harbor seal and California sea lion vibrissae. . . . . 51

4.16 Nondimensional results of vibrissae detection of cylinder wakes. . . . 52

5.1 Scanning electron micrograph of artificial haircell sensor [7]. . . . . . 57

5.2 Simplification of haircell sensor to a cantilever beam fixed and one end and with a concentrated mass and moment acting on the free end. . . 58

5.3 Resulting drag dominated moment from a fluid motion of $25 \mathrm{~Hz}$. . . . $\quad 65$

5.4 Resulting added mass influenced moment from a fluid motion of $250 \mathrm{~Hz} .65$

5.5 Dynamic results for the $250 \mathrm{~Hz}$ simulation. The dashed line represents the cantilever tip displacement. The bottom plot shows the cantilever at its maximum deflection (magnitude). . . . . . . . . . . . 66

5.6 Maximum haircell tip deflections for the base parameters for frequencies from 25 to $250 \mathrm{~Hz}$ and $u^{\prime} / U$ from 0.01 to 0.25 . . . . . . . . . . . 67

5.7 Maximum haircell tip deflections for doubling the diameter for frequencies from 25 to $250 \mathrm{~Hz}$ and $u^{\prime} / U$ from 0.01 to 0.25 . . . . . . . . . . . 68

5.8 Maximum haircell tip deflections for halving the thickness for frequencies from 25 to $250 \mathrm{~Hz}$ and $u^{\prime} / U$ from 0.01 to 0.25 . . . . . . . . . . . 69

5.9 Maximum haircell tip deflections vs frequency showing design changes,

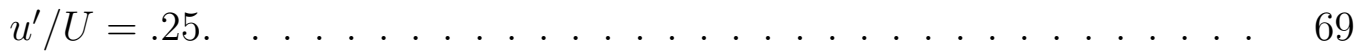

5.10 Haircell sensor response to an oscillating flow with $0.1 \mathrm{~m} / \mathrm{s}$ flow magnitude oscillating at $200 \mathrm{~Hz}$. The dashed line represents the cantilever tip displacement. The bottom plot shows the cantilever at its maximum deflection (magnitude). . . . . . . . . . . . . . 70

5.11 Haircell sensor response vs frequency to an oscillating flow of amplitude

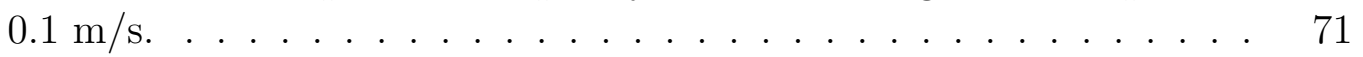

5.12 Haircell sensor response to an oscillating flow [8]. . . . . . . . . . . . 72

5.13 Haircell sensor response to an oscillating flow [8] . . . . . . . . . . 73 
6.1 Basic sensor design ideas: Capacitor measurement sensors of Barbier el al [9] (left) and modified capacitance sensor (middle) and strain measurement sensor (right). . . . . . . . . . . . . . . 77

6.2 Whisker-like sensor with strain gauges embedded in PDMS. . . . . . 78

6.3 Side and top view schematics of whisker-like cone-in-cone sensor design. 79

6.4 First whisker-like sensor prototype. . . . . . . . . . . . . . . 82

6.5 Force-capacitance measurement of each quadrant in the prototype sensor in compression [10]. . . . . . . . . . . . . . . . . 83

6.6 Force-capacitance measurement of each quadrant in the prototype sensor in separation [10]. . . . . . . . . . . . . . . . . . . . 83

6.7 Prototype sensor output (quadrant 3) in water flow [11]. . . . . . . . 84

6.8 Revision 1 signal increases with frequency . . . . . . . . . . . . . 87

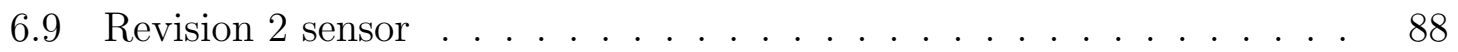

6.10 Revision 2 signal range for $330 \Omega$ load and input of $10 \mathrm{MHz}$ with 6.58

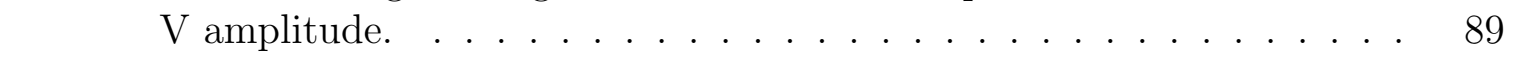

6.11 Revision 2 signal degradation . . . . . . . . . . . . . . . . . . . 90

6.12 Corrosion on copper plates. . . . . . . . . . . . . . . . . . . . . . . . 90

6.13 Whisker-like sensor revision three . . . . . . . . . . . . . . . . . . 91

6.14 Wire effects on the sensor gain range. . . . . . . . . . . . . . . . . . 93

6.15 Improvement in gain range of revision compared to the prototype. . . 93

6.16 Gain range as a function of input signal frequency and fluid in the gap. 94

6.17 Peak voltage output of quadrant facing water flow direction as the flow velocity increases. . . . . . . . . . . . . . . . 95

6.18 Peak values of sensor output measuring the cross stream deflections behind a $2 \mathrm{~cm}$ diameter cylinder. . . . . . . . . . . . . . . . . . 96

6.19 Cross stream quadrant frequency response to $5.02 \mathrm{~Hz}$ cylinder wake. . 96

6.20 Degradation of waterproofing layer. Outer cone surface before testing (left) and after three months (right) _. . . . . . . . . . . . 97

6.21 Schematic of the circuit model to estimate sensor performance. . . . . 98

6.22 Comparison of gain between revision three and the theory for a) compression b)separation $\ldots \ldots \ldots \ldots . \ldots \ldots 9 . \ldots \ldots$

6.23 Theoretical gain range of the sensor as a function of dielectric material in the gap. . . . . . . . . . . . . . . . . 100

6.24 Contour plot of gain range for input frequency and load in distilled

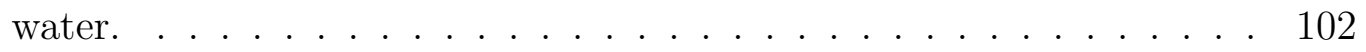

6.25 Revision four pictured without PDMS membrane. . . . . . . . . . . . 102

6.26 Experimental test of revision four compared to the model without parasitic capacitances with a $100 \mathrm{~Hz}$ input frequency showing gain range as a function of load. . . . . . . . . . . . . . . . . . . . . 103

6.27 Revision four in water flume behind stationary cylinder. Right: Side view of sensor 25 body lengths behind $2 \mathrm{~cm}$ cylinder (in red box). Left: View looking upstream of sensor behind $8.9 \mathrm{~cm}$ cylinder. . . . . . . . 104

6.28 Sensor response to a $8.9 \mathrm{~cm}$ cylinder $51 \mathrm{~cm}$ upstream in a $46 \mathrm{~cm} / \mathrm{s}$ flow. Top: raw data from the cross stream quadrant (left) and quadrant facing the flow (right). Bottom: frequency response of measured data. 105 
6.29 Frequency response of cross stream quadrant. Left: $2 \mathrm{~cm}$ cylinder in a $61 \mathrm{~cm} / \mathrm{s}$ flow. Right: $8.9 \mathrm{~cm}$ cylinder in a $15 \mathrm{~cm} / \mathrm{s}$ flow. . . . . . . . . 106

6.30 Sensor peak output as a $2 \mathrm{~cm}$ cylinder traverses the tank in a 45.7 $\mathrm{cm} / \mathrm{s}$ flow. . . . . . . . . . . . . . . . . 107

6.31 Sensor peak output as a $6 \mathrm{~cm}$ cylinder traverses the tank in a 45.7 $\mathrm{cm} / \mathrm{s}$ flow. . . . . . . . . . . . . . . . . . . 107

6.32 Sensor peak output as a $8.9 \mathrm{~cm}$ cylinder traverses the tank in a 45.7 $\mathrm{cm} / \mathrm{s}$ flow. . . . . . . . . . . . . . . . 108

7.1 Ring array of whisker-like fluid motion sensors. . . . . . . . . . . . . . 112

7.2 Torpedo shaped WIDTS in $0.5 \mathrm{~m} / \mathrm{s}$ flow with red dye showing water flow over the WIDTS surface. . . . . . . . . . . . . . . . . . . . 113

7.3 Modified ellipsoid nose section blending to the octagonal ring array. . 113

7.4 Possible WIDTS locations. WIDTS models attached to Sprouts on top of his head (left), below (middle), and held in his mouth (right). . . . 114

7.5 WIDTS attachment devices. Left: hat to locate WIDTS above Sprouts' head. Middle: early bite plate to hold WIDTS in front of Sprouts' muzzle. Right: bite plate variations. . . . . . . . . . . . . . . . 115

$7.6 \quad$ WIDTS mock up with finalized bite plates for training. . . . . . . . . 115

7.7 Harbor seal head modeling being printed. . . . . . . . . . . . . . . . 117

7.8 Harbor seal head with WIDTS for PIV testing. . . . . . . . . . . . . 117

7.9 Average vector field from PIV measurement. Black outline indicates the edge of the seal head and corner of the WIDTS. . . . . . . . . . . 118

7.10 Steady state velocity contour plot of $0.5 \mathrm{~m} / \mathrm{s}$ flow around seal head with and without WIDTS. . . . . . . . . . . . . . . . . . . . 120

7.11 Velocity vectors of $0.5 \mathrm{~m} / \mathrm{s}$ steady state flow around seal head with and without WIDTS. . . . . . . . . . . . . . . . 121

7.12 Location of near and far data points monitoring cylinder wake flow. . 121

7.13 Velocity magnitude monitored by the near and far locations in the presence of a cylinder wake with (right) and without (left) the WIDTS obstructing the flow ahead of the harbor seal head. . . . . . . . . . . 122

7.14 Frequency response of velocity monitored by near and far locations in the presence of a cylinder wake with (right) and without (left) the WIDTS obstructing the flow ahead of the harbor seal head. . . . . . . 123

7.15 Wire frame schematic of the nose section (left) and a sensor array ring

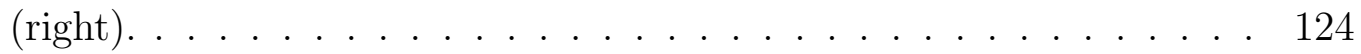

7.16 Exploded view of the final WIDTS assembly. . . . . . . . . . . . . 125

7.17 Chase vehicle in flume. . . . . . . . . . . . . . . . . . . . . 126

7.18 Contour plot of averaged flow field behind Neptune submarine. . . . . 127

7.19 Blindfolded Sprouts chasing a $6 \mathrm{~cm}$ ball during training. . . . . . . . 128

7.20 Side and front view of Sprouts holding WIDTS for testing. . . . . . . . 128

7.21 Data from sweeping WIDTS behind RC submarine. Red lines indicate when the WIDTS was directly behind the sub. Top plot shows the WIDTS motion, middle and bottom plots show rear quadrant data for the right and left sensors with circles around wake detection spikes. . 129 
7.22 Track of the sub (yellow) and path of the WIDTS held in Sprouts' mouth $(\mathrm{red}) . \ldots \ldots \ldots \ldots \ldots 131$

7.23 Snapshots of Sprouts chasing the sub at 27.2 seconds (left) and 29 seconds (right). The residual submarine wake location is circled in red. 131

7.24 Left and right sensor data with red lines marking peaks indicating submarine wake detection. . . . . . . . . . . . . . . . . 132

C.1 Calibration of motor speed $(\mathrm{Hz})$ to water velocity $(\mathrm{in} / \mathrm{s}) \quad$. . . . . . . 164

C.2 Countour plot of flow velocity magnitude for $41.7 \mathrm{~cm} / \mathrm{s}$ flow. . . . . . 164

C.3 Countour plot of flume turbulence for $41.7 \mathrm{~cm} / \mathrm{s}$ flow $\quad \ldots . . .165$ 


\section{List of Tables}

3.1 Data used to determine kinematic mechanics of trichobothria. Data points are from 81 trials . . . . . . . . . . . . . . . . 20

3.2 RMS values for data used to determine kinematic mechanics of trichobothria. Data points are used to determine the confidence intervals of the viscoelastic parameters. . . . . . . . . . . . . . . . . . 22

4.1 Vibrissae length measurements . . . . . . . . . . . . . . . . . . . 35

4.2 Predicted shedding frequencies of vibrissae at 0 and 90 degree orienta-

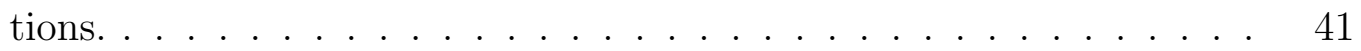

4.3 Characteristic vibration properties of vibrissae . . . . . . . . . . . . 46

4.4 Vibrissae motion in $0.5 \mathrm{~m} / \mathrm{s}$ free stream flow. . . . . . . . . . . . . . . 48

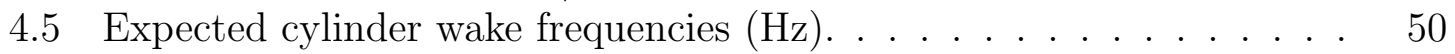

$5.1 \quad$ Parameters for haircell sensor modeling . . . . . . . . . . . . . . . . . 62

6.1 Dimensions of prototype whisker-like sensor . . . . . . . . . . . . . . 81 


\section{Nomenclature}

$\ddot{\theta} \quad$ angular acceleration

$\Delta t \quad$ timestep

$\Delta x \quad$ distance between nodes

$\delta \quad$ logarithmic decrement

$\dot{\theta} \quad$ angular velocity

$\dot{T} \quad$ time rate of change of torque

$\epsilon \quad$ permittivty of free space

$\nu \quad$ kinematic viscosity

$\omega \quad$ frequency

$\omega_{d} \quad$ damped natural frequency

$\omega_{n} \quad$ natural frequency

$\rho_{\text {beam }}$ beam density

$\rho_{\mathrm{H}_{2} \mathrm{O}}$ density of water

$\rho_{h} \quad$ trichobothria density

$\rho_{\text {post }}$ SU-8 epoxy post density

$\theta \quad$ displacement angle

$\zeta \quad$ damping ratio

$A_{\text {beam }}$ beam cross sectional area

$C_{\text {base }}$ parasitic capacitance from outer cone

$C_{\text {cone }}$ parasitic capacitance from inner cone

$C_{D} \quad$ coefficient of drag 
$C_{\text {edge }}$ total capacitance

$C_{\text {gap }}$ inclined plate capacitance

$C_{\text {gap }}$ total capacitance

$C_{\text {total }}$ total capacitance

$C_{\text {wire }}$ wire capacitance

$F_{\text {drag }}$ drag force

$F_{\text {mass }}$ added mass force

$R_{2 p} \quad$ damping constant of Kelvin solid model

$R_{3 p} \quad$ damping constant of 3 parameter solid model

$R_{\text {gap }}$ equivalent resistance from fluid in sensor

$R_{\text {load }}$ sensor resistive load

$R_{\text {wire }}$ wire resistance

$S_{3 p}^{\prime} \quad$ second torsional restoring constant of 3 parameter solid model

$S_{2 p} \quad$ torsional restoring constant of Kelvin solid model

$S_{3 p} \quad$ torsional restoring constant of 3 parameter solid model

$S_{v i b} \quad$ spring constant of vibrissae

$U_{\mathrm{H}_{2} \mathrm{O}}$ velocity of water

$U_{\text {post }}$ velocity of post

$U_{\text {rel }}$ velocity of whisker relative to fluid velocity

$V_{\text {post }}$ volume of post

$X_{i} \quad$ amplitude of vibration

A area

a location of point load

C capacitance

D post diameter

d diameter

E Young's modulus of elasticity 


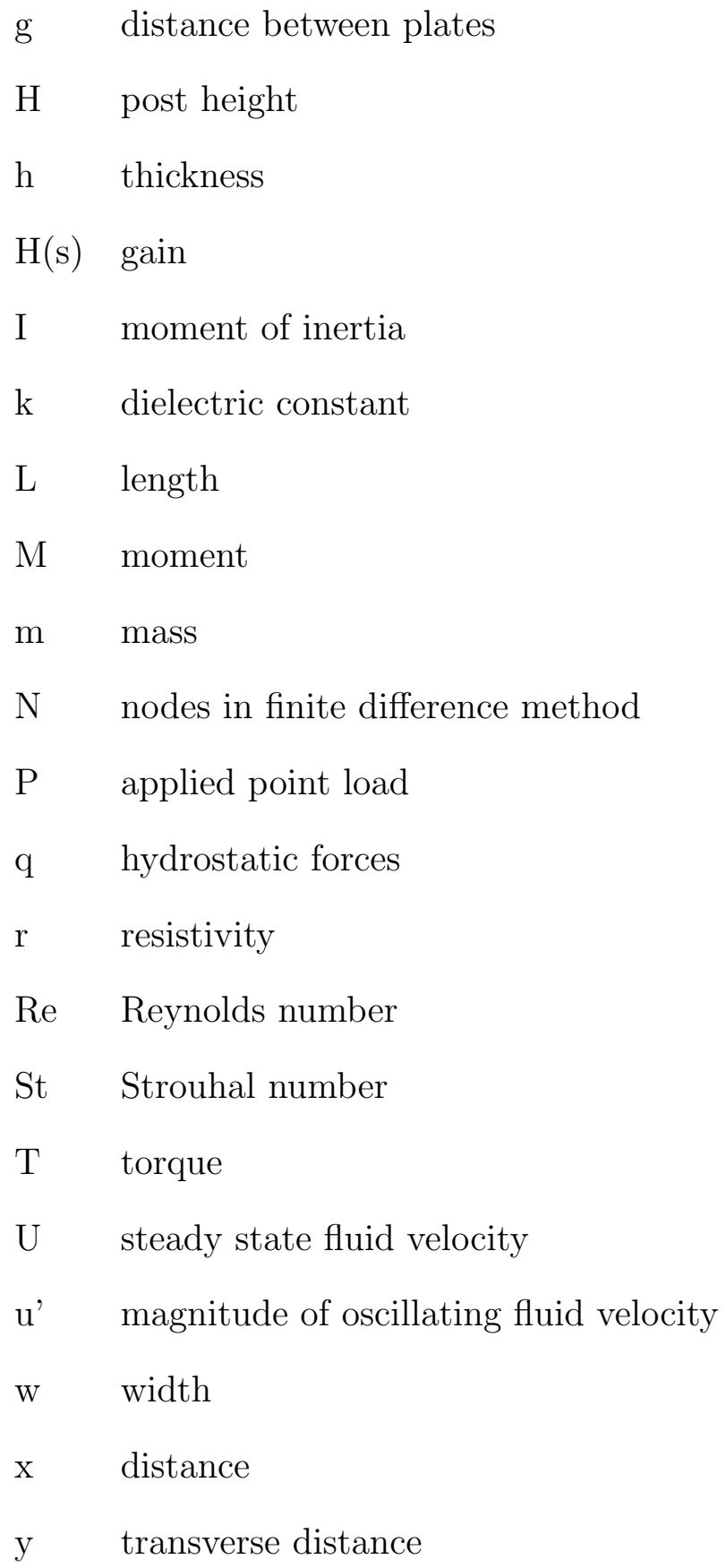




\section{Chapter 1}

\section{Introduction}

The ability to navigate a fluid environment using only hydrodynamic information without visual or acoustic cues represents a technical challenge with implications for surveillance and tracking in commercial, military, and scientific fields. Current autonomous underwater vehicles (AUVs) often rely on active sonar and video for object detection [12]. Object detection and localization is often limited by murky water with limited visibility. Sonar requires an AUV to emit an acoustic signal and listen to time delays in the signal return to generate an image of the surrounding area [13] [14]. The distance of detection in sonar is limited by the frequency of the signal [13]. Lower frequencies travel greater distances than higher frequencies. Scattering and fluid characteristics influence the sonar resolution. Since a signal must be transmitted to the surrounding area, the use of sonar may also alert others of an AUV's presence. Thus a passive means of detecting and tracking an underwater object would assist in areas where stealth is important. Therefore a sensor capable of directly monitoring the surrounding fluid motion to detect signatures is needed. This work concentrates on taking inspiration from nature's solution to fluid motion sensing and using it to develop a biologically inspired whisker-like sensor capable of passively capturing the motion of the surrounding fluid from hydrodynamic wakes. The sensor developed here must be capable of monitoring fluid speed and direction. 

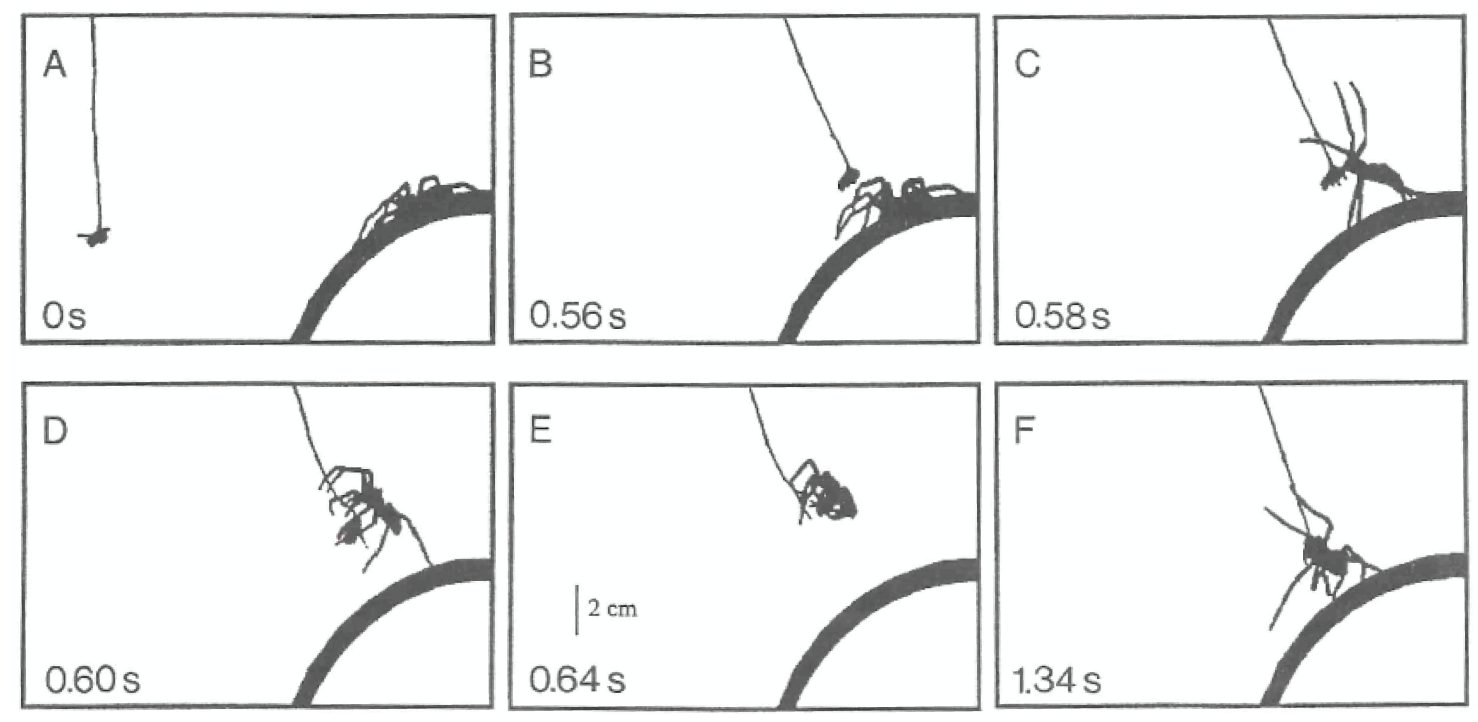

Figure 1.1: Cupiennius salei jumping toward tethered fly from sequence of video frames [1].

\subsection{Inspiration}

Nature tackled the monitoring of surrounding fluid motion problem in many different, yet fundamentally similar, forms. Many terrestrial and aquatic species have developed hair and whisker sensory systems each specially suited to its environment and designed to observe the fluid motion surrounding the animal. These biological hair cells and whiskers exhibit a wide array of dimensions depending on their application [15]. It is advantageous for an animal to gather this information in such a manner that does not alert its prey or a predator. Fish obtain hydrodynamic information through a distribution of internal and external neuromasts [16]. The blind cave fish use neuromasts within a lateral line (internal) to regulate its distance from a wall while swimming [17]. These hair sensors are up to $400 \mu \mathrm{m}$ in length [18]. Crickets use low frequency response hairs on their back to detect approaching predators [19]. The cricket hair sensors range from 30-1500 $\mu \mathrm{m}$ in height and 1-9 $\mu \mathrm{m}$ in diameter [20]. They can detect airflows as low as $0.01 \mathrm{~mm} / \mathrm{s}$ [21] generating deflections of the hair of less than $10^{-4} \mathrm{rad}[22$. Spiders are capable of detecting a passing insect and leaping into the air to catch its prey [23]. A sequence of video frames in Figure 1.1 shows a 


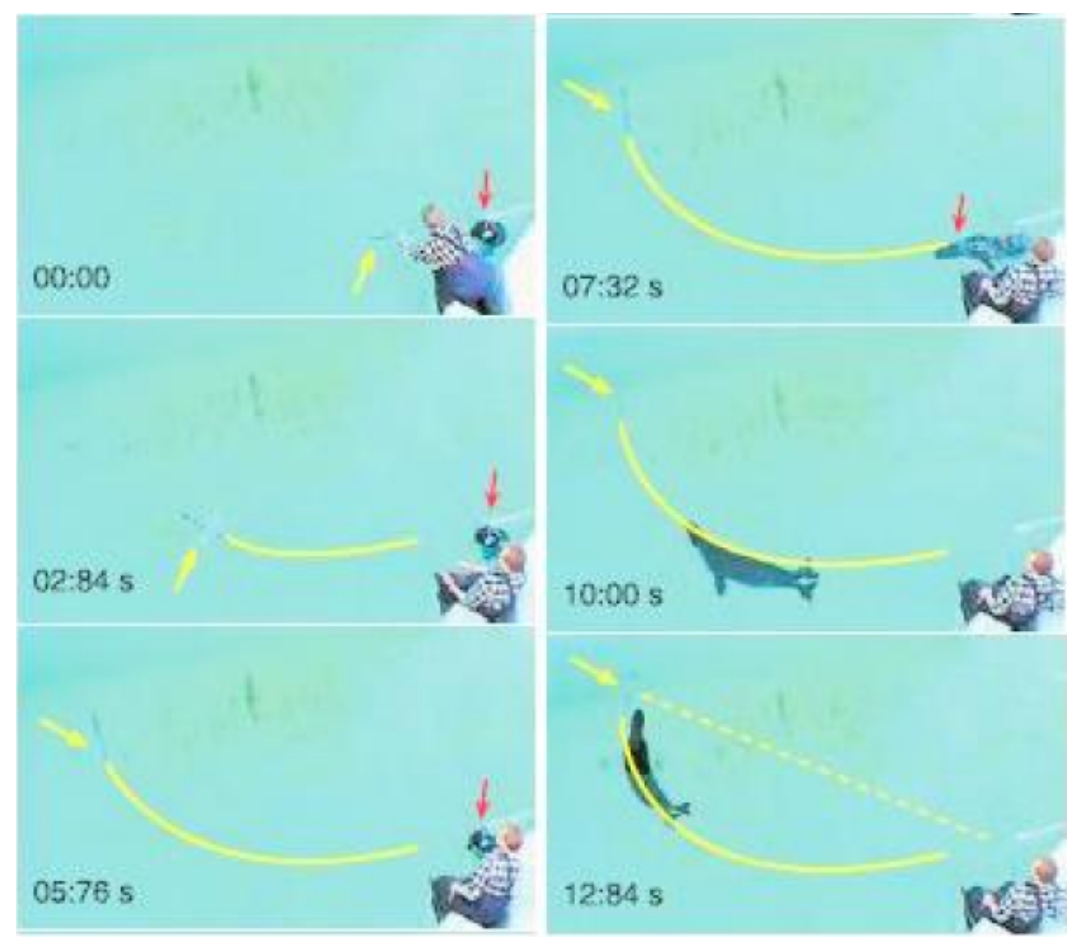

Figure 1.2: Harbor seal tracking submersible from sequence of video frames [2]

blinded wandering spider leaping from a leaf to capture a tethered fly. The position of the fly was determined solely by hair motion sensors on its legs. Mammalian inner ear hair cells range from 2-8 $\mu \mathrm{m}$ tall and 0.1-0.3 $\mu \mathrm{m}$ in diameter [24] and have nanometer resolution [25]. Harbor seals are capable of detecting minute fluid movements as low as $245 \mu \mathrm{m} / \mathrm{s}$ [26]. The harbor seal is capable of tracking an underwater object using only their whiskers as shown in Figure 1.2. As flow sensors these hair and whisker sensors exhibit high sensitivity to both flow magnitude and direction. Both traits are key factors in development of bio-inspired sensors for real world applications such as wake detection and object tracking. This leads to several overarching questions: (1) What allows these systems to have such high sensitivities? (2) What properties are most important to replicate? (3) How do we design and build an artificial sensor incorporating these features? 


\section{$1.2 \quad$ Research Goals}

The goal of this research is to increase an understanding of biological fluid motion sensors and in turn develop a fluid motion sensor array to be carried by a harbor seal to track a RC submersible. Specifically hair-like sensors, trichobothria, from Cupiennius salei and whiskers, or vibrissae, from pinniped seals are investigated with an effort to pull material properties and develop the mechanics of motion of the sensory system while observing their ability to detect hydrodynamic stimuli. It would be difficult to directly match the viscoelastic properties and innervation of such naturally occurring hairs. The effort focuses on mimicking the performance of the bio-sensors, not producing an exact replica. The resulting sensor should exhibit an ability to: (1) measure fluid motion (2) detect a wake (3) perform repeatable measurements (4) work in a variety of environments and (5) demonstrate longevity. Through this effort research questions answered include:

- How do spider hairs respond to varying loading conditions?

- What are material properties of pinniped vibrissae?

- How does morphology affect vibrissae response to the free flow field and wakes?

- Is a cantilever haircell sensor adequate for wake tracking?

- What design characteristics may aid in fluid motion sensor development?

- Can a whisker-like biomimetic sensor capture wake characteristics?

- How can an array of sensors capture the same information as that observed by a harbor seal? 


\subsection{Research}

To answer the preceding questions and develop a bio-inspired whisker-like sensor, this research begins with an investigation of the kinematics of trichobothria on Cupiennius salei. A biologically matched viscoelastic model of the hair is developed. The research progresses to investigating vibrissae found in pinnipeds. Material properties are measured and the whiskers' responses to unobstructed and obstructed flows are observed.

To investigate the ability of a small (under $600 \mu \mathrm{m}$ ) neuromast-like cantilever haircell sensor's performance, a finite element code (FEA) is developed to predict the sensor response to prescribed fluid flows. This model incorporates fluid structure interaction to include the forces relative to the drag and added mass.

The prior research leads to the development of a large (over $2 \mathrm{~cm}$ ) whisker-like sensor inspired by seal vibrissae. Several revisions are created and each is tested, modeled, and discussed. The final revision is packaged into an 8 sensor array designed to for minimal obstruction to the fluid flow, capable of carrying on board electronics, and being carried by a harbor seal while tracking a RC submarine.

This dissertation is organized as follows. Chapter 2 reviews the relevant background literature on wake hydrodynamics, hair and whisker sensory systems found in nature, traditional flow sensing measurement techniques, and current biologically inspired sensors. Chapter 3 presents a kinematic model of spider trichobothria. Chapter 4 describes the experimental investigation of pinniped vibrissae developing material properties and quantifying the whiskers' response to fluid motion. Chapter 5 develops a FEA code to predict a haircell inspired sensor's response to prescribed fluid motion and illustrates how different parameters affect the sensor motion. Chapter 6 illustrates the design and development of a whisker-like sensor for detecting and tracking hydrodynamic signals. Chapter 7 explains the design of packaging the sensor into an array for field trials and presents some preliminary data of the sensor array monitoring the fluid signals tracked by a harbor seal while chasing a submarine. Chapter 8 
summarizes the findings and conclusions of the research and discusses future research directions and aims. 


\section{Chapter 2}

\section{Background}

\subsection{Wake Hydrodynamics}

Both inanimate and animate objects moving under water at characteristic Reynolds numbers larger than unity generate wakes with specific hydrodynamic characteristics associated with the field motions of the fluid. The mean flow quantities and spectral characteristics of these wakes differ depending on the mechanisms producing the motions of the objects, which are coupled to their geometries. Due to the focus on seal vibrissae tracking of hydrodynamic signals, a review of fish wakes is first presented indicating information potentially available to the seal.

The wake of a swimming fish generates a complex flow field containing vortical structures [27 33]. A range of wake structures were observed based on fish body length, body motion, and swimming speed [31]. The vortical structures present in the wakes varied from the simple vortex structure of a goldfish [29] to vortex rings in behind rainbow trout [28] and chub mackerel [32]. The wake structure lasted long after the fish had passed. Hanke and Bleckmann showed through particle image velocimetry measurements that the water velocities decayed rapidly just after the fish passed but nevertheless remained measurable for up to 5 minutes afterwards [27]. A goldfish wake showed vortical structure 30 seconds after swimming by the 
measurement point and residual velocities remained for 3 minutes. The wake behind the goldfish was nearly three times as wide as the body length [29]. The sense of rotation of these vortices caused by the caudal fin is opposite that of a classical Karman vortex street [34].

Due to the vortical structure residing in the hydrodynamic trail of fish locomotion, cylinder wakes are used as test cases in Chapters 4, 6, and 7. The experiments conducted in this dissertation involve Reynolds numbers between 100 and 2500. The Karman vortex street and shedding frequencies in this range are well documented [35-41].

\subsection{Biological Hair and Whisker Sensors}

Several biological sensory systems are reviewed for inspiration in sensor design and to illustrate the high sensitivities of these systems found in nature.

\subsubsection{Fish}

Fish obtain information about the surrounding fluid environment through a distribution of neuromasts. These include external neuromasts located on the surface of the fish and neuromasts located within sub-epidermal canals on the sides of the fish with the internal neuromasts between pairs of periodic pores leading to the external fluid field [16, 18, 42] (Figure 2.1). The surface sensors are exposed to the often turbulent boundary layer along the sides of the fish, while the sensors in the lateral line canal are exposed to very small flows caused by pressure differences between pores [3].The external neuromasts respond to changes in external flow velocity while the canal neuromasts respond to changes in external flow acceleration[43]. Electrophysical measurements of a goldfish taken from the nerves under the lateral line showed that the cupula can detect low frequency vortices shed by an upstream cylinder [44]. Pohlmann et al demonstrated a catfish is capable of tracking wakes $10 \mathrm{~s}$ old and up 


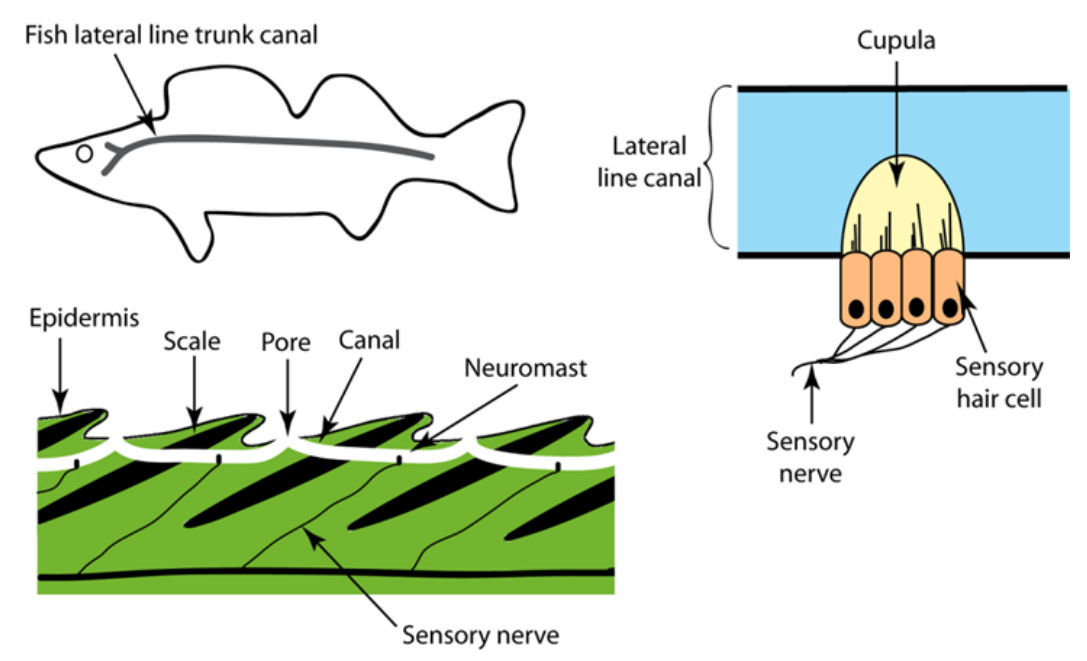

Figure 2.1: Schematic of lateral line canal (left) and a neuromast (right) located between two pores [3].

to 55 body lengths behind the prey [45, 46].

\subsubsection{Spider}

Certain species of spiders such as Cupiennius salei use hairs on their legs called trichobothria to sense air movements around the arachnid (Figure 2.2). Trichobothria are wind-sensing hair-like sensilla on the legs and pedipalps of spiders capable of absorbing the energy of air flowing around them with outstanding efficiency. These hairs are arranged in groups of 2-30 increasing in length towards the leg tip from 100-1400 $\mu \mathrm{m}$. Each individual hair is mechanically tuned to different directions and frequencies ranging from 40-600 $\mathrm{Hz}$. The trichobothria are used by the spider to detect and localize prey that can then be caught in the air even while blinded [4, 23, 47, 48]. The velocities of background flows during the activity period of the spider in its natural habitat are typically smaller than $0.1 \mathrm{~m} / \mathrm{s}$, whereas the flow velocities generated by a buzzing fly can reach values of up to $1 \mathrm{~m} / \mathrm{s}$. This implies the trichobothria do not filter any fluid motion information and typically respond to a sudden change in the air velocity around the spider [49]. 

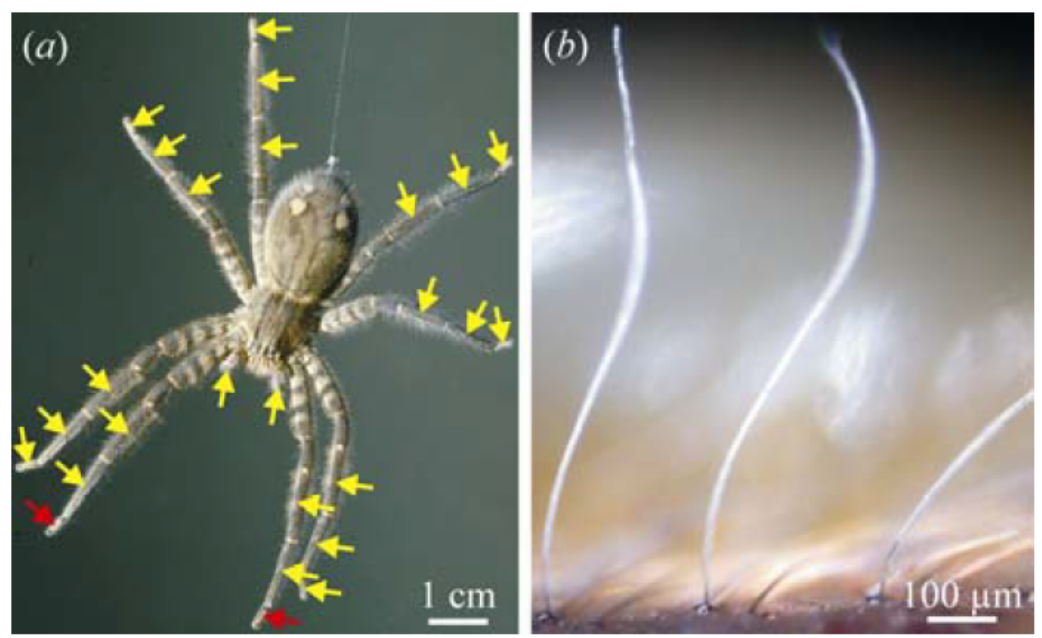

Figure 2.2: (a)Cupiennius salei with trichobothria locations marked with yellow arrows. (b)Close-up of three trichobothria [4].

\subsubsection{Seals}

Vibrissae, or whiskers, are keratinous structures of epidermal origin present in nearly all mammals [50]. Since seals, unlike some marine animals, do not possess an active sonar system [51], the vibrissae are especially well developed in pinnipeds (seals, sea lions, and walrus). The vibrissae are arranged in an array about the face and muzzle and connect to richly innervated follicle sinus complexes below the skin [52 54]. The innervation of aquatic mammals is about 10 times that of land mammals 55 .

In a seminal study Dehnhardt et al used a 'go/no-go' testing paradigm to show that blind-folded and acoustically masked harbor seals are able to discriminate fluid velocities as low as $245 \mu \mathrm{m} / \mathrm{s}$ in the 10-100 Hz range using only their whiskers [26]. In a follow up study, the same group then successfully trained a blind-folded harbor seal to identify and track the wake generated by a propeller-driven miniature submarine again using only its whiskers. The seal was able to successfully locate the submarine in $78.5 \%$ of the trials, even with search onset delays of up to $20 \mathrm{~s}$ [2]. Transitioning the submersible from running to a glide phase caused the harbor seal to lose the sub wake indicating the the rearward thrust flow is more susceptible to seal tracking than a 
passive flow being dragged behind the sub [56]. The vibrissae of harbor seals have also shown the ability to distiguish between object size through haptic touch [57]. More recently, California sea lions have demonstrated similar tracking and touch vibrissae traits [58] and the harbor seal can recognize the direction of the hydrodynamic trail [59]. At certain frequencies, the California sea lion actually has better sensitivity than the harbor seal [60].

Pinniped vibrissae exhibit two surface structures, undulated and smooth. The vibrissae of all Phocidae (true seals), with the exception of the bearded seal (Erignathus barbatus) and monk seal (Monachus spp.), have undulated surfaces. The undulated surface profile is wavy, beaded, or corrugated in appearance and is characterized by repeating crests and troughs along the length of the shaft [50, 157, 61]. In contrast, the vibrissae of all Otariidae (fur seals and sea lions) and Odobenidae (walrus) have smooth surfaces exhibiting none of the wavy surface patterns. Pinnipeds are the only animal group known to possess undulated vibrissae [61].

The unique morphological differences in pinniped vibrissal surface structure have been noted by numerous investigators [50, 57, 62, 63]. Water flow past the whiskers causes vibrations along the vibrissal shaft [64, 65]. Tests holding the broad edge of a ringed seal whisker into a flow caused vibrations up to $300 \mathrm{~Hz}$ while rotating the vibrissae to the narrow edge into the flow eliminated all vibrations [66]. Recent experimental and numerical studies [65, 67, 68] suggest that the undulations serve to minimize vortex shedding behind the whisker, thereby reducing vibrations that would be generated from movement through the water.

\subsection{Biologically Inspired Fluid Motion Sensors}

Using the hairs of a cricket as inspiration, Dijkstra et al. and Krijnen et al. have applied MEMS technology to fabricate arrays of hairs up to $1000 \mu \mathrm{m}$ in length made from SU-8, an epoxy-based polymer. The hairs are supported by membranes 
of two designs leading to different stiffnesses and, therefore, different performance characteristics. The membranes have translational and rotational degrees of freedom normal to the substrate, and the movements of the hairs are detected capacitively via pairs of electrodes built onto the under surfaces of their displaceable membranes and the facing substrate. A loudspeaker-tube combination is used to stimulate and test the sensor array in the ranges $0.1-1.0 \mathrm{~m} / \mathrm{s}$ and $20-100 \mathrm{~Hz}$. The sensors show a peak frequency response at $70 \mathrm{~Hz}$ [69, 70].

Using the neuromasts of the lateral line of fish as inspiration, Fan et al. have also applied MEMS technology followed by magnetic plastic deformation to fabricate individual hair-like motion sensors capable of operating in water. The finished sensor unit consists of a horizontal cantilever beam made of silicon with doped piezoresistor at the base. Attached to the free end of the cantilever is a vertical parallelepipedshaped post made of gold with electroplated Permalloy that is assembled using the magnetic plastic deformation technique. The length, width and thickness of the cantilever beam are $1100 \mathrm{~mm}, 180 \mathrm{~mm}$, and $17 \mathrm{~mm}$, respectively. The length (height), width, and thickness of the post are $820 \mathrm{~mm}, 100 \mathrm{~mm}$, and $10 \mathrm{~mm}$, respectively. The entire unit is coated with a $300 \mathrm{~nm}$ thick Parylene film to provide the necessary electrical insulation for operating in water. Fluid forces acting on the two (length $\mathrm{x}$ width) surfaces of the post create a moment that bends the cantilever beam, and the deflection of the beam is detected by means of piezoresistive strain gauges located at its base. The strain gauges are integrated with the cantilever beam as part of the microfabrication process. Tests with a sensor mounted on a flat plate in laminar flow show that it can detect velocities ranging from approximately 0.2 to $0.9 \mathrm{~m} / \mathrm{s}$ [71]

Using rat vibrissae as inspiration, the Hartmann lab has developed tactile whiskers implementing flex sensors and strain gauges. The sensor presses against a surface causing the whisker to bend. The bending motion is measured via two pairs of strain gauges at the base allowing for a back-end calculation of the location of the force on the whisker. Combing the design into an array, the whiskers can be swept across a 
surface and the resulting bending motions extrapolated to generate a representation of the surface. They showed that their system has potential to detect object distances, determine surface textures, map terrain features, estimate speed, determine wheel slip on a rover, and extract shape parameters of an object [72, 73].

Also using rat vibrissae as inspiration, the Artificial Intelligence Laboratory at the University of Zurich developed tactile sensors by attaching a whisker to the to the diaphragm of an electret microphone. Evaluation of the sensor design was conducted using different whisker materials including actual rat vibrissae super glued to the diaphragm. Rotating the whiskers against different surface roughnesses, frequency information was transfered and recorded from the diaphragm. The natural whiskers cut off at $350 \mathrm{~Hz}$ in frequency response while the stiffer whisker material had a frequency range up to $1400 \mathrm{~Hz}$ [74 77 .

Using seals as inspiration, Hill experimented with a commercially available bendflex sensor and electret sensor. The bend-flex sensor required a minimum flow speed of $0.25 \mathrm{~m} / \mathrm{s}$ to generate a signal. The electret microphone fashioned similar to [74 correctly captured cylinder wake shedding frequencies but with high variability of the amplitude response between tests and matching sensors [78].

Using trichobothria as inspiration, Barbier et al demonstrated the feasibility of using parallel-plate capacitors as a mechanism for sensing deflections of an artificial whisker exposed to a jet of air. For velocities at the nozzle tip of $22 \mathrm{~m} / \mathrm{s}$ at a distance of $6.5 \mathrm{~cm}$ from the exposed whisker, Barbier positively detected the motion and correlated the signal generated with the direction of the incoming flow [9]. 


\section{Chapter 3}

\section{Trichobothria Kinematics}

As discussed in the previous chapter, spiders possess an array of highly sensitive fluid motion sensors. They are capable of detecting air flow rates as small as $1 \mathrm{~mm} / \mathrm{s}$ [5] through hair motions of only 0.01 degrees. The trichobothria themselves do not deflect under these load conditions and only pivot at the base where the nervous system registers the hair rotation. Due to this high sensitivity it is important to understand the kinematics that govern the hair response to a given stimulus. Such information plays a vital role in the development of sensors to monitor fluid motion. The goal of the research conducted here is to present a kinematic model of trichobothria mechanics through viscoelastic modeling. Such effort previously has been unable to capture the range of motion and has relied on mathematical assumptions for input forces and torques. These results provide insight into the spider's keen sensing ability and provide guidance for the development of similarly sized fluid motion hair sensors. This chapter outlines tests conducted on live specimens leading to the development of new viscoelastic parameters governing trichobothria motion. The major contribution of this chapter is developing the kinematic equation of motion to describe trichobothria under all loading conditions, which ties into the thesis statement by determining key design parameters that can aid in the creation of a bio-inspired fluid motion sensor. Research questions answered in this chapter include: 
- What kinematic model describes trichobothria motion?

- What are the parameters describing hair motion?

- Can the model be simplified to easily describe trichobothria motion?

- What features/characteristics may aid in the development of fluid motion sensor?

The author was not present at the deflection testing on the spiders. The work conducted by the author involves the data analysis and kinematic modeling described after the experimental data section. The experimental methodology and data are included here to illustrate how the kinematics were developed.

\subsection{Experimental Methods}

Surface force spectroscopy (SFS) tests were conducted at the Georgia Institute of Technology and outlined in McConney et al [4]. In these tests live female Cupiennius salei were anesthetized and mounted to a Perspex substrate leaving a front leg exposed. Hairs surrounding the trichobothria were removed and the trichobothria were trimmed to lengths of $100 \mu \mathrm{m}$ or less to avoid interference with the atomic force microscope's (AFM) cantilever. Trichobothria length measurements were made with a Leica DM 4000M microscope.

All AFM and SFS measurements were performed using a Multimode, Nanoscope IIIa microscope with a Picoforce module and a Dimension 3000, Nanoscope IIIa microscope. The cantilever used in the measurements was silicon back-side coated with aluminum and silicon nitride tips of nanometer scale radius. The trichobothria were deflected at constant angular velocity perpendicular to the long axis of the hair shaft. The mechanical characteristics of hair motion are nearly isotropic for all directions [79]. 


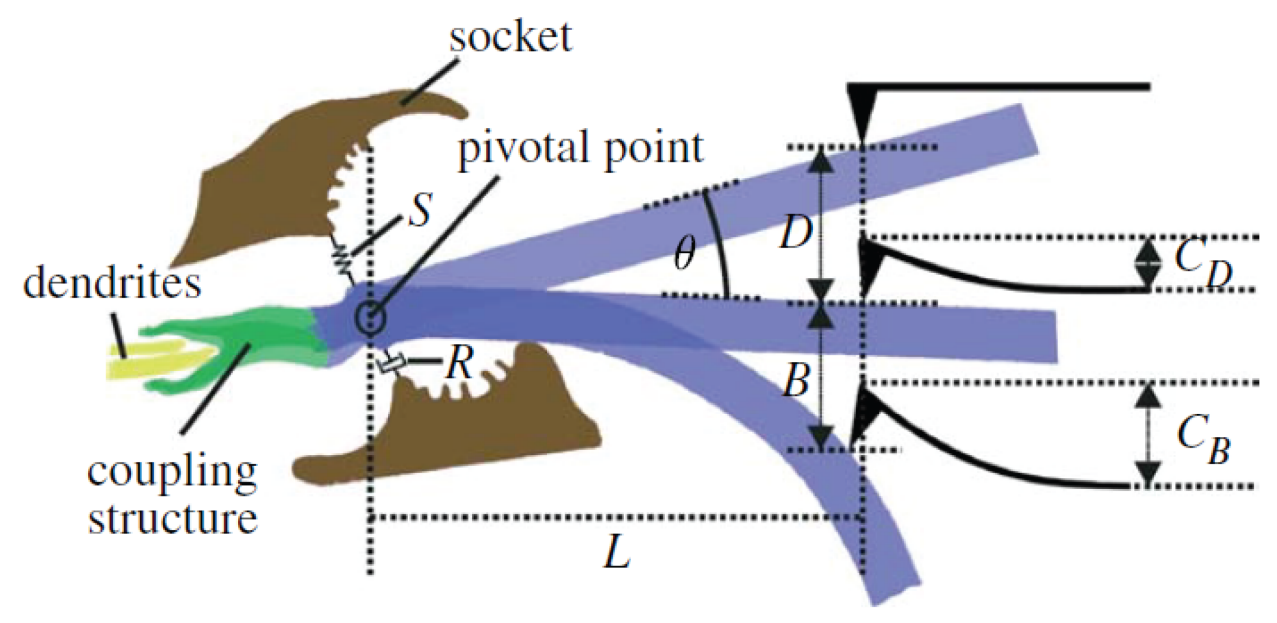

Figure 3.1: Hair parameters. Only the pivoting regime is investigated, region D [4]

Figure 3.1 illustrates the test setup. The AFM cantilever contacts the hair at its natural resting position. It then applies a force at constant angular velocity causing the trichobothria to pivot generating a deflection of $\theta$ occurring in region $\mathrm{D}$. Once the hair strikes the socket it stops pivoting and begins to bend, region B. For an investigation of the kinematics of trichobothria motion only region $\mathrm{D}$ is examined. This is the region of hair motion eliciting a response from the spider to a stimuli.

The morphology of trichobothria presented a unique challenge to point load the hairs. The main hair shaft has many microtrichs branching outward generating a hair on hair morphology (Figure 3.3(a)). Initial loading tests showed instabilities in the force-displacement curves as shown in Figure 3.2 a. This was caused by interactions between the point of the cantilever and the microtrichs.

Repeated loading of the hairs eventually resulted in a significant data improvement shown in Figure 3.2 b. The repetition of the loading effectively microshaved the microtrichs from the main shaft of the trichobothria (Figure 3.3(b)). The slight horizontal displacements of the cantilever tip relative to the hair made the surface smooth and allowed the AFM to properly deflect the trichobothria. The horizontal displacement of the tip during testing altered the point force from the pivot location 

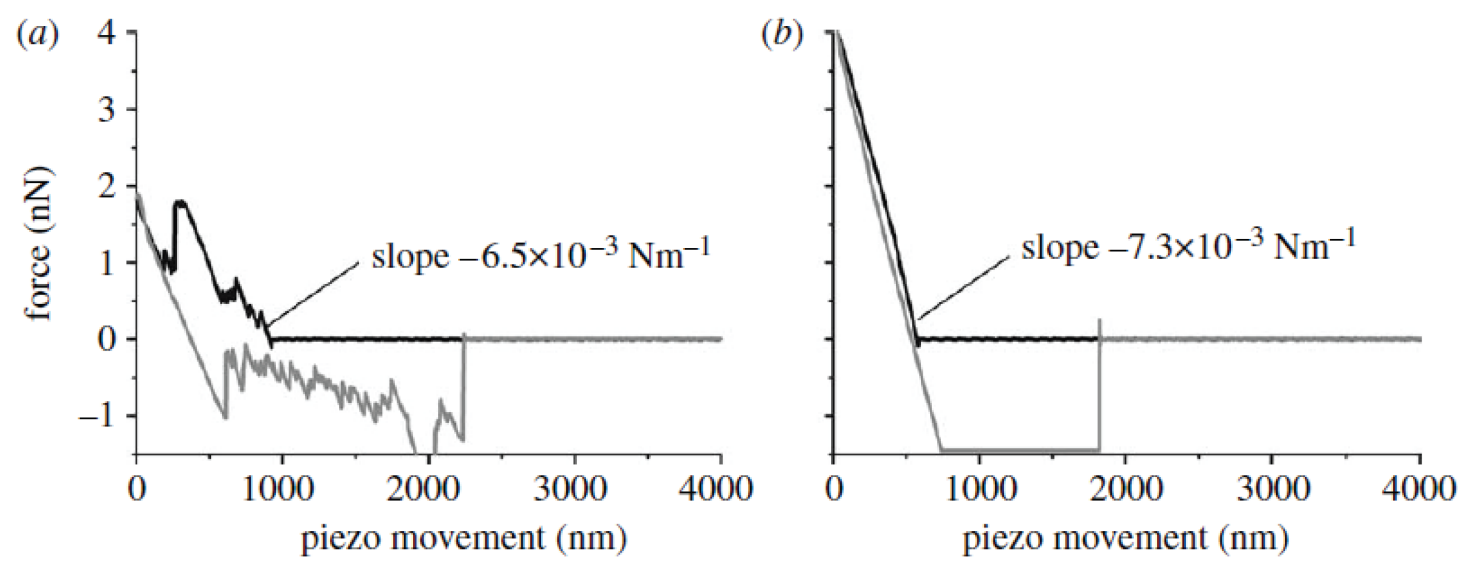

Figure 3.2: Comparison of results (a) before shaving and (b) after [4].

of the hair. However, this distance was very small and did not affect calculations of applied torque.

\subsection{Experimental Results}

The tests assume the hairs do not bend in the pivot regime and the AFM cantilever tip does not penetrate into the hair itself. The no-bending assumption was verified by applying the same force at increasing distances for the pivot point leading to a quadratic increase in hair displacement consistent with a rigid beam. The indentation of the cantilever tip into the hair was less than $1 \mathrm{~nm}$ and was close to the experimental uncertainty [4].

Significant instabilities were observed in the large scale deflections occurring in loads over $100 \mathrm{nN}$. These are attributed to the interactions between the AFM cantilever and hair. Thus tests were conducted under small loading conditions (up to $2 \mathrm{nN}$ ). These resulted in linear hair deflections. Barth and Holler [5] found a trichobothria only need to deflect by a angle of 0.01 degrees to elicit a nervous response from the spider's mechanoreceptors. For the tests presented here this threshold is reached by loads of 0.07 to $0.35 \mathrm{nN}$ depending on the angular velocity (Figures 3.4 and 3.5 a). 

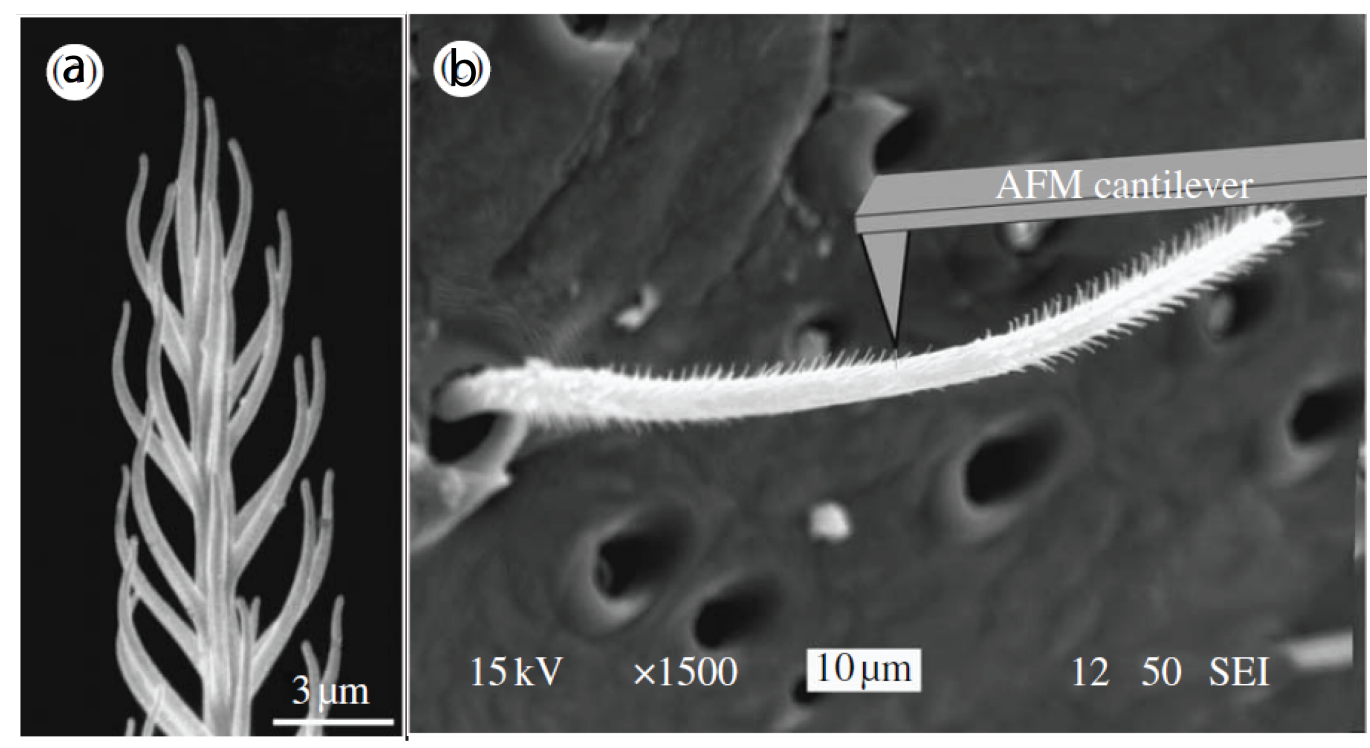

Figure 3.3: (a) SEM micrograph of trichobothria tip showing microtrichs around the main hair shaft. (b) shortened trichobothria with partially removed microtrichs [4].

Under the small loading conditions the trichobothria were found to deflect linearly with force ranging from $12.5 \mathrm{pN}$ to $1.5 \mathrm{nN}$ as shown in Figure 3.4. The hair displaced by $0.85 \mathrm{~nm}$ for every $12.5 \mathrm{pN}$ increase in load. The response remained linear even below the 0.01 angle threshold. The torque resisting hair motion is directly related to the loading curve by multiplying the force applied to the trichobothria by the length of the lever arm.

Applying varying angular velocities ranging from 0.0004 to $0.259 \mathrm{rad} / \mathrm{s}$ resulted in the load-deflection plots shown in Figure 3.5(a). The mechanical resistance to deflection forces drops significantly for angular velocities below $0.05 \mathrm{rad} / \mathrm{s}$. The changing load-displacement curves for varying inputs indicates a viscoelastic response. As the loading rate increases, the results begin to converge, moving toward a more elastic response.

To generate data for the kinematic models 81 tests were run on a single hair of original length $950 \mu \mathrm{m}$. All data points were taken at a pivot angle of $1.23 \times 10^{-3} \mathrm{rad}$. The torque was calculated by multiplying the load to reach the prescribed deflection by the length of the moment arm. Results are shown in Figure 3.6(a). The calculation 


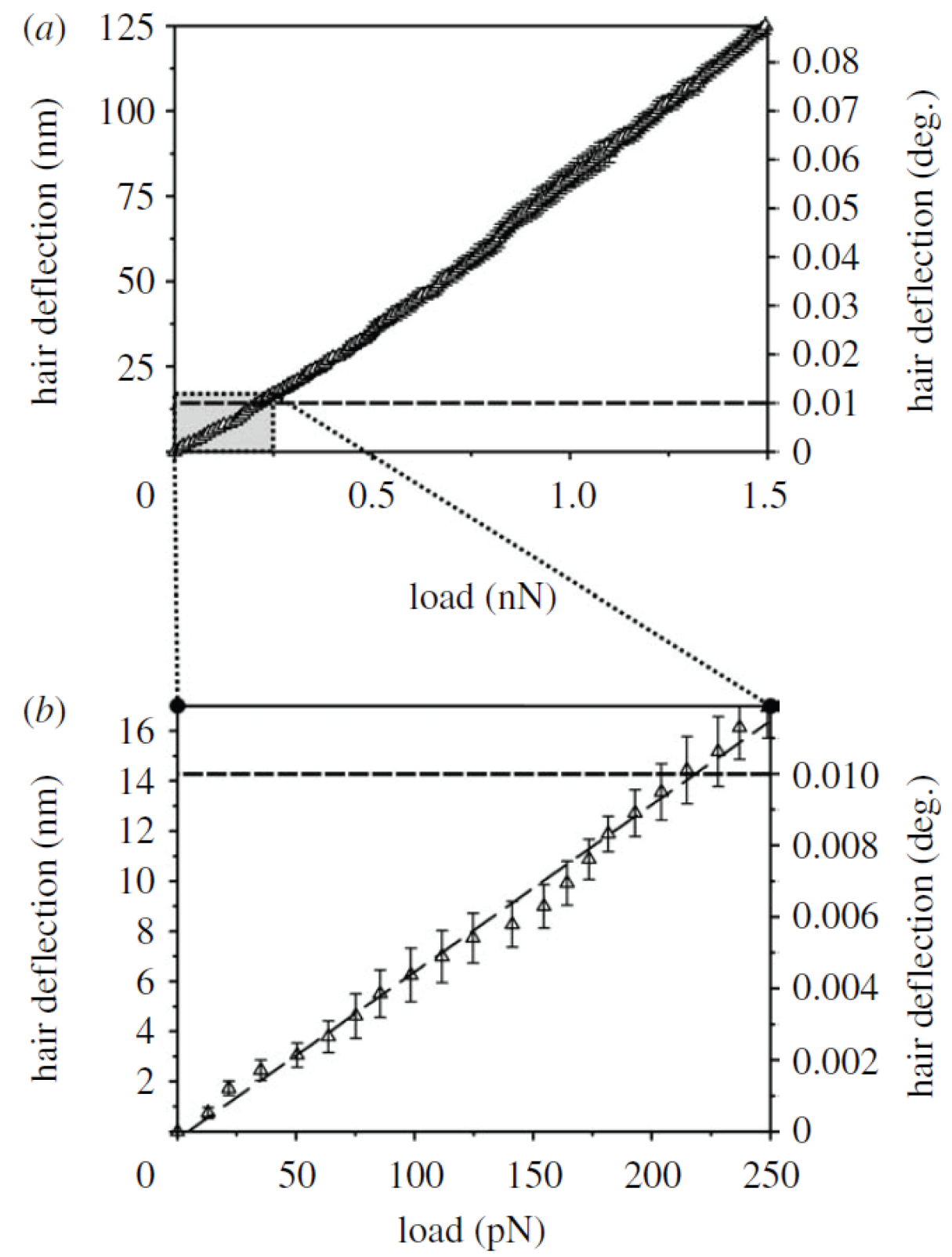

Figure 3.4: $114.5 \mu \mathrm{m}$ Trichobothria deflection measurement obtained at an angular velocity of $0.029 \mathrm{rad} / \mathrm{s}$. (a) Load-deflection curve (b) enlarged initial region. The dashed line represents the minimum angle necessary to elicit a nervous response from the spider [5] [4]. 
(a)

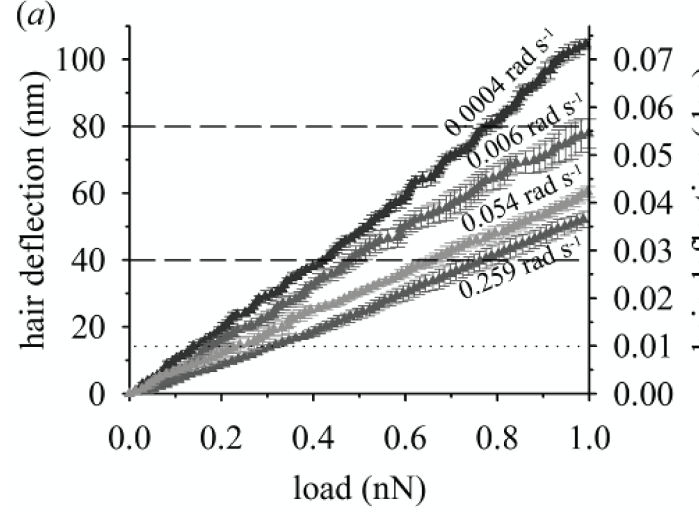

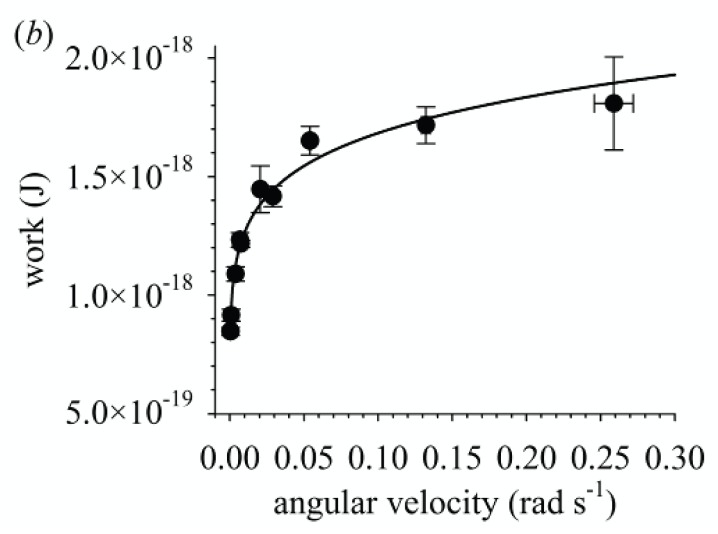

Figure 3.5: Angular velocity dependence of the force-displacement results from 12 tests. (a) Hair pivots more at lower angular velocities. (b) Work required to reach physiological threshold (0.01 deg)

\begin{tabular}{|c|c|c|c|} 
data point & $\dot{\theta}$ mean $(\mathrm{rad} / \mathrm{s})$ & $\mathrm{T}$ mean $(\mathrm{Nm})$ & $\dot{T}$ mean $(\mathrm{Nm} / \mathrm{s})$ \\
\hline 1 & $4.20 \times 10^{-4}$ & $3.14 \times 10^{-14}$ & $1.08 \times 10^{-14}$ \\
2 & $8.17 \times 10^{-4}$ & $3.41 \times 10^{-14}$ & $2.27 \times 10^{-14}$ \\
3 & $3.84 \times 10^{-3}$ & $4.04 \times 10^{-14}$ & $1.27 \times 10^{-13}$ \\
4 & $6.81 \times 10^{-3}$ & $4.59 \times 10^{-14}$ & $2.55 \times 10^{-13}$ \\
5 & $7.37 \times 10^{-3}$ & $4.52 \times 10^{-14}$ & $2.72 \times 10^{-13}$ \\
6 & $2.05 \times 10^{-2}$ & $5.39 \times 10^{-14}$ & $8.97 \times 10^{-13}$ \\
7 & $2.89 \times 10^{-2}$ & $5.27 \times 10^{-14}$ & $1.24 \times 10^{-12}$ \\
8 & $5.39 \times 10^{-2}$ & $6.14 \times 10^{-14}$ & $2.70 \times 10^{-12}$ \\
9 & $1.32 \times 10^{-1}$ & $6.39 \times 10^{-14}$ & $6.88 \times 10^{-12}$ \\
10 & $2.59 \times 10^{-1}$ & $6.73 \times 10^{-14}$ & $1.41 \times 10^{-11}$ \\
\hline
\end{tabular}

Table 3.1: Data used to determine kinematic mechanics of trichobothria. Data points are from 81 trials

of torque rate is described in the 3 parameter modeling section. The complete data set can be found in Table 3.1 .

\subsection{Viscoelastic Models}

Elastic materials have capacity to store mechanical energy without any dissipation of the energy. They are modeled through springs and modulus of elasticity. Newtonian viscous fluids (in a non-hydrostatic stress state) have capacity to dissipate energy, but none to store it. They are modeled through dashpots and viscosity. Viscoelastic 

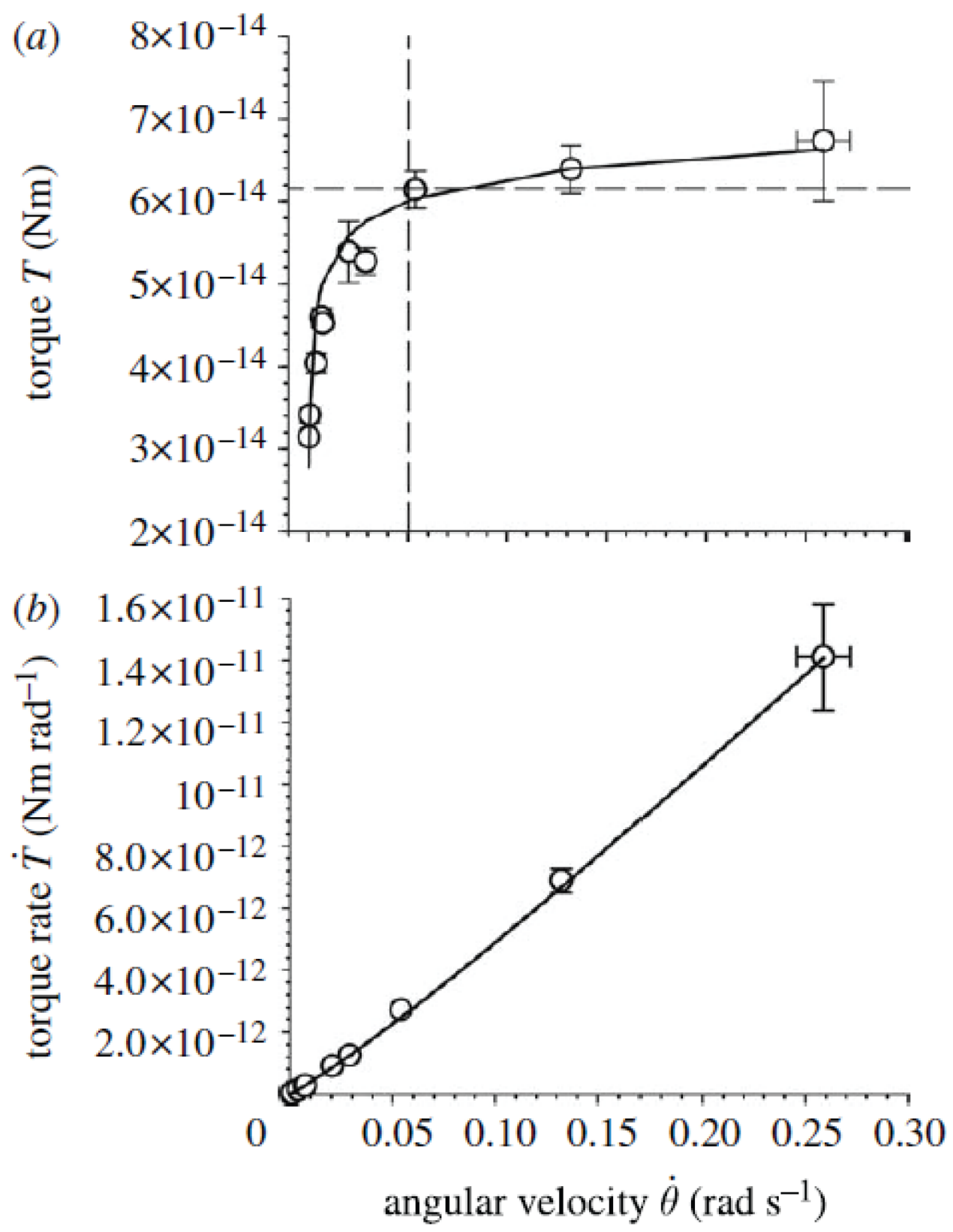

Figure 3.6: Comparison of measurements (circles) and empirical fits of (a) torque (b) the torque time rate of change 


\begin{tabular}{|c|c|c|c|} 
data point & $\dot{\theta} \mathrm{rms}(\mathrm{rad} / \mathrm{s})$ & $\mathrm{T} \mathrm{rms}(\mathrm{Nm})$ & $\dot{T} \mathrm{rms}(\mathrm{Nm} / \mathrm{s})$ \\
\hline 1 & $2.25 \times 10^{-6}$ & $6.09 \times 10^{-16}$ & $3.06 \times 10^{-16}$ \\
2 & $7.43 \times 10^{-6}$ & $9.22 \times 10^{-16}$ & $7.91 \times 10^{-16}$ \\
3 & $4.04 \times 10^{-5}$ & $1.15 \times 10^{-15}$ & $4.61 \times 10^{-15}$ \\
4 & $6.65 \times 10^{-5}$ & $1.12 \times 10^{-15}$ & $8.41 \times 10^{-15}$ \\
5 & $3.78 \times 10^{-5}$ & $5.78 \times 10^{-16}$ & $6.60 \times 10^{-15}$ \\
6 & $6.07 \times 10^{-4}$ & $3.70 \times 10^{-15}$ & $6.94 \times 10^{-14}$ \\
7 & $3.99 \times 10^{-4}$ & $1.64 \times 10^{-15}$ & $4.90 \times 10^{-14}$ \\
8 & $9.40 \times 10^{-4}$ & $2.25 \times 10^{-15}$ & $1.22 \times 10^{-13}$ \\
9 & $2.84 \times 10^{-3}$ & $2.90 \times 10^{-15}$ & $3.72 \times 10^{-13}$ \\
10 & $1.32 \times 10^{-2}$ & $7.30 \times 10^{-15}$ & $1.71 \times 10^{-12}$ \\
\hline
\end{tabular}

Table 3.2: RMS values for data used to determine kinematic mechanics of trichobothria. Data points are used to determine the confidence intervals of the viscoelastic parameters.

materials demonstrate both capabilities and as such are modeled via a combination of springs and dashpots [80]. Thus viscoelastic materials are time dependent and the behavior is expressed by a constitutive equation including time as a function as well as stress and strain.

The angular velocity dependency of the trichobothria response (Figure 3.5) to applied loads indicate the hair is supported by a viscoelastic material. Traditional analysis of the kinematics have relied on a linear two-parameter model to describe the hair motion. Here, viscoelastic kinematics for the hair are described in a torsional reference frame and the equations of motion are developed for a two and three parameter viscoelastic material.

\subsubsection{Two Parameter Kelvin Solid Model}

In this approach, the suspension supporting the hair is approximated as a two parameter Kelvin solid model consisting of a spring and a dashpot element connected in parallel (Figure 3.7). The hair is assumed to behave as a simple forced damped harmonic oscillator for which the conservation of angular momentum is given by Equation 3.1. The kinematics of trichobothria motion of previous studies used this model to predict hair motion in response to given air motions. 


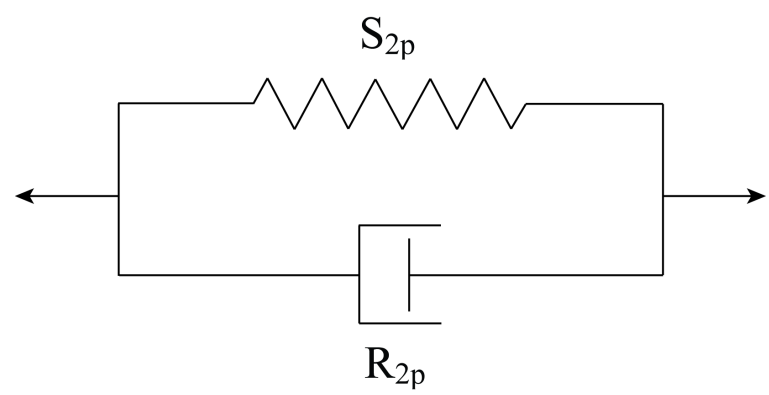

Figure 3.7: Spring and dashpot representation of the two parameter model.

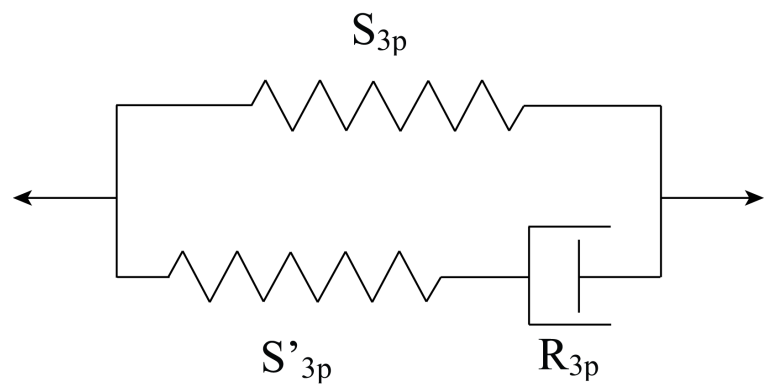

Figure 3.8: Spring and dashpot representation of the three parameter model

$$
I \ddot{\theta}+R_{2 p} \dot{\theta}+S_{2 p} \theta=T
$$

Here I $\left(\mathrm{Nm} s^{2} / \mathrm{rad}\right)$ is the trichobothria's moment of inertia; $R_{2 p}(\mathrm{Nm} \mathrm{s} / \mathrm{rad})$ is the hair's damping constant; $S_{2 p}(\mathrm{Nm} / \mathrm{rad})$ is the torsional restoring constant; T $(\mathrm{Nm})$ is the torque applied to the hair; and $\theta(\mathrm{rad}), \dot{\theta}(\mathrm{rad} / \mathrm{s})$, and $\ddot{\theta}\left(\mathrm{rad} / \mathrm{s}^{2}\right)$ are the deflection angle, velocity, and acceleration respectively.

Each experiment occurred at a constant angular velocity $\dot{\theta}$. The torque associated with air friction and air added mass are deemed negligible compared with the torque imparted by the cantilever probe, $T_{S F S}$. This reduces Equation 3.1 to Equation 3.2 .

$$
R_{2 p} \dot{\theta}+S_{2 p} \theta=T_{S F S}
$$




\subsubsection{Three Parameter Solid Model}

In this approach the suspension supporting the hair is modeled as a three parameter viscoelastic system consisting of a spring element in parallel with a spring and dashpot in series as shown in Figure 3.8. This type of viscoelastic model has been previously documented by Flugge [81], Fung [82, and Fuchigami [83] but never applied to trichobothria sensors due to limitations of experimental data and complexities of the hair motion. The corresponding angular momentum equation is given by Equation 3.3.

$$
I \ddot{\theta}+R_{3 p}\left(1+\frac{S_{3 p}}{S_{3 p}^{\prime}}\right) \dot{\theta}+S_{3 p} \theta=T+\frac{R_{3 p}}{S_{3 p}^{\prime}} \dot{T}
$$

Here the torsional constants are $S_{3 p}$ and $S_{3 p}^{\prime}$. The damping constant is represented by $R_{3 p}$. The quantity $\dot{T}$ is the time rate of change of the torque. It is estimated form the relation $\dot{T} \cong T_{t o t} \dot{\theta} / \theta_{\text {tot }}$ where $\theta_{\text {tot }}$ is the total angular deflection of the trichobothria during testing. As previously mentioned $\dot{\theta}$ is fixed and $T_{t o t}=T_{S F S}$. Therefore Equation 3.3 simplifies to Equation 3.4 .

$$
R_{3 p}\left(1+\frac{S_{3 p}}{S_{3 p}^{\prime}}\right) \dot{\theta}+S_{3 p} \theta=T_{S F S}+\frac{R_{3 p}}{S_{3 p}^{\prime}} \dot{T_{S F S}}
$$

In these models the hair is modeled as a straight cylinder allowing the moment of inertia to be describe by Equation 3.5 .

$$
I=\frac{\pi \rho_{h} d^{2}}{48}\left(4 L^{3}+\frac{3}{4} d^{2} L\right)
$$

where d, L, and $\rho_{h}$ are the diameter, length, and density of the trichobothria.

\subsection{Multiple Regression}

The viscoelastic parameters were found via a nonlinear multiple regression analysis outlined here. In a multiple regression analysis the data must consist of $\mathrm{n}$ values (data 
points) for each of the $\mathrm{m}$ independent variables $\left(X_{i j}\right)$ and $\mathrm{n}$ values of the dependent variable $y_{i}$. There are $\mathrm{m}$ variables to be determined $\left(\beta_{j}\right)$. The error $\left(\epsilon_{i}\right)$ is assumed normal with a mean of zero [84].

$$
y_{i}=\sum_{j=1}^{m} X_{i j} \beta_{j}+\epsilon_{i}
$$

Using the three parameter solid model as an example, torque became the dependent variable. Torque rate, angle of deflection, and angular velocity became the independent variables. The kinematic equation of motion, Equation 3.4 was rewritten as:

$$
T=-\frac{R_{3 p}}{S_{3 p}^{\prime}} \dot{T}+S_{3 p} \theta+\left(R_{3 p}+\frac{R_{3 p} S_{3 p}}{S_{3 p}^{\prime}}\right) \dot{\theta}
$$

The parameters to be solved are now:

$$
\begin{gathered}
\beta_{1}=\frac{R_{3 p}}{S_{3 p}^{\prime}} \\
\beta_{2}=S_{3 p} \\
\beta_{3}=R_{3 p}+\frac{R_{3 p} S_{3 p}}{S_{3 p}^{\prime}}
\end{gathered}
$$

The parameters of interest are found by solving the normal equations:

$$
\sum_{i=1}^{m} \sum_{k=1}^{n} X_{i j} X_{i k} \beta_{k}=\sum_{i=1}^{m} X_{i j} y_{i}
$$

In matrix notation this becomes:

$$
\left(X^{T} X\right) \beta=X^{T} y
$$

And the variables are thus solved for by:

$$
\beta=\left(X^{T} X\right)^{-1} X^{T} y
$$


The residuals, $r_{i}$, are the difference between the observed values and those calculated by the model:

$$
r_{i}=X_{i}-X_{i}^{c a l c}
$$

The goal of the regression is to minimize the sum of the residuals, S, given by:

$$
S=\sum_{i=1}^{m} r_{i}^{2}
$$

The variance $\left(\hat{\sigma}^{2}\right)$ is determined by:

$$
\hat{\sigma}^{2}=\frac{S}{n-m}
$$

With standard error:

$$
\hat{\sigma}_{j}^{2}=\sqrt{\left.\hat{\sigma}\left[X^{T} X\right)^{-1}\right]_{j j}}
$$

The confidence interval, $\mathrm{C}$, is found via:

$$
C= \pm t_{\frac{\alpha}{2}, n-m} \hat{\sigma}_{j}^{2}
$$

$t_{\frac{\alpha}{2}, n-m}$ is the value from a two sided $\mathrm{t}$ distribution. For the data examined here $n=10$ (number of data sets) and $m=3$ (number of independent variables).

It is straightforward to then resolve the solutions for $\beta$ into the kinematic parameters of interest $R_{3 p}, S_{3 p}$, and $S_{3 p}^{\prime}$. The confidence interval solutions are for the $\beta$ terms. A description of how the propagation of the error in the confidence intervals translates to the kinematic parameters is found in the Propagation of Error appendix. 


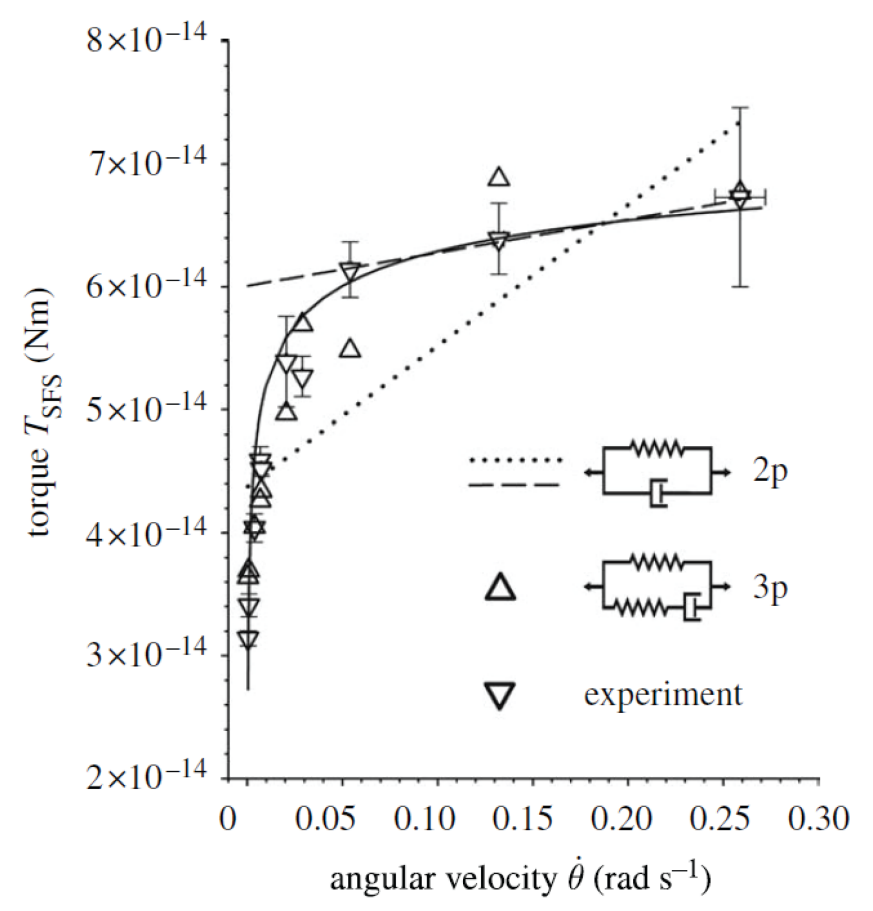

Figure 3.9: Comparison of experimental data and predicted torques from the viscoelastic models

\subsection{Results}

Using the Kelvin model described previously and a multiple regression across all data points the model parameters were found to be $S_{2 p}=3.57 \times 10^{-11}\left( \pm 8.08 \times 10^{-12}\right)$ $\mathrm{Nm} / \mathrm{rad}$ and $R_{2 p}=1.15 \times 10^{-13}\left( \pm 1.05 \times 10^{-13}\right) \mathrm{Nm} \mathrm{s} / \mathrm{rad}$. This model can be checked by calculating the torque from Equation 3.1 for the values of $\dot{\theta}$ in Table 3.1 and the values obtained for $S_{2 p}$ and $R_{2 p}$. This results in the dotted line in Figure 3.9. The difference between the measurements and the calculations averaged over the entire range of angular velocities is $14 \%$, reaching values as high as $39 \%$ at the smallest angular velocity. Owing to the poor agreement, especially at low angular velocities, a second regression was performed using only the data corresponding to $\dot{\theta} \geq 0.05$ $\mathrm{rad} / \mathrm{s}$. From this, it was found that $S_{2 p}=4.89 \times 10^{-11}\left( \pm 2.88 \times 10^{-13}\right) \mathrm{Nm} / \mathrm{rad}$ and $R_{2 p}=2.83 \times 10^{-14}\left( \pm 2.07 \times 10^{-15}\right) \mathrm{Nm} \mathrm{s} / \mathrm{rad}$, which yielded calculated values of $T_{S F S}$ differing from those measured by less than $0.5 \%$ (dashed line in Figure 3.9).

For the three-parameter solid model described, the multiple linear regression of 
the measured data in table 2 using Equation 3.4 yielded $S_{3 p}=2.91 \times 10^{-11}( \pm 6.55 \times$ $\left.10^{-12}\right) \mathrm{Nm} / \mathrm{rad}, S_{3 p}^{\prime}=2.77 \times 10^{-11}\left( \pm 9.84 \times 10^{-12}\right) \mathrm{Nm} / \mathrm{rad}$, and $R_{3 p}=1.46 \times 10^{-12}$ $\left( \pm 9.26 \times 10^{-13}\right) \mathrm{Nm} \mathrm{s} / \mathrm{rad}$. The results of the three parameter model using the experimentally determined data of angular velocity and torque rate are plotted as the upward pointing triangle in Figure 3.9. The three-parameter model correctly captures the physical behavior of the hair over the entire range of angular velocities including the drop of torque $\mathrm{T}$ for the small velocities, with an average absolute difference of $7 \%$, the largest difference being $16 \%$ at the smallest angular velocity.

\subsection{Discussion}

The three-parameter solid model for the viscoelastic hair suspension is shown to apply over the entire range of angular velocities explored $\left(4 \times 10^{-4} \leq \dot{\theta} \leq 2.6 \times 10^{-1}\right.$ $\mathrm{rad} / \mathrm{s})$. The other, based on a two-parameter Kelvin solid model, is shown to predict the motion of a hair with much better accuracy for angular velocities $\dot{\theta} \geq 0.05 \mathrm{rad} / \mathrm{s}$, which is in the most biologically relevant range.

Parameter values of the Kelvin model presented here agree within an order of magnitude to those previously determined through mathematical modeling of hair responses to a prescribed flow [48, 85, 86]. Likewise the values of the spring elements in the three parameter model presented here are comparable to previous values. However, the dashpot element is two orders of magnitude larger than previous studies. The three parameter model correctly captures the hair kinematics at low angular velocities which were previously ignored in other studies.

The torque $\mathrm{T}$ tends towards an asymptotic value of $8.50 \times 10^{-14} \mathrm{Nm}$ for very large angular velocities. This is to be expected since the viscoelastic dashpot element resists deformation induced by motions at high angular velocities and, as a consequence, deformation can only occur owing to the spring elements. The latter, however, deform in proportion to the torque irrespective of the rate of change and are responsible for 
the asymptotic behavior observed. This leads to the large errors of predicted hair response over the full data set. The suddenly imposed torque is initially carried entirely by the viscous element (dashpot) thus preventing immediate deflection of the trichobothria. The three parameter model is better suited to the application of high torques as deformation can result via the spring elements for any rate of applied torque.

Focus in sensor design should look for inspiration to the kelvin model matching the high angular velocities. Although much is lost at the lower range, the higher velocities reside in the area of relevance to this research. The sensor under development is designed for aquatic use where the forces acting on the sensor will be higher than those observed in air. The spring parameter of this model is three orders of magnitude greater than that of the dashpot. Thus giving the model the almost purely elastic response observed by the linearity of the model prediction. A sensor designed as a pivoting whisker is potentially accurately designed with reliance of the spring parameter and modeled with an elastic response.

\subsection{Summary}

The goal of this chapter was to model trichobothria motion to capture the hair sensor's response under any loading conditions or stimulus. Reviewing the research questions presented at the start of the chapter, the three-parameter viscoelastic solid

model captured the trichobothria response under all loading conditions for the full range of motion. This model incorporates a spring element in parallel with a spring and dashpot in series with values $2.91 \times 10^{-11}\left( \pm 6.55 \times 10^{-12}\right) \mathrm{Nm} / \mathrm{rad}, 2.77 \times 10^{-11}$ $\left( \pm 9.84 \times 10^{-12}\right) \mathrm{Nm} / \mathrm{rad}$, and $1.46 \times 10^{-12}\left( \pm 9.26 \times 10^{-13}\right) \mathrm{Nm}$ s/rad, respectively. At the higher applied angular displacement rates, the model was accurately simplified to the two parameter Kelvin solid model. More importantly this model was dominated by the spring constant making it nearly elastic in response. The trichobothria show 
that a rigid hair supported by a membrane acting elastically at higher loads is capable of capturing fluid motion. 


\section{Chapter 4}

\section{Vibrissae Study}

As discussed in Chapter 2 the vibrissae of pinnipeds are highly sensitive hairs capable of detecting small hydrodynamic signals and tracking underwater objects as well as tactile object discrimination. The animals possess motion control of their vibrissae array allowing it to adjust flat against the face or push outward in an erect position nearly perpendicular of the muzzle. Dehnhardt et al [2] noted harbor seals firmly hold the whisker array in this erect configuration when tracking hydrodynamic signals. Figure 4.1 shows the erect vibrissae orientations for a harbor seal and California sea lion. This makes pinniped sensors an ideal candidate to examine further for the development of bio-inspired sensors for wake monitoring. The major contribution of this chapter is studying the material properties and response to flow fields of pinniped vibrissae, which ties into the thesis statement by determining key design parameters that can aid in the creation of a bio-inspired fluid motion sensor. Research questions answered in this chapter include:

- What are the material properties of pinniped vibrissae?

- What are the vibrational characteristics of pinniped vibrissae?

- How does vibrissae morphology affect the whiskers' response to free stream flows? 


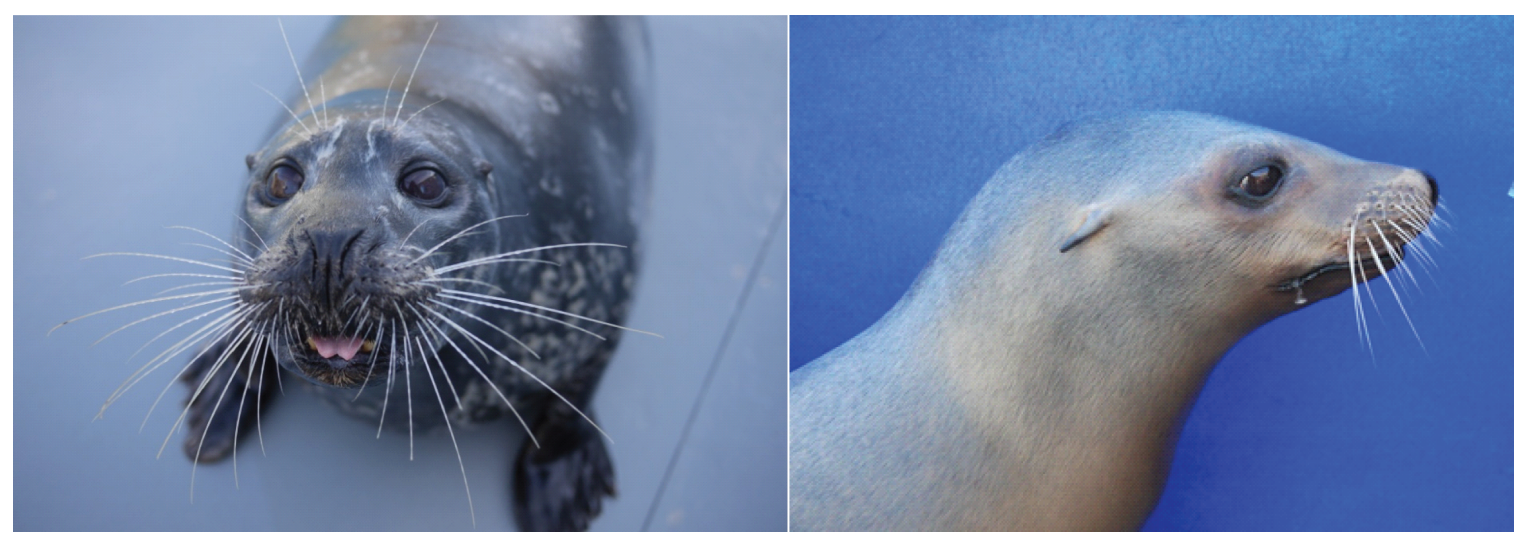

Figure 4.1: Harbor seal (left) and California sea lion (right) vibrissae in erect position.

- How does morphology affect pinniped response to hydrodynamic stimulus?

Unlike the spider trichobothria which monitor fluid motion close to the stationary leg surface, pinniped vibrissae extend outward away from the animal body to monitor the fluid motion outside of boundary layer effects. For the development of a fluid motion sensor likely to be placed on a moving body such as an AUV, vibrissae that are better suited to negate boundary layer effects will provide a basis for sensor design. The pinniped vibrissae also utilized two distinct surface morphologies as discussed in Chapter 2. Although preliminary work has investigated the effects of the surface in vibrissae response, the experimental body of work is very limited. Also lacking in the literature is an evaluation of the actual material properties of pinniped vibrissae. The research presented in this chapter aims to resolve these missing data points through studies conducted on the vibrissae of harbor seals (Phoca vitulina), elephant seals (Mirounga angustirostris), and California sea lions (Zalophus californianus).

The specimens are mounted and subjected to point force deflections and free vibrations to determine basic material properties. Much is still unknown about the effect of vibrissae morphology in actual flows. Here work to resolve these questions is conducted by placing the mounted vibrissae in a flow tank at various orientations and under differing flow conditions to observe the whisker response. Specimen preparation and free flow studies were conducted with the aid of Christin Murphy (University of 


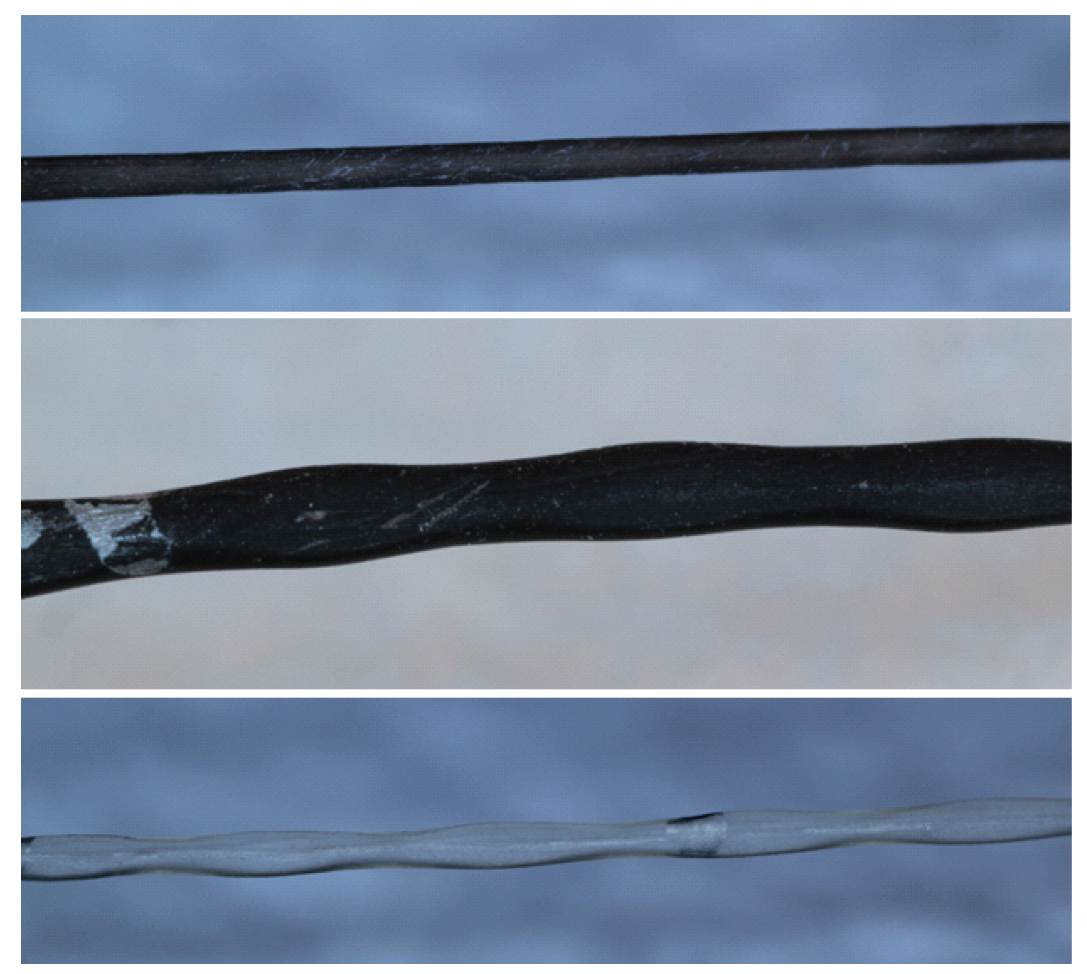

Figure 4.2: Closeup images of smooth vibrissae of California sea lion (top) and undulated vibrissae of elephant seal (middle) and harbor seal (bottom).

South Florida). The use of marine mammal samples was authorized via a letter of authorization to Christin Murphy from the National Marine Fisheries Service.

\subsection{Pinniped Vibrissae}

As previously mention in Chapter 2, many pinniped species possess vibrissae exhibiting unique morphology. Figure 4.2 shows the differences in whisker shape for the species investigated here. The top image shows the smooth surface of the California sea lion. The middle image of an elephant seal whisker and the bottom of a harbor seal exhibit the undulatory structure only found in pinnipeds [61]. The surface is wavy or corrugated characterized by repetition of crests and troughs present over the entire whisker length [87] [88]. The vibrissae of all three species are flattened, giving them an oval cross section, pointing in the direction of fluid motion or the equivalent of the animal swimming/facing forward. 


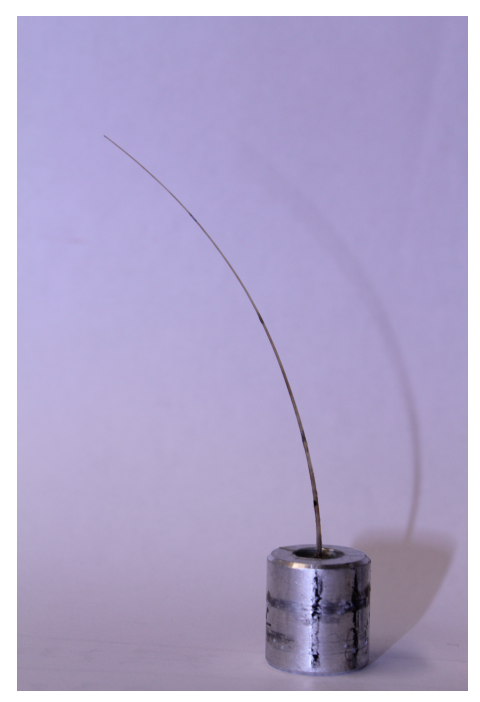

Figure 4.3: California sea lion vibrissae mounted for testing.

\subsection{Specimen Preparation}

Vibrissae specimens from the three pinniped species were collected from postmortem juvenile animals due to the higher mortality rate of younger pinnipeds. A single vibrissae from each animal was excised from the right side of the muzzle. Total, vibrissae were obtained from 9 harbor seals, 8 elephant seals, and 9 California sea lions. The vibrissae were clipped at the base and chosen for matching lengths. The mean length across all samples was $7.7 \mathrm{~cm} \pm 0.55 \mathrm{~cm}$. Test samples were chosen as those closest to the sample mean. Of the samples tested the elephant seal vibrissae were the longest with and average length of $8.19 \mathrm{~cm}$ and harbor seals the shortest with a mean length of $7.35 \mathrm{~cm}$. The full sample is shown in Table 4.1 .

Vibrissae samples were mounted onto cylindrical aluminum bores, Figure 4.3 . The base of each whisker resided $1 \mathrm{~cm}$ below the top of the mount and was secured with epoxy resin. The base of the mount was tapped to 3/8-16 threads for secure attachment to the testing apparatus. The vibrissae were noted to change shape while resting in water. This is attributed to the whiskers dehydrating during transport and preparation. Before testing, the specimen was submerged in fresh water for one hour to rehydrate the whisker and restore it to its natural form. 


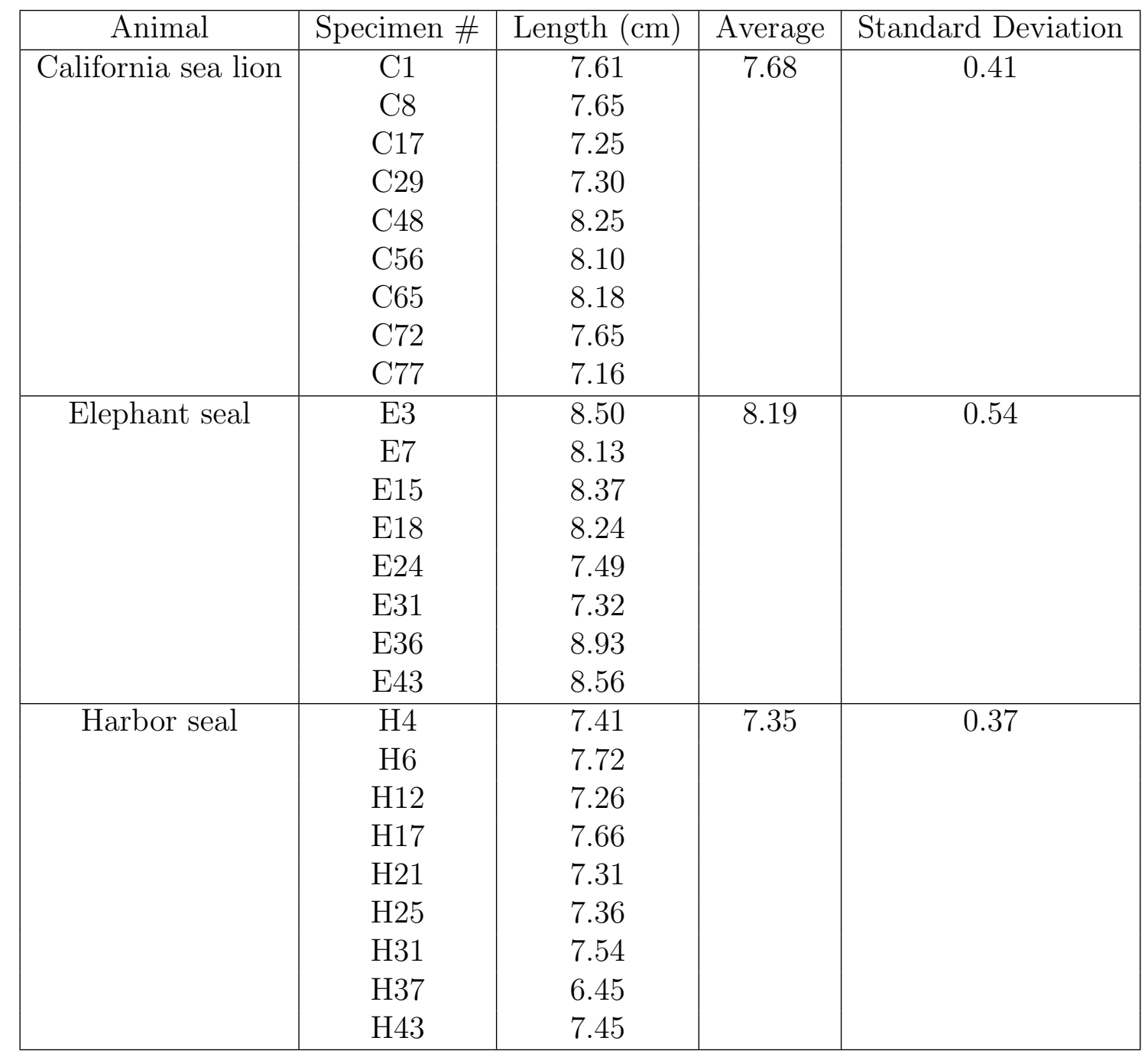

Table 4.1: Vibrissae length measurements 


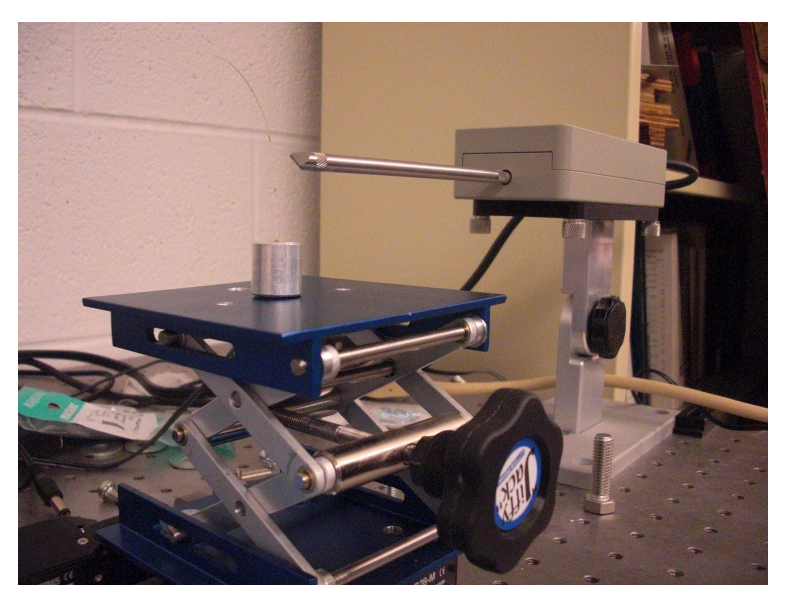

Figure 4.4: Harbor seal whisker mounted for a force-displacement test.

\subsection{Experimental Methods}

\subsubsection{Material Testing}

Force deflection measurements were conducted using a force rig capable of micron level displacements of the vibrissae with millinewton resolution. Motion was provided by a Zaber Technologies model T-NA08A25 linear actuator mounted on an optical table. The actuator had a step size of $0.0476 \mu \mathrm{m}$, travel range of $25.4 \mathrm{~mm}$, maximum speed of $8 \mathrm{~mm} / \mathrm{s}$, and repeatability of under $1 \mu \mathrm{m}$. A jack was attached to the linear actuator to provide height adjustment allowing each measurement to be taken at 2 $\mathrm{cm}$ from the whisker base. The specimens were threaded onto the top of the jack at a 90 degree orientation to the data measurement equipment such that forces were applied directly to the thickest axis. Attempts to take measurements against the thin axis resulted in whisker twisting and dislocations in the data due to the undulations. Point forces on the hair were measured by a Mark 10 model BG012 force gauge. The force gauge had a maximum range of $0.5 \mathrm{~N}$ in $0.0002 \mathrm{~N}$ increments with an accuracy of $\pm 2 \%$ full scale and sampling rate $65 \mathrm{~Hz}$ [89]. In this manner specific whisker deflections at prescribed rates were applied to the midpoint of the specimens. The test rig is shown in Figure 4.4. A similar test design with the same equipment was previously validated for accuracy in beam force displacement by Stocking [11]. 
Free vibration measurements were made under the same conditions as the force deflection measurements with a laser vibrometer replacing the force gauge (and the linear actuator remaining stationary). All vibration measurements were made with a Polytec PDV-100 laser doppler vibrometer measuring velocity. The PDV-100 is a non-contact point-and-shoot portable vibrometer using a helium neon laser to directly measure surface vibrations. The PDV-100 has a measurement range up to $500 \mathrm{~mm} / \mathrm{s}$ with a resolution under $0.2 \mu \mathrm{m} / \mathrm{sHz}^{.5}$ and measurement spot size of $30 \mu \mathrm{m}$ [90]. Using the doppler effect, the vibrometer generates a high frequency carrier signal that experiences a small frequency shift when striking a moving object. The resulting phase modulation is read by the PDV-100 to calculate to speed of the surface motion. The laser was oriented perpendicularly to the thin side of the vibrissae. Due to the elongated cross section the vibrissae had a distinct preference to motion in this direction. Measurements were made at the midpoint of the mounted whisker. The midpoint was marked with silver ink to increase the reflectiveness at the monitor location. The ink had no bearing on the whisker structure or properties. Vibration measurements consisted of 3.2 second samples recorded with Vibsoft version 4.6 from Polytec. Data was collected at $600 \mathrm{~Hz}$ with a resolution of $312.5 \mathrm{mHz}$.

\subsubsection{Flume Study}

Vibration studies of the vibrissae in free stream flows and obstructed flows were conducted in a Rolling Hills Research Company Model 1520 water flume pictured in Figure 4.5. The flume has a test section of $152 \mathrm{~cm}$ length, $38 \mathrm{~cm}$, width, and $46 \mathrm{~cm}$ depth. Flows in the test section range from 0 to $90 \mathrm{~cm} / \mathrm{s}$ with velocity uniformity outside the wall boundary layer of $\pm 2 \%$. Calibration of the water flume is found in the Flume Velocity Field appendix. For testing, individual whiskers on the aluminum mounts were attached via the base threads to a sting arranging the vibrissae in a vertical position. The sting was located in the center of the water column and consisted a stainless steel rod with a 90 degree bend (Figure 4.6). The bend allowed the sting 


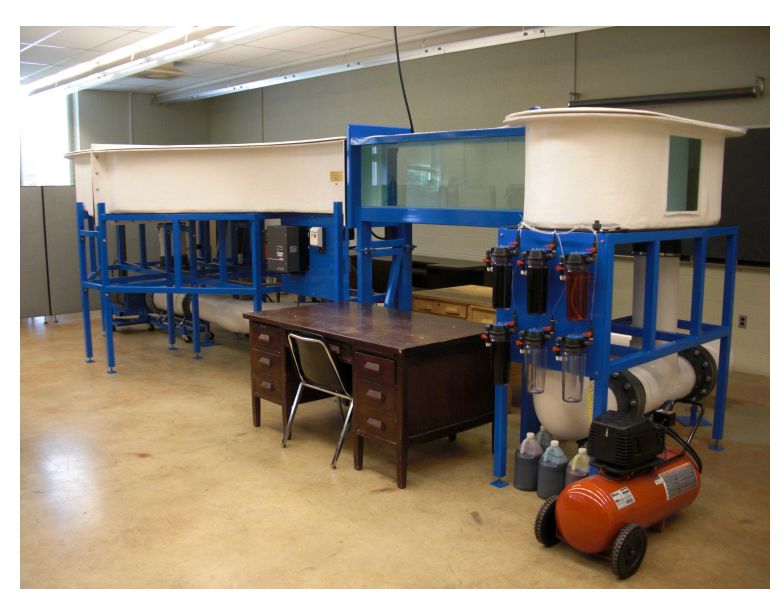

Figure 4.5: Rolling Hills water flume used to test vibrissae response to varying flow conditions.

to reside beneath and downstream of the vibrissae thus having no effect on the fluid motion striking the specimen.

All vibration measurements were made with a Polytec PDV-100 laser doppler vibrometer measuring velocity. The laser was oriented perpendicular to the flow allowing the light to pass directly through the wall of the flume. Test measurements along the length of a vibrissae mounted to the sting in a $0.5 \mathrm{~m} / \mathrm{s}$ flow indicated the vibration was maximal at the whisker tip and proceeded to decrease in magnitude down to the base. Although the tip provided the largest velocities, the hair often moved out of laser line of sight reducing the quality of the recording. The test measurements indicated the frequency of the vibration remained constant along the vibrissae. Thus all measurements were made at the midpoint of the mounted whisker monitoring the vibration of the vibrissae in the direction cross-stream of the flow. Vibration measurements of the sting showed a peak frequency at $14 \mathrm{~Hz}$ with an amplitude under $0.004 \mathrm{~m} / \mathrm{s}$, well below all recordings for the vibrissae. Vibration measurements consisted of 6 second samples recorded with Vibsoft version 4.6 from Polytec.

All free stream tests were conducted at a flow speed of $0.5 \mathrm{~m} / \mathrm{s}$. All vibration measurements were made in the cross-stream direction with the vibrometer laser 


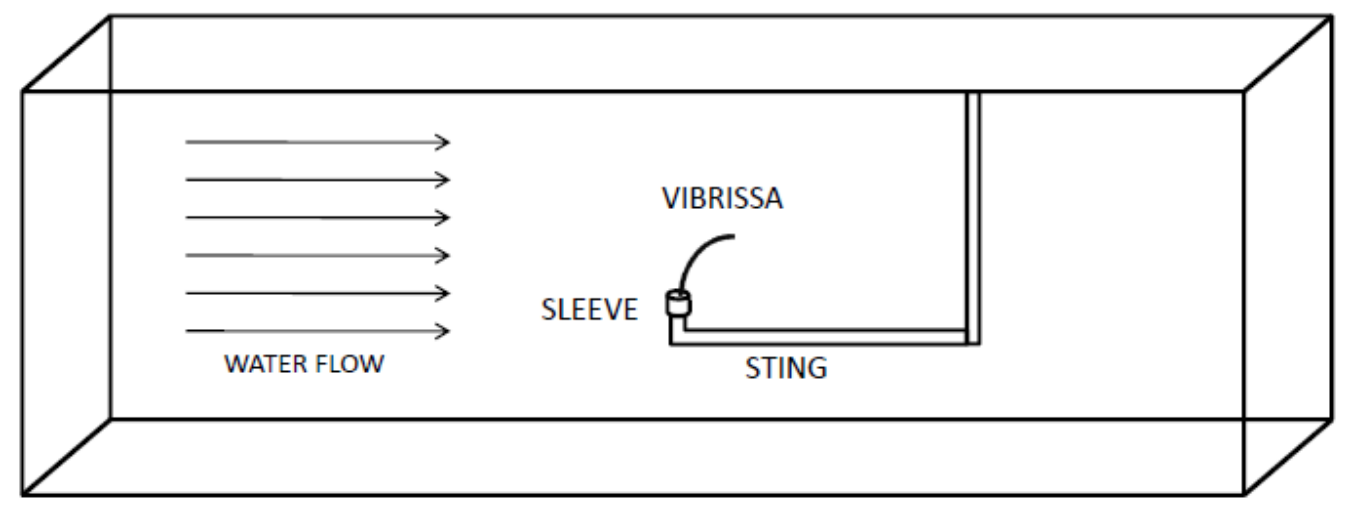

Figure 4.6: Design of tests in water flume [6].

parallel to the base of the flume. Each sample was tested individually at orientations 0, 45, and 90 degrees to the flow. Previous vibrissae vibration studies dictated the orientation by the curvature of the whisker [68]. Here the orientation was based on the natural orientation of the vibrissae of each species. This results in the whisker bending toward the downstream direction for the elephant and harbor seal specimens. The California sea lion bent in the cross stream direction. In all cases the 0 degree orientation coincided with the thin side facing the flow. As the vibrissae were rotated into the 45 and 90 degree positions, the broad edge turned to face the fluid flow.

The response of smooth and undulatory surfaced vibrissae response to obstructed flows was investigated in a manner similar to the free stream tests, however all specimens were limited to the 0 degree orientation. Here the California sea lion and harbor seal whiskers where placed in the flume for flows speeds of $2.54,5.08$, and $12.7 \mathrm{~cm} / \mathrm{s}$. The flow obstruction was a vertical cylinder in the water column $12.3 \mathrm{~cm}$ directly in front of the whisker. Cylinder diameters of $0.5,1$, and $2 \mathrm{~cm}$ generated a wake to strike the mounted vibrissae. The laser vibrometer again measured the whiskers response at the midpoint in the cross stream direction. 


\subsection{Theoretical Shedding Frequencies}

As reviewed in Chapter 2 the dynamics of a cylinder wake change with the Reynolds number. The periodic vortex shedding known as the von Karmen vortex sheet is present in flows with Reynolds number up to 270,000 [40]. Roshko [39] [38] presented a relationship between the Reynolds number and shedding frequency for cylinders given by:

$$
f=\frac{U_{H_{2} O}}{d} 0.198\left(1-\frac{19.7}{R e}\right)
$$

where Re is the Reynolds number, $U_{\mathrm{H}_{2} \mathrm{O}}$ is the fluid velocity, $\mathrm{d}$ is the cylinder diameter, $\nu$ is the fluid kinematic viscosity, and $\mathrm{f}$ is the frequency.

As previously discussed, the pinniped vibrissae are not cylindrical in shape, but elliptical in cross sectional area and taper in width and thickness from the base to the tip. The cylinder shedding frequency provided by Roshko is used as an estimation of the von Karman vortex shedding frequency of the whiskers. For the 0 degree orientation, the hair thickness is taken as the diameter. Likewise the width is used as the diameter for the 90 degree position. Assuming the width and thickness taper linearly from base to tip, the centroid of a vibrissae is located $1 / 3$ the total length from the base [91]. The values for the width and thickness used in the shedding estimation are measured at the centroid. In the case of the elephant and harbor seals which exhibit undulations along the the length, measurements were made at a crest and trough closest to the centroid with the average used to calculate Reynolds number and shedding frequency. The full range and average results of estimated shedding frequency for all three species at both orientations are presented in Table 4.2 .

Reynolds numbers ranged from 200-600 resulting in frequencies of 90-270 Hz. All three species predict a much higher shedding frequency in the 0 degree orientation. This is a direct result of the diameter being inversely proportional to frequency. The harbor seal samples predict the largest shedding frequency shift based on orientation 


\begin{tabular}{|c|c|c|c|c|c|}
\cline { 3 - 6 } \multicolumn{1}{c|}{} & \multicolumn{2}{c|}{ Frequency $(\mathrm{Hz})$} & \multicolumn{2}{c|}{ Diameter $(\mathrm{mm})$} \\
\cline { 2 - 6 } \multicolumn{1}{c|}{ Crientation } & 0 & 90 & 0 & 90 \\
\hline \multirow{3}{*}{ California Sea Lion } & Maximum & 233 & 137 & 0.38 & 0.68 \\
\cline { 2 - 6 } & Minimum & 159 & 99 & 0.58 & 0.96 \\
\cline { 2 - 6 } & Mean & 201.1 & 121.7 & 0.46 & 0.78 \\
\hline \multirow{3}{*}{ Harbor Seal } & Maximum & 201 & 111 & 0.45 & 0.86 \\
\cline { 2 - 6 } & Minimum & 154 & 94 & 0.60 & 1.02 \\
\cline { 2 - 6 } & Mean & 176.3 & 102.6 & 0.53 & 0.93 \\
\hline & Maximum & 272 & 113 & 0.32 & 0.85 \\
\cline { 2 - 6 } & Minimum & 196 & 90 & 0.47 & 1.07 \\
\cline { 2 - 6 } & Mean & 241.8 & 100.3 & 0.37 & 0.96 \\
\hline
\end{tabular}

Table 4.2: Predicted shedding frequencies of vibrissae at 0 and 90 degree orientations.

indicating the highest flattening of the vibrissae or largest width-to-thickness ratio. The ranges for each individual specie are large due to the variations in vibrissae dimensions. The values are an approximation of the vibration frequencies present in the vibrissae for a $0.5 \mathrm{~m} / \mathrm{s}$ flow caused by their own wake.

\subsection{Vibrissae Properties}

Force measurements on the samples were conducted $2 \mathrm{~cm}$ above the vibrissae base as described previously. Applying the the displacements at rates varying from 0.5 $\mathrm{mm} / \mathrm{s}$ to $2 \mathrm{~mm} / \mathrm{s}$ showed no variability in the force results. For all tests presented here the linear actuator traveled at $1 \mathrm{~mm} / \mathrm{s}$. The average force displacement results are plotted in Figure 4.7. Due to the high variation in the whisker thicknesses the resulting individual whisker results covered a large range (resulting in standard deviations up to $50 \%$ of the average applied force for a given displacement) and thus error bars are ommited from the plot.

Of importance is the nearly linear response exhibited by all three species. The elephant seal and California sea lion exhibit strikingly similar results while the harbor seal demonstrates a significantly lower stiffness. The spring constant is a measure of the force required to generate a given deflection. Here the spring constant, $S_{v i b}$. on the thin whisker edge for each specie is modeled as the ratio of the deflection, $y$, to 


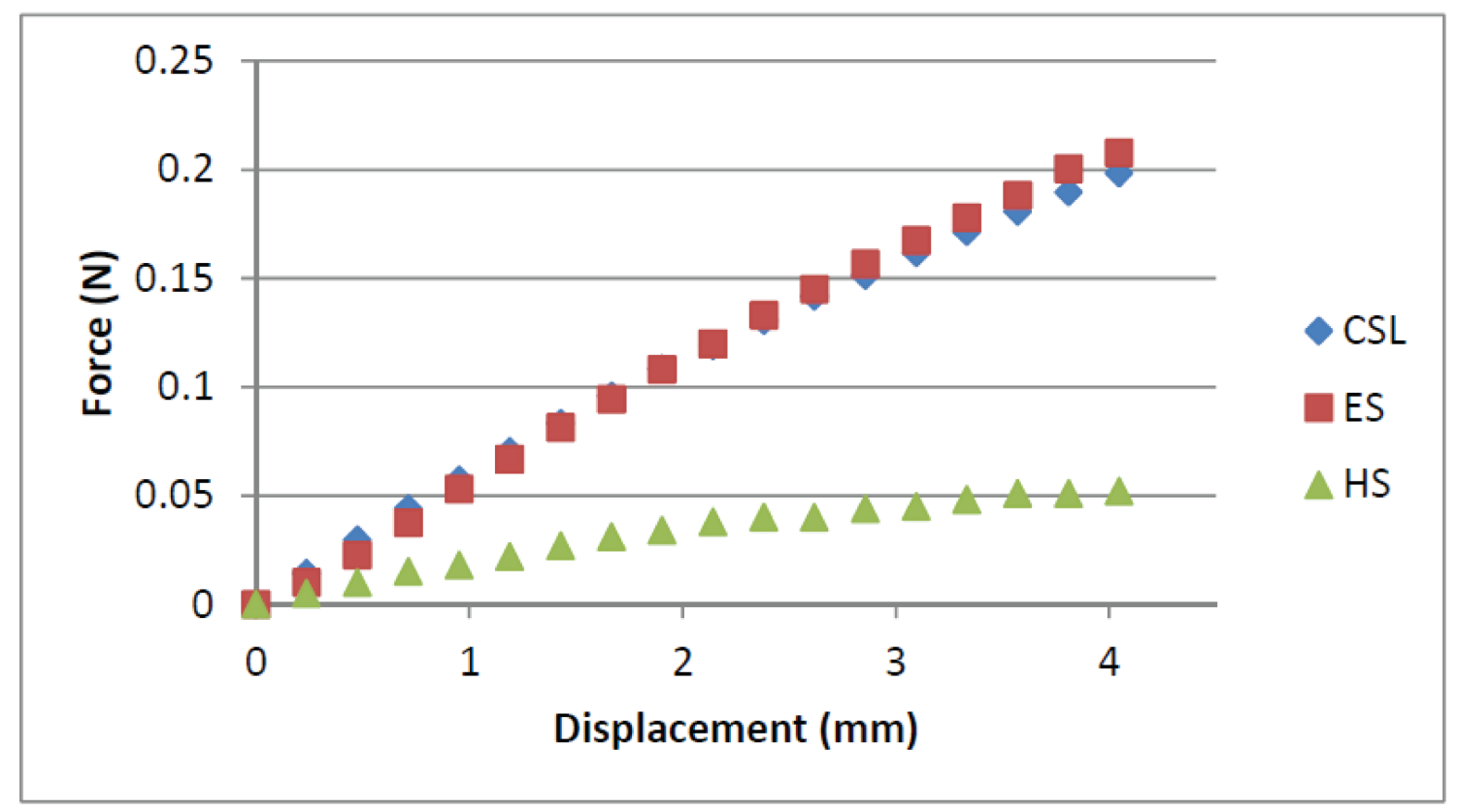

Figure 4.7: Force displacement plot for pinniped vibrissae. Forces applied $2 \mathrm{~cm}$ above the whisker base with displacements up to $4 \mathrm{~mm}$.

the point force applied, F:

$$
S_{v i b}=\frac{F}{y}
$$

This equates to the slope of a linear trend line applied to the results in Figure 4.7 . The resulting spring stiffnesses of the California sea lion and elephant seal are 52.2 $\mathrm{N} / \mathrm{m}$ and $53.7 \mathrm{~N} / \mathrm{m}$ respectively. The harbor seal vibrissae have a spring stiffness of $14.9 \mathrm{~N} / \mathrm{m}$ under a third that of the other pinniped species. In actuality the whiskers taper from the base to the tip as mentioned as evident in many mammalian species [92]. This implies the spring constants actually decrease from the base to the tip. This phenomena is demonstrated in Figure 4.8 (the force tapers off of the $1 \mathrm{~cm}$ data due to loss of contact at the large base deflection). The harbor seal vibrissae is significantly stiffer $1 \mathrm{~cm}$ from the base with a spring constant of $75.6 \mathrm{~N} / \mathrm{m}$ compared to a spring constant of $0.7 \mathrm{~N} / \mathrm{m} 5.6 \mathrm{~cm}$ from the base. The properties here are for location $2 \mathrm{~cm}$ above the vibrissae base averaged over the samples and provide a general comparison between the species. 


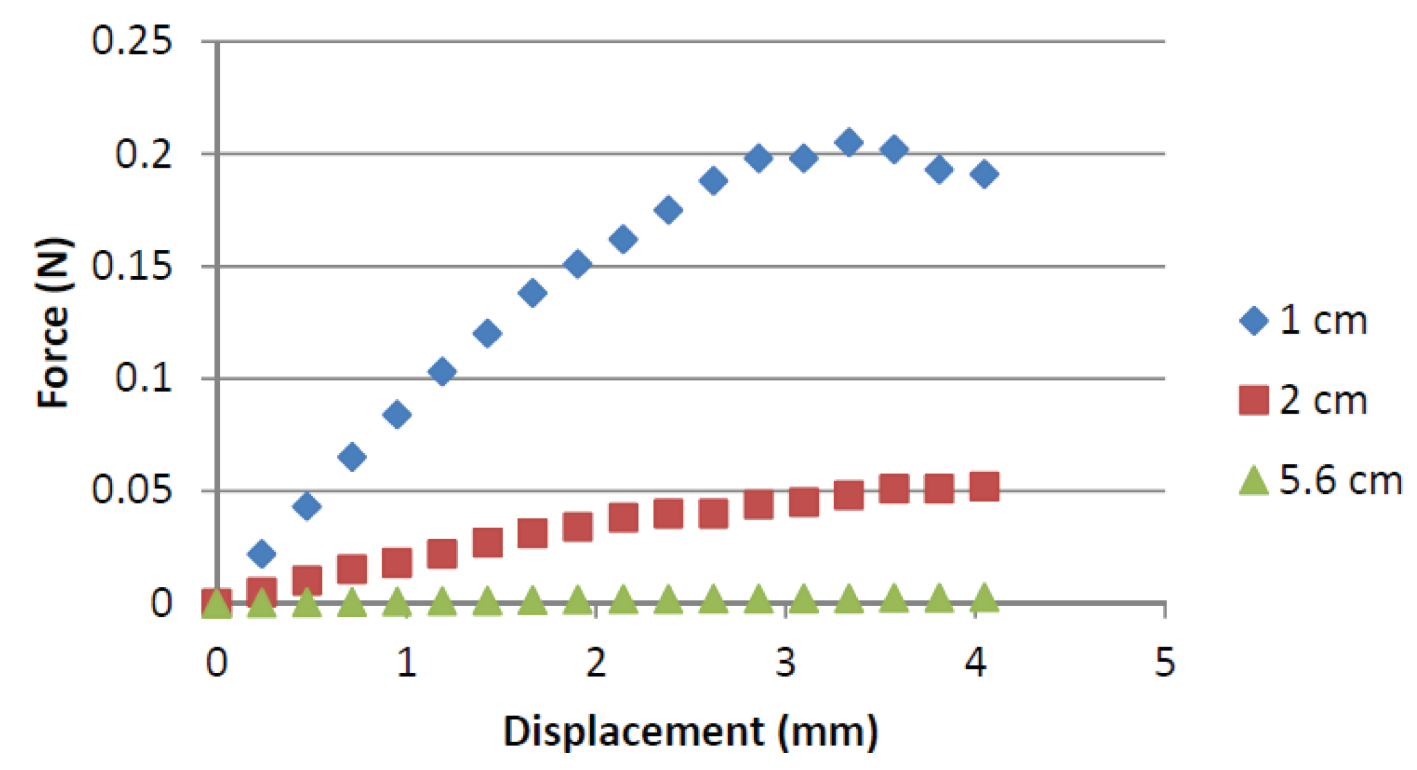

Figure 4.8: Force displacement plot along a single harbor seal vibrissae. Forces are applied 1, 2, and $5.6 \mathrm{~cm}$ from the base.

While the spring constant varies along the vibrissae length, the modulus of elasticity, E, is a description of the stiffness over the entire length assuming it is isotropic along the entire whisker. For a cantilever beam with one end fixed (epoxied to the mount) and one end free, the deformation of the beam, from the base to the load location, to a point load applied is given in Equation 4.3 ;

$$
y(x)=\frac{P x^{2}}{6 E I}(3 a-x)
$$

where $\mathrm{y}(\mathrm{x})$ is the deformation at location $\mathrm{x}$ along the beam, $\mathrm{P}$ the applied point force, E the modulus of elasticity, I the moment of inertia, and a the location of the point load. Due to the flattening of the vibrissae the cantilever is approximated as an ellipse in cross section with a moment of inertia given by:

$$
I=\frac{\pi 0.5 w \cdot(0.5 h)^{3}}{4}
$$

where $\mathrm{h}$ is the vibrissae thickness and $\mathrm{w}$ the width. The values are taken as those 
from the centroid provided in Table 4.2. The width is the diameter at 90 degrees and thickness the 0 degree diameter. From these equations the approximate Young's modulus is calculated. The modulus of elasticities are $39.8 \pm 3.0 \mathrm{MPa}, 21.0 \pm 1.5$ $\mathrm{MPa}, 19.1 \pm 3.0 \mathrm{MPa}$ for the California sea lion, elephant seal, and harbor seal respectively. The California sea lion vibrissae are the most rigid while the harbor seal vibrissae are the most prone to deformation. The modulus of elasticity is a helpful characterization of the material and allows for easy comparison to other vibrissae. Tactile whiskers such as those in rats have a modulus of elasticity ranging from 3-4 GPa [93] [94].

Natural frequencies and damping constants of the samples were made by deflecting the the whisker and allowing it to vibrate freely to rest. The whisker motion was measured with the laser vibrometer at $50 \%$ the whisker length. The forced displacement to free vibration generates the first mode resonant frequency. A typical measurement is shown in Figure 4.9. The velocity oscillates from a starting speed of around $40 \mathrm{~mm} / \mathrm{s}$ at a constant frequency decreasing over time until the whisker comes to a rest. The amplitude decreases over time in a logarithmic fashion. All vibrissae demonstrated this type of magnitude reduction for each successive oscillation. This indicates the vibrissae from all three species are underdamped and experience a viscous damping of the free vibration [95].

Taking the fast fourier transform (FFT) of the vibration motion generates the frequency response. The FFT of C65 is provided in Figure 4.10. A distinct peak is visible at $75.9 \mathrm{~Hz}$. All samples also demonstrated a frequency response with a single peak frequency of vibration. Typical resonant frequencies decrease inversely with length (provided all other dimensions are constant). No trends with whisker length were apparent in the free vibration resonant frequencies. This is attributed to variations in width, thickness, and undulatory pattern of the vibrissae. The resonant frequency measured is actually the damped natural frequency and is related to the natural frequency by: 


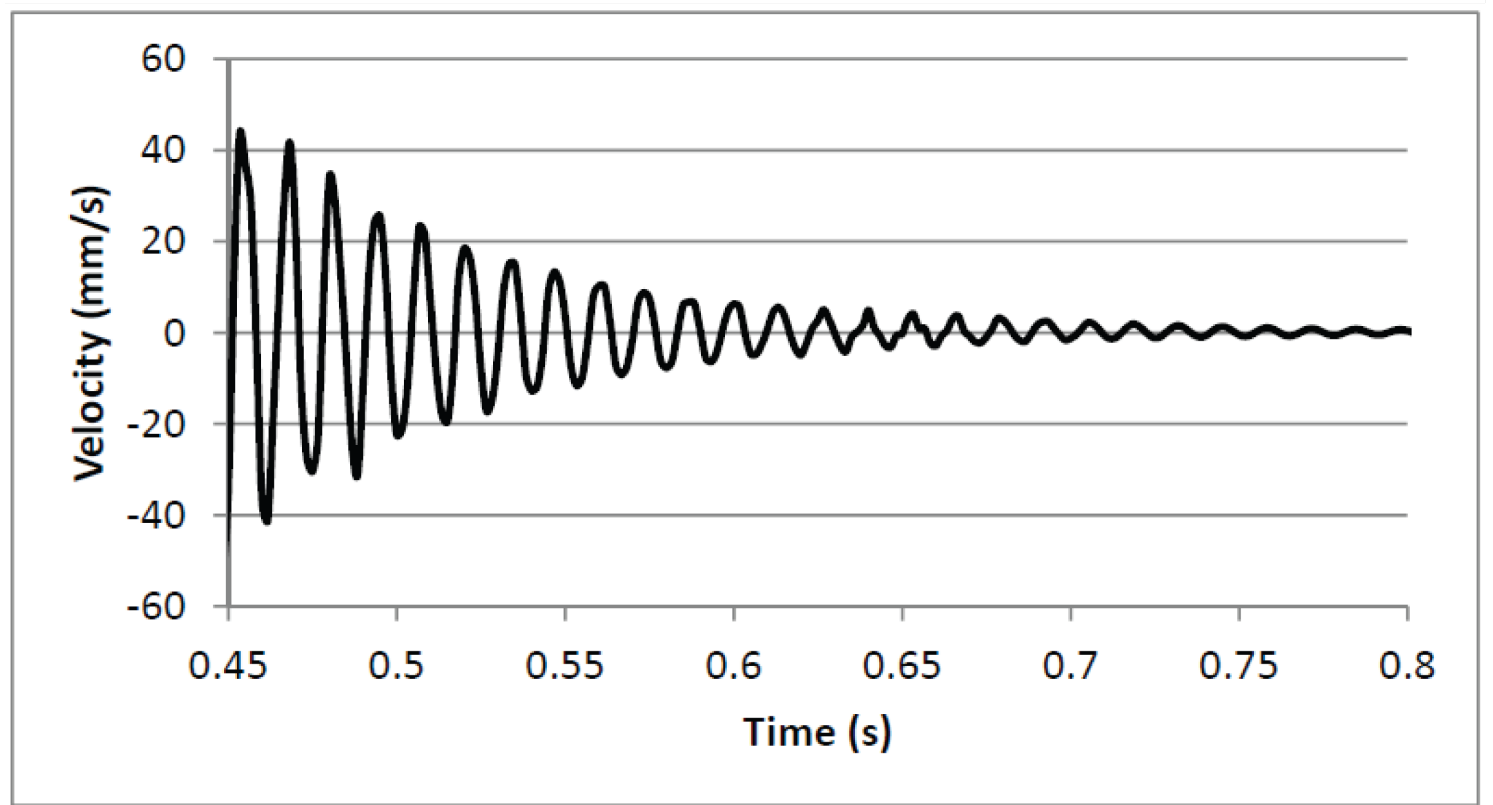

Figure 4.9: Free vibration of California sea lion vibrissae specimen C65.

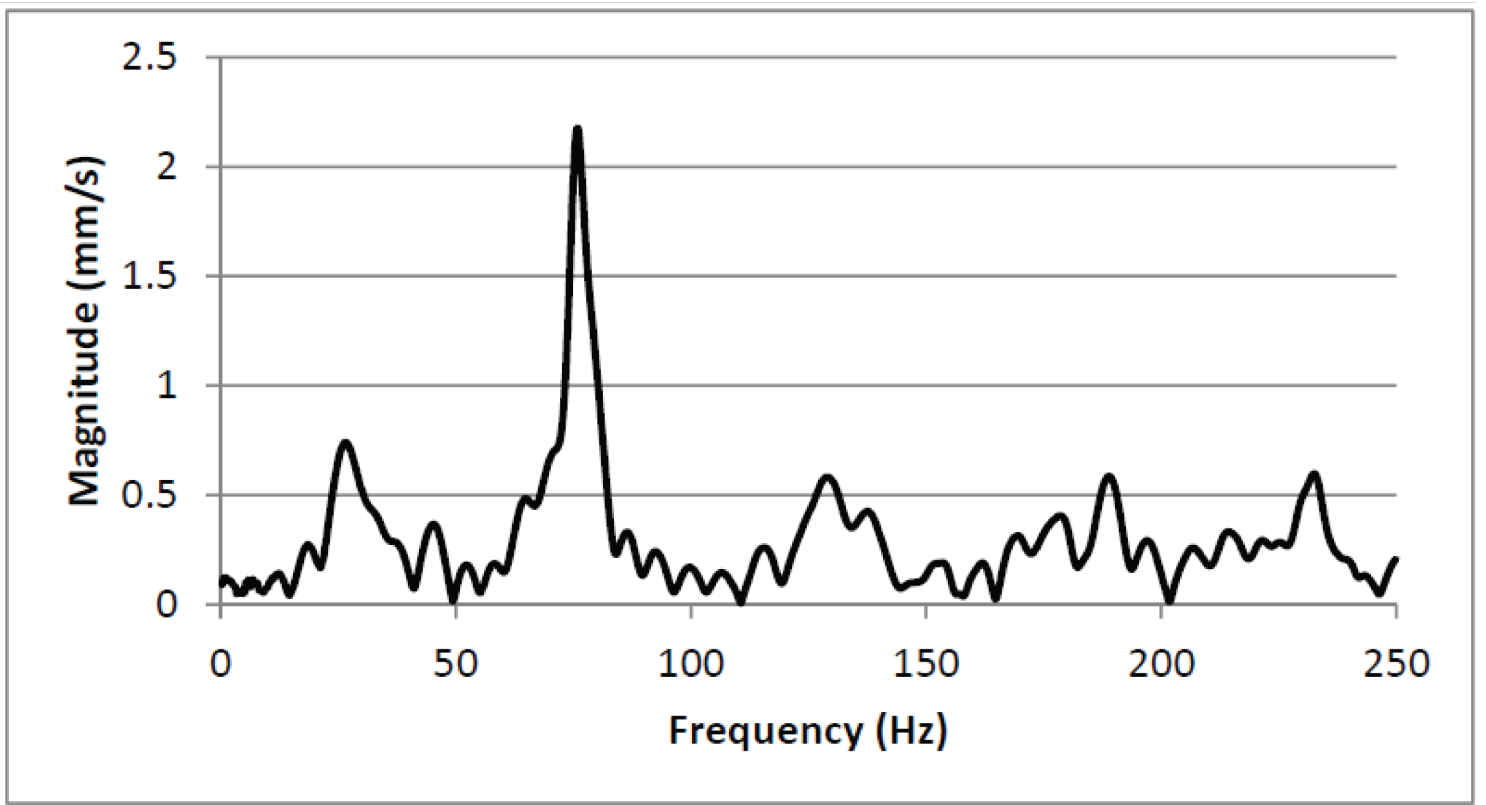

Figure 4.10: Frequency response of free vibration of California sea lion vibrissae specimen C65. 


\begin{tabular}{|c|c|c|c|}
\hline Animal & $\omega_{d}(\mathrm{~Hz})$ & $\omega_{n}(\mathrm{~Hz})$ & $\zeta$ \\
\hline California sea lion & $63.5 \pm 12.7$ & $63.5 \pm 12.7$ & $0.029 \pm 0.0055$ \\
\hline Elephant seal & $81.7 \pm 12.4$ & $81.8 \pm 12.4$ & $0.028 \pm 0.0039$ \\
\hline Harbor seal & $45.1 \pm 9.3$ & $45.2 \pm 9.3$ & $0.046 \pm 0.0080$ \\
\hline
\end{tabular}

Table 4.3: Characteristic vibration properties of vibrissae

$$
\omega_{d}=\sqrt{1-\zeta^{2}} \omega_{n}
$$

where $\omega_{d}$ is the damped natural frequency, $\omega_{n}$ the natural frequency, and $\zeta$ the damping ratio. The logarithmic decrement, $\delta$, of the oscillation is is change in amplitude between peaks and measured by:

$$
\delta=\ln \left[\frac{X_{1}}{X_{2}}\right]
$$

where $X_{1}$ and $X_{2}$ are consecutive peak amplitudes. For damping ratios under 0.3 the damping ratio is approximately $\frac{\delta}{2 \pi}$ [95]. Full average results for the damped natural frequency, natural frequency, and the damping ratio are provided in Table 4.3 .

All the pinniped species' vibrissae are very underdamped with damping ratios under 0.05. The frequency of damped harmonic motion is always less than that of the undamped natural frequency. This holds true, however due to the extremely small damping ratios, the undamped and damped natural frequencies are nearly identical. For comparison, tactile vibrissae of rats exhibit a damping ratio between 0.11 and 0.17 [93] while the damping ratio of a musical instrument is on the order $2-5 \times 10^{-5}$.

\subsection{Free Flow Results}

All samples vibrated with a distinct frequency at each orientation. The peak frequency decreased as the orientation moved from 0 to 90 degrees. However, the magnitude of the peak frequency increased significantly. Figure 4.11 shows typical responses from a sample from each specie. The vibrissae vibration is minimal in the 

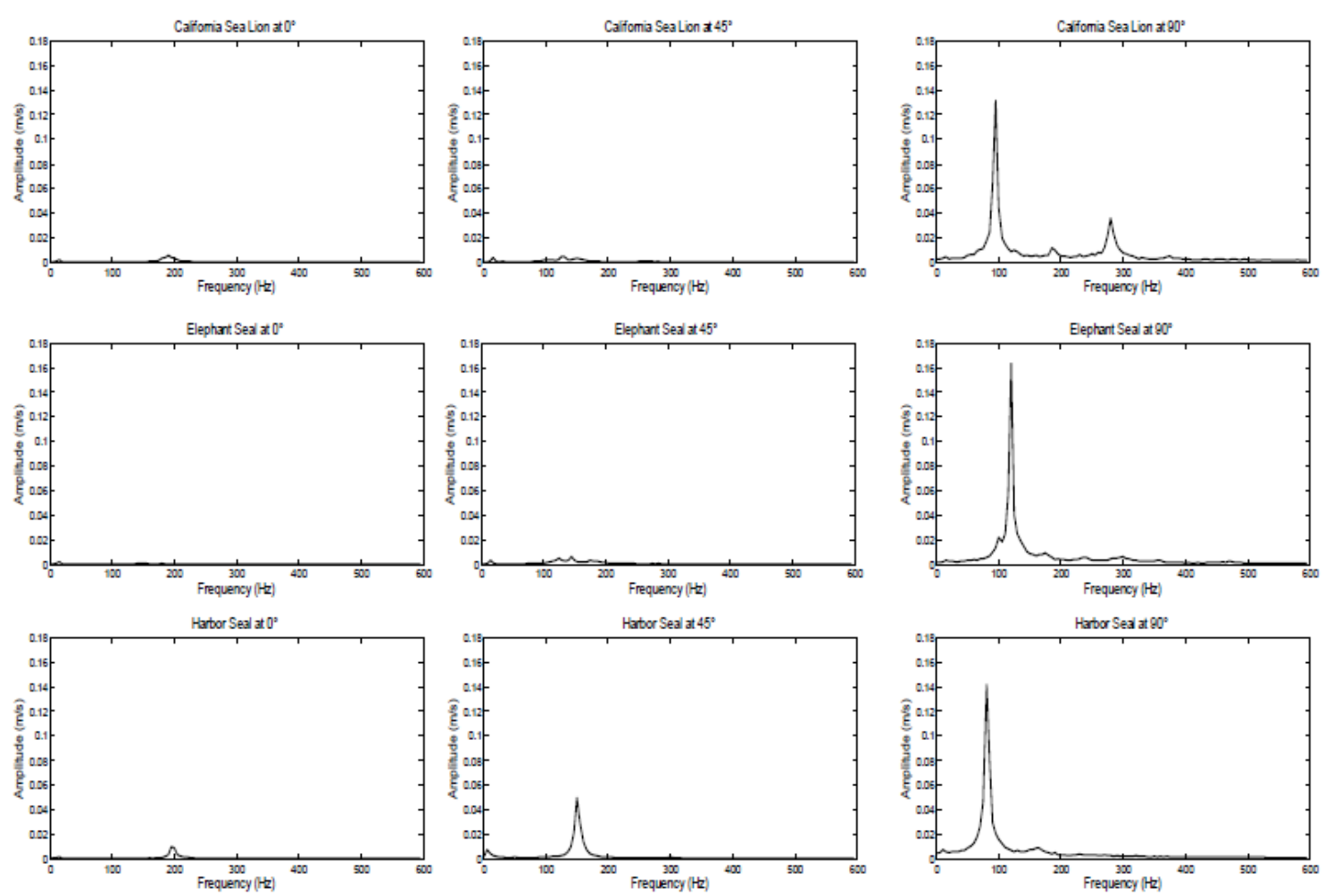

Figure 4.11: Frequency response of free vibration for California sea lion (top), elephant seal (middle) and harbor seal (bottom) for 0 degree (left), 45 degree (middle), and 90 degree (right) orientations [6].

0 degree orientation compared to the 90 which shows a significant increase in the amplitude of vibration. This suggests the whiskers are best suited to supply information to the animal in the 0 degree position coinciding with the actual orientation in the animal. Frequency responses of the entire sample set are provided in Table 4.4 .

Of particular interest are the similarities among the specie groups. Both the vibration frequencies and amplitudes provided similar values at each orientation for all whisker types. The overall peak frequencies ranged form 70-270 Hz. This range is similar to the predicted values of frequency shedding for the whiskers as previously discussed. Figure 4.12 plots the resulting peak frequency from the flume study with an uncertainty of one standard deviation. The harbor seal whiskers exhibited the largest frequency change due to orientation, however the results were similar to the California sea lion. 


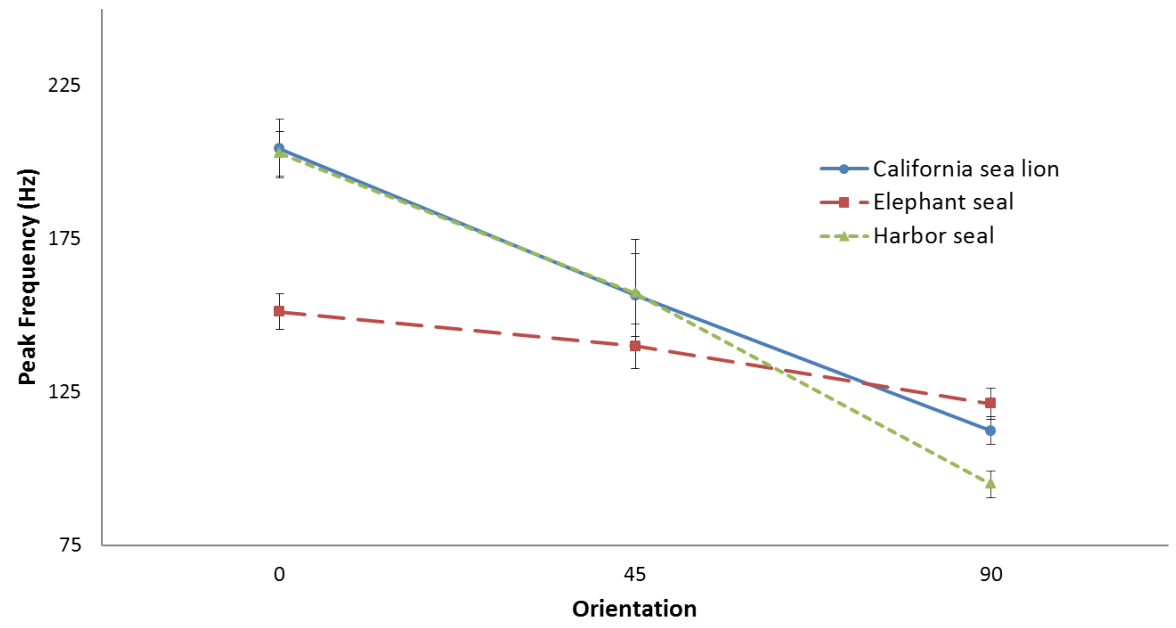

Figure 4.12: Average peak frequency for each orientation

\begin{tabular}{|c|c|c|c|c|}
\hline & & \multicolumn{3}{|c|}{ Frequency $(\mathrm{Hz})$} \\
\hline & Orientation & 0 & 45 & 90 \\
\hline \multirow{3}{*}{ California Sea Lion } & Range & $175-270$ & $115-230$ & $95-130$ \\
\hline & Mean & 204.4 & 156.7 & 112.5 \\
\hline & Error & 9.4 & 13.4 & 4.6 \\
\hline \multirow{3}{*}{ Elephant Seal } & Range & $130-185$ & $115-170$ & $105-140$ \\
\hline & Mean & 151.3 & 140 & 121.3 \\
\hline & Error & 5.9 & 7.3 & 5.2 \\
\hline \multirow{3}{*}{ Harbor Seal } & Range & $175-240$ & $70-245$ & $80-110$ \\
\hline & Mean & 202.8 & 157.2 & 95 \\
\hline & Error & 7.3 & 17.6 & 4.3 \\
\hline
\end{tabular}

Table 4.4: Vibrissae motion in $0.5 \mathrm{~m} / \mathrm{s}$ free stream flow. 


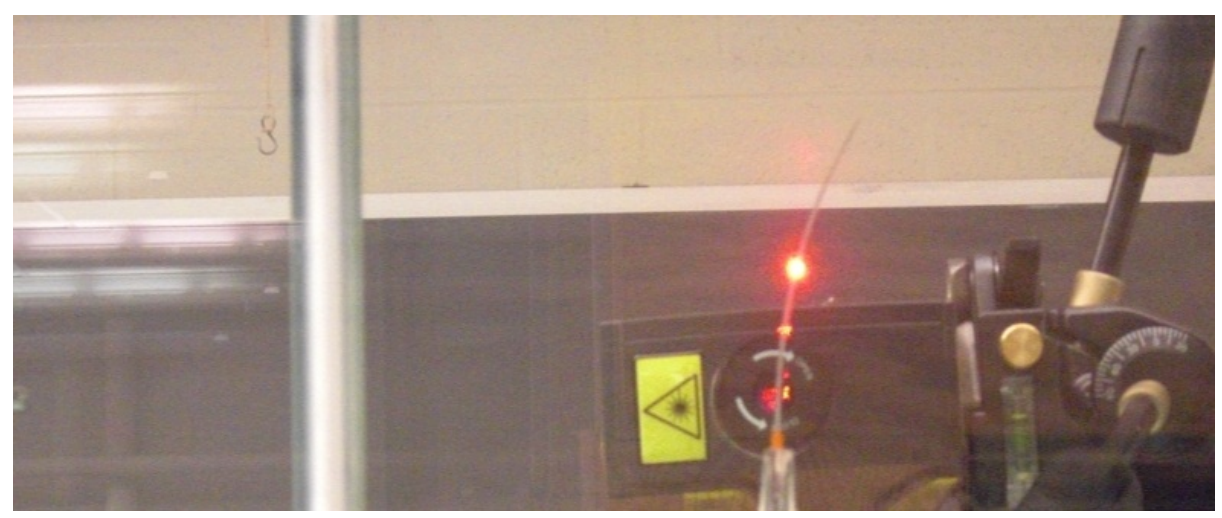

Figure 4.13: Harbor seal whisker behind $2 \mathrm{~cm}$ cylinder with laser vibrometer measuring at midpoint.

\subsection{Obstructed Flow Results}

The obstructed flow tests were designed to compare the response of smooth (California sea lion) and undulated (harbor seal) vibrissae to a wake with a known characteristic frequency. Cylinders with diameters of $0.5,1$, and $2 \mathrm{~cm}$ provided the obstruction and as previously discussed, the cylinder wake has a distinct frequency characteristic. The cylinder spanned the entire water column and was placed $12.3 \mathrm{~cm}$ directly upstream of the vibrissae as shown in Figure 4.13.

Following the same methodology as described in the Theoretical Shedding Frequencies section, it is possible to predict the von Karman vortex sheet shedding frequency for the test cases. Flow speeds for each cylinder were 2.54, 50.8, and 12.7 $\mathrm{cm} / \mathrm{s}$. This results in cylinder Reynolds numbers ranging from 127 to 2500. The resulting frequencies of the cylinder wakes range from 0.27 to $5.3 \mathrm{~Hz}$. Table 4.5 shows the frequency of the cylinder wakes for all nine test cases. It is noted that these frequencies are much lower than those of the vibrissae in the free stream flow at 50 $\mathrm{cm} / \mathrm{s}$.

For each test, a baseline measurement of the vibrissae in the flow without the the cylinder was made to compare the frequency response of the whisker's inherent vibration to that when the cylinder wake is present. An example of this result is shown in Figure 4.14 for the case of the $1 \mathrm{~cm}$ cylinder in a $5.08 \mathrm{~cm} / \mathrm{s}$ flow. As expected from 


\begin{tabular}{|c|c|c|c|}
\hline & $2.54 \mathrm{~cm} / \mathrm{s}$ & $5.08 \mathrm{~cm} / \mathrm{s}$ & $12.7 \mathrm{~cm} / \mathrm{s}$ \\
\hline $0.5 \mathrm{~cm}$ cylinder & 1.07 & 2.13 & 5.3 \\
\hline $1 \mathrm{~cm}$ cylinder & 0.53 & 1.07 & 2.7 \\
\hline $2 \mathrm{~cm}$ cylinder & 0.27 & 0.53 & 1.3 \\
\hline
\end{tabular}

Table 4.5: Expected cylinder wake frequencies (Hz).
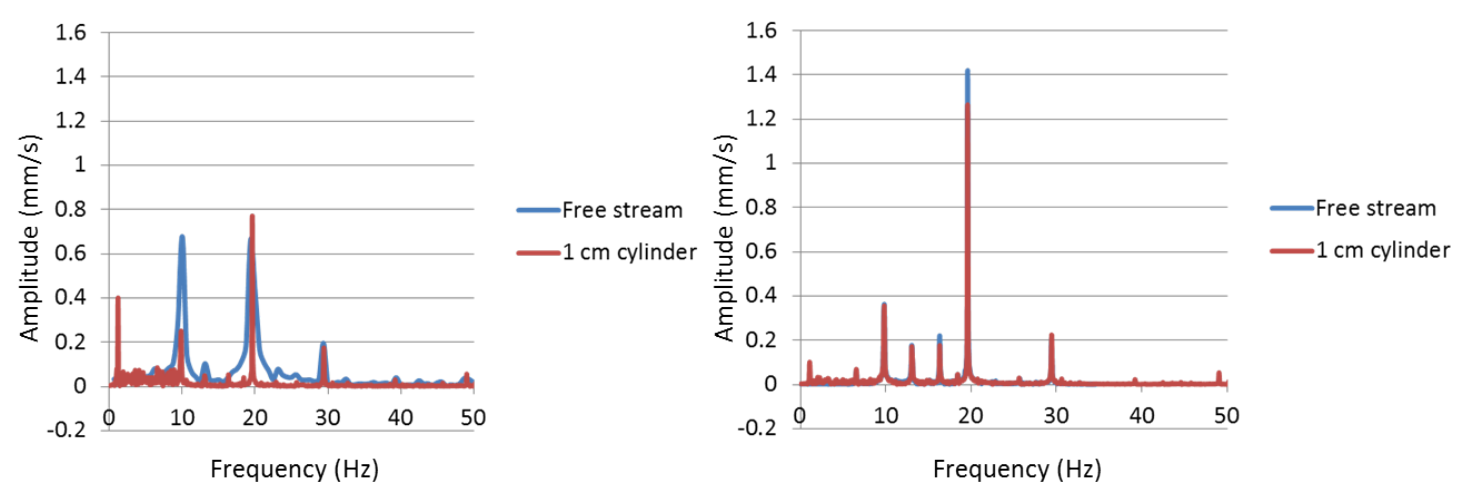

Figure 4.14: Harbor seal (left) and California sea lion (right) in free flow (blue) and in presence of $1 \mathrm{~cm}$ cylinder (red) in a $5.08 \mathrm{~cm} / \mathrm{s}$ flow. Note the emergence of a $1 \mathrm{~Hz}$ peak in the cylinder wake case (red).

the free flow tests, each vibrissae moves at a distinct frequency in a clean flow. Adding the $1 \mathrm{~cm}$ cylinder upstream, the frequency peaks remain but a new frequency appears at $1 \mathrm{~Hz}$. This corresponds to the shedding frequency of the cylinder wake. Frequency information in the obstructed flow can be found by subtracting the FFT cylinder response from the FFT of the free stream vibration.

As shown in Figure 4.14 both the harbor seal and California sea lion vibrissae detect a $1 \mathrm{~Hz}$ signal. However the strength of this detection is different for the two whisker types. The $1 \mathrm{~Hz}$ peak has a much larger amplitude with the harbor seal than the California sea lion. This was evident in all test cases. For the lowest flow speed of $2.54 \mathrm{~cm} / \mathrm{s}$ no cylinder frequencies were detected by the California sea lion vibrissae for all three cylinders. The harbor seal was still able to detect a new frequency in these test cases. The full results are plotted in Figure 4.15. The variation in detected frequencies was under $2 \%$ for all vibrissae. Both the smooth and undulated vibrissae detected matching frequencies with the exception of the California sea lion at the 


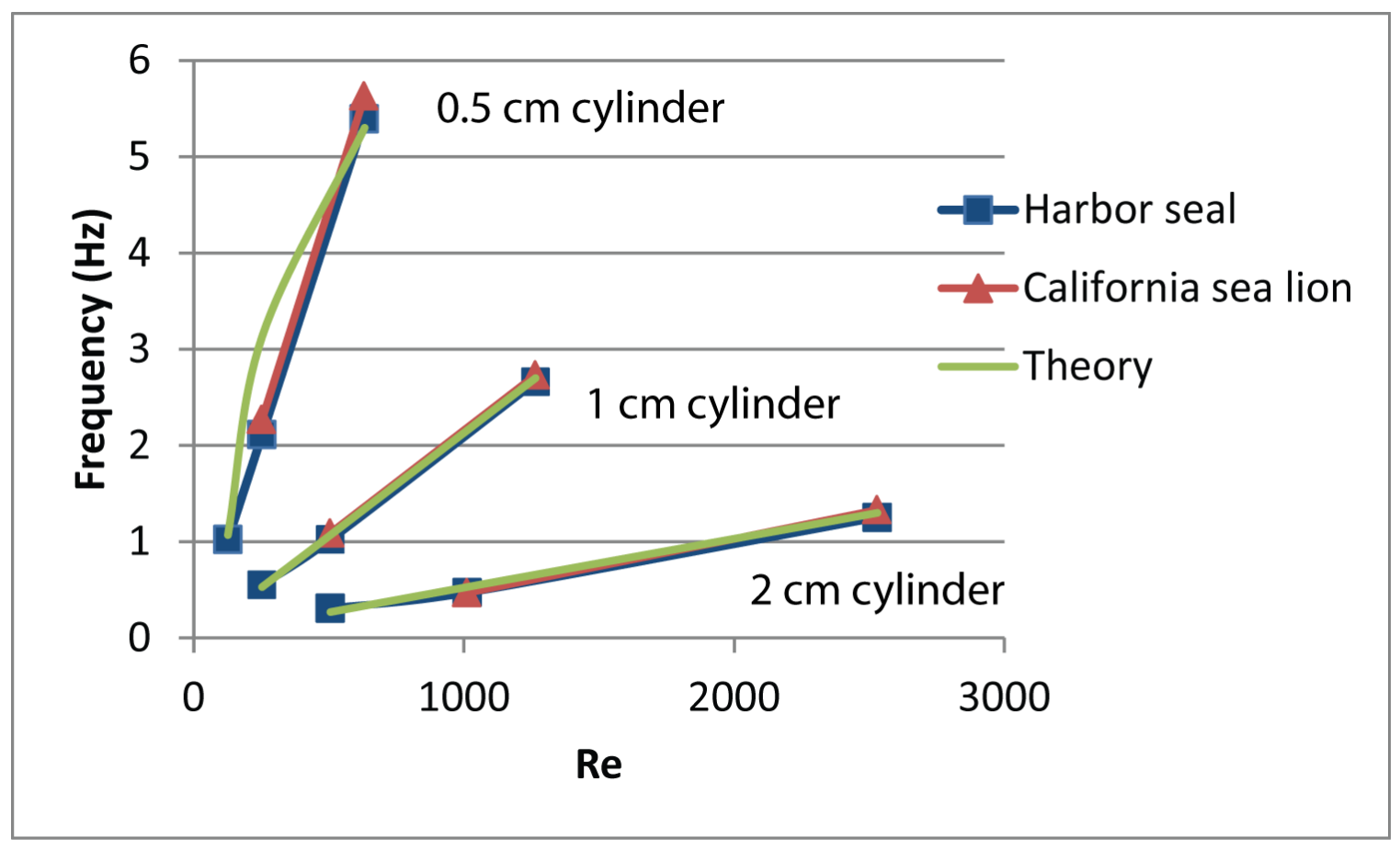

Figure 4.15: Peak frequencies detected and theoretical shedding frequencies of a cylinder wake for harbor seal and California sea lion vibrissae.

lowest flow speed where no peak frequencies were evident in the data. The vibration frequencies of the vibrissae match those of the predicted vortex shedding frequecnies of the cylinder wake. It is apparent that the vibrissae are capable of correctly capturing the wake hydrodynamics of the cylinder.

The Strouhal number is a dimensionless number describing oscillating flow mechanisms. For the cylinder case the Strouhal number is:

$$
S t=\frac{f d}{U}
$$

where St is the Strouhal number, $\mathrm{U}$ the fluid velocity, and $\mathrm{d}$ the cylinder diameter. For the cylinder flows presented here the accepted Strouhal number is 0.21. Plotting the Strouhal number of the frequencies detected by the vibrissae against Reynolds number is shown in Figure 4.16. The results show good agreement with the Strouhal number of 0.21 for all test cases.

Overall the vibrissae captured the shedding frequency of the cylinder wake. The 


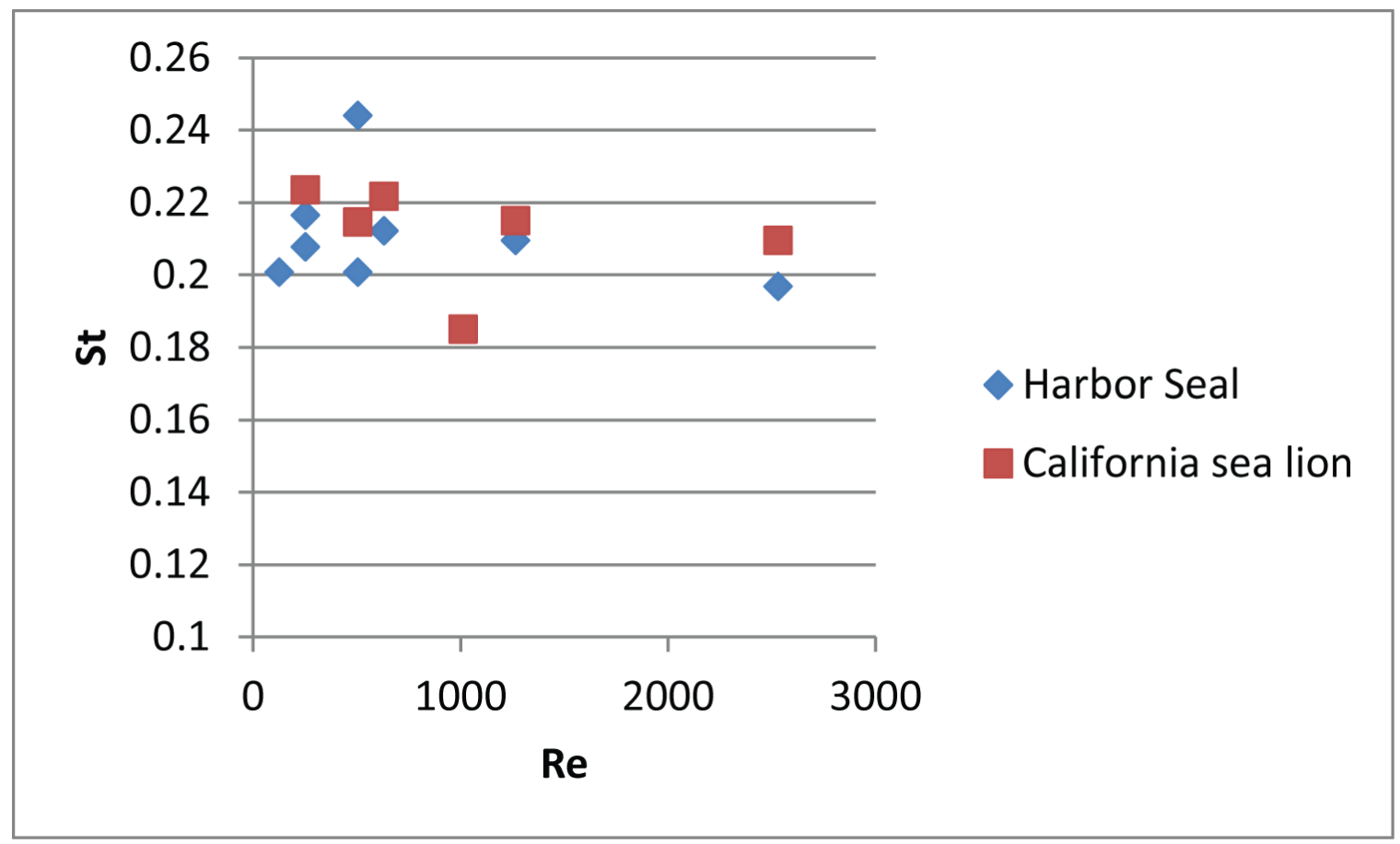

Figure 4.16: Nondimensional results of vibrissae detection of cylinder wakes.

harbor seal vibrissae demonstrated a stronger response than that of the California sea lion. The smooth vibrissae were also unable to detect the wake at the lowest flow speeds.

\subsection{Discussion}

To the author's knowledge the results presented here for the material properties of pinniped vibrissae have never been examined. Large variations were present in the data caused by the differences in dimensions within each specie sample set. The samples were chosen solely by length. Despite similar lengths (although the range covered over $2 \mathrm{~cm}$ difference between the shortest and longest) the vibrissae possessed a variety of widths and thicknesses causing the large standard deviations in the force-displacement measurements. Vibrissae stiffnesses at a location $2 \mathrm{~cm}$ above the whisker base demonstrated similar values between the California sea lion and elephant seal, however the harbor seal revealed a stiffness value about $70 \%$ less. The 
Young's modulus showed greater variability between the species. The Elephant seal had modulus of elasticity about half that of the California sea lion while the harbor seal again was much lower with a modulus only about $48 \%$ that of the California sea lion. Modeling the vibrissae as an isotropic linear elastic beams introduces some error in this property as most biological materials are likely to be anisotropic and thus these values are approximations. Uncertainty was also introduced in the calculation of the moment of inertia. The whiksers taper and the use of the centroid dimensions are again an approximation of the full characteristic dimensions. However these values imply the harbor seal is more sensitive to forces and therefore hydrodynmic stimulus than the the other animals.

In free vibration the vibrissae each moved at a single frequency as expected from a pinged cantilever. Here the elephant seal had the highest natural frequency at 81.7 $\mathrm{Hz}$ followed by the California sea lion at $63.5 \mathrm{~Hz}$ and then the harbor seal at 45.1 Hz. All three species were underdamped allowing the whisker to vibrate back and forth before coming to rest. The low damping ratios resulting in the undamped natural frequency being nearly equivalent to the damped. This damping ratio is 2-5 times lower than that of tactile land based mammalian vibrissae. This indicates the vibrissae are more susceptible to continued vibration in the presence of an oscillating hydrodynamic stimuli and may play a factor the animal's ability to track wakes.

Both the smooth and undulated vibrissae showed similar ranges in frequency and amplitude when in an unobstructed flow. The free flow tests proved the vibrissae are most stable in their 0 degree orientation corresponding to the natural position on the animal. The frequency of vibration decreased as the whiskers moved to the 90 degree position and the wider side of the hair faced the flow. Likewise the amplitude of vibration increased as the broad side of the of whisker turned to face the flow. The predicted values of vortex shedding frequencies assuming the vibrissae were cylinders were in good agreement with the actual vibration of the vibrissae at the 0 and 90 positions. This implies the motion of the vibrissae was caused by its own wake through 
vortex induced vibration. This is in agreement with previous studies by Hanke et al [67]. However contrary to prior studies the undulatory vibrissae did not show a reduction in amplitude at the 0 degree position. This may be due to two important factors. Previous testing by Hanke et al [68] finding a reduction in vibration amplitude in a free flow between harbor seal and California sea lion vibrissae used the whisker curvature to dictate orientation. The California sea lion whiskers naturally curve downward compared to a backward curvature of the harbor seal vibrissae. Thus the 90 degree position of the California sea lion in this work corresponds to the orientation by Hanke et al. Another factor limiting the undulatory effect may be the flow speed. The tests here were all conducted at $0.5 \mathrm{~m} / \mathrm{s}$ slow compared to the swimming speed of pinnipeds (up to $4 \mathrm{~m} / \mathrm{s}$ ).

The largest difference between the undulated and smooth vibrissae was evident in the obstructed flow tests. Both types of vibrissae accurately captured the vortex sheet frequency from a cylinder wake, however the magnitude of the detection was stronger in the undulated harbor seal vibrissae. The California sea lion was unable to detect any of the cylinders' wakes at the lowest flow velocity. While the difference in the results of the two species may be partly attributed the the differences between the morphologies, it is likely due to the lower stiffness in the harbor seal whiskers allowing for more deflection to smaller fluid forces. Similar tests by Miersch et al [65] demonstrated similar capabilities between the two species however the frequency of the vibrissae differed by as much as $30 \%$ from the cylinder shedding. The California sea lion was also unable to detect a $1.6 \mathrm{~cm}$ cylinder in flows under $18 \mathrm{~cm} / \mathrm{s}$. The tests presented here show significantly better correlation between the vibrissae response and shedding frequency likely due to better mounting techniques and measurement precision through the use of the laser vibrometer. 


\subsection{Summary}

The goal of this chapter was to develop material properties and characteristics of pinniped vibrissae to free and obstructed flows. The California sea lion and elephant seals had comparable spring constants while the harbor seal's spring constant was less than a third that of the other two. The Young's modulus of elasticity was nearly $40 \mathrm{MPa}$ in the California sea lion, while it was about half that in the elephant seal and harbor seal. All three pinniped species had underdamped vibrissae with natural frequencies ranging from 45-82 Hz. The whiskers also exhibited similar trends when placed in a $0.5 \mathrm{~m} / \mathrm{s}$ clean flow. This indicates the oval cross sectional shape of the whiskers plays a larger role than the undulatory morphology in the vibrissae response under the test conditions. Likewise both smooth and undulated vibrissae were able to capture cylinder wake shedding frequency although the undulated harbor seal vibrissae showed higher amplitudes in the frequency response. 


\section{Chapter 5}

\section{Haircell Sensor Modeling}

Pulling inspiration from the hair sensors found in fish and spiders this chapter examines the capabilities of a microelectromechanical systems (MEMS) sensor designed to detect disturbances in flow fields. The trichobothria of spiders do not bend or deflect when struck with a fluid force and only rotate about the base. The hairs in fish neuromasts bend when placed under fluid forces. Combining these features a MEMS sensor devised of a cantilever beam aligned with the body surface or parallel to the fluid motion and with a nondeforming post at the end is investigated. The fluid motion contacts the post generating a moment on the cantilever which in turn bends. The magnitude of the bend is a measure of the fluid forces acting on the sensor. The goal of the research effort in this chapter is to develop the necessary equations of motion to model the haircell sensor. By combining the kinematics of the sensor with the fluid forces of a prescribed flow, a fluid-structure interaction code is developed to capture the sensor response. Through modeling the system response to real flow conditions, the parameters of the sensor can be adjusted in a more time and cost effective manner than building and testing individual sensor designs. The code also provides insight into the types of fluid motion capable of being monitored with this style sensor. The work answers the questions regarding what sensor design parameters most affect the sensitivity of the haircell and what output is to be expected 


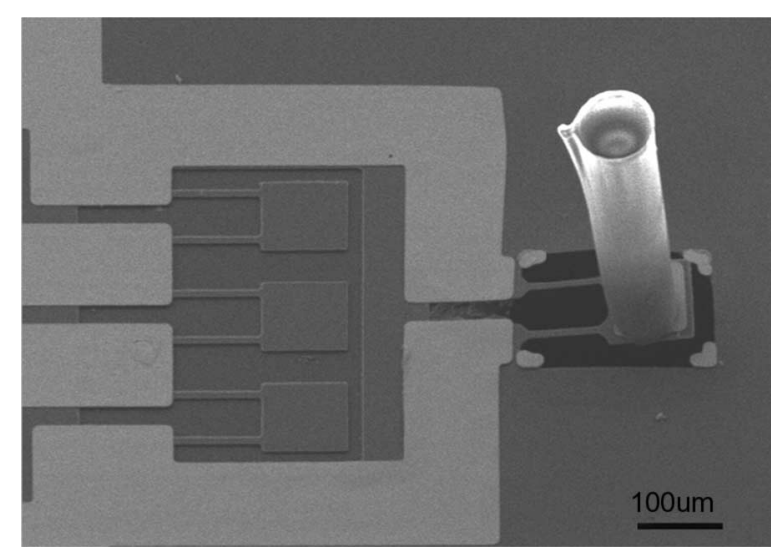

Figure 5.1: Scanning electron micrograph of artificial haircell sensor [7].

from oscillating flows. The major contribution of this chapter is the development of a fluid-structure interaction code modeling the cantilever sensor, which ties into the thesis statement by evaluating this sensor in its ability to track hydrodynamic signals. Research questions answered in this chapter include:

- How do design parameters affect sensor response?

- How does the sensor respond to prescribed flows?

- What are design limitations of this sensor?

\subsection{Cantilever Fluid Motion Sensor}

The haircell sensor consists of silicon cantilever beams with a vertical cylindrical SU-8 epoxy post fixed on the tip (Figure 5.1). The beam itself consists of two, parallel, narrow beams. The post rests on a paddle extension at the end of the cantilever beams. The sensor lies along the body of an object and is small enough to be constructed into arrays. Fluid motion passing the sensor places force on the vertical post causing the beam to bend. Measurement of this deflection is measured via piezoresistive strain gauges located at the base of the beam in a single direction. The haircell sensor captures the fluid motion in a single axial direction. The goal of the sensor is to monitor low frequency turbulence with low velocity amplitude [7]. 


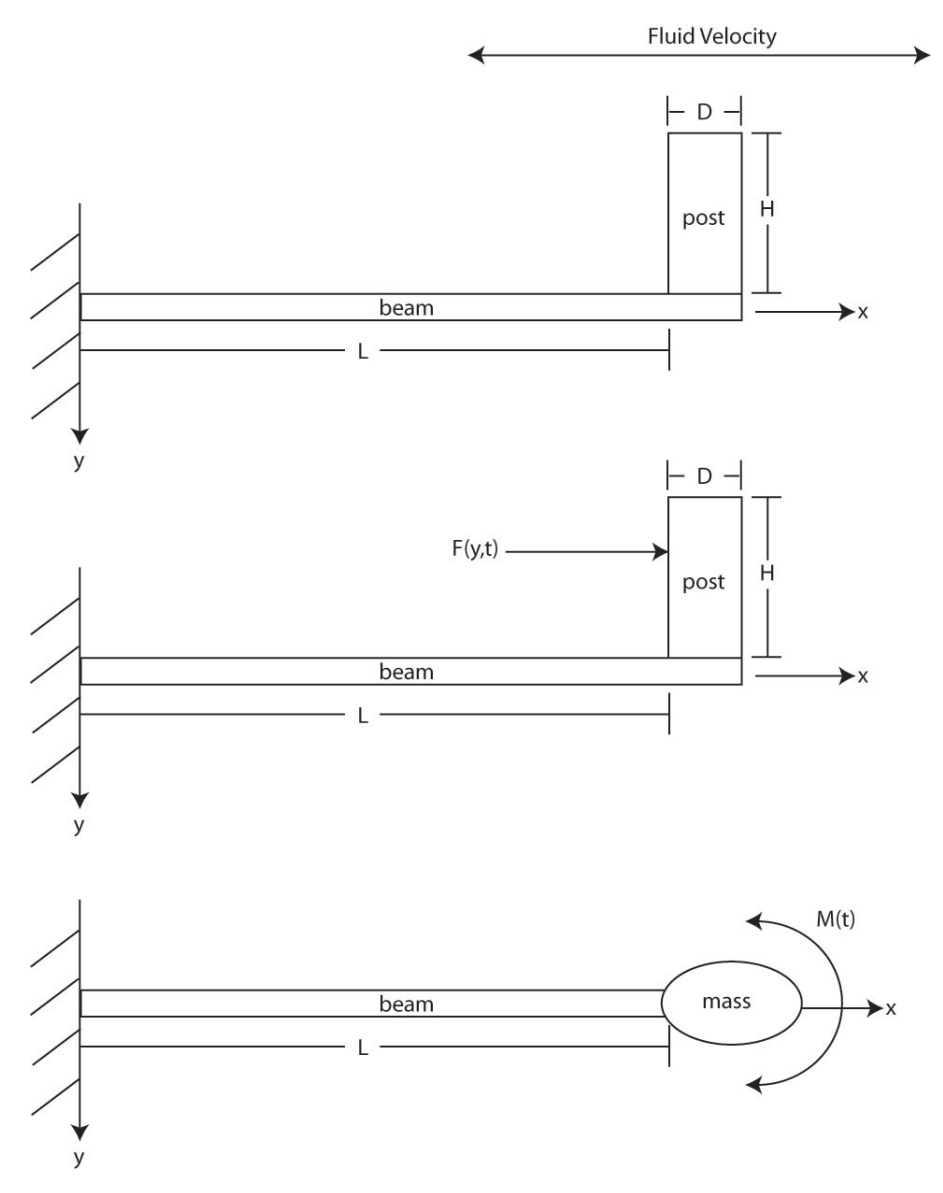

Figure 5.2: Simplification of haircell sensor to a cantilever beam fixed and one end and with a concentrated mass and moment acting on the free end.

\subsection{Modeling Sensor}

\subsubsection{Simplification}

The sensor modeling effort focuses on generating a simple fluid-structure interaction solution to predict the haircell sensor response to various simple fluid flows under changing size criteria in the sensor dimensions. The prescribed fluid flow is parallel to the cantilever beams and initially strikes the post perpendicular to the fluid velocity. The fluid is moving parallel to the beams resulting in no torsional forces on the sensor. Thus the only force causing deflections are the fluid forces applied to the post. These can be simplified to a single point force acting on the post. This point force is then resolved to a moment acting on the free end of the cantilever. The paddle 
supporting the post and the post itself are grouped together as a concentrated mass also located at the free end of the cantilever. The two parallel beams forming the cantilever are coupled into a single beam having twice the width of an individual one. The beam base is attached to a fixed rigid support. The haircell sensor reduces to a simple cantilever beam with one end fixed and the other consisting of a concentrated mass with a moment load. The simplification is illustrated in Figure 5.2 .

\subsubsection{Governing Equations}

The motion of the fluid medium about the post generates a moment at the tip of the cantilever beam that results in the deflection of the beam as measured by a strain gage. The sensor is modeled analytically using Euler-Bernoulli beam theory for a linear, elastic, isotropic, homogeneous material (Equation 5.1) subject to appropriate boundary conditions to determine deflections of the beam due to the flow field.

$$
\frac{\partial^{2}}{\partial x^{2}}\left(E I \frac{\partial^{2} y}{\partial x^{2}}\right)+\rho_{\text {beam }} A_{\text {beam }} \frac{\partial^{2} y}{\partial t^{2}}=q(x, t)
$$

In these equations $\mathrm{E}$ (Young's modulus), I (moment of inertia), $A_{\text {beam }}$ (crosssectional area), L (length) and $\rho_{\text {beam }}$ (cantilever beam density) are beam parameters. $\mathrm{x}$ is the distance along the beam and $\mathrm{y}$ is the transverse direction (direction of beam deflection). $q(x, t)$ is the forcing function or forces acting on the cantilever body. In this case $\mathrm{E}$ and I are constant (no dependency on the $\mathrm{x}$ direction) and the beam itself has no forces acting directly on it reducing q to zero [96].

As previously discussed the base of the sensor is fixed resulting in the boundary conditions Equation $5.2(x=0)$. The free end consists of the concentrated mass and moment resulting from the fluid flow striking the vertical post resulting in boundary conditions, Equation $5.3(x=L)$ [97]. 


$$
\begin{aligned}
y(0, t) & =0 & \frac{\partial y(0, t)}{\partial x} & =0 \\
\frac{\partial^{2} y(L, t)}{\partial x^{2}} & =\frac{M}{E I} & E I \frac{\partial^{3} y(L, t)}{\partial x^{3}} & =m \frac{\partial^{2} y(L, t)}{\partial t^{2}}
\end{aligned}
$$

Here $\mathrm{m}$ is the concentrated mass located and the free end of the beam resulting from the paddle and post. $\mathrm{M}$ is the moment acting on the end resulting from the fluid motion striking the post. The preceding equations require the following assumptions:

1. isotropic materials

2. beam is long and slender

3. deformations remain small

4. plane sections remain plane

5. beam loaded in plane of symmetry

6. vertical post does not deform

7. paddle does not deform

8. negligible drag on beam when deflected

The fluid striking the post generates forces per unit length acting on the post (and thus generating the moment causing beam deflection). These forces consist of the drag force, $F_{d r a g}$, and added mass force, $F_{\text {mass }}$. As they act along the post, the moment is found by integrating along the length of the post (Equation 5.4).

$$
M(t)=\int_{0}^{H}\left(F_{d r a g}+F_{m a s s}\right) y d y
$$

The force of drag acting on a cylinder is given by Equation 5.5 [98]. 


$$
F_{\text {drag }}=0.5 C_{D} D H \rho_{H_{2} \mathrm{O}}\left|U_{\text {rel }}\right| U_{\text {rel }}
$$

where $C_{D}$ is the drag coefficient, $\mathrm{D}$ the post diameter, $\mathrm{H}$ the post height, $\rho_{\mathrm{H}_{2} \mathrm{O}}$ the density of water, and $U_{r e l}$ the velocity of the post relative to the fluid velocity. The drag coefficient for Reynolds numbers $(R e=U L / \nu)$ relevant to this work is found via a correlation provided by White [99]:

$$
C_{D}=1+10 R e^{-2 / 3}
$$

In addition to the drag force, the fluid passing the post generates a force as it accelerates past. The added mass term relates the inertia added to a system caused by the accelerating body moving a volume of surrounding fluid as it moves through it, since the object and fluid cannot occupy the same physical space simultaneously. The added mass per unit length resulting from the oscillating post is [100]:

$$
F_{\text {mass }}=-m \frac{\partial U_{\text {post }}}{\partial t}+\left(m+\rho_{H_{2} O} V_{\text {post }}\right) \frac{\partial U_{H_{2} O}}{\partial t}
$$

where $U_{H_{2} \mathrm{O}}$ is the fluid velocity, $U_{\text {post }}$ the post velocity, and $V_{\text {post }}$ the post volume. For the work presented here the fluid motion is prescribe by:

$$
U_{H_{2} O}=U+u^{\prime} \sin (\omega t)
$$

where $\mathrm{U}$ is the steady state component of the fluid motion, $\mathrm{u}^{\prime}$ the magnitude of the oscillating component of fluid velocity, and $\omega=2 \pi f$ the frequency of the oscillating fluid velocity component. The result is a larger steady flow with an unsteady component. For the work presented here, the steady state fluid flow is $0.1 \mathrm{~m} / \mathrm{s}$ for all results. Table 5.1 list the parameters for the simulations. 


\begin{tabular}{|c|c|}
\hline $\mathrm{U}$ & $0.0,0.1(\mathrm{~m} / \mathrm{s})$ \\
\hline $\mathrm{u}^{\prime}$ & $0.001-.1 \mathrm{~m} / \mathrm{s}$ \\
\hline $\mathrm{E}$ & $170 \mathrm{GPa}[101]$ \\
\hline $\mathrm{H}$ & $550 \mu \mathrm{m}$ \\
\hline $\mathrm{D}$ & $80,160 \mu \mathrm{m}$ \\
\hline $\mathrm{L}$ & $100 \mu \mathrm{m}$ \\
\hline $\mathrm{f}$ & $25-500 \mathrm{~Hz}$ \\
\hline thickness & $1,2 \mu \mathrm{m}$ \\
\hline width & $40 \mu \mathrm{m}$ \\
\hline$\rho_{\text {beam }}$ & $2330 \mathrm{~kg} / \mathrm{m}^{3}[101]$ \\
\hline$\rho_{\text {post }}$ & $1190 \mathrm{~kg} / \mathrm{m}^{3}[102]$ \\
\hline$\rho_{\mathrm{H}_{2} \mathrm{O}}$ & $1000 \mathrm{~kg} / \mathrm{m}^{3}[103]$ \\
\hline$\nu_{\mathrm{H}_{2} \mathrm{O}}$ & $1 \times 10^{-6} \mathrm{~m}^{2} / \mathrm{s}[103]$ \\
\hline
\end{tabular}

Table 5.1: Parameters for haircell sensor modeling

\subsubsection{Finite Difference Solution}

In order to solve the partial differential equation describing the cantilever beam motion (Equation 5.1), a finite difference method is implemented. This is a technique used to solve complex partial differential equations. For brevity, here the explicit second order Runge-Kutta method only is implemented creating a forward difference in time, spatially centered solution [104]. Due to stability issues, an implicit CrankNicolson solution was developed to solve for larger, higher frequency oscillating flows. Both were coded in Matlab and the codes are found in Beam Coding appendix.

The cantilever is divided into $\mathrm{N}$ nodes. The solution starts at the base and moves along the beam, solving for deflection at each node, $\mathrm{N}$, then progressing to the next time step. Below is the governing equation translated into the finite difference scheme:

$$
y_{N}^{t+1}=-\frac{E I(\Delta t)^{2}}{\rho_{\text {beam }} A_{\text {beam }}(\Delta x)^{4}}\left[y_{N+2}^{t}-4 y_{N+1}^{t}+6 y_{N}^{t}-4 y_{N-1}^{t}+y_{N-2}^{t}\right]+2 y_{N}^{t}-y_{N}^{t-1}
$$

The boundary conditions are likewise translated into the finite difference scheme. The fixed end boundary conditions are provided in Equation 5.10 and the free end with the concentrated mass and moment in Equation 5.11. These provide the necessary 
values for $y_{-1}, y_{L+1}$, and $y_{L+2}$ nodes which do not actually exist.

$$
\begin{aligned}
y_{0}^{t} & =0 \\
y_{1}^{t} & =y_{-1}^{t} \\
y_{L+1}^{t} & =2 y_{L}^{t}-y_{L-1}^{t}+\frac{M \Delta x^{2}}{E I} \quad y_{L+2}^{t}=\frac{2 m \Delta x^{3}}{E I \Delta t^{2}}\left(y_{L}^{t+1}-2 y_{L}^{t}+y_{L}^{t-1}\right)+2 y_{L+1}^{t}-2 y_{L-1}^{t}+y_{L-2}^{t}
\end{aligned}
$$

where $\Delta x$ is the distance between nodes and $\Delta t$ is the time step used in the simulation.

The full explicit and implicit codes are included in the appendix. To begin, all the material parameters are defined. The fluid motion is prescribed from Equation 5.8. The number of nodes, time steps, and total time are defined based on the fluid velocity. In each simulation the beam starts at rest. For the first time step the forces from drag and added mass are calculated and the moment determined. This feeds into the finite difference model generating a deflection in the beam at the next time step. This deflection has caused a small displacement in the vertical post. The resulting change in position of the post is assumed to match the angle of the final node of the cantilever beam. Thus as the free end of the beam angles downward at 0.01 rad, the post is now angled by that same amount, and the vertical post has velocity and acceleration. This is taken into account in the moment via the added mass and drag force equations in the terms for relative velocity and post acceleration. In this manner a simple fluid structure interaction is conducted. To avoid suddenly striking the beam with a large force, the code slowly ramps up the resulting moment over the first quarter of the simulation. The initial limiting of fluid forces had no bearing on the steady state results obtained over the entire solution. In the explicit model the time step was required to be small to insure stability. Due to the large computational times required at higher frequencies and velocity magnitudes, a secondary implicit code was created. Both generated matching results and were verified analytically for a simple constant force deflection. 


\subsection{Results}

The haircell sensor model was first subjected to a $0.1 \mathrm{~m} / \mathrm{s}$ steady flow with an oscillating component magnitude of $0.025 \mathrm{~m} / \mathrm{s}\left(u^{\prime} / U=0.25\right)$. The frequency of the oscillating component of the fluid velocity ranged from 25-250 Hz. For the results presented here the length, width, and thickness of the beam were $100 \mu \mathrm{m}, 40 \mu \mathrm{m}$, and $2 \mu \mathrm{m}$ respectively. The post was $550 \mu \mathrm{m}$ tall with a diameter of $80 \mu \mathrm{m}$. These sensor dimensions are referred to as the base parameters. For all cases the beam was divided into 30 nodes $(\mathrm{N})$.

The first important feature to note results from the change of the moment applied to the end of the cantilever as the oscillatory fluid frequency increases. Figure 5.3 shows the fluid motion, force due to drag, force from the added mass, and the resultant moment for the fluid flow with a $25 \mathrm{~Hz}$ component. Here the moment is dominated by the drag force which is two orders of magnitude higher than that of the added mass. As a result the moment closely matches the oscillation of the fluid.

As the oscillation frequency increases to $250 \mathrm{~Hz}$, the force due to added mass begins to play a larger roll in the moment acting on the sensor. Figure 5.4 shows the same force and moment parameters previously but now the added mass is just less than one order of magnitude smaller than that of the drag force (which remains the same). The added mass force is a result of the acceleration of the fluid around the post. For this reason it has the same oscillating frequency as that of the fluid motion and drag forces but is offset by 90 degrees. The force from added mass peaks at the location of the largest slope of the fluid velocity plot. Therefore the peaks of the moment acting on the cantilever occur just prior to the actual peaks in the fluid motion and are no longer a simple sine wave. In both situations the total force acting on the post is in the $\mu \mathrm{N}$ range.

The resulting tip deflection as a function of time is plotted in Figure 5.5 for the $0.1 \mathrm{~m} / \mathrm{s}$ flow with a $250 \mathrm{~Hz}$ oscillatory component. Note the transverse direction (y) is downward resulting in positive cantilever tip deflections and the force is gradually 

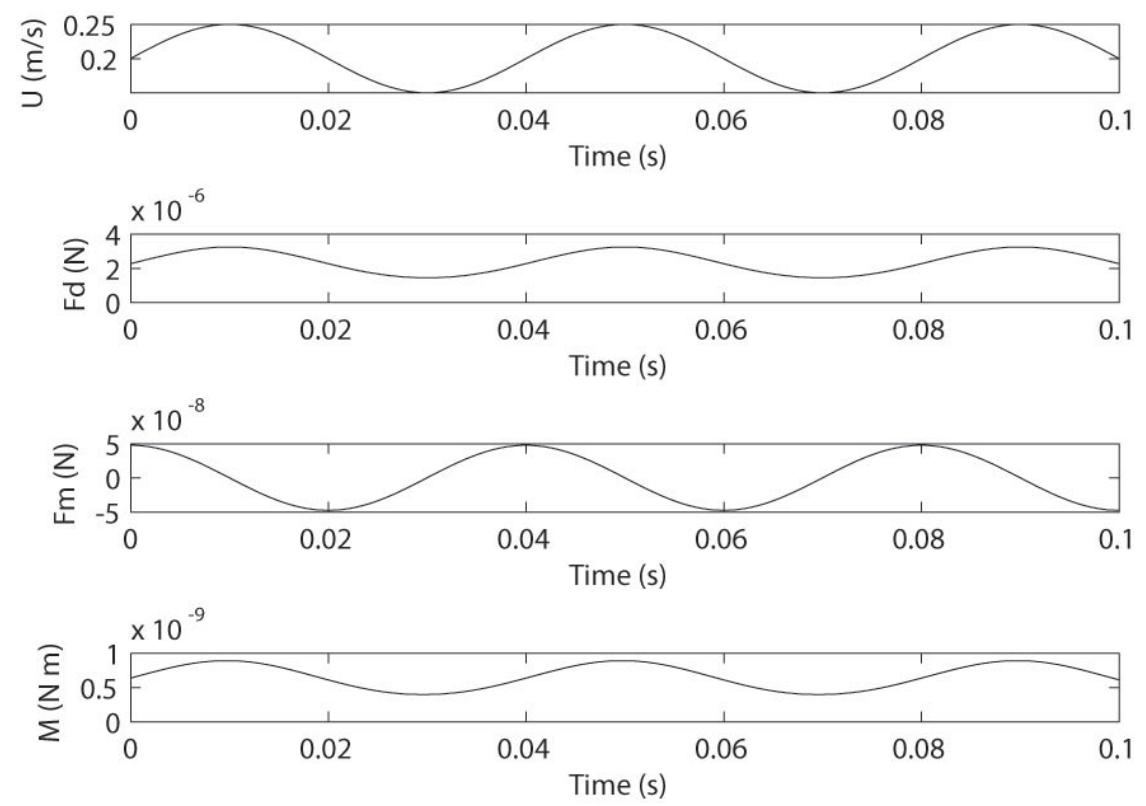

Figure 5.3: Resulting drag dominated moment from a fluid motion of $25 \mathrm{~Hz}$.
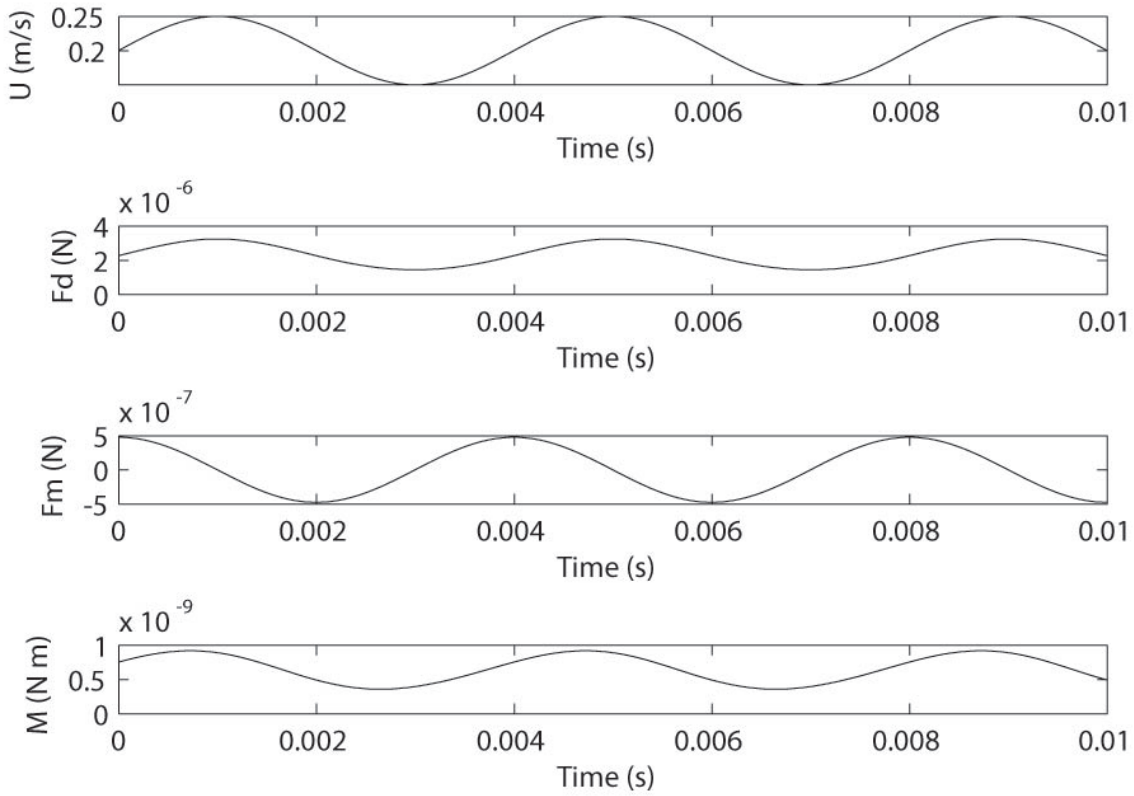

Figure 5.4: Resulting added mass influenced moment from a fluid motion of $250 \mathrm{~Hz}$. 

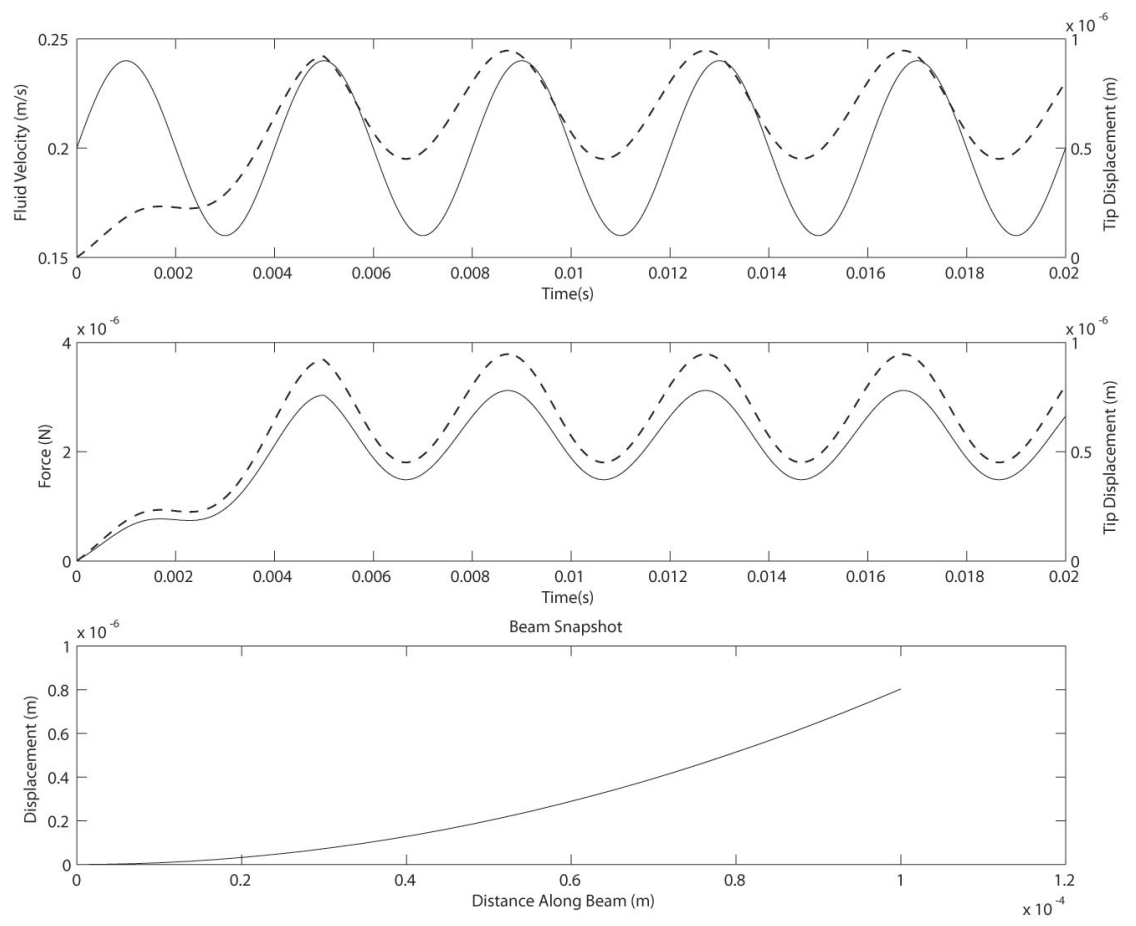

Figure 5.5: Dynamic results for the $250 \mathrm{~Hz}$ simulation. The dashed line represents the cantilever tip displacement. The bottom plot shows the cantilever at its maximum deflection (magnitude).

built up over one cycle of fluid motion for stability. The top plot shows the fluid velocity (solid line) and the tip displacement (dashed line). Since the force from added mass is comparable to that of the drag, the maximum tip displacements occur just before the peaks and troughs of fluid motion. The middle plot shows the total force acting on the post (solid line) and tip displacement (dashed line). The repeated motion of the tip displacement shows steady state is reached. The cantilever beam is modeled as an elastic material so it follows the force as expected. The bottom plot illustrates a snap shot of the entire length of the sensor at its maximum deflection (plotting the location of all 30 nodes). The maximum tip deflection is on the order of $1 \mu \mathrm{m}$ and therefore the assumptions outlined earlier are not violated.

Using the same base parameters for the sensor dimensions, Figure 5.6 shows the influence of the oscillating component of the fluid velocity. The magnitude of the 


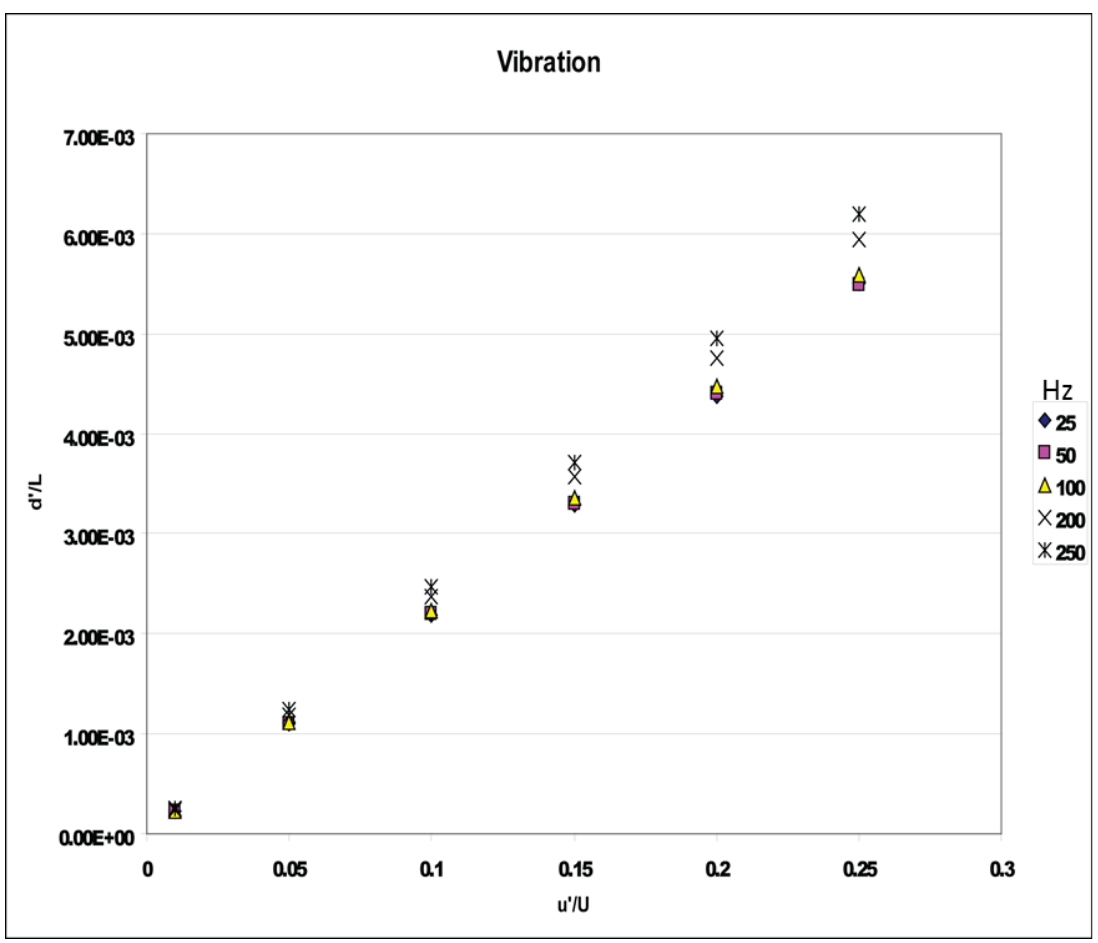

Figure 5.6: Maximum haircell tip deflections for the base parameters for frequencies from 25 to $250 \mathrm{~Hz}$ and $u^{\prime} / U$ from 0.01 to 0.25 .

oscillating velocity component, u', ranges from 0.001 to $0.025 \mathrm{~m} / \mathrm{s}$ and its frequency ranges from 25 to $250 \mathrm{~Hz}$. At small oscillating magnitudes there is very little difference between all the frequencies. Likewise there is little difference between frequencies of 25-100 Hz for all velocity magnitudes. This is a direct result of the force from added mass playing little role in the moment placed on the beam. Of greater importance is the linear trend as the oscillating magnitude increases for a given frequency. A nonlinear trend is observed for increasing frequency at a given oscillating magnitude.

Figures 5.7 and 5.8 illustrate the resulting maximum tip deflections for doubling the diameter of the post to $160 \mu \mathrm{m}$ and the halving the beam thickness to $1 \mu \mathrm{m}$. The same linear and non-linear trends that were described previously remain. In Figure 5.7 the maximum tip deflections have about doubled from those of the base parameters. The non-linear trend with increasing frequency is more evident. The forces and resultant moment on the sensor are much larger due to the increase in post size. Due to the increase in post volume the added mass force plays a significant 


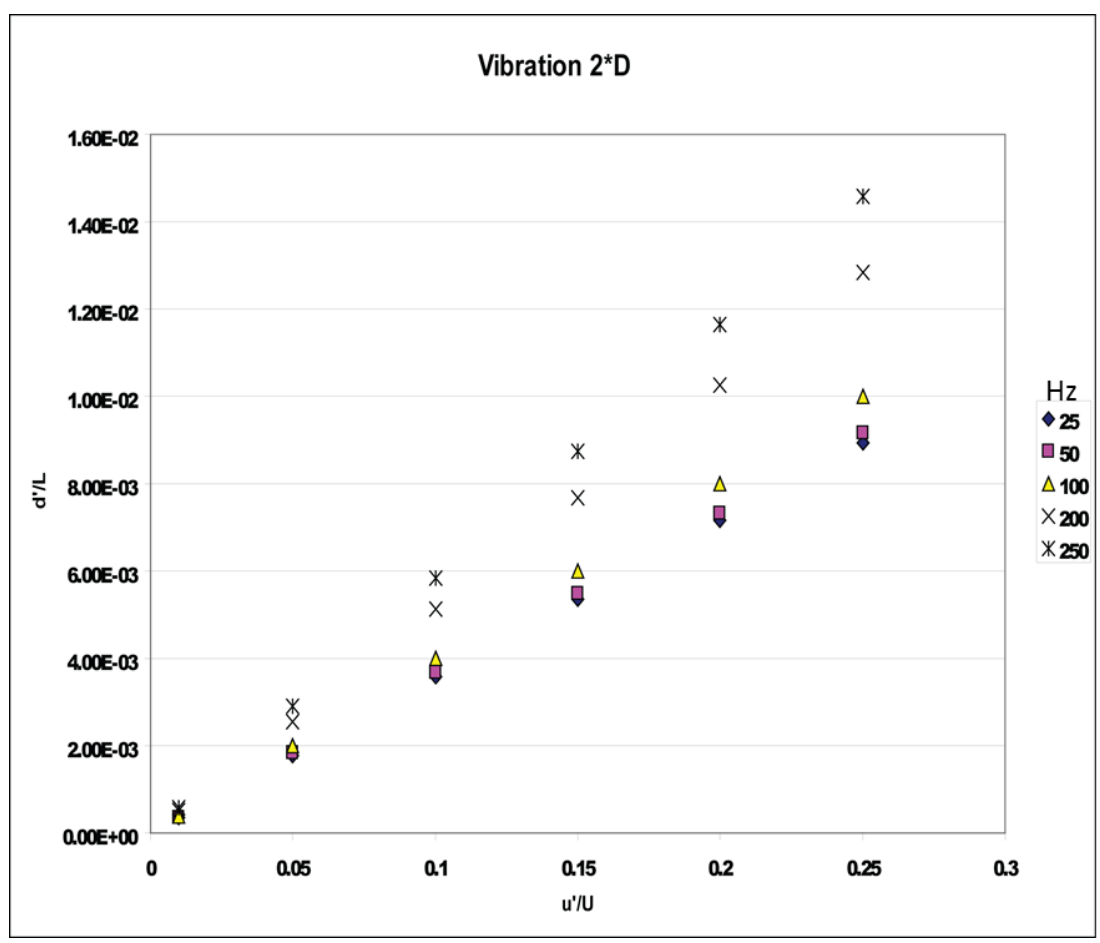

Figure 5.7: Maximum haircell tip deflections for doubling the diameter for frequencies from 25 to $250 \mathrm{~Hz}$ and $u^{\prime} / U$ from 0.01 to 0.25 .

role as the oscillating frequency increases. The fluid must accelerate more to move around the post. In Figure 5.8 the result trends closely match that of Figure 5.6. However the maximum deflections have increased by an order of magnitude. The sensor is much more sensitive to fluid motion.

Figure 5.9 illustrates how the change in beam thickness and post diameter influence the sensor's maximum responses. The results here are shown for the oscillating flow magnitude of $0.025 \mathrm{~m} / \mathrm{s}$. The modification to the cantilever thickness has the greatest improvement to the maximum deflections. This comes at a cost of weakening the main support of the haircell sensor. The increase in post diameter likewise increases the sensitivity. However, in doing so the mass acting on the cantilever tip increases resulting in negative effects examined in the next simulations and interpreted in the discussion.

Reverting back to the sensor base parameters, simulations modeling the sensor response to an oscillating flow only were performed. The fluid velocity oscillated with 


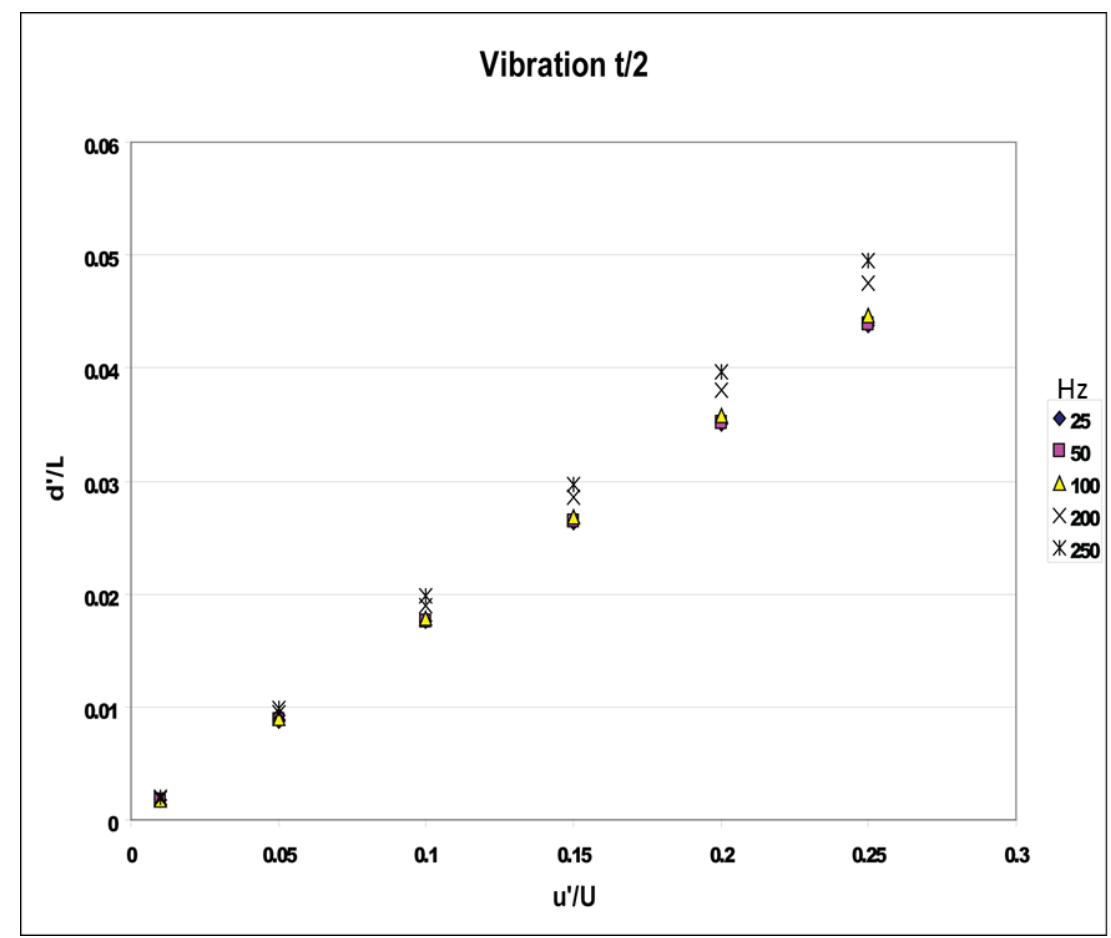

Figure 5.8: Maximum haircell tip deflections for halving the thickness for frequencies from 25 to $250 \mathrm{~Hz}$ and $u^{\prime} / U$ from 0.01 to 0.25 .

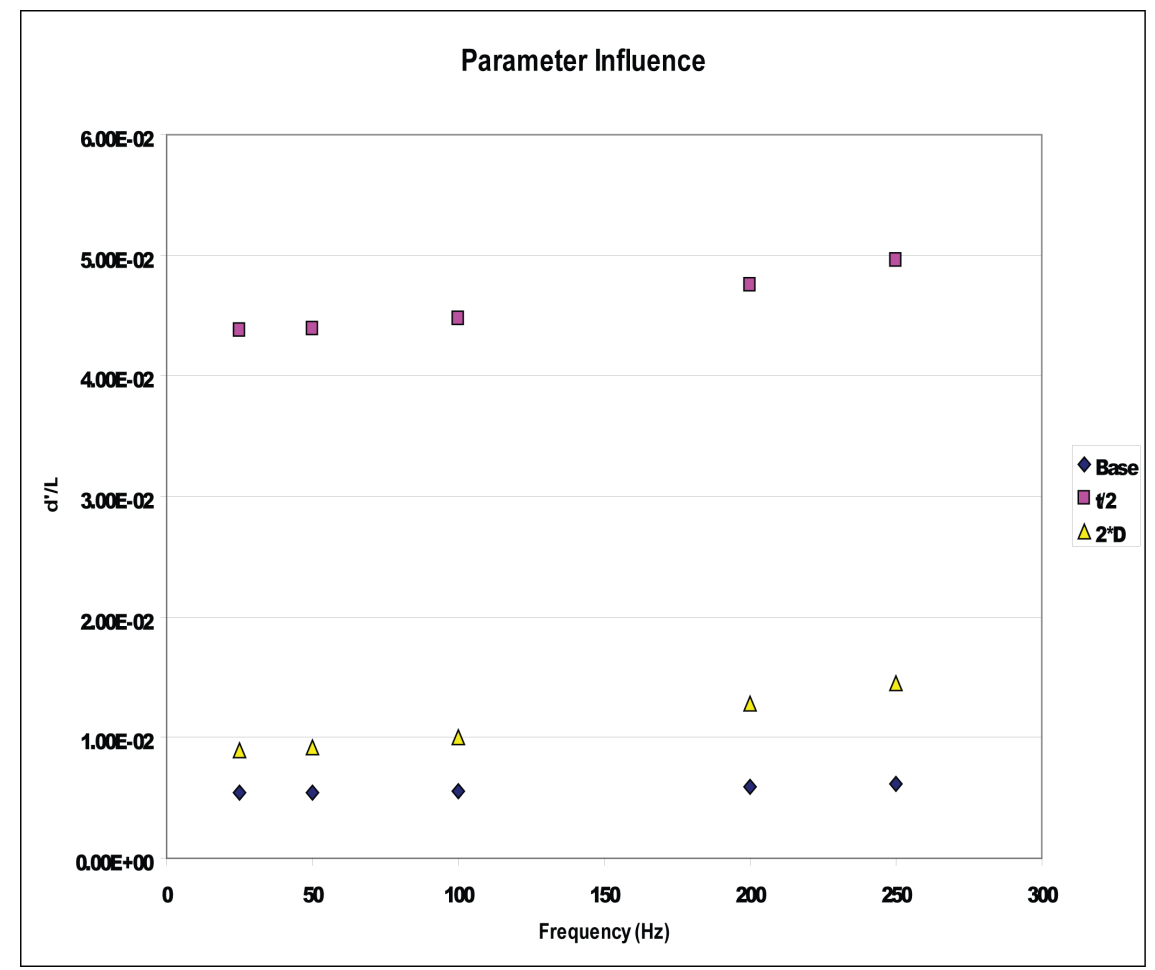

Figure 5.9: Maximum haircell tip deflections vs frequency showing design changes, $u^{\prime} / U=.25$. 

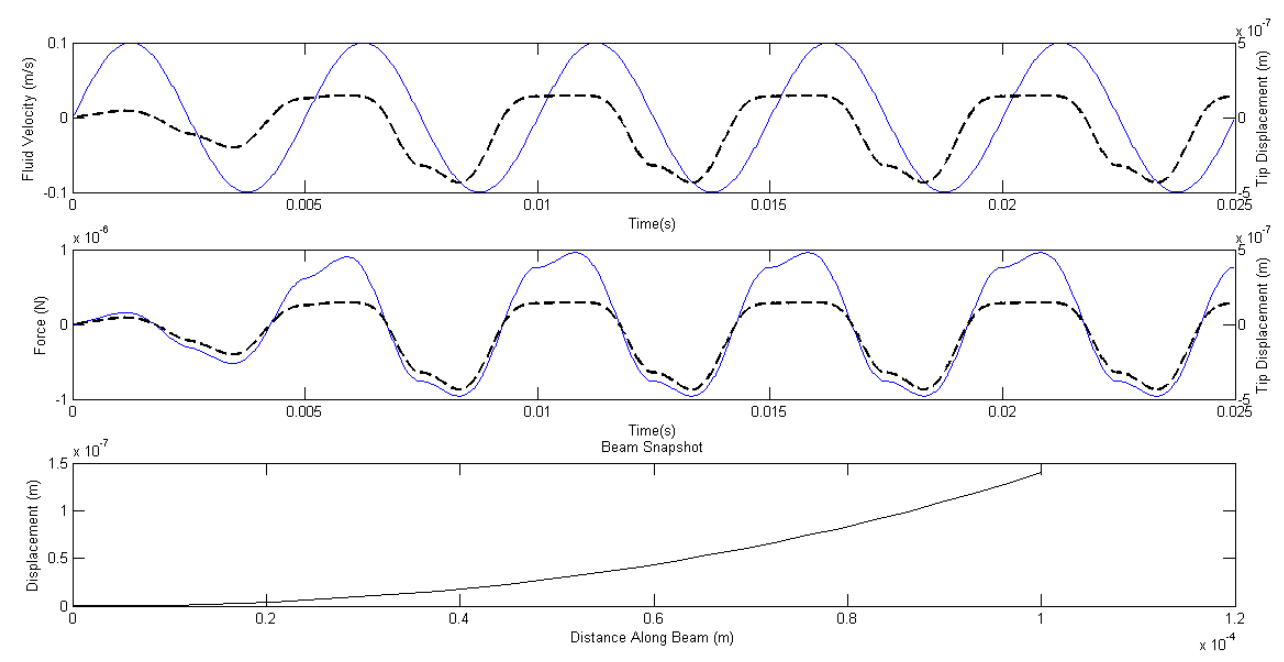

Figure 5.10: Haircell sensor response to an oscillating flow with $0.1 \mathrm{~m} / \mathrm{s}$ flow magnitude oscillating at $200 \mathrm{~Hz}$. The dashed line represents the cantilever tip displacement. The bottom plot shows the cantilever at its maximum deflection (magnitude).

a magnitude of $0.1 \mathrm{~m} / \mathrm{s}$ and frequencies ranged from 1-500 Hz. In Figure 5.10 the top plot shows the fluid velocity (solid line) and the tip displacement (dashed line). The middle plot shows the total force acting on the post (solid line) and tip displacement (dashed line). The repeated motion of the tip displacement shows steady state is reached. The role of added mass is evident in the force plot. The bottom plot illustrates a snap shot of the entire length of the sensor at its maximum deflection (plotting the location of all 30 nodes). The maximum tip deflection is on the order of $0.14 \mu \mathrm{m}$.

The key difference between the previous simulations with a steady flow much larger than the oscillating component is that the tip deflection is capped in one direction. Here it is shown that the sensor has a significant preference for a single direction of motion. This is caused by the lumped mass acting on the end of the cantilever. When the fluid motion is in the direction causing the beam to bend downward it closely matches the force applied to the post. However, when the fluid motion causes the beam to deflect upward the mass limits the motion. This phenomena was not observed in the previous case as the steady state flow component always forced the 


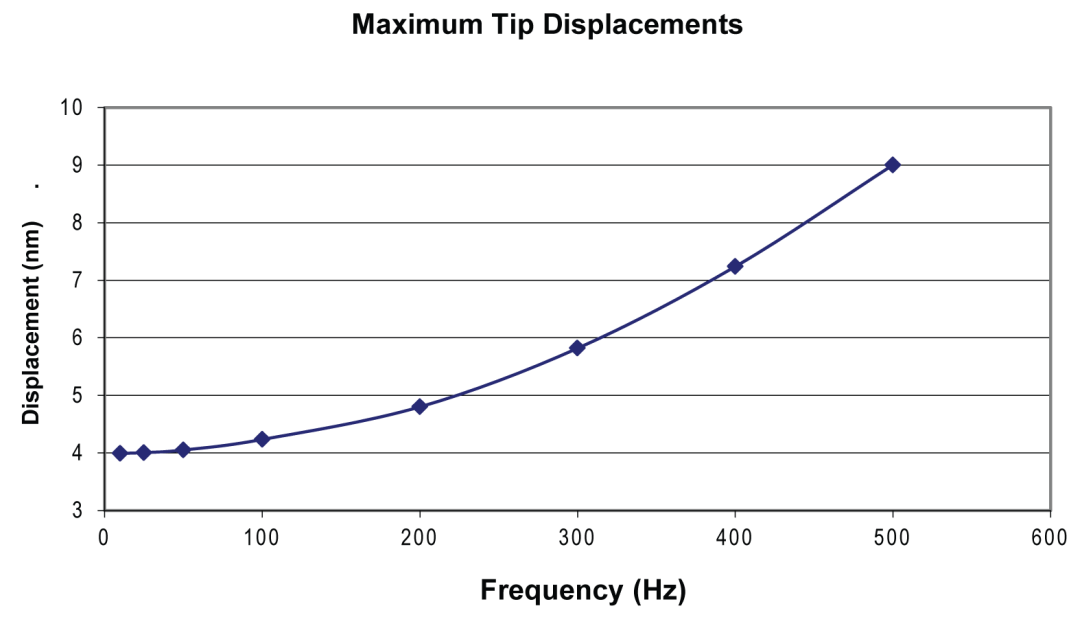

Figure 5.11: Haircell sensor response vs frequency to an oscillating flow of amplitude $0.1 \mathrm{~m} / \mathrm{s}$.

beam downward where it then oscillated. In this case the the mass caps the maximum upward beam deflection. This implies the sensor is limited to not just a single axis of fluid motion but also only to a single direction along that axis. The haircell sensor only accurately captures fluid motion in the direction of the base to the post. Therefore to capture the 2D flow field four haircell sensors are required each at a 90 degree offset.

The non-linear effect of increasing frequency was noted in previous simulations. Here using only the simple oscillating motion the frequency was varied from 1-500 Hz. Figure 5.11 shows the trend of the maximum beam tip deflection as the frequency of fluid motion increases. The beam tip displacement increases in a quadratic fashion as the frequency increases.

\subsection{Validation}

Validation of the simulations were made through experimental tests of the haircell sensor under the same base parameters by Chen [8]. In these experiments a dipole of a shaking sphere was placed in a tank with the haircell sensor. The sphere was $6.35 \mathrm{~mm}$ in diameter and located $15 \mathrm{~cm}$ above the sensor. The sphere vibrates along the axis 


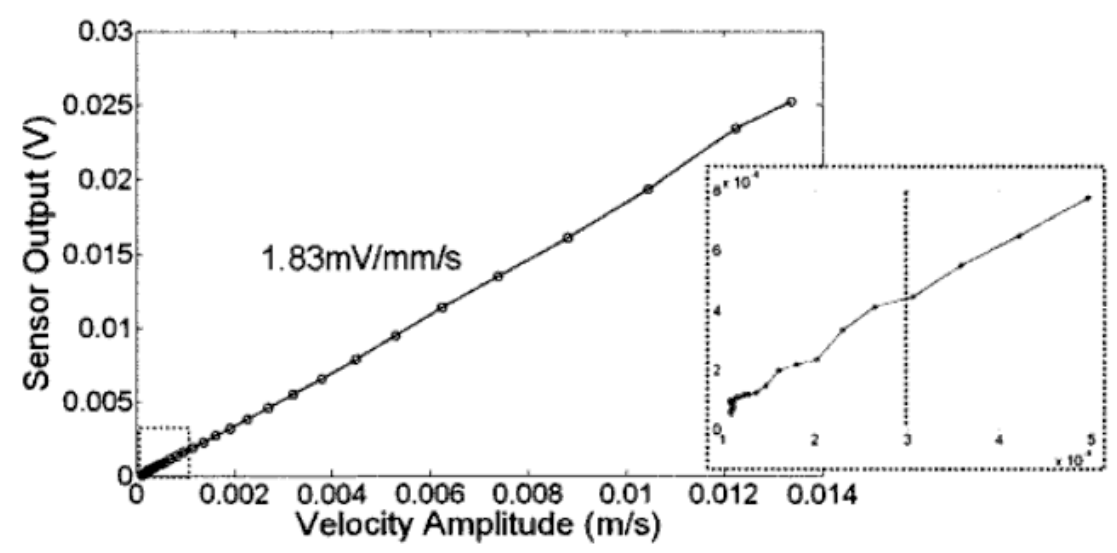

Figure 5.12: Haircell sensor response to an oscillating flow [8].

of the sensor and is controlled by a shaker dictating the amplitude and frequency of the sphere motion. This generates an oscillating fluid flow along the sensor deflection axis.

Figure 5.12 shows the sensor output as the fluid velocity amplitude increases from a dipole oscillating flow of $50 \mathrm{~Hz}$. The trend is linear and matches the linear trend observed in the first set of simulations where the tip deflection increased linearly at a given frequency as the oscillating fluid component increased. Figure 5.13 shows the sensor response as the frequency is increased for an oscillating flow amplitude of 1 $\mathrm{mm} / \mathrm{s}$. The trend is not clearly quadratic, likely due to the small range of frequencies tested, but the sensor output increases with the frequency as observed in Figure 5.11. The simulations match the trends observed in the experiments.

\subsection{Discussion}

The haircell sensors exhibit potential high sensitivity and are capable of capturing information about the flow field. However the deflections of the sensor are very small. Increasing the sensor deflection or sensitivity comes at costs. Increasing the post size allows the haircell to capture the fluid forces better, however the increase in mass limits the actual improvement. As the mass increases the sensors become more 


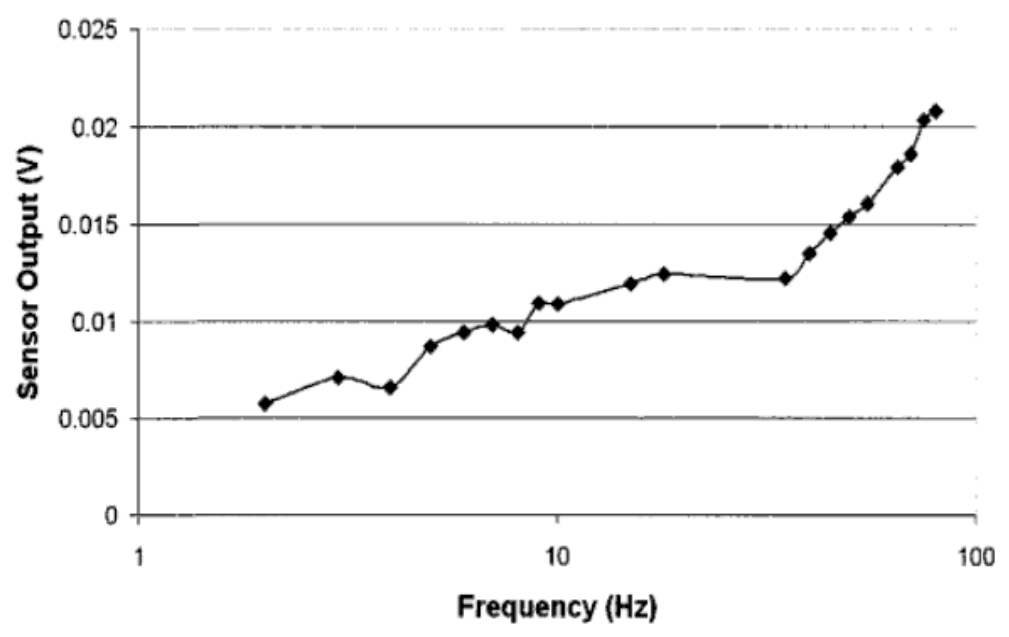

Figure 5.13: Haircell sensor response to an oscillating flow [8].

limited to detecting fluid motion only in the direction aligned with the cantilever base to the post. It would require multiple sensors in different orientations to correctly capture fluid motion.

The greatest increase to sensitivity occurs by shrinking the cantilever beam thickness. This comes at a cost of the structural integrity of the haircell sensor. The sensors in the base parameter design suffered reliability issues from damage. Shipping several arrays of the haircell from Illinois to Virginia resulted in nearly a $90 \%$ failure rate despite careful packaging. In shipping the haircells remained untouched but the package jostling was enough to permanently deform the cantilevers. If the post was struck by a solid object the sensor failed. Due to the small size repairs are impossible.

The smallness of the sensor also limits their useful deployment. They prove to have great response to small oscillations in an otherwise still environment (similar to that of a spider trichobothria). However once placed on a moving surface they are potentially immersed in the object's boundary layer. If the sensors are embedded in boundary layers developing over the support surface, and if the flow in this boundary layer is transitional or turbulent, there may be considerable distortion of the original hydrodynamic signal [105]. It is unknown how sensor performance is impacted by 
this added noise. Thus the haircell sensors are ill-suited for tracking wakes where a vehicle must chase another.

The contributions of this chapter include the development of a fluid-structure interaction code to capture the kinematics of a cantilever haircell sensor. The impact of the fluid flow forces on a post with respect to oscillatory frequency are examined. The effect of the design parameters cantilever thickness and post diameter are quantified. The limitations of this type sensor are outlined.

Due to the limitations of sensor frailty, unknown response when immersed in a boundary layer, and ability to monitor fluid motion in only one direction, the research here progresses to a new sensor design inspired by pinniped vibrissae. These sensors will protrude well beyond the boundary layer to capture the flow hydrodynamics present away from the surface. The next chapter describes this sensor development.

\subsection{Summary}

The goal of this chapter was to model the haircell sensor, inspired by fish neuromasts and spider trichobothria, response to fluid flows. Reviewing the research questions presented at the start of the chapter, the cantilever thickness had the highest impact on the haircell's sensitivity to fluid flows. The sensor was capable of monitoring steady flows and oscillating flows up to at least $500 \mathrm{~Hz}$ as modeled with small deflections. The mass of the post on the end limited the sensor response to fully capturing fluid forces in one direction and the small size scale limited its durability and potential placement on moving bodies. 


\section{Chapter 6}

\section{Bio-Whisker Sensor Development}

Owing to the limitations of the haircell sensor design examined as discussed in Chapter 5 and the incredible ability of pinnipeds to detect small fluid motions and track submerged objects with only the sensory information available from their vibrissae, a new sensor design is proposed and developed in this chapter utilizing several key features of the spider trichobothria and pinniped vibrissae and capable of monitoring flow in two directions with a single sensor. The fluid motion sensor examined here is hybrid in concept, meaning that it combines desirable geometrical and dynamical features present in seal whiskers (such as their length to diameter ratio and inertial

flow operation range) with desirable structural features present in the membrane-like support of a spider wind sensing filiform hair (such as the damping constant and torsional restoring constant of the hair support).

The major contribution of this chapter is the development and testing of the bioinspired whisker-like sensor, which ties into the thesis statement by using knowledge developed from the study of spider and pinniped fluid motion sensors to dictate the design parameters. Research questions answered in this chapter include:

- What design is best suited for incorporation into a compact array?

- Can the sensor survive for extended periods of time in an aquatic environment? 
- Can the sensor detect fluid motion in two directions?

- Are the measurements repeatable?

- Can the sensor capture wake information?

- Can the sensor survive large forces or impacts?

To answer these questions several sensor designs are discussed and the development of a selected model moves forward through four revisions (one as a reference and three by the author). Each revision is tested and evaluated analytically to find design faults and improve the senor's capabilities and sensitivity. Success is ultimately measured by the sensor to detect the presence of a wake and correctly capture a wake hydrodynamic signal in a similar fashion to that observed in the vibrissae study.

\subsection{Whisker-like Sensor Designs}

The bio-inspired sensor design is motivated by the spider hairs examined in Chapter 3 and the pinniped vibrissae tested in Chapter 4 . The goal is to combine features of both systems into a hybrid sensor for use in water. A key design feature of trichobothria is the flexible membrane supporting the hair which provides the damping and restoring effects to the hair motion. A key feature of the seal whiskers is their projection into the fluid stream to remove unwanted information in the fluid motion near the surface. This is an important feature differentiating the small spider hairs designed to detect fluid motion from nearly still air and pinniped vibrissae designed to collect flow information while swimming in water. The membrane of the spider trichobothria and the vibrissae consist of relatively soft materials compared to those used in prior bio-inspired sensors such as the MEMS haircell sensor. For this reason the whisker will be supported by polydimethylsiloxane (PDMS) a soft inert compound. 

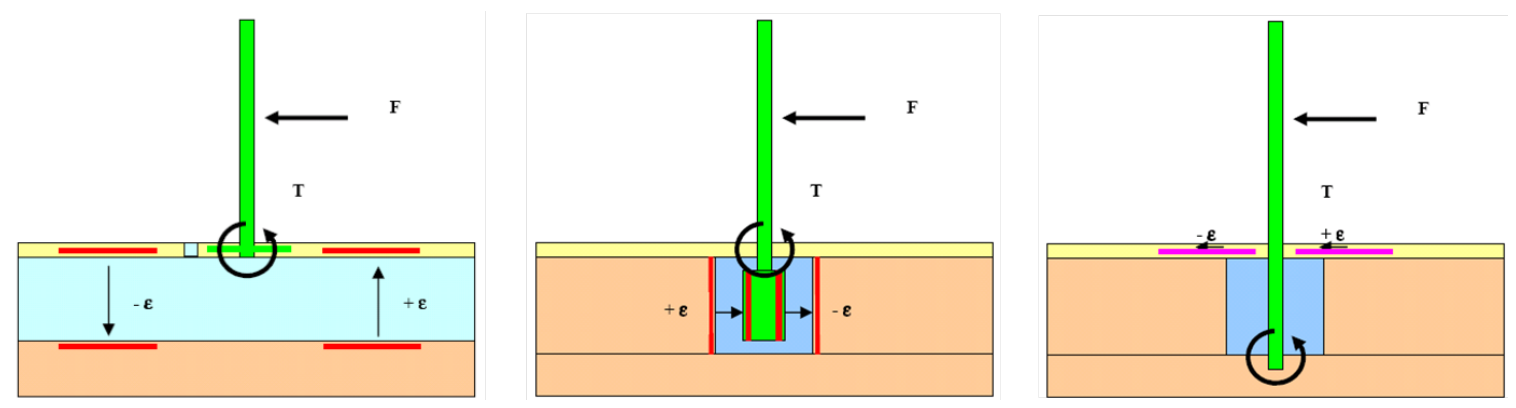

Figure 6.1: Basic sensor design ideas: Capacitor measurement sensors of Barbier el al [9] (left) and modified capacitance sensor (middle) and strain measurement sensor (right).

Prior work by Barbier et al [9] (discussed in Chapter 2) demonstrated the ability of rigid whisker-like sensors to measure deflection through capacitance. The Barbier design is shown in Figure 6.1 on the left. Two circular thin parallel copper plates (red lines) surround the vertical post allowing for motion detection in two orthogonal directions. One of the plate pairs rests below the sensor while the other resides within the PDMS membrane supporting the post/whisker. Fluid forces, F, striking the post generate a a torque, $\mathrm{T}$, at the post base embedded in a thin PDMS membrane. This causes the PDMS membrane to deflect a small distance vertically, $\epsilon$, thus changing the relative capacitance between the plates. The gap between the parallel plates is allowed to fill with the surrounding fluid from the environment and the plates are insulated via the materials in which they reside.

A variation of this design is shown in the middle schematic of Figure 6.1. Again the pivot point of the post is located at the thin PDMS membrane. In this case the parallel capacitance plates are wrapped cylindrically around the cylindrical base of the post and the outer wall (again in four quadrants to provide directional information). However the space between the plates is filled with a custom PDMS material providing the restoring and damping characteristics.

A third variation in design moves the pivot point and location of the torque to the bottom of the cylindrical enclosure. The sensor kinematics are again provided by a thin PDMS membrane, however the post deflection is measured by four strain 


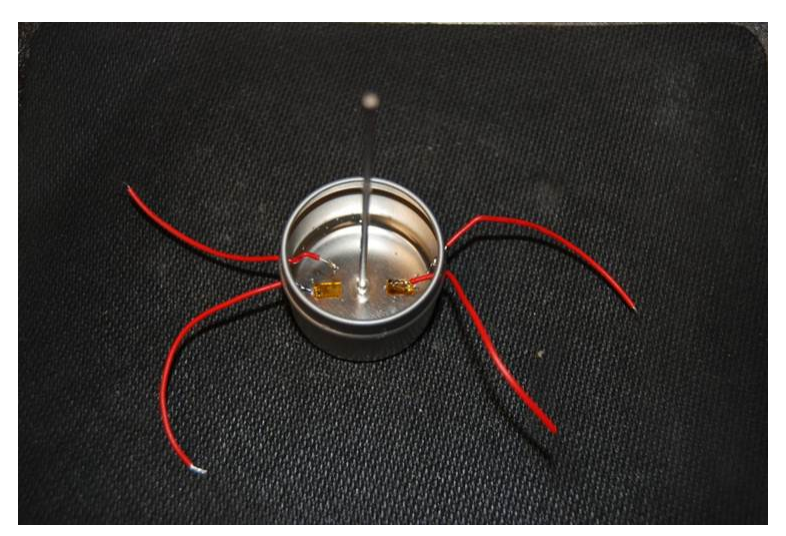

Figure 6.2: Whisker-like sensor with strain gauges embedded in PDMS.

gauges embedded in the PDMS.

Testing of the first design was documented in Chapter 2 and is not reviewed again here. The design showed high sensitivity to post deflections however the connection between the post and PDMS membrane was very delicate. The sensor could be damaged if struck by large forces and was not repairable. Any forces pressing downward on the membrane likewise cause false motion measurements.

Testing of the feasibility of the other two designs was conducted through a simple prototype shown in Figure 6.2. A vertical post of diameter $2 \mathrm{~mm}$ and length $6 \mathrm{~cm}$ was attached to the base of a hollow aluminum cylinder. PDMS was poured into the canister to $1 \mathrm{~cm}$ depth. Two piezoresistive strain gauges were placed on this PDMS layer followed by another layer of PDMS effectively embedding the strain resistors within the PDMS. This created a whisker pivoting at the base as shown in the third design, but with PDMS surrounding the whisker as shown in the second schematic. Forces applied to the post generated very small deflections of the post, none of which were captured by the strain gauges. Two observations were made. The stain gauges were unable to fully bond to the PDMS and as a result any deformation of the material essentially slid over the gauge. The forces required to generate deflections were much higher than those expected in a fluid flow (such as those described in the previous chapter). The high viscosity of PDMS severely limited to motion of the post. However the sensor itself was robust and experienced zero failures under high loads 


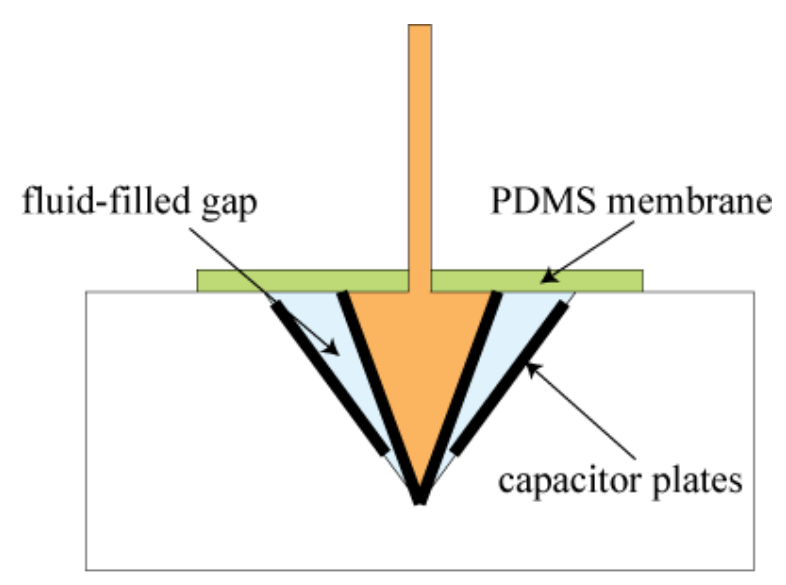

SIDE VIEW

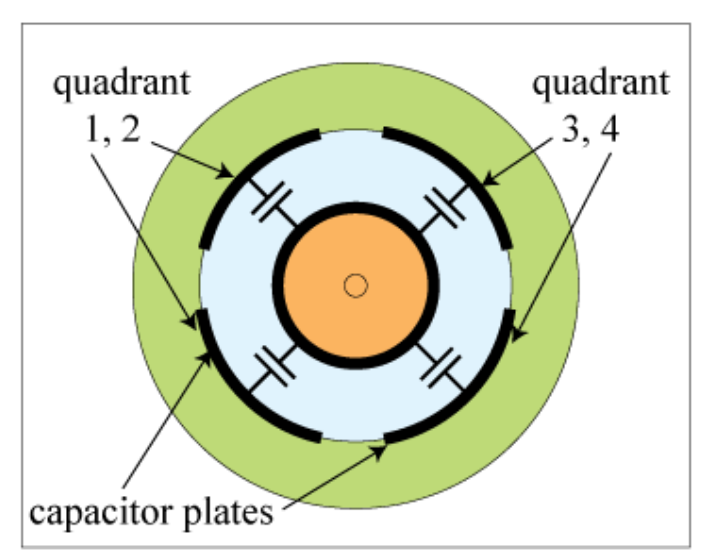

TOP VIEW

Figure 6.3: Side and top view schematics of whisker-like cone-in-cone sensor design.

or suddenly imposed forces.

These results led to the conclusion the sensor design needed the sensitivity provided by the Barbier sensor and strength of the tested strain sensor. The post needed to pivot about the base but the restoring forces needed to come solely from a thin PDMS membrane along the top of the sensor. This led to a variation of the second design with consideration for generating a circular array. The hybrid whisker design incorporates a cone-in-cone design illustrated in Figure 6.3.

The new design base structure allows the whisker to firmly embed in a cone-incone design (pivoting at the cone tip), in which the sides of the cones facing each other are metallically plated and sealed with epoxy to create a parallel plate capacitor. The 
plates on the outer cone are divided into four quadrants in order to provide directional information of the flow. Considering the dimensional features of trichobothria and vibrissae, in particular the length-to-diameter (L/d) ratios ranging from 10-100 [85] and a characteristic length of seal whiskers ranging from 2-10 $\mathrm{cm}$ [61, the sensor post was selected to be $4 \mathrm{~cm}$ in length and $2 \mathrm{~mm}$ in diameter, yielding an $\mathrm{L} / \mathrm{d}$ ratio of 20. Although these dimensions are on the small end of the spectrum, this size allows for easy fabrication. The microtrichs found on the spider hairs and the undulated flattened features of harbor seal vibrissae features are also currently missing from the post to aid in fabrication and reduce complexity of capturing the sensor's response to fluid flows. In order to provide an appropriate damping and restoring force, the cone-in-cone base is covered by a thin membrane of custom-fabricated PDMS, approximately $200 \mu \mathrm{m}$ in thickness. This overall design maximizes the surface area of the capacitor plates to increase signal magnitude, especially when multiple sensors are arrayed around a cylindrical vessel, as well as provides for a more durable sensor construction.

\subsection{Sensor Fabrication}

Fabrication of the sensor structural components is done by transfer molding of epoxy through flexible silicon molds. In the case of the base and the cone structures, the masters are created first in a computer solid model which is then produced by stereo lithography. The pie wedge shaped conductive pads on the base are etched in-house at Mikro Systems, and pressed into the cone-base with a molded rubber form. The movable cone is covered in another conductive surface. Wires are attached with the silver epoxy to the 5 capacitive surfaces. The whisker post which transmits the hydraulic force into the sensor is attached into a hole that is pre-cast in the movable positive conical part. Both components are then water-proofed by coating with the same epoxy in which they are cast. They are then assembled together with 


\begin{tabular}{|c|c|}
\hline Geometry & Measurement \\
\hline whisker height & $40 \mathrm{~mm}$ \\
\hline whisker diameter & $2 \mathrm{~mm}$ \\
\hline inner cone height & $18 \mathrm{~mm}$ \\
\hline inner cone diameter & $23 \mathrm{~mm}$ \\
\hline outer cone height & $18 \mathrm{~mm}$ \\
\hline outer cone diameter & $26 \mathrm{~mm}$ \\
\hline gap between cones & $1.5 \mathrm{~mm}$ \\
\hline PDMS thickness & $0.2 \mathrm{~mm}$ \\
\hline
\end{tabular}

Table 6.1: Dimensions of prototype whisker-like sensor

a thin, very flexible membrane which is blended at Mikro Systems from 3 different elastomeric resins. The thickness and durometer were matched to the needs of this application.

\subsection{Sensor Prototype}

The author of this work played a key role on the ideas and designs leading to the first whisker-like sensor prototype and conducted all the design improvements and testing following the first revision. Tests conducted on the first revision were conducted by Stocking in his Master's work [11] and highlights of his work are presented here to illustrate the design's capabilities and demonstrate how the author then improved the sensor development.

Figure 6.4 shows the the first sensor prototype. The capacitance plates on the outer cone consist of $0.05 \mathrm{~mm}$ copper plates. The inner cone is painted with a layer of silver filled conductive epoxy. As previously mentioned both surfaces are protected by a layer of epoxy. The gap between the cones is filled with an inert liquid, silicone oil. Capacitance measurements across the plates were made with a B and K Precision model 890B meter. The prototype sensor dimensions are provided in Table 6.1 .

Force deflection measurements for each test were conducted by Stocking for each quadrant of capacitor plates. The tests were conducted in a similar fashion to those described in Chapter 4 with the capacitance meter capturing the sensor output. Fig- 


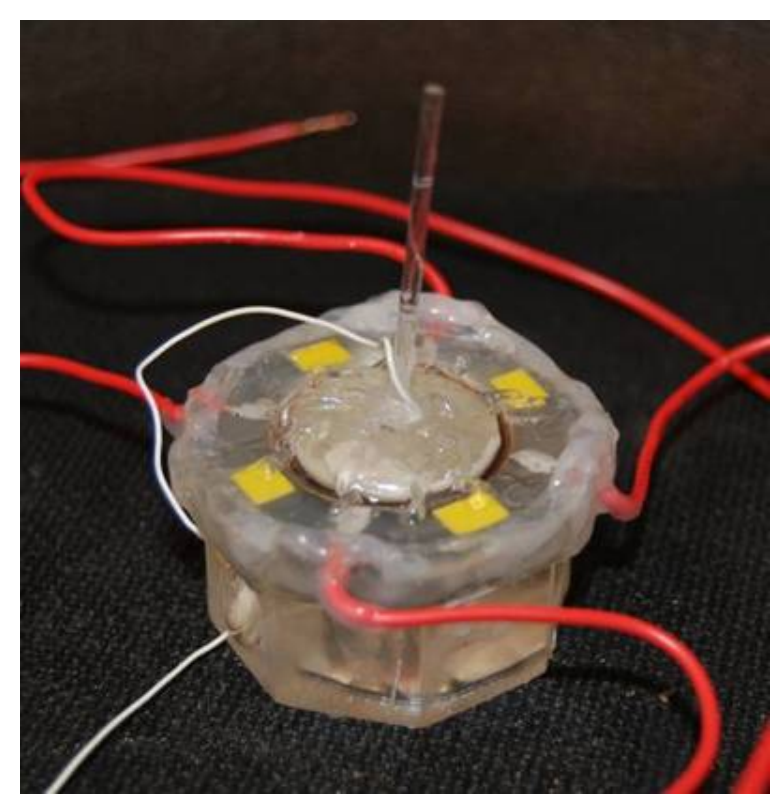

Figure 6.4: First whisker-like sensor prototype.

ure 6.5 shows the capacitance of each quadrant under compression as the gap between the plates shrinks. Figure 6.6 shows the capacitance change as force is applied to the post causing the plates to separate and the gap to grow. Forces were capped at 30 $\mathrm{mN}$ matching the drag force on the whisker from a $1 \mathrm{~m} / \mathrm{s}$ water flow. The results are averaged over five trials and the standard deviation is within the data point markers.

Three of the quadrants in compression measured very similar changes of roughly $3 \mathrm{pF}$ from minimum to maximum load, while one quadrant showed nearly an $8 \mathrm{pF}$ change. In separation the changes were much less pronounced, typically only measuring a $0.5 \mathrm{pF}$ change from minimum to maximum; although, one quadrant did register a nearly $2 \mathrm{pF}$ change.

Placing the prototype sensor in the water flume attached to the sting in the same manner described in Section 4.3.2, the sensor was aligned placing quadrant three facing the flow. Thus as the water speed increased, forces on the post should increase the compression on quadrant three and therefore increase the capacitance reading. Figure 6.7 shows the results. The sensor does register an increase in capacitance as the flow speed increases, however the standard deviations eclipsed the capacitance 


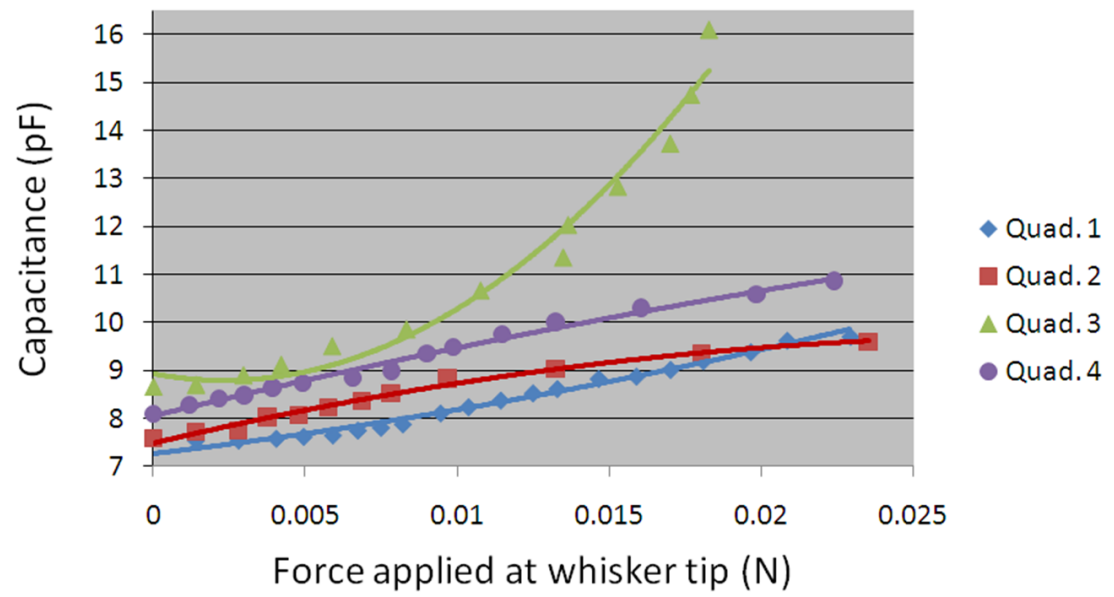

Figure 6.5: Force-capacitance measurement of each quadrant in the prototype sensor in compression [10].

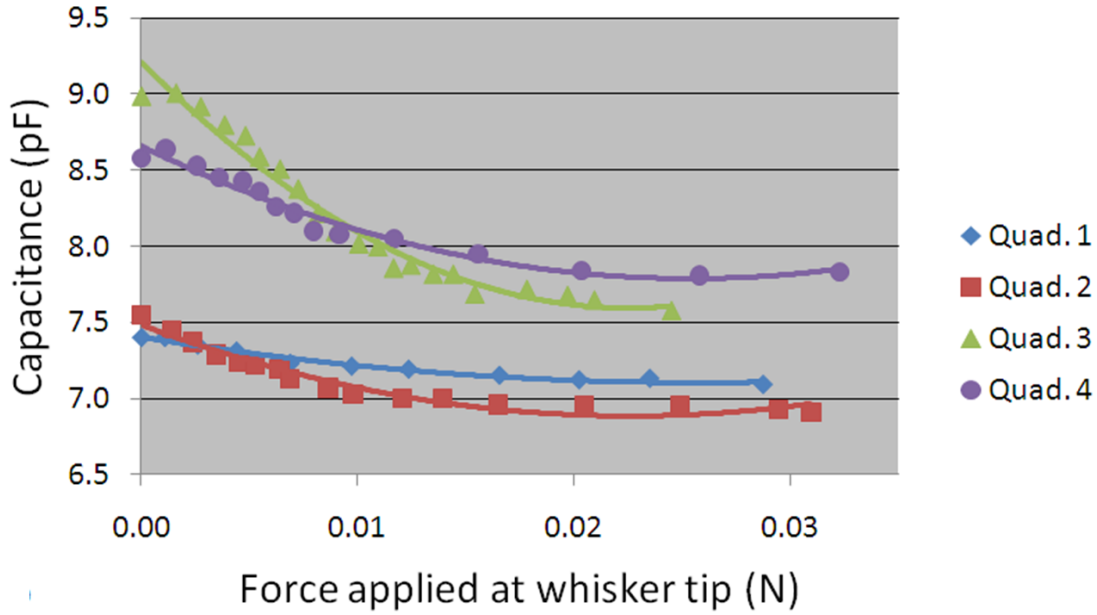

Figure 6.6: Force-capacitance measurement of each quadrant in the prototype sensor in separation [10]. 


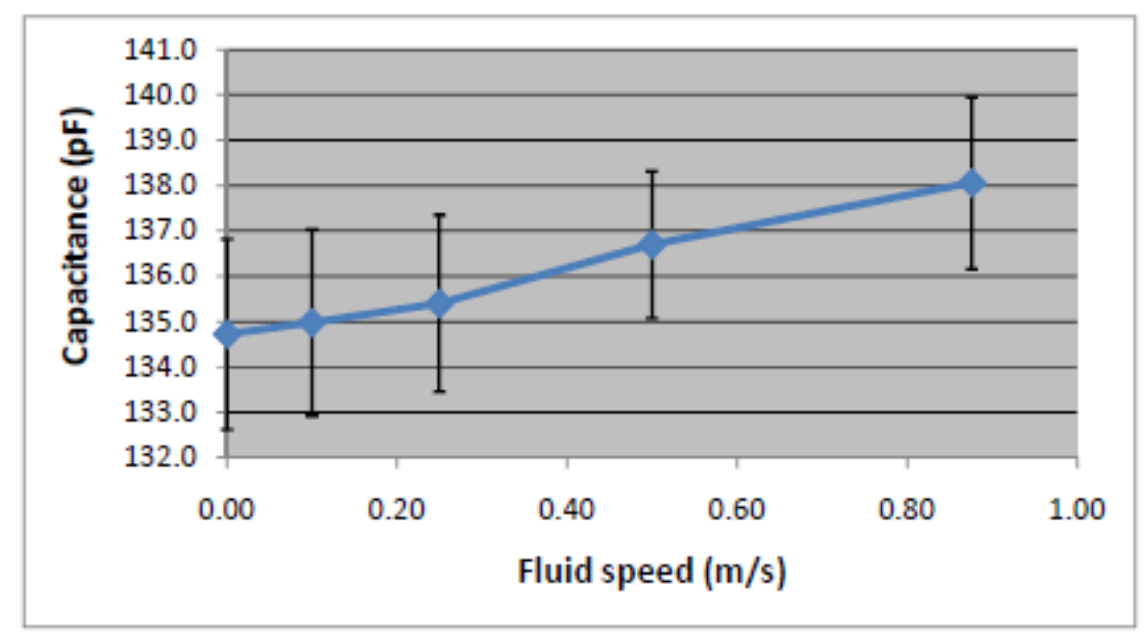

Figure 6.7: Prototype sensor output (quadrant 3) in water flow [11].

increase. The sensor exhibited hysteresis, failing to return to the same starting capacitance after each run and as a result measured capacitances increased during each test.

\subsection{Whisker-like Sensor Development}

Overall the testing conducted by Stocking proved the sensor design to be capable of measuring post deflections with repeatably. However the measured capacitances were small and the sensor had trouble in water testing. Further discussion of the results and methods for improvement are covered in the next section. All of the following work is the effort of the author.

\subsubsection{Discussion of the Prototype}

The prototype sensor suffers from several important problems. First, the output capacitance range was limited to $9 \mathrm{pF}$. The small capacitance measurements were caused in part by the epoxy waterproofing layer generating additional parasitic capacitances in series with the desired sensor capacitance of the gap. The sensor design assumed the capacitance could be modeled as a simple parallel plate capacitor 
governed by Equation 6.1.

$$
C=\frac{k \epsilon A}{g}
$$

where $\mathrm{C}$ is the capacitance, $\mathrm{k}$ the dielectric constant of the fluid in the gap, $\mathrm{A}$ the surface area of the plate, $g$ the distance between the plates, and $\epsilon$ the permittivity of free space $\left(8.85 \times 10^{-12} \mathrm{~F} / \mathrm{m}\right)$. In the sensor design the fluid forces acting on the post cause the inner cone to pivot changing the gap between the plates and thus changing the capacitance of the sensor. The epoxy waterproofing layers covering each plate introduced new material in the gap with a different dielectric constant. This creates three capacitors in series with the total capacitance given by:

$$
\frac{1}{C_{\text {total }}}=\frac{1}{C_{\text {edge }}}+\frac{1}{C_{\text {gap }}}+\frac{1}{C_{\text {edge }}}
$$

where $C_{\text {total }}$ is the actual capacitance of the sensor and $C_{\text {edge }}$ and $C_{g a p}$ are the capacitances caused by the water proofing layers and fluid respectively. Therefore if the edge capacitances are much smaller than that of the gap they dominate the total capacitance of the system thereby limiting the total capacitance range.

The range was also limited by the measurement process. Difficulties arose measuring the capacitance alone as a means of monitoring whisker deflections. The signal was small, inconsistent, could not capture frequency information. It was also unable to measure capacitance with other fluids such as water in the sensor gap. The dielectric constant of silicone oil is 2.5 [106] while water has a dielectric constant of 80 . This fluid change alone would generate a $32 \mathrm{X}$ increase in the capacitance.

Another issue arose from the PDMS membrane. It was inconsistent and severely under cross-linked. Silicone oil limits the cross linking in PDMS and is likely a key component to its structural weakness in the prototype. The membrane was actually sticky to the touch and clearly not fully set. The changes in base capacitance of the flume tests suggests it was not fully sealed and water was leaking into the sensor 
generating the capacitance increase as the dielectric increased.

\subsubsection{Sensor Excitation and Measurement}

The whisker deflection was previously measured by capacitance. The lack of range, reliability in the results, inability to measure dynamic whisker deflections, and inability to directly measure capacitance in non-inert fluids led to the redesign of the whisker deflection measurement modality. To measure whisker deflection, the whisker cone is excited with a sine wave at a desired frequency and amplitude provided by an Agilent Technologies model 33250A function generator, and the output peakto-peak voltage, corresponding to the whisker's position, is measured across a load resistor by a Tektronixs $2014 \mathrm{C}$ oscilloscope. Changing the measurement modality helps improve the ability of the sensor to detect whisker deflection. The effect of this change for the prototype sensor is shown in Figure 6.8 with a $330 \Omega$ resistive load. The sensor output increases with increasing input frequency and the difference between compression and separation of the plates exceeds $200 \mathrm{mV}$ at the higher frequencies, which is easily measurable. The sensor design shows deflections are most sensitive in the compression region as expected by the inverse relationship between capacitance and gap.

The goal is to maximize the voltage difference detected, with respect to input voltage, between maximum compression and maximum openness to improve sensitivity to whisker position (referred to as gain range) as opposed to the sensor capacitance. A secondary goal is to lower power consumption by choosing the lowest appropriate frequency for the sine wave, which will be eventually be generated through batterypowered backend electronics when the sensor is used in the field. It was eventually discovered that higher frequencies that lead to high output voltages do not necessarily lead to maximum resolution. To verify the new measurement modality and to improve sensing resolution, we developed a model of the sensor's electrical behavior across frequency (during the third revision of the sensor development). 


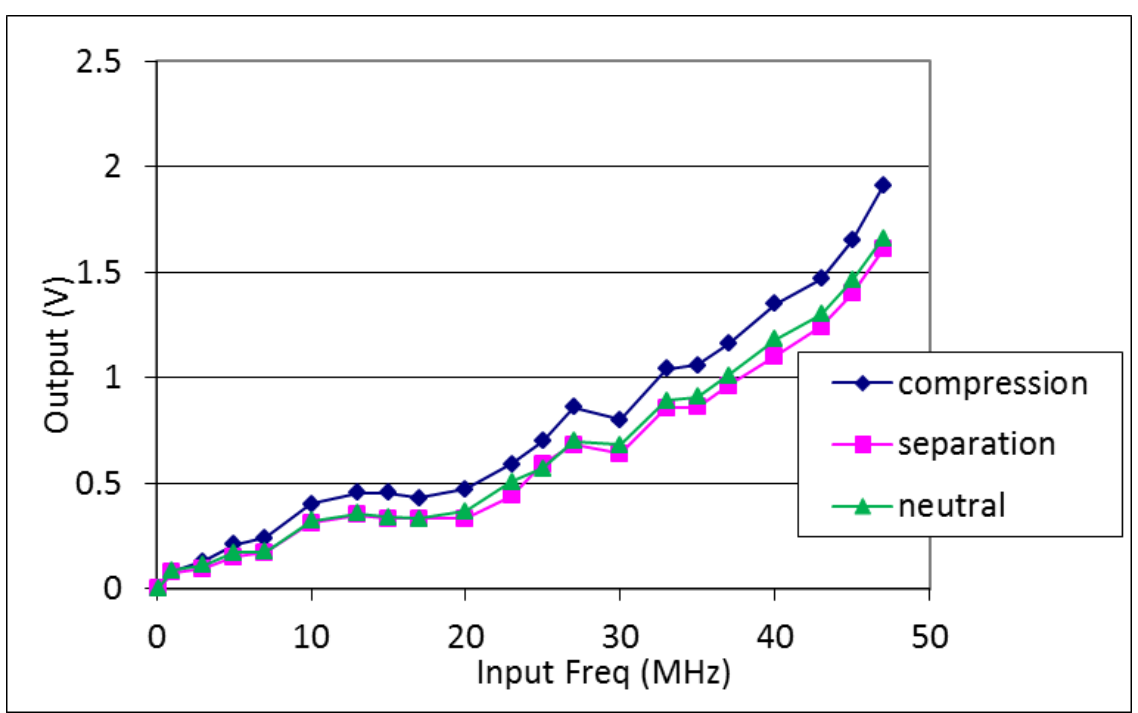

Figure 6.8: Revision 1 signal increases with frequency

\subsubsection{Revision 2}

After examining the results from the prototype, several design revisions were made. The small output signal was increased by enlarging the inner cone, replacing the silicone oil with water, and thinning the waterproofing epoxy layers. The PDMS membrane was created with more crosslinking (helped by the lack of silicone oil in the gap). Measurement of sensor output was changed from the capacitance meter to the sinusoidal input and measured across a load resistance. The improved sensor is pictured in Figure 6.9. This effort focused on improving the magnitude of the output signal.

The force tests up to $30 \mathrm{mN}$ showed the sensor never came close to full deformation, meaning the inner and outer cones were in no danger of touching. The increase in inner cone diameter would increase the surface area of the capacitor plate and reduce the gap size. Both these factors should increase the sensitivity of the whisker via Equation 6.1. Unfortunately the new inner cone molds failed to correctly generate the new diameter and only showed an increase of about $0.1 \mathrm{~mm}$. The transition from silicone oil to water had a drastic effect on the signal output. Attaching a $330 \Omega$ resistor to the sensor and exciting it with a $1 \mathrm{MHz}$ signal generated an output of over 


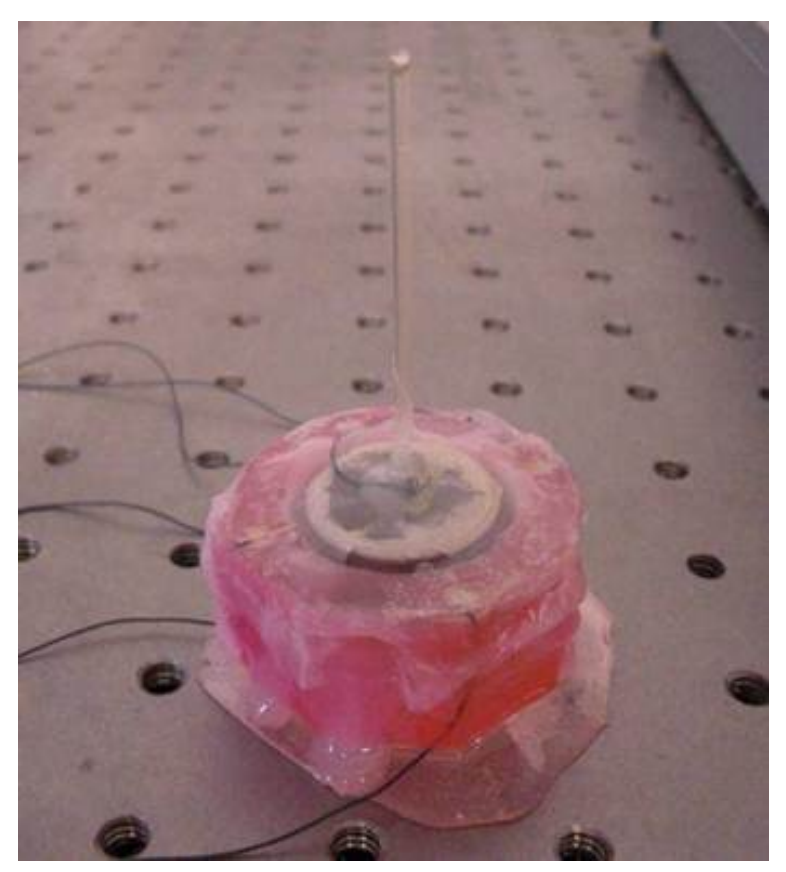

Figure 6.9: Revision 2 sensor

$400 \mathrm{mV}$ at rest compared to only $75 \mathrm{mV}$ when applied to the sensor prototype.

Examining the sensor response at a $10 \mathrm{MHz}$ input frequency with $6.58 \mathrm{~V}$ amplitude and $330 \Omega$ load provides the sensor range shown in Figure 6.10. Quadrants 1, 2, and 4 generate similar outputs as the post moves from full compression to separation. Quadrant 3 shows a smaller range with the same trend. This is caused by a thicker epoxy coating over this quadrant on the outer cone causing the parasitic capacitance to limit the quadrant's output. Using a thin sheet of aluminum foil and pressing it against the epoxy waterproofing layer, measurements from the capacitance meter showed the parasitic capacitance had a value around $10 \mathrm{pF}$, still quite small. The standard deviations shown by the error bars in the plot are small, under $5 \%$ the voltage output. However this is a significant increase over those shown in Figure 6.5. With the new measurement modality, the noise level in the sensor output also increased causing variations in the sensor output.

Over time the sensor output noticeably degraded as shown in Figure 6.11. Here the voltage change as the sensor is deformed in compression and separation from rest is plotted over time. Again the input frequency is $10 \mathrm{MHz}$ with $6.58 \mathrm{~V}$ amplitude 


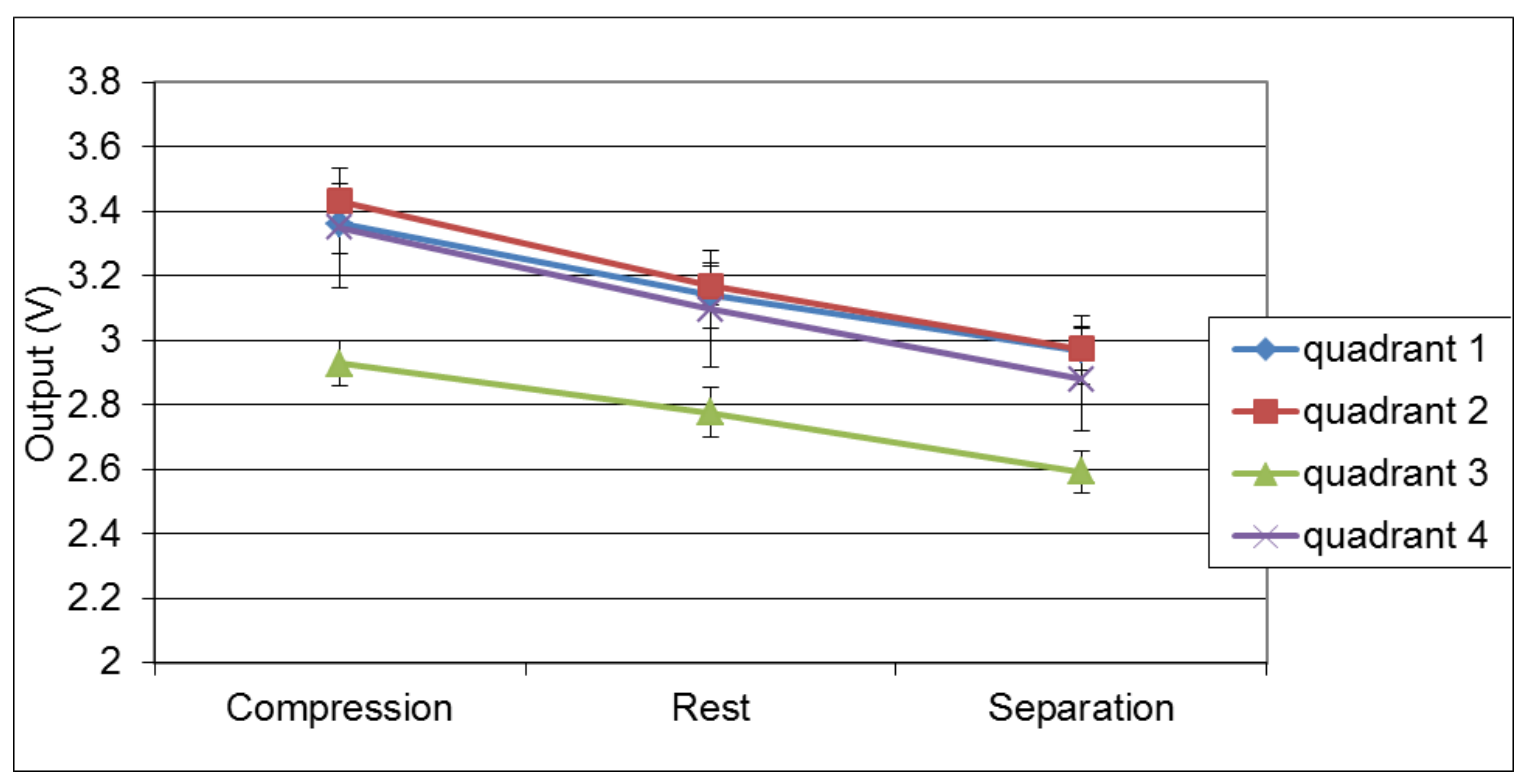

Figure 6.10: Revision 2 signal range for $330 \Omega$ load and input of $10 \mathrm{MHz}$ with $6.58 \mathrm{~V}$ amplitude.

and $330 \Omega$ load. Unfortunately different loading conditions examining the effects of frequency and resistive loads were tested between the first two data points on September 27 and November 22. It is impossible to determine exactly when the signal loss began and how quickly the sensor degraded. Due to the change in the quality of the output measurements, data taken between these dates is omitted as they likely improperly represent the sensor output.

Discovering the loss in signal quality, the PDMS membrane was removed revealing corrosion on the copper plates shown in Figure 6.12. The epoxy waterproofing layer failed to adequately seal the copper from the water in the gap. Epoxy coated steel has been shown to be prone to corrosion from defects in the epoxy coating unnoticeable to the eye [107] [108]. Passing a high frequency voltage through the copper plates likely aids in the corrosion.

While the second sensor revision showed drastic improvement in the output range and therefore sensitivity to fluid forces, several issues persisted. The noise level increased causing larger error in repeated measurements. This is attributed to the high input frequencies required to generate a large voltage output. The high frequency 


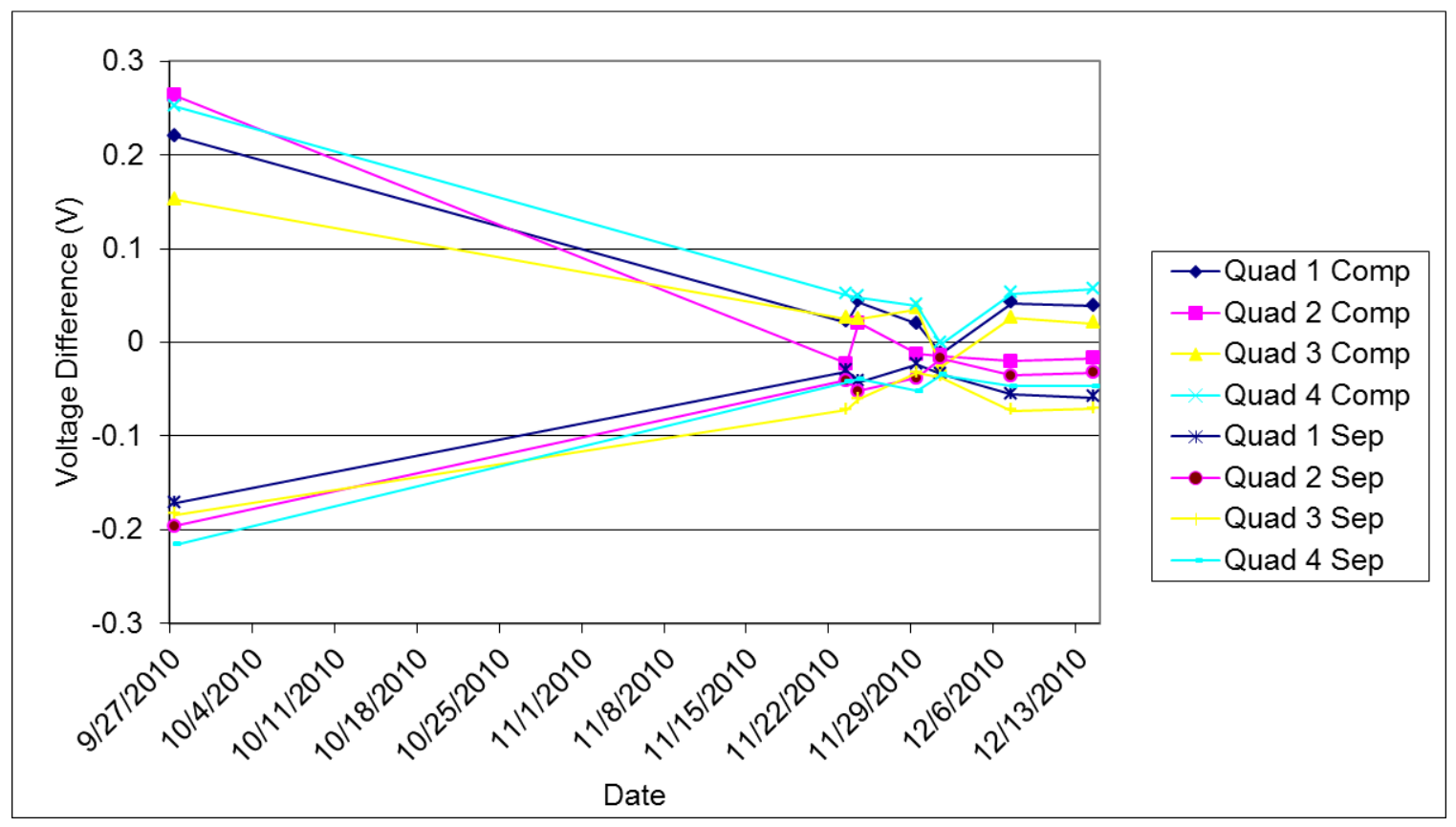

Figure 6.11: Revision 2 signal degradation

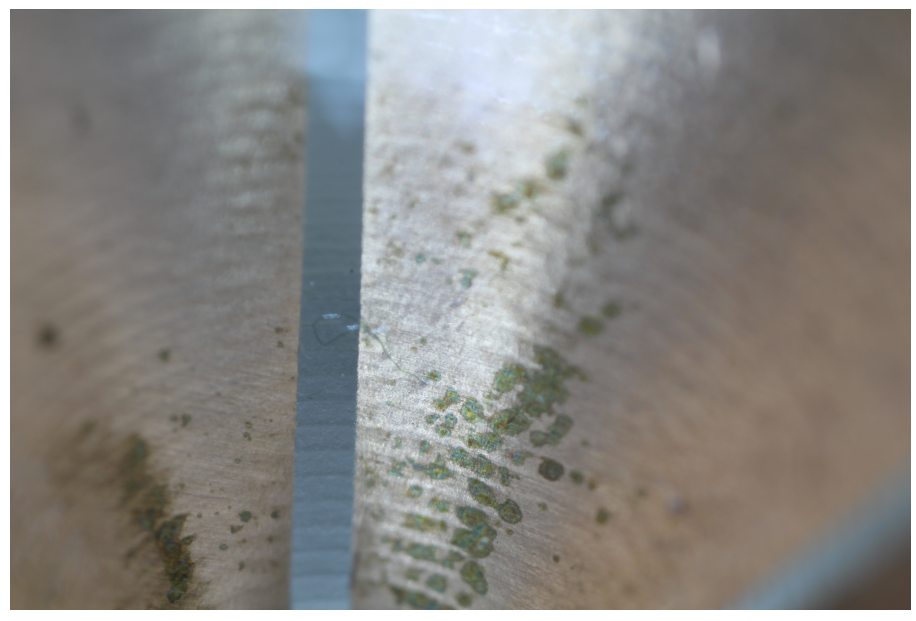

Figure 6.12: Corrosion on copper plates. 


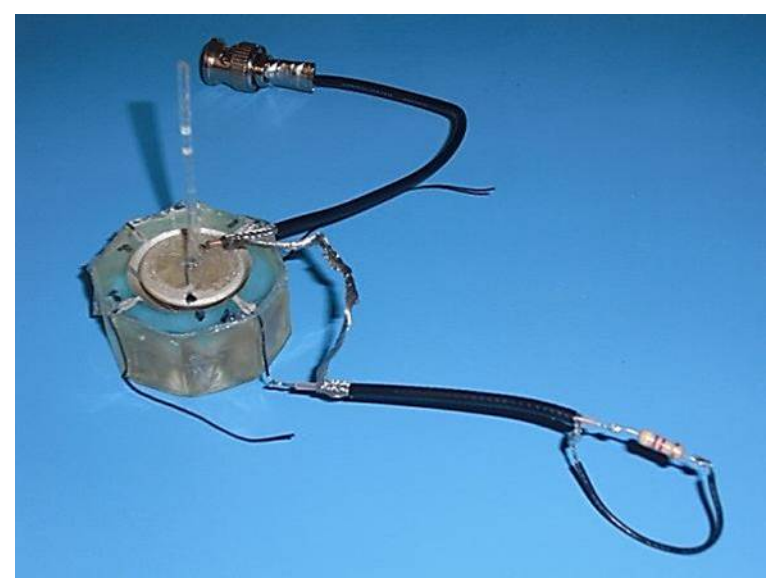

Figure 6.13: Whisker-like sensor revision three

generates electromagnetic interference picked up by the wires and then measured by the oscilloscope. The waterproofing epoxy layer was thinned thus increasing the parasitic capacitors (although it still remained small enough to limit the sensor output), however as a result it failed to protect the copper plates resulting in sensor failure. Although corrosion was apparent on the copper, no surface defects were visible on the silver epoxy plate of the inner cone.

\subsubsection{Revision 3}

Design improvements on revision three (Figure 6.13 shown with a coaxial cable connected to a resistive load) aimed to further reduce parasitic capacitances, improve the waterproofing layer, remove the corrosion issues, and remove the electromagnetic interference. This effort focused on maximizing the gain range. The goal is to maximize the voltage difference detected, with respect to input voltage, between maximum compression and maximum openness to improve sensitivity to whisker position (gain range) as opposed to the sensor capacitance/output as in revision two. By measuring the sensor deflection as gain range allows the results to scale for any input voltage and demonstrates the excitation frequency necessary to maximize the sensitivity.

Observing the lack of corrosion on the inner cone in revision two with the silver epoxy coating, the copper plates were removed from revision three and replaced with 
a coating matching that of the inner cone. The epoxy waterproofing was replaced with a commercial grade plastic wrap. This insured an even coating over all surfaces. Measuring the parasitic capacitance as previously described resulted in readings of $40 \mathrm{pF}$, a $4 \mathrm{X}$ improvement over previous coatings owing to the smaller thickness.

The small unshielded wires acted as an antennae. The measured signal would be comprised of up to $60 \%$ electromagnetic interference from the input signal for high frequencies (over $1 \mathrm{MHz}$ ). Therefore, the wires were replaced with coaxial cable. The resulting improvement in gain range is shown in Figure 6.14 for the unshielded wires, style 330 coaxial cable $2 \mathrm{~m}$ in length, and style 4620 coaxial cable of length $5 \mathrm{~cm}$. The results are from a single test demonstrating how the interference limited the change in signal as the whisker-like sensor deflected. The coaxial cable reduced the noise observed in the signal output to about $5 \%$. Some interference remains from the unshielded wire between the silver epoxy and coaxial cable. Both the 330 and 4620 cable should have equal protection over the electromagnetic interference and imply the length of the cable may play a role in the measured sensor response. All further testing used short RG-174/U coaxial cable.

The measured voltage gain range of the prototype sensor and the improved revision three sensor across a frequency range of $10 \mathrm{kHz}$ to $20 \mathrm{MHz}$ is shown in Figure 6.15. The gap was filled with silicone oil for each test. The silver epoxy, thinner waterproofing layer, and shielded wires attributed to the significant increase in signal quality and resolution. The improved design shows a $300 \mathrm{X}$ improvement in the gain range as the whisker deflects.

The sensor improvements and measurement modality also allow the sensor to operate in different fluid environments. Input frequency and the dielectric constant of the liquid in the gap both affect the gain range. The whisker sensor in its current form exhibits properties of a high pass filter. While a higher frequency initially yields a larger gain, it quickly reaches a maximum value. Also, a fluid with a larger dielectric constant in the gap yields a larger capacitance and therefore a higher output gain. 


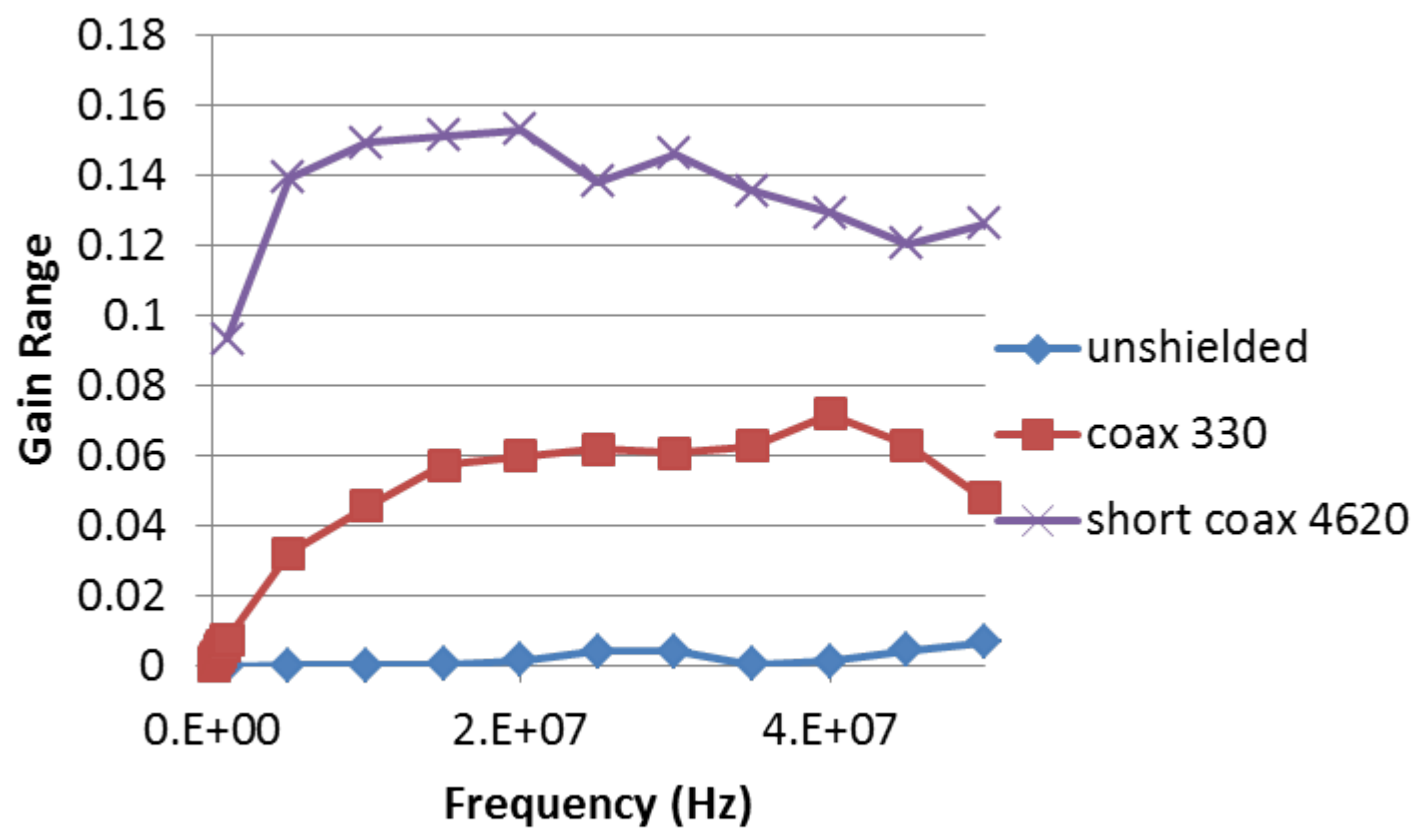

Figure 6.14: Wire effects on the sensor gain range.

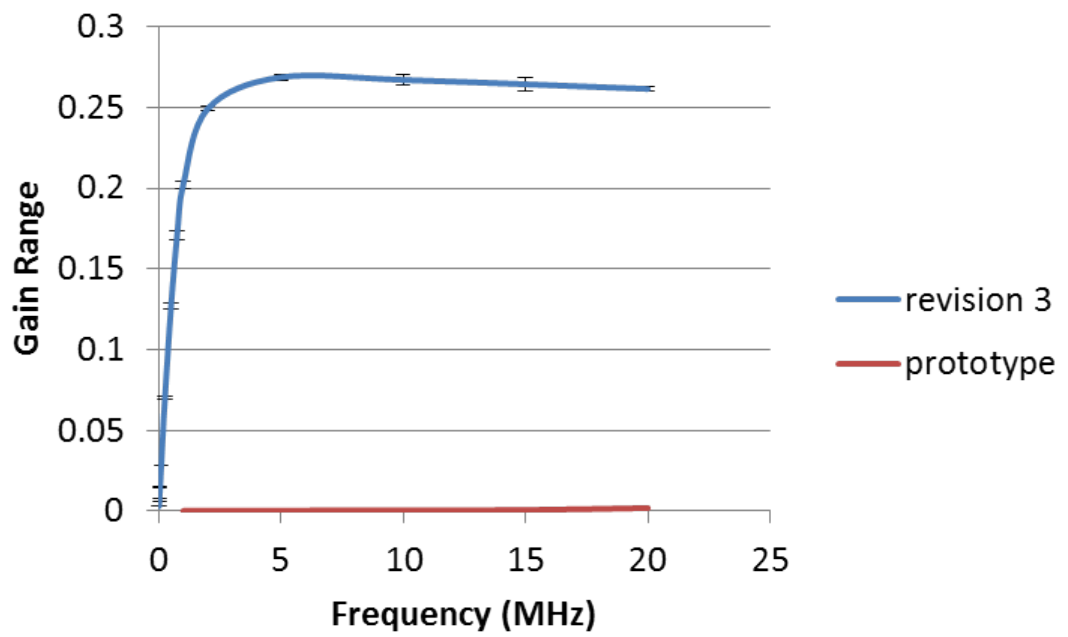

Figure 6.15: Improvement in gain range of revision compared to the prototype. 


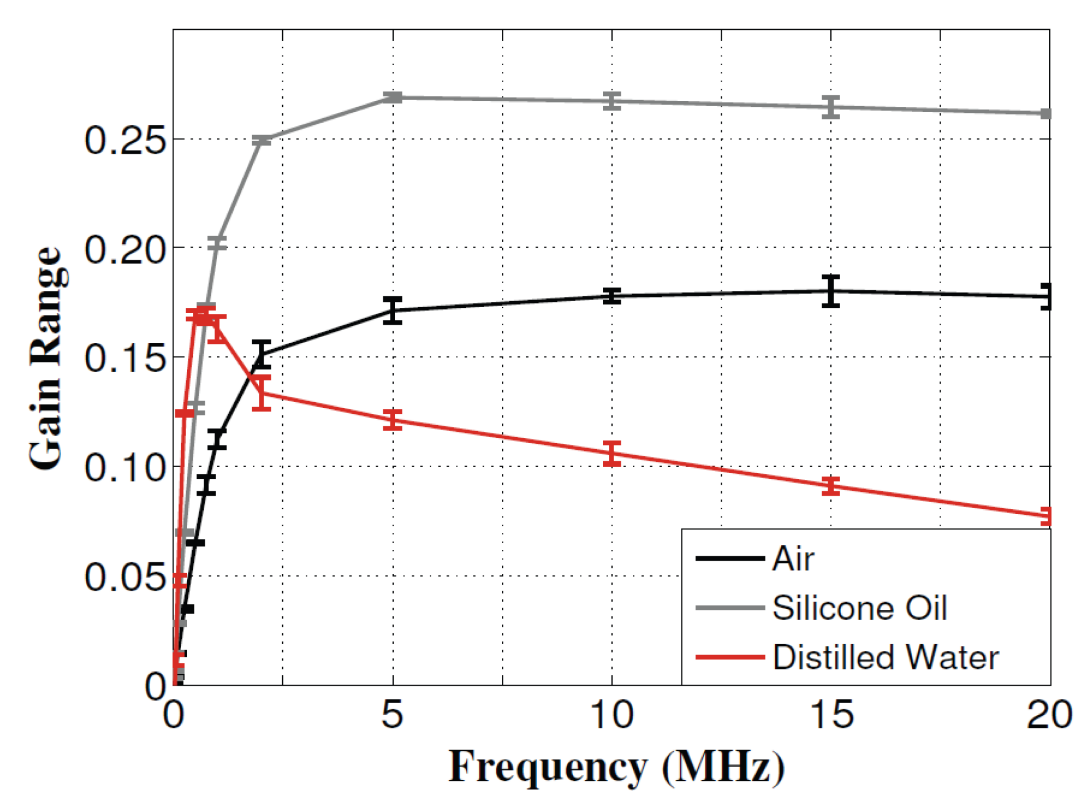

Figure 6.16: Gain range as a function of input signal frequency and fluid in the gap.

However, the gain range of the signal actually has a peak at a much lower dielectric material. The gain range of the improved sensor is illustrated in Figure 6.16 for air (dielectric 1), silicone oil (dielectric 2.5), and distilled water (dielectric 80) in the gap. Although distilled water has the highest dielectric (generating the largest gains) the gain range as the sensor deflects is lower than the other two fluids with much lower dielectric constants. By examining the gain range it becomes apparent that maximizing the capacitance of the sensor will not provide the best resolution.

Placing the revision 3 sensor in the water flume and attaching it to the same sting described in Chapter 4, measurements were made to monitor flow velocity and measure the frequency shedding of the wake of an upstream cylinder. The flow velocity ranged from 0 to $50 \mathrm{~cm} / \mathrm{s}$. The sensor response was measured on the quadrant facing the flow as in Stocking's test on the prototype. The output increases in magnitude as flow speed increases (Figure 6.17). The increase in voltage appears to be inversely proportional to the flow speed as expected from the capacitance equation. The noise levels were high contributing to the large error. This is attributed to the waterproofing layer examined later. 


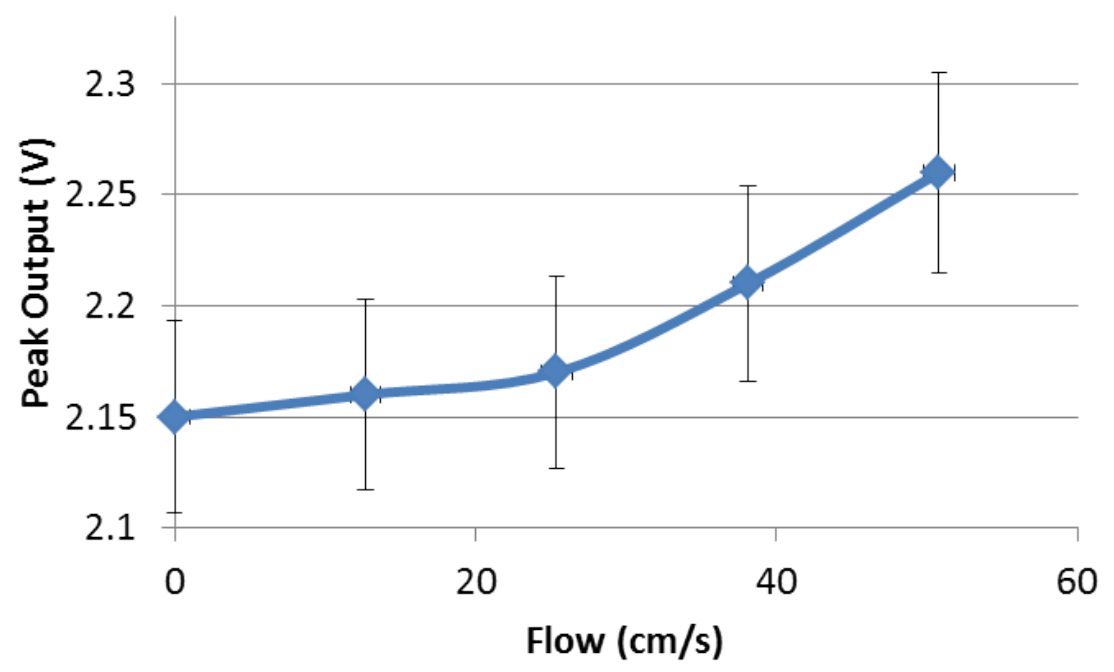

Figure 6.17: Peak voltage output of quadrant facing water flow direction as the flow velocity increases.

To test the whisker-like sensor's ability to capture the hydrodynamic signal of a wake, a $2 \mathrm{~cm}$ diameter cylinder was placed $5 \mathrm{~cm}$ directly in front of the sensor in the water flume running at $50.8 \mathrm{~cm} / \mathrm{s}$. The sensor response was monitored on a single quadrant in the cross stream direction, mimicking the measurements made on pinniped vibrissae in a cylinder wake. The post deflection was also measured at the midpoint by the laser vibrometer in the cross stream direction matching that of the tests conducted in Chapter 4. Unfiltered data of the peak quadrant output is shown in Figure 6.18.

The signal shows some noise again attributed to the waterproofing plastic. However an oscillatory pattern is also distinguishable. Using the equations from Chapter 4 the von Karman shedding frequency of the cylinder is estimated at $5.02 \mathrm{~Hz}$. Measurements from the vibrometer showed a peak at 5.0 Hz. Taking an FFT of the sensor data shows a peak at $4.88 \mathrm{~Hz}$ as shown in Figure 6.19. The discrepancy between the sensor frequency response and that of the vibrometer is likely a result of the short data collection time from the oscilloscope and noise present in the peak output. However the result is within $3 \%$ of the expected shedding frequency indicating the sensor is capable of monitoring the vortex shedding of the cylinder wake. 


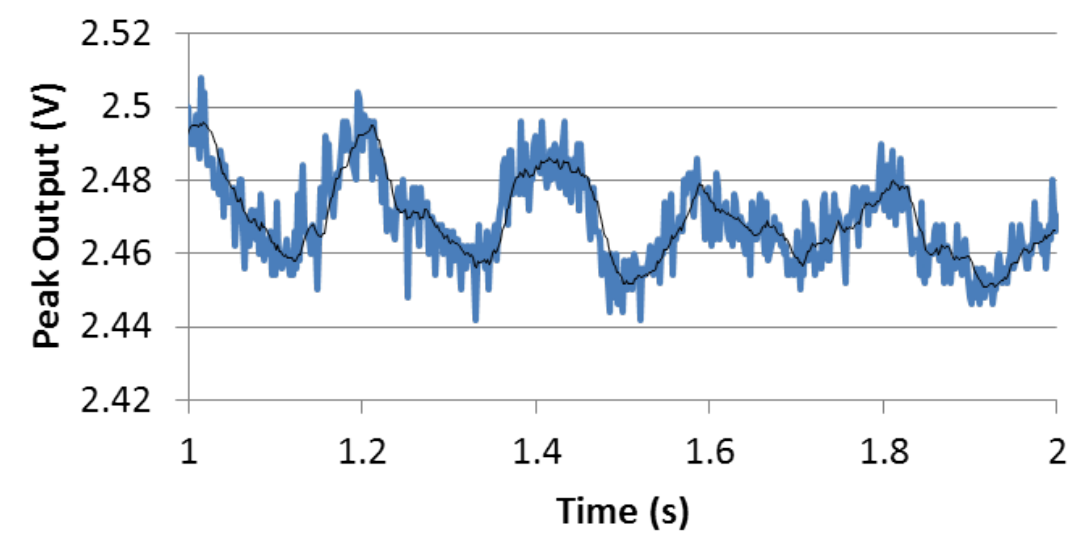

Figure 6.18: Peak values of sensor output measuring the cross stream deflections behind a $2 \mathrm{~cm}$ diameter cylinder.

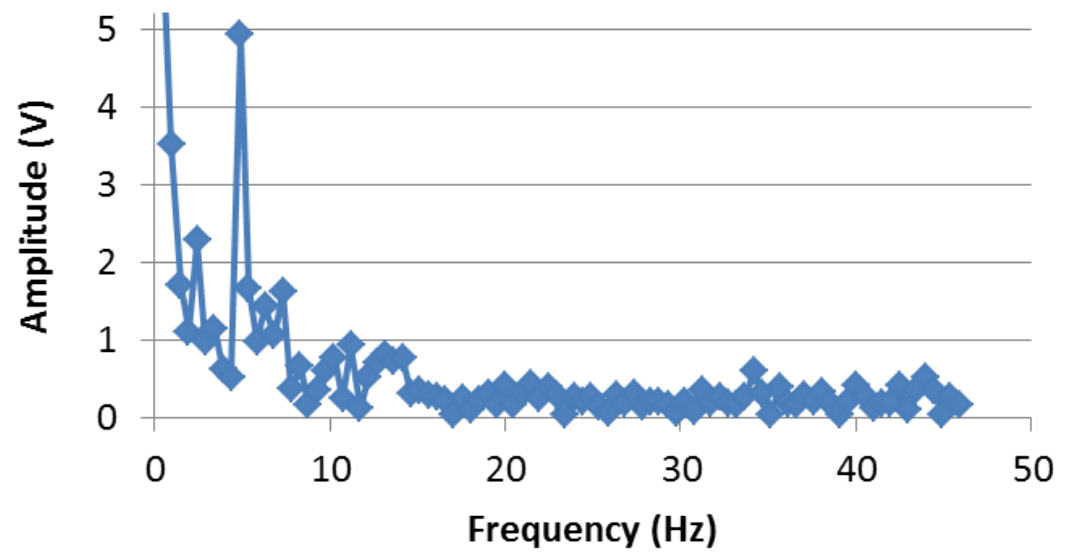

Figure 6.19: Cross stream quadrant frequency response to $5.02 \mathrm{~Hz}$ cylinder wake. 

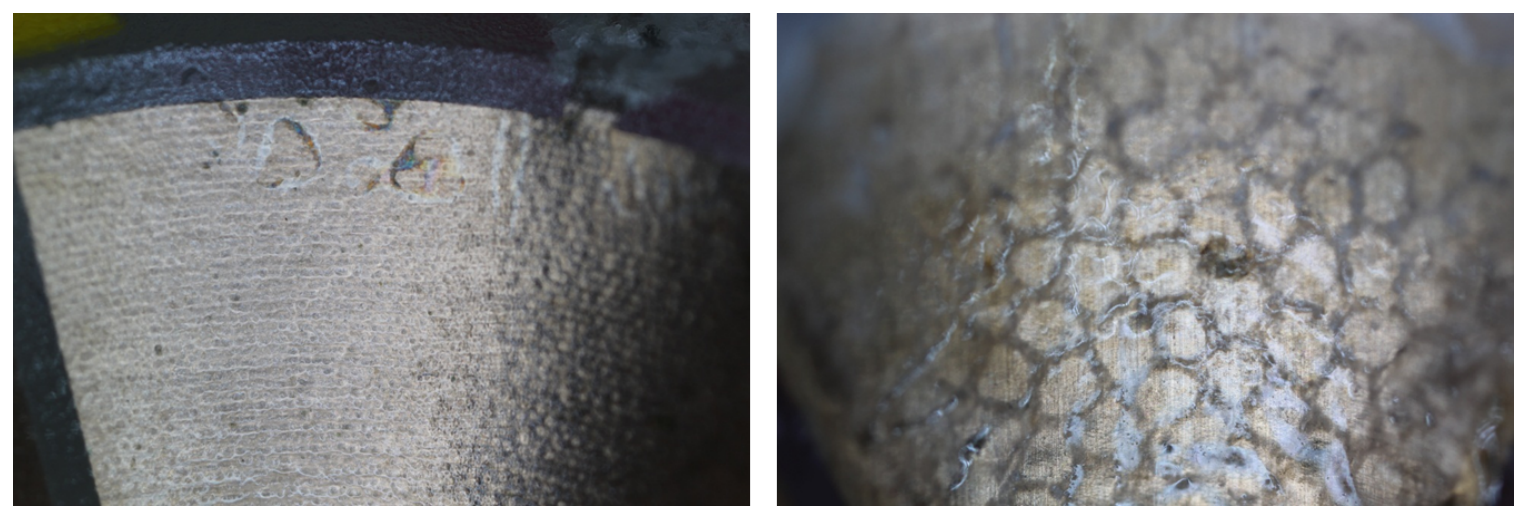

Figure 6.20: Degradation of waterproofing layer. Outer cone surface before testing (left) and after three months (right)

Despite the drastic improvement to the sensor response, the waterproofing layer continued to be an issue. The plastic covering again showed degradation over time as evident in Figure 6.20. The scaling pattern matches the small etching pattern in the plastic wrap. The loss in sensor response was not as pronounced as in revision two but the penetration of water into the plastic contributed to higher noise levels and lowered gain ranges.

\subsubsection{Sensor Theory}

The full circuit model over one quadrant with a resistive load and accounting for wire effects is show in Figure 6.21. The angle of deflection, $\alpha$, is measured as the angle between the inner and outer cone plates. To avoid shorting in the sensor, the metallic plates on the outer cone actually start at an offset distance, a, from the tip and extend to $\mathrm{b}$, the length of the side of the outer cone.

Previous circuit models of the sensor used parallel plate capacitor theory to predict the change in capacitance across the plates as the whisker deflected. The actual capacitor is an inclined plate capacitor conical in shape leading to Equation 6.3.

$$
C_{g a p}(\alpha)=\frac{2 \pi \epsilon k \sin (\alpha)(b-a)}{\alpha-0.5}
$$




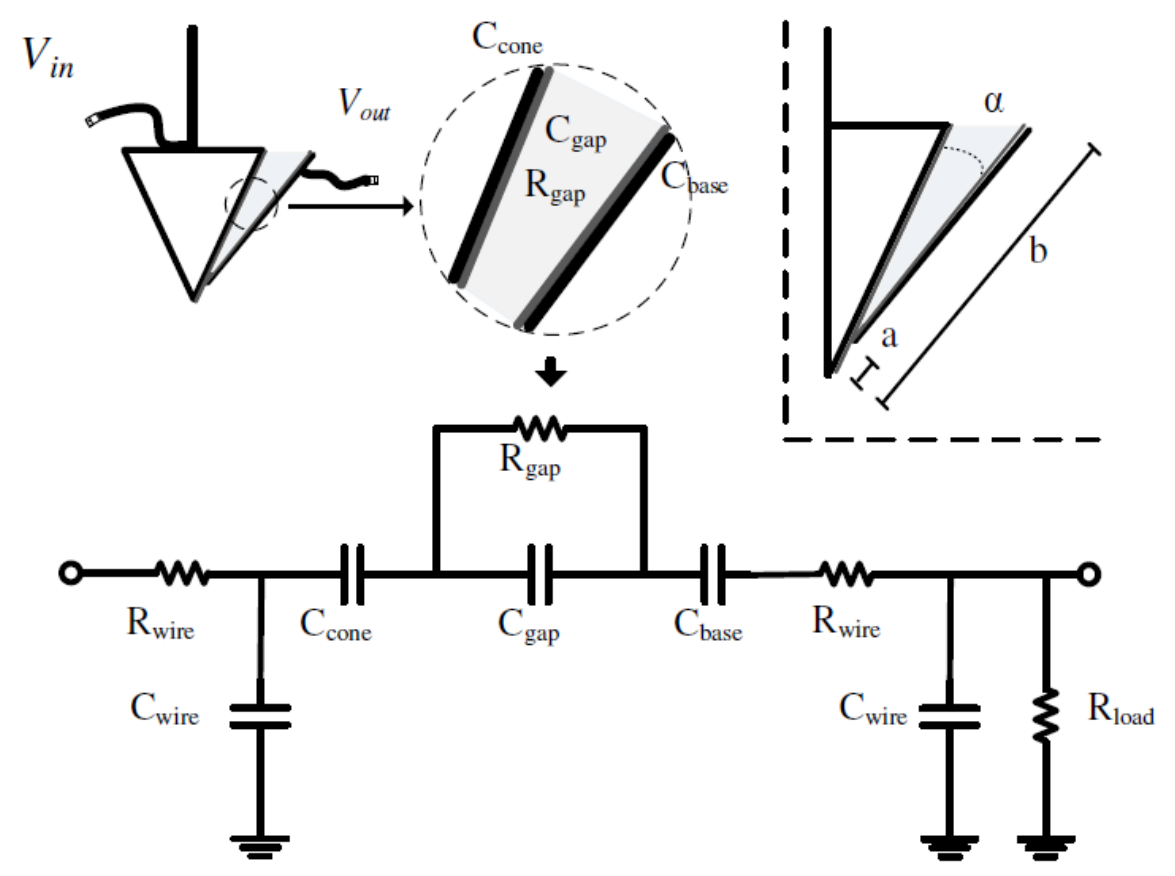

Figure 6.21: Schematic of the circuit model to estimate sensor performance.

In a similar manner the total resistance of the fluid in the gap, $R_{g a p}$, can be calculated from the resistivity:

$$
R_{\text {gap }}(\alpha)=\frac{r \sin (\alpha-0.5)(b-a)}{2 \pi \sin (\alpha)(b-a)}
$$

where $\mathrm{r}$ is the resistivity of the fluid in the sensor.

The complete circuit model is used to calculate the gain. The gain is the ratio of the output peak-peak voltage across a load resistor to the peak-peak input voltage applied to the sensor. The transfer function is as follows:

$$
\begin{aligned}
& H(s)=\frac{\left[\left(\frac{1}{s C_{\text {base }}}+\frac{1}{s C_{\text {cone }}}+\frac{R_{\text {gap }}}{s R_{\text {gap }} C_{\text {gap }}+1}+R_{\text {wire }}+\frac{R_{\text {load }}}{s C_{\text {wire }} R_{\text {load }}+1}\right)^{-1}+s C_{\text {wire }}\right]^{-1}}{R_{\text {wire }}+\left[\left(\frac{1}{s C_{\text {base }}}+\frac{1}{s C_{\text {cone }}}+\frac{R_{\text {gap }}}{s R_{\text {gap }} C_{\text {gap }}+1}+R_{\text {wire }}+\frac{R_{\text {load }}}{s C_{\text {wire }} R_{\text {load }}+1}\right)^{-1}+s C_{\text {wire }}\right]^{-1}} * \\
& R_{\text {load }} \\
& \frac{\overline{s C_{\text {wire }} R_{\text {load }}+1}}{\frac{1}{s C_{\text {base }}}+\frac{1}{s C_{\text {cone }}}+\frac{R_{\text {gap }}}{s R_{\text {gap }} C_{\text {gap }}+1}+R_{\text {wire }}+\frac{R_{\text {load }}}{s C_{\text {wire }} R_{\text {load }}+1}}
\end{aligned}
$$



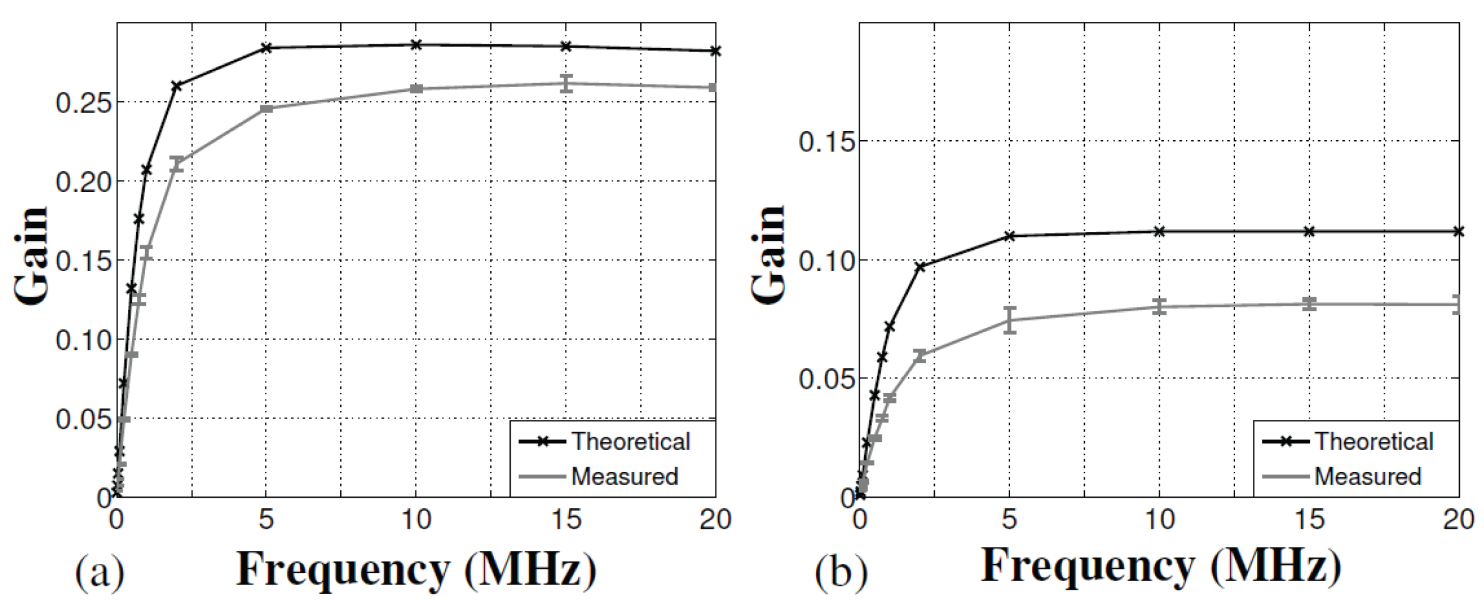

Figure 6.22: Comparison of gain between revision three and the theory for a) compression b)separation

where $s=j 2 \pi f, C_{\text {wire }}$ is the wire capacitance, $R_{\text {wire }}$ is the wire resistance, $C_{\text {cone }}$ is the parasitic cone capacitance from the seal, $R_{g a p}$ is the resistance of the medium, $C_{g a p}$ is the gap capacitance due to deflection, $C_{b a s e}$ the parasitic capacitance from the base, $R_{\text {load }}$ is the load resistance, and $\mathrm{f}$ is the frequency. $R_{\text {wire }}$ and $C_{\text {wire }}$ are added to the model due to the effects of the higher frequency sinusoid. By computing the gain of the sensor, it becomes possible to predict the range of the sensor output voltage for a given frequency and input signal as the whisker deflects.

The circuit model for revision three is in good agreement with the measurements. Results for the gain range with a load resistor of $4.62 \mathrm{k} \Omega$ and air in the gap are shown in Figure 6.22. The theoretical model is within $25 \%$ of the measured values and illustrates matching trends as a function of frequency. The overestimate of the theory may be attributed to some signal loss through the wire connections, electromagnetic interference, and mismatch in values for specific parameters such as the parasitic capacitances. Similar matching results and trends are observed with silicone oil and distilled water on the sensor gap

Examining the theoretical gain range as a function of dielectric constant for a 10 $\mathrm{MHz}$ frequency and $4.62 \mathrm{k} \Omega$ load, shows a peak at a low dielectric constant (Figure 6.23). This matches the results in Figure 6.16 where the silicone oils demonstrated 


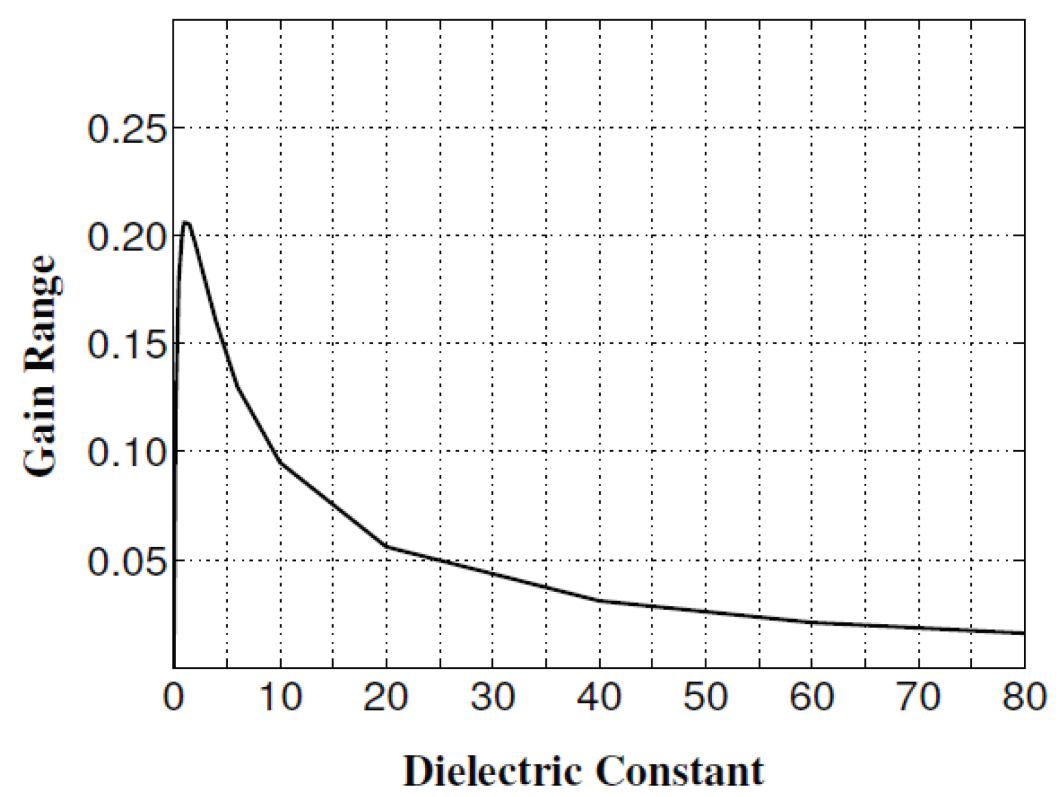

Figure 6.23: Theoretical gain range of the sensor as a function of dielectric material in the gap.

the largest gain range followed by air and then water with the smallest gain range at $10 \mathrm{MHz}$ despite the largest gain. By examining the gain range it becomes apparent that maximizing the capacitance of the sensor will not provide the best resolution.

The circuit model demonstrates reduced output signals in fluids of very low resistivity. In a fluid, such as salt water (resistivity of $0.2 \Omega \mathrm{m}$ ), the gain range is severely limited for all frequencies (gain range is nearly zero). The sensor is able to operate in a variety of fluid environments, however those with low resistivities require a PDMS membrane to separate the environment from a selected fluid within the sensor providing a better gain range to be encapsulated in the sensor gap.

The parasitic capacitances due to the ineffective waterproofing continue to limit the sensor response. Removing the waterproofing layer from the circuit model results in Equation 6.6. 


$$
\begin{aligned}
H(s) & =\frac{\left[\left(\frac{R_{\text {gap }}}{s R_{\text {gap }} C_{\text {gap }}+1}+R_{\text {wire }}+\frac{R_{\text {load }}}{s C_{\text {wire }} R_{\text {load }}+1}\right)^{-1}+s C_{\text {wire }}\right]^{-1}}{R_{\text {wire }}+\left[\left(\frac{R_{\text {gap }}}{s R_{\text {gap }} C_{\text {gap }}+1}+R_{\text {wire }}+\frac{R_{\text {load }}}{s C_{\text {wire }} R_{\text {load }}+1}\right)^{-1}+s C_{\text {wire }}\right]^{-1}} * \\
& \frac{\frac{R_{\text {load }}}{s C_{\text {wire }} R_{\text {load }}+1}}{\frac{R_{\text {gap }}}{s R_{\text {gap }} C_{\text {gap }}+1}+R_{\text {wire }}+\frac{R_{\text {load }}}{s C_{\text {wire }} R_{\text {load }}+1}}
\end{aligned}
$$

Using water as the fluid in the gap and varying both the input frequency and resistive load, a contour plot is generated to map the locations of highest gain range and therefore the largest sensitivity to deflections. The result showed impressive potential. The excitation signal frequency could be greatly reduced from the $\mathrm{MHz}$ range to Hz. This is aided by matching the excitation signal to an optimal resistance load to maximize the gain. The optimal combination for sensor signal quality and sensitivity occur with loads of 500-1000 ohms and frequencies below $700 \mathrm{kHz}$. The input frequency (and therefore output frequency as well) can be adjusted for the expected range of detection frequencies to reduce power consumption.

\subsubsection{Revision 4}

The failure of a waterproofing layer to properly protect the capacitance plates from corrosion over extended periods of time led to its removal. The corrosion issue was solved by gold plating the silver epoxy as shown in Figure 6.25. The waterproofing layer removal also boosted the sensor sensitivity by eliminating the parasitic capacitances limiting the signal as the whisker moved. Further improvements included adding SMA connectors for the coaxial cable allowing for a common ground and simplifying the wiring during testing. This reduced the electromagnetic noise generated when exciting the sensor in the $\mathrm{MHz}$ range.

These sensor improvements increased the gain range of the whisker by $50 \%$ at previously tested high frequency excitation signals (over $1 \mathrm{MHz}$ ). More importantly the changes to the whisker allowed for peak signal quality at much lower input fre- 


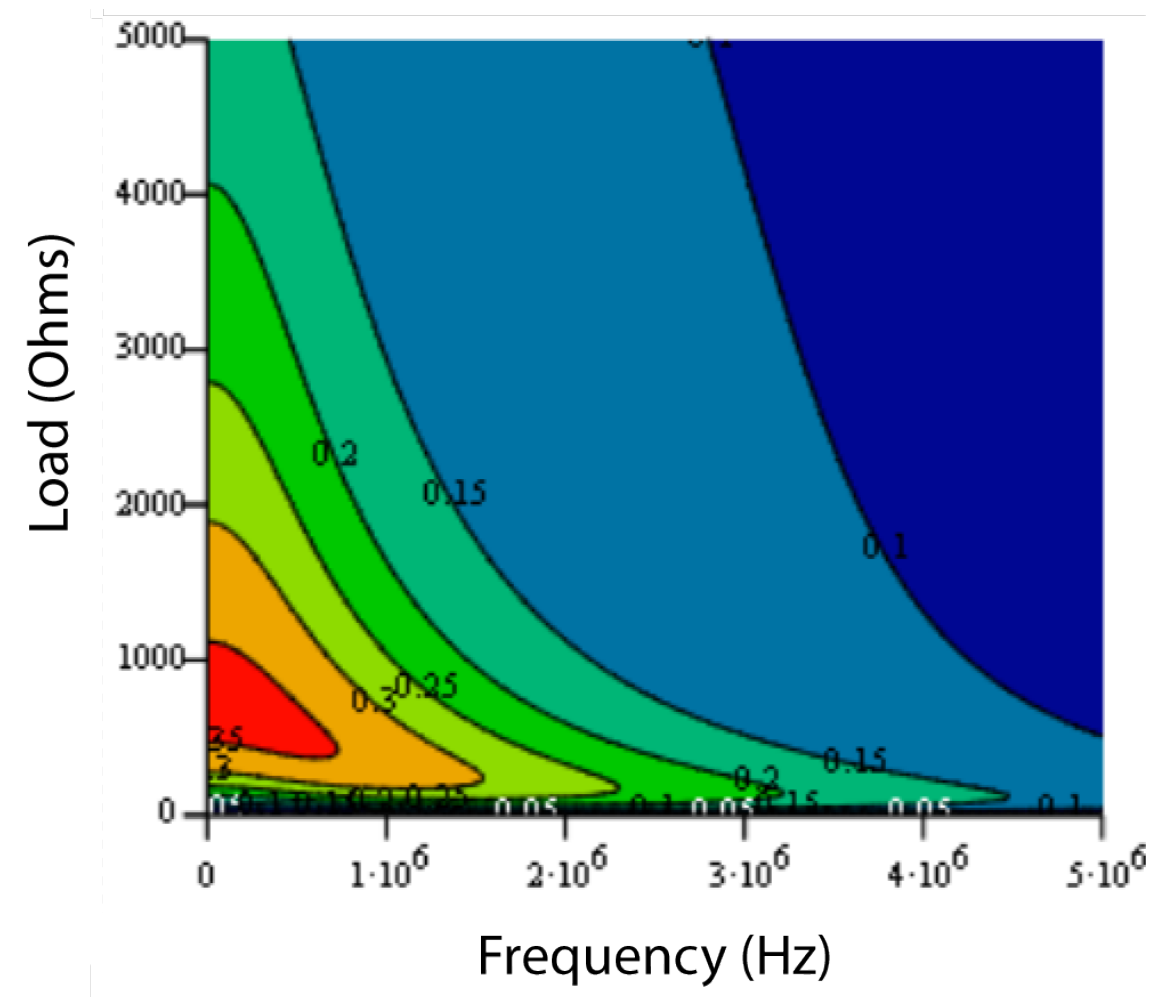

Figure 6.24: Contour plot of gain range for input frequency and load in distilled water.

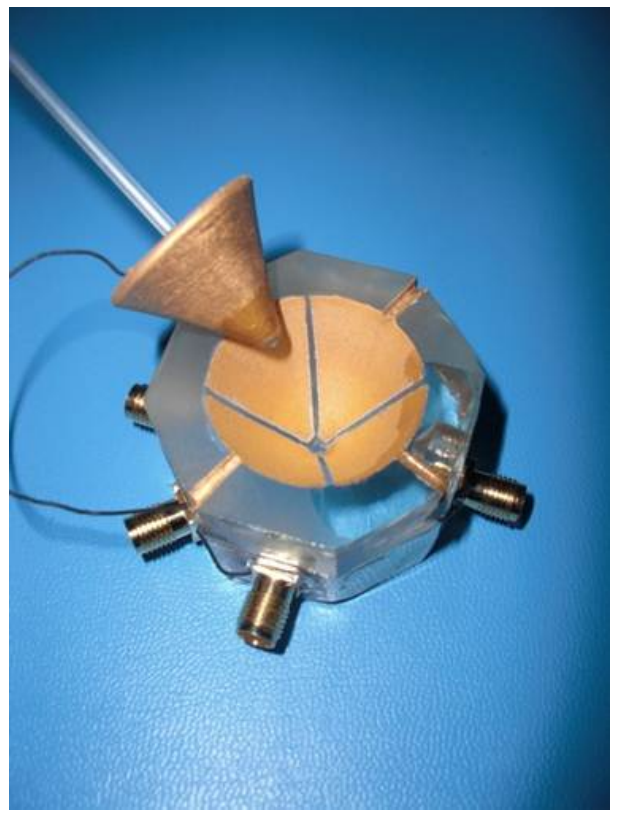

Figure 6.25: Revision four pictured without PDMS membrane. 


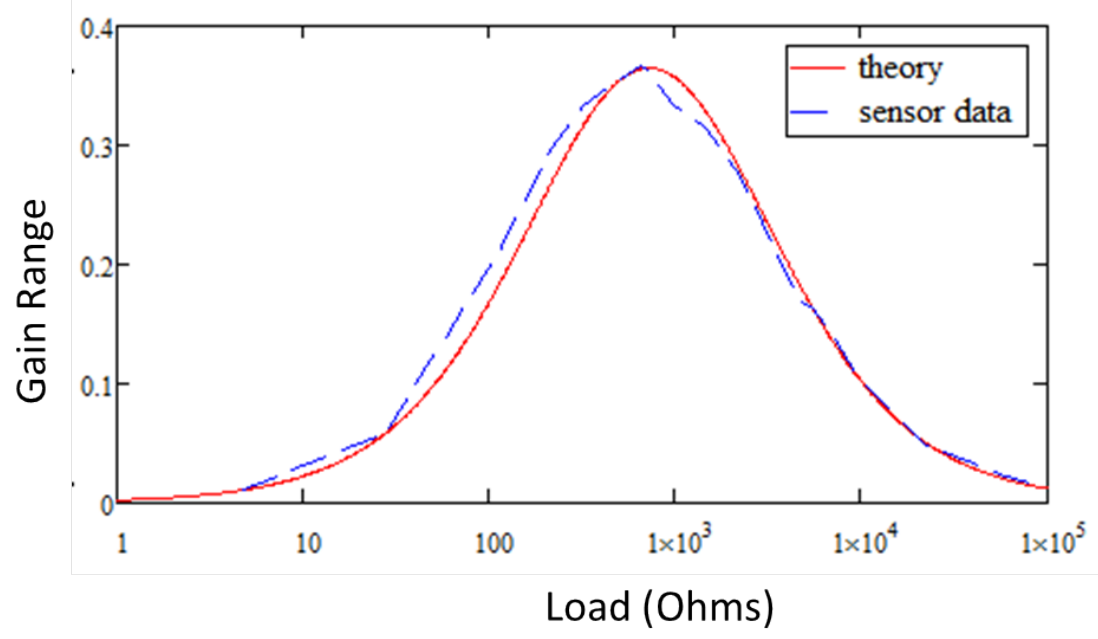

Figure 6.26: Experimental test of revision four compared to the model without parasitic capacitances with a $100 \mathrm{~Hz}$ input frequency showing gain range as a function of load.

quencies in the range of 100's of Hz. This provides large power savings and lower sampling rates to capture the whisker motion.

To verify the theoretical model the revision four sensor was excited at $100 \mathrm{~Hz}$ and the gain range was measured for loads from $2 \Omega$ to $100 \mathrm{k} \Omega$ in deionized water. Results are plotted in Figure 6.26. The variation in the sensor output was under $0.5 \%$ throughout the load range. The revision four sensor showed high repeatability and is nearly a perfect match to the theoretical model. Extended testing in water over several months showed no loss in signal quality or range implying the gold plating effectively removed the previous corrosion issues degradating the sensor output.

\subsection{Wake Measurement and Detection}

Having shown longevity and repeatability, revision four was placed in the water flume downstream of a cylinder in a similar fashion to the vibrissae tests. Cylinders of diameters 2,6 , and $8.9 \mathrm{~cm}$ were placed 19.7 and $51.2 \mathrm{~cm}$ directly upstream of the revision four whisker-like sensor mounted to the sting as shown in Figure 6.27. The sensor is placed up to 25 body lengths back of the wake generating object. Flow 

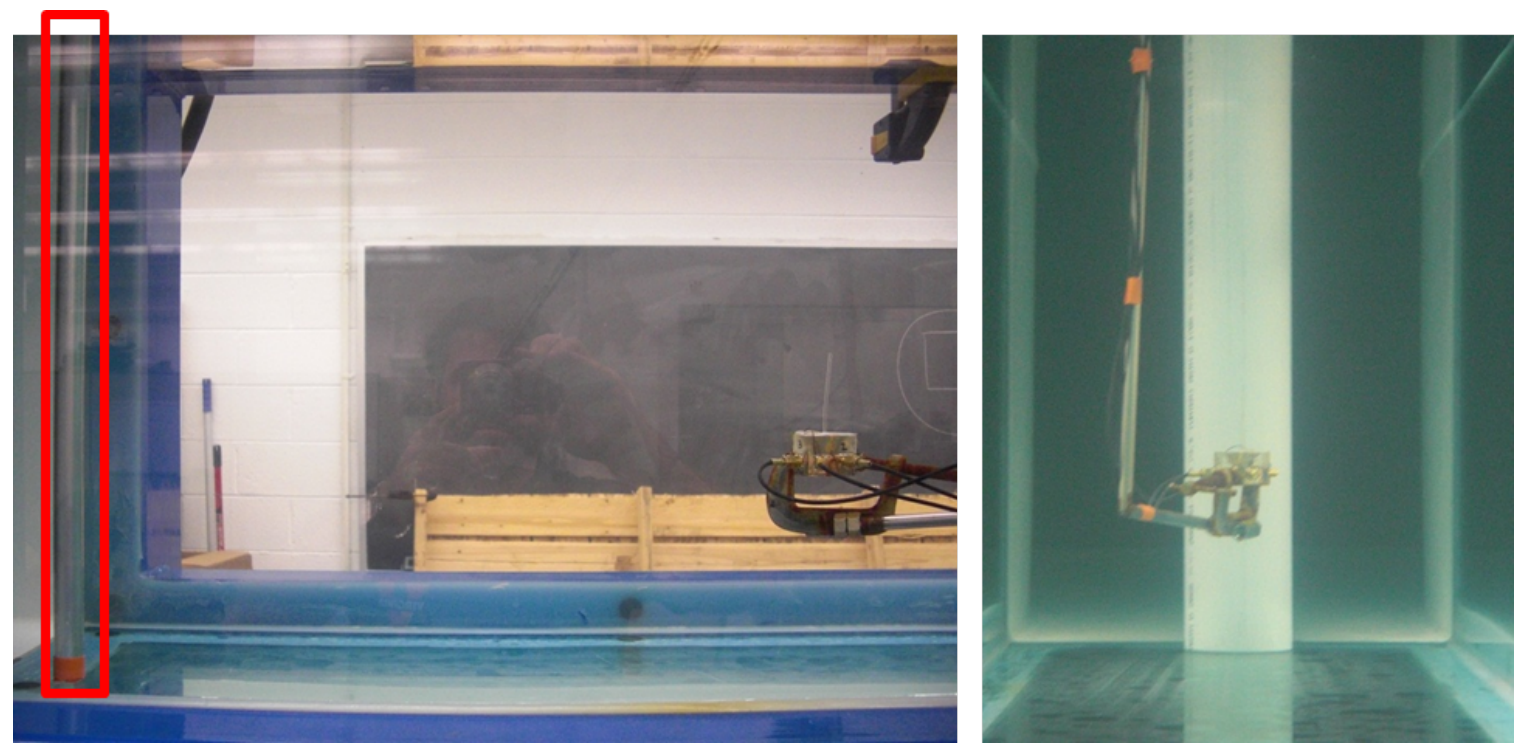

Figure 6.27: Revision four in water flume behind stationary cylinder. Right: Side view of sensor 25 body lengths behind $2 \mathrm{~cm}$ cylinder (in red box). Left: View looking upstream of sensor behind $8.9 \mathrm{~cm}$ cylinder.

speeds ranged from $15.2 \mathrm{~cm} / \mathrm{s}$ to $61 \mathrm{~cm} / \mathrm{s}$. The successful wake measurement by revision three was placed only $5 \mathrm{~cm}$ behind the cylinder or 2.5 body lengths.

Estimating the von Karman vortex shedding frequency assuming a Strouhal number of 0.21 , the cylinder wakes tested here should demonstrate frequency characteristics ranging from 0.34 to $6.03 \mathrm{~Hz}$. The sensor response was measured on two orthogonal quadrants with the Tektronix 2014C oscilloscope. Only two quadrants are monitored as each opposite quadrant makes a matching measurement. An example of measurements taken by the sensor is shown in Figure 6.28. In this case a $8.9 \mathrm{~cm}$ cylinder was placed $51 \mathrm{~cm}$ directly upstream of the whisker-like sensor. The unfiltered data from a quadrant facing in the cross stream direction and one facing the flow are shown on the top. The cross stream response shows an oscillatory pattern indicating the sensor is vibrating in the cross stream direction. No such response is observed in the quadrant facing the flow. Examining the frequency response, the cross stream quadrant detects a distinct peak frequency at $1.46 \mathrm{~Hz}$. The predicted shedding frequency of the cylinder wake is $1.1 \mathrm{~Hz}$.

The sensor was able to detect the cylinder wake shedding for all test cases. The 

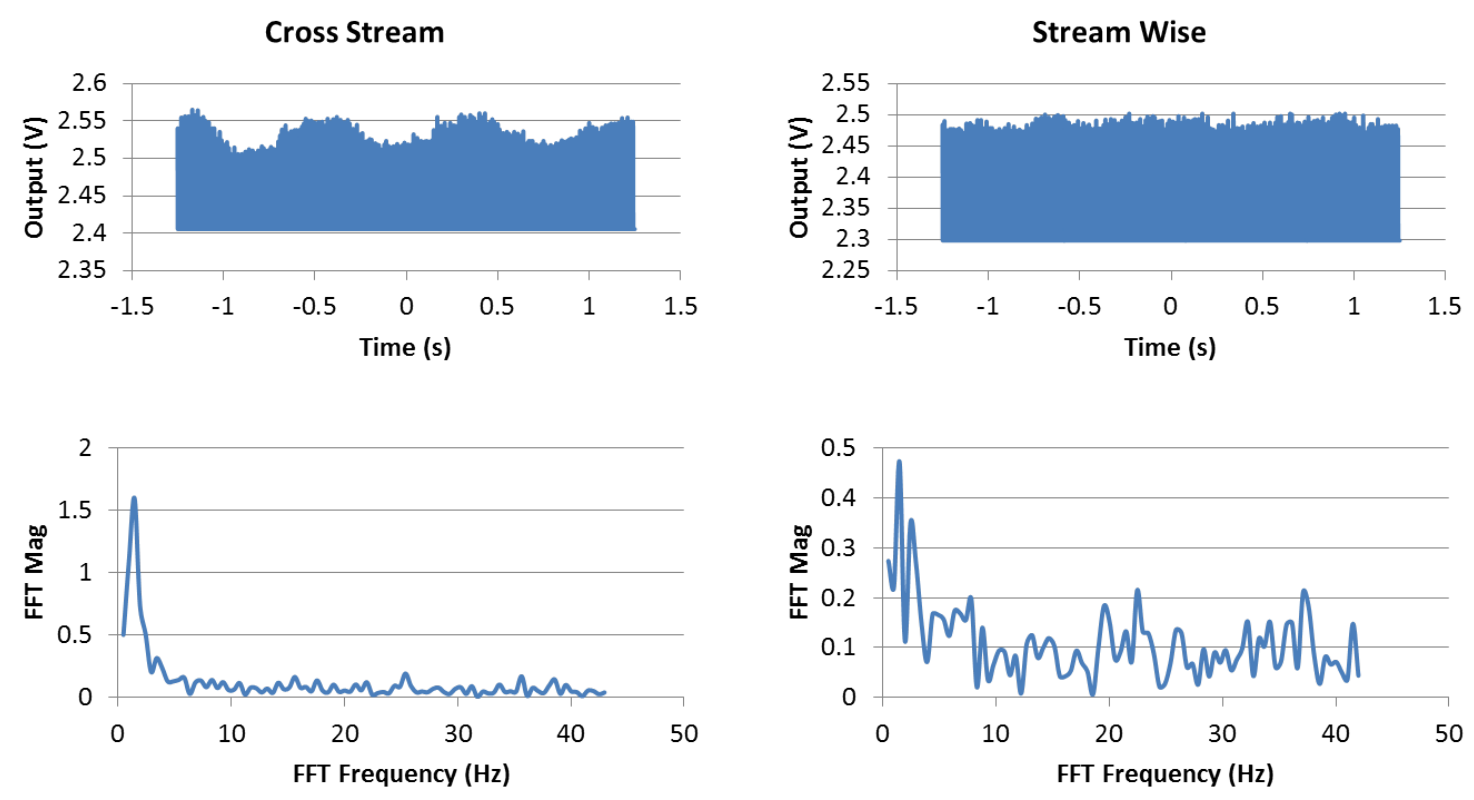

Figure 6.28: Sensor response to a $8.9 \mathrm{~cm}$ cylinder $51 \mathrm{~cm}$ upstream in a $46 \mathrm{~cm} / \mathrm{s}$ flow. Top: raw data from the cross stream quadrant (left) and quadrant facing the flow (right). Bottom: frequency response of measured data.

sensor's frequency response to the two extremes of frequency tested is shown in Figure 6.29. The left shows the response to a $2 \mathrm{~cm}$ cylinder in a $61 \mathrm{~cm} / \mathrm{s}$ flow. The Strouhal number predicts a wake shedding frequency of $6 \mathrm{~Hz}$ and the peak captured was 6.8 Hz. The right plots shows the response to a $8.9 \mathrm{~cm}$ cylinder in a $15 \mathrm{~cm} / \mathrm{s}$ flow. The sensor detected a frequency of $0.49 \mathrm{~Hz}$ compared to a predicted frequency of $0.34 \mathrm{~Hz}$.

In total the sensor was capable of detecting a peak frequency coinciding within 0.1$0.8 \mathrm{~Hz}$ that of the predicted frequency for all test cases. The measured response was always higher than that of the predicted values and much of the uncertainty is a result of the small data collection allowed by the oscilloscope. The wake characteristics were also always captured by the cross stream quadrant. This matches the results of the pinniped vibrissae wake monitoring from Chapter 4. A key distinction is the vibrissae are inclined to vibrate in the cross stream direction due to the flattened shape. Here the round post was able to capture similar information although at higher flow speeds.

Proving the sensor can capture the hydrodynamic properties of a wake, the next 

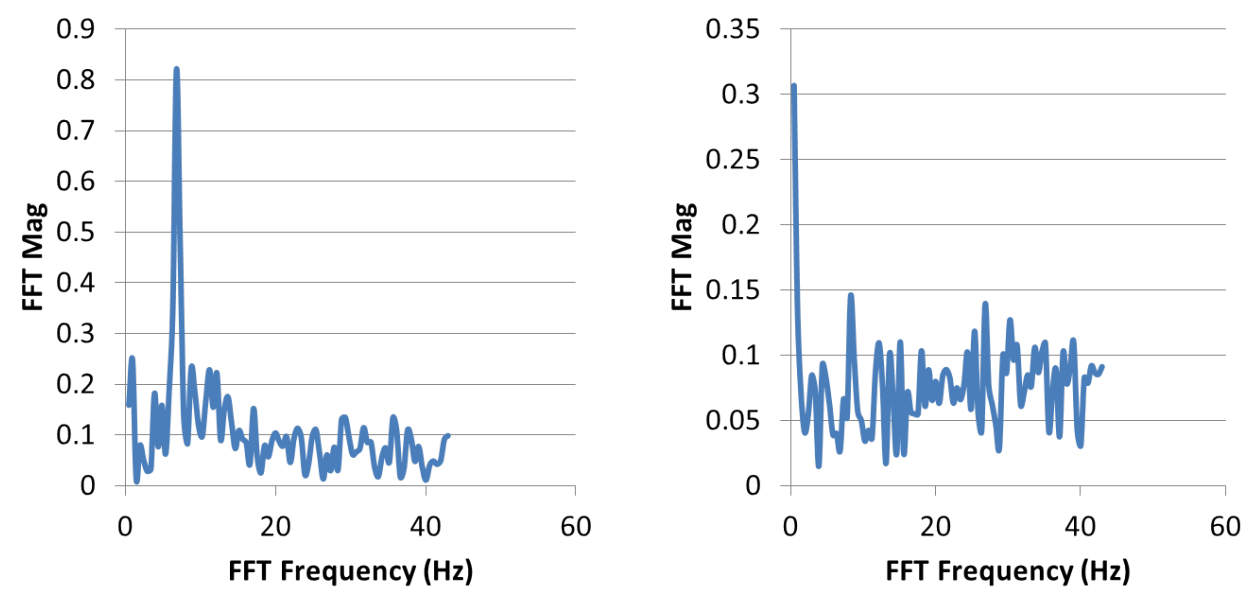

Figure 6.29: Frequency response of cross stream quadrant. Left: $2 \mathrm{~cm}$ cylinder in a $61 \mathrm{~cm} / \mathrm{s}$ flow. Right: $8.9 \mathrm{~cm}$ cylinder in a $15 \mathrm{~cm} / \mathrm{s}$ flow.

step is to determine the sensor's ability to detect a wake such as when chasing an object. Here the sensor remained in the middle of the water column attached to the sting as before. In these tests the cylinder was swept across the width of the tank. In this manner the sensor resides in the unobstructed free stream flow to start. The cylinder traverses the flow and the wake is carried downstream eventually striking the sensor. Measurements of the upstream quadrant and a cross stream quadrant peak voltage outputs are shown in Figures 6.30, 6.31, and 6.32 for a $2 \mathrm{~cm}, 6 \mathrm{~cm}$, and 8.9 cm cylinder traversing a $45.7 \mathrm{~cm} / \mathrm{s}$ flow $20 \mathrm{~cm}$ upstream of the sensor.

The stream measurement is taken from the front quadrant. Therefore an increase in voltage indicates a reduction in velocity flow observed by the sensor. The sensor experiences the free stream flow and then a reduction in the stream wise flow occurs as the cylinder obstructs the flow ahead of the sensor. There is a clear increase in the stream voltage for the 6 and $8.9 \mathrm{~cm}$ cylinders. A change in the cross voltage coincided with the stream increase. This likewise indicates the cylinder wake passing over the sensor. The wake was difficult to detect in the $2 \mathrm{~cm}$ cylinder case. No discernible voltage change occurred in the stream output but a short drop was consistently observable in the cross stream flow. The signal fluctuations increased with increasing cylinder size. The results here show the sensor is capable of detecting an 


\section{$2 \mathrm{~cm}$ Cylinder}

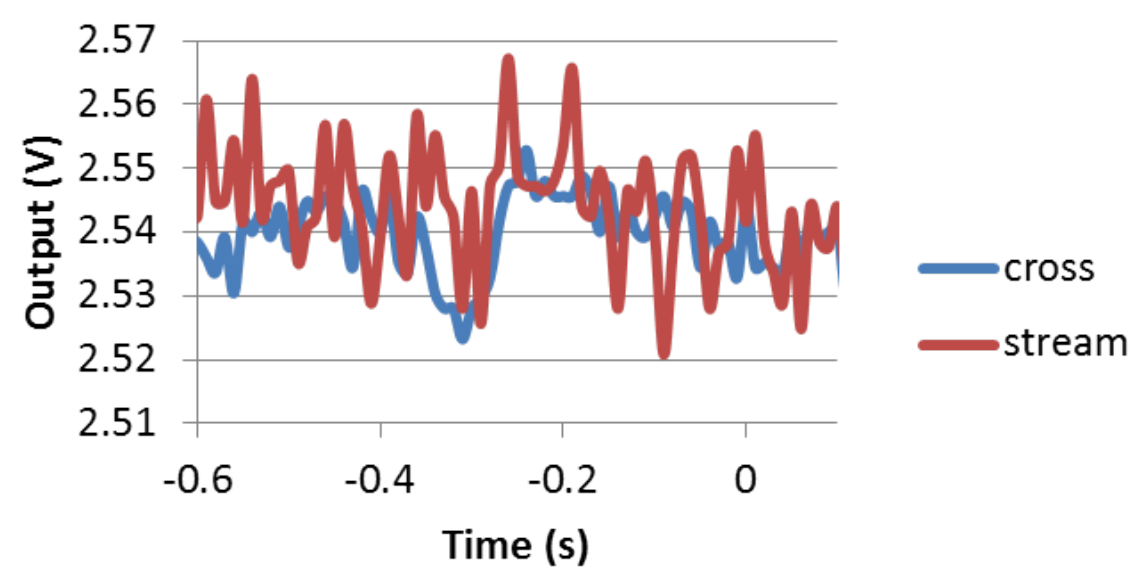

Figure 6.30: Sensor peak output as a $2 \mathrm{~cm}$ cylinder traverses the tank in a $45.7 \mathrm{~cm} / \mathrm{s}$ flow.

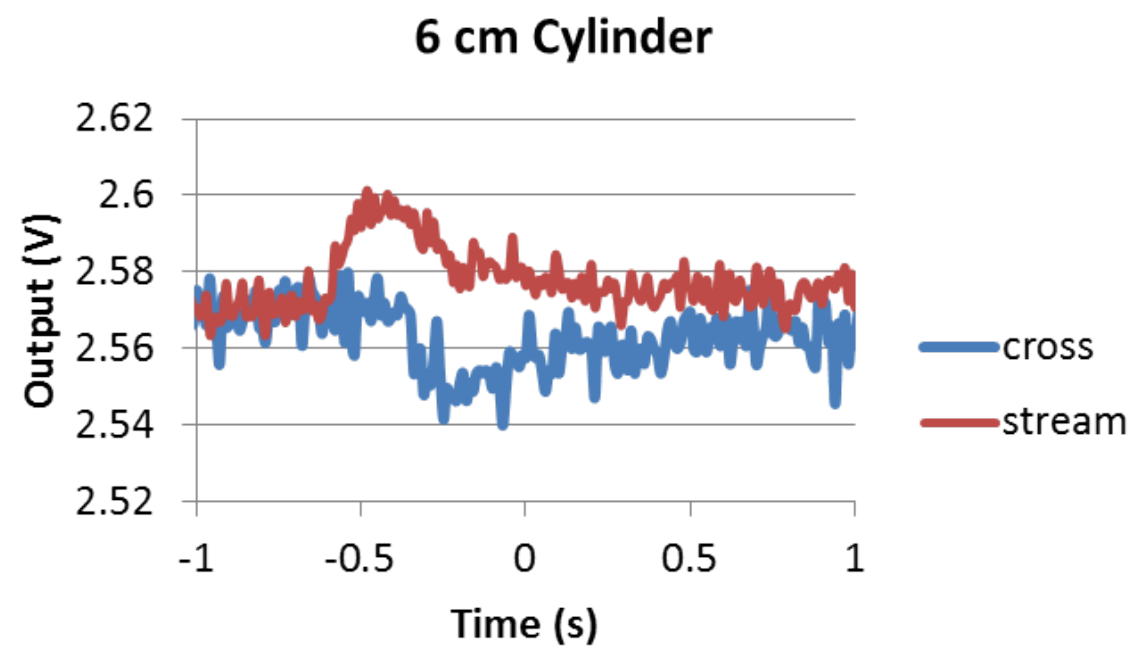

Figure 6.31: Sensor peak output as a $6 \mathrm{~cm}$ cylinder traverses the tank in a $45.7 \mathrm{~cm} / \mathrm{s}$ flow. 


\section{$8.9 \mathrm{~cm}$ Cylinder}

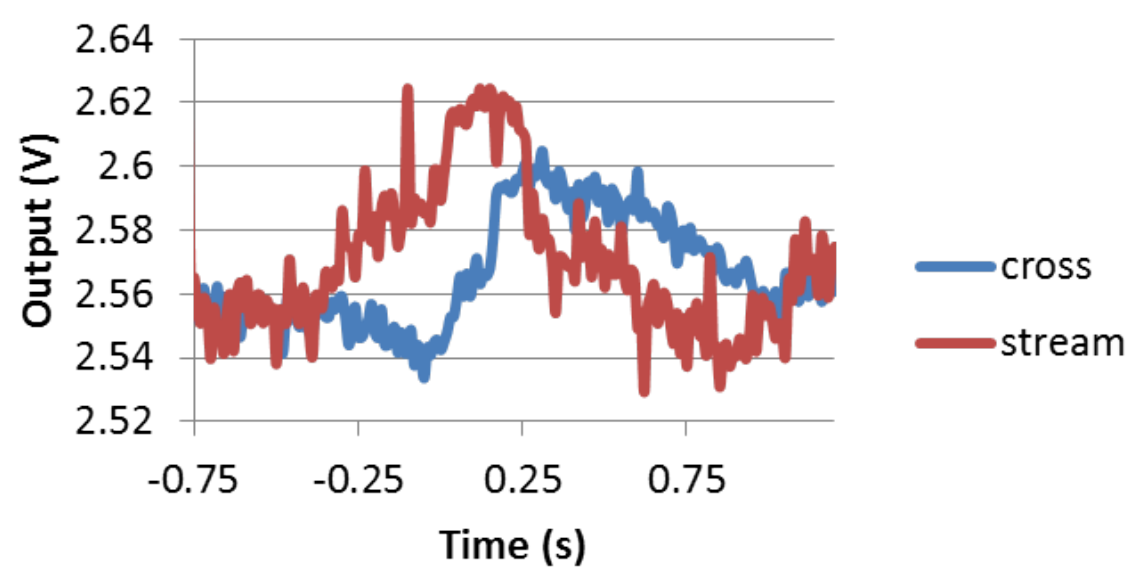

Figure 6.32: Sensor peak output as a $8.9 \mathrm{~cm}$ cylinder traverses the tank in a 45.7 $\mathrm{cm} / \mathrm{s}$ flow.

object crossing its path via the obstruction's wake.

\subsection{Discussion}

Examining several sensor ideas incorporating design features of trichobothria and vibrissae, a capacitance based cone-in-cone design was developed for the detection of hydrodynamic signals and future construction into a circular array. The sensor is capable of detecting fluid forces acting on the post or whisker in two orthogonal directions, each with added redundancy. The strongest signals occur across the quadrant under compression owing to the inverse relationship between capacitance and distance between the plates. Protecting the capacitance plates proved difficult and limited the sensor response through parasitic capacitances in series with the capacitance actually measuring post rotation. Simple capacitance measurements proved inadequate to detect the inner cone position leading to the measurement modality of exciting the inner cone with a sinusoidal input and measuring the output across a load resistor. The final sensor version removed the waterproofing protective layers all together and replaced the corroding plates with gold. 
Counter intuitively, maximizing the sensitivity was not accomplished by maximizing the capacitance such as incorporating high dielectric materials in the gap. Rather maximal gain range was best obtained at low frequencies (under $700 \mathrm{kHz}$ ) and with low dielectric materials. The circuit model correctly predicts the sensor output and can be used to optimize the design for many fluid environments. Limitations to this occur with materials of low resistivity such as salt water where the PDMS membrane must separate the low resistivity fluid from that within the sensor.

Revision four proved capable of detecting the shedding frequency of upstream cylinders as well as detecting a wake crossing the sensor. The sensor response to wakes behaved similar to that observed with the seal vibrissae although it was unable to monitor the wakes at speeds as low as the pinniped whiskers. The design easily captured frequencies under $0.5 \mathrm{~Hz}$ to $7 \mathrm{~Hz}$ and should be able to monitor higher ones as well.

\subsection{Summary}

The goal of this chapter was to develop a biologically inspired fluid motion sensor drawing from the capabilities and features found in nature, as discussed in Chapters 3 and 4. Reviewing the research questions presented at the start of the chapter, the cone-in-cone design is well suited to compactly be arranged into a circular array. Revision four showed no signs of deterioration after months of testing in the chlorinated tap water of the water flume. The four quadrants of capacitor plates allow for measurement in two orthogonal directions. Revision four showed only a $0.5 \%$ uncertainty in output when repeatedly loaded under the same conditions. The whisker-like sensor was able to detect cylinder wake frequencies and cylinders crossing the water column ahead of the sensor. Throughout testing the sensor experienced numerous mechanical disturbances and even shocks with no casualties. The only damage was the detachment of a wire or an SMA connector, both easily soldered back into place. 


\section{Chapter 7}

\section{Sensor Array Development and}

\section{Deployment}

The end result of this thesis effort is the placement of the whisker-like capacitance sensors on a harbor seal tracking a hydrodynamic signal. Efforts led by Dr. Colleen Reichmuth at the Long Marine Lab at the University of California Santa Cruz have led to a harbor seal, Sprouts (NMFS marine mammal permit 14535), trained to chase an underwater object. The animal is blindfolded and uses only its vibrissae to obtain information about the direction and speed of the chase object.

The objective is to develop and characterize both a suitable chase object and means of packaging the sensors in such a manner they do not interfere with the harbor seal's own sensing capabilities, but are still able to capture the same information. The chase object needs to be self-propelled, capable of working in salt water, and run at a high enough velocity to avoid being overtaken by the chasing animal. Characterization of the chase vehicle is conducted in a water tunnel using particle image velocimetry (PIV) to determine the type of hydrodynamic signal observed by the harbor seal.

A Wake Information Detection and Tracking System (WIDTS) is developed to carry an array of sensors on the seal. Effort focuses on determining a suitable size, shape, and placement of the WIDTS through the use of numerical modeling and 
field testing. The major contribution of this chapter is the design, construction, and testing of a sensor array platform for attachment to a harbor seal, which ties into the thesis statement by developing a system to monitor the hydrodynamic signal of a self propelled object. Research questions answered in this chapter include:

- How should the sensor array be packaged and attached to a swimming harbor seal?

- Will the WIDTS disrupt the harbor seal's tracking capability?

- Can the WIDTS detect/track wakes?

\subsection{WIDTS Development}

\subsubsection{Design Parameters}

The WIDTS is meant to be a single unit, packaging the sensors, electronics, power supply, and data collection into a self contained system for deployment in water. The WIDTS must be compact, hollow, waterproof, and array the sensors in a manner that can track the fluid motion around the entire body.

The cone-in-cone design of the sensor allows a group of sensors to be arranged radially around a body (Figure 7.1). In this manner, four sensors one on the top, bottom, left, and right are fitted around a ring. A second ring offset by 45 degrees about the axis of symmetry behind the first ring generates two arrays consisting of eight sensors surrounding the WIDTS body at 45 degree intervals. This spacing insures flow is monitored about the entire system and allows enough spacing to ensure the sensors do not interfere with each other. The design should allow the rings to be interchangeable providing simple replacement in the event of a sensor failure.

The WIDTS must be a self contained unit. To protect the electronics and power supply the system is hollow. Therefore the design needs to minimize the body size while maximizing the space inside for the printed circuit board (PCB) containing the 

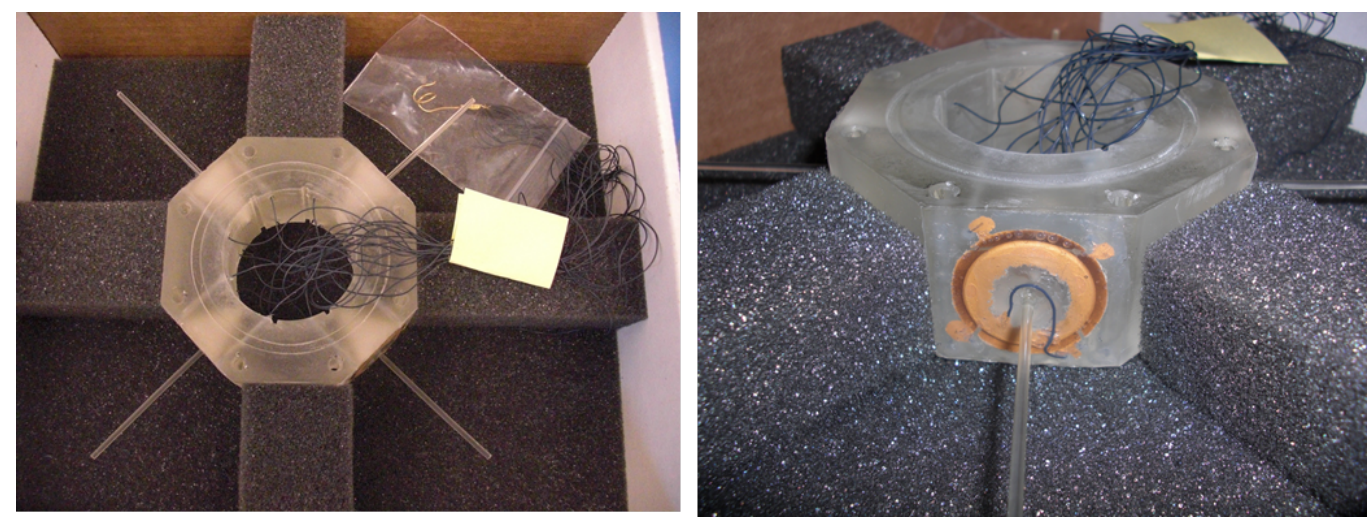

Figure 7.1: Ring array of whisker-like fluid motion sensors.

processor for the array. The WIDTS design must allow access to the PCB and battery without full disassembly, likewise the PCB needs to transmit the data collected without opening the WIDTS and risk water reaching the electronics. Throughout the design the separate components must be accessible for repair or replacement. The design therefore must consist of a nose, two ring arrays, and a means of attachment. Each component must form a waterproof seal with the adjacent components.

The preliminary design envisioned the WIDTS as having a torpedo shape $6 \mathrm{~cm}$ in diameter. The nose consisted of half an ellipsoid section with a long axis $12 \mathrm{~cm}$ in length. This was followed by a cylinder $6 \mathrm{~cm}$ in length for the sensor arrays followed by a tail section of another half ellipsoid with a long axis $28 \mathrm{~cm}$ in length. A plastic model in this shape was milled for evaluation. Flow testing in the water flume at $0.5 \mathrm{~m} / \mathrm{s}$ (Figure 7.2 showed a thin boundary layer with a small region of separated flow at the end of the tail section. Similar modeling in Ansys CFX using a shear stress transport model (SST) showed matching results with the boundary layer at the sensor location area in the $\mathrm{mm}$ range.

The whisker-like sensor is flat on the surface exposed to the fluid flow. Modifying the shape to be rounded would change the sensor performance by altering the symmetry along the two flow directions monitored and increase the construction complexity. As a result each sensor requires a minimum $3.4 \mathrm{~cm}$ by $3.4 \mathrm{~cm}$ surface to allow for the cone shape and still leave enough space to attach the PDMS membrane. This 


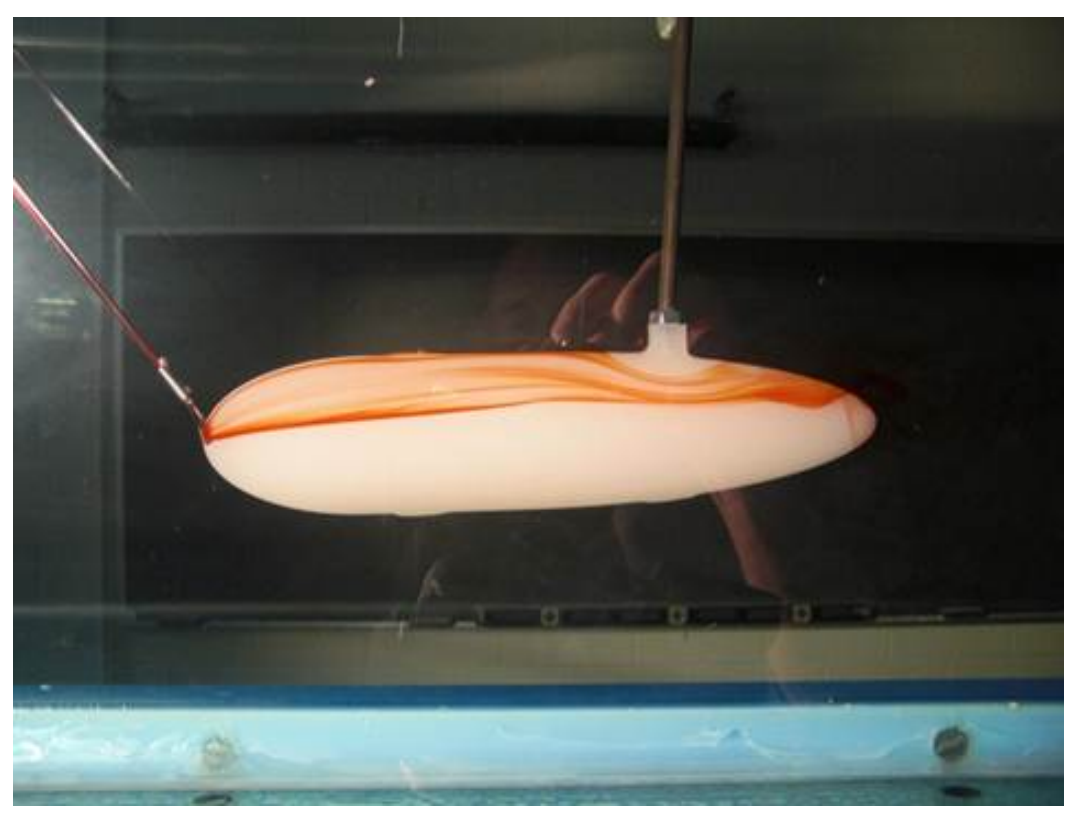

Figure 7.2: Torpedo shaped WIDTS in $0.5 \mathrm{~m} / \mathrm{s}$ flow with red dye showing water flow over the WIDTS surface.

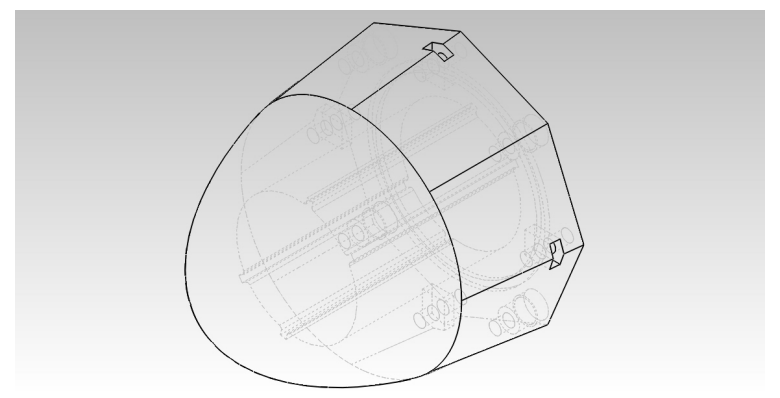

Figure 7.3: Modified ellipsoid nose section blending to the octagonal ring array.

requires the array rings to be octagonal in shape with a sensor on every other flat plate. The minimum diameter is thus $9 \mathrm{~cm}$. Having proven an ellipsoid body works as a nose section a similar approach was taken. The new nose is a half ellipsoid that blends into an octagonal base as shown in Figure 7.3 .

\subsubsection{Location and Attachment}

Several positions for the WIDTS location on Sprouts were considered. Working with the torpedo shape two options considered were above and below Sprouts' head. The left and middle pictures in Figure 7.4 show the torpedo simply attached with 

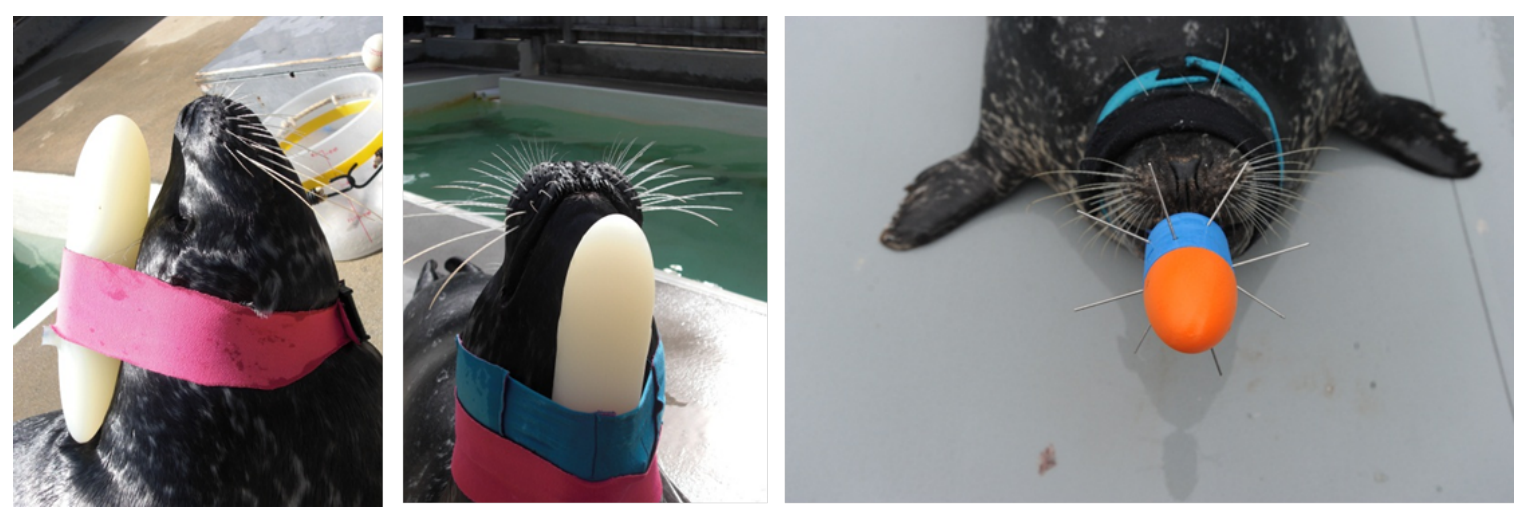

Figure 7.4: Possible WIDTS locations. WIDTS models attached to Sprouts on top of his head (left), below (middle), and held in his mouth (right).

a neoprene wrap to the harbor seal for evaluation. The placement of the WIDTS beneath Sprouts impeded his natural movement. When surfacing with his body in the vertical position, Sprouts bends his neck downward. This caused the WIDTS to strike his body and press it out of the wrap. Another issue with this location appeared when he completed a task and returned to the trainer at the pool wall. Sprouts surfaces near the wall and this position causes the WIDTS to scrape against the wall in a manner that would likely destroy the sensors.

Placing the WIDTS above Sprouts' head as shown in the left picture of Figure 7.4 caused no ill effects to the animal when swimming. He was comfortable with the added accessory and performed his tasks without distraction. Examining means of attaching the WIDTS for field trials with the arrays led to several issues. A hat shown in the left image of Figure 7.5 was modeled to match a harbor seal head and padded underneath. The WIDTS was to attach to the hat projecting it forward and aligning the sensors with Sprouts' vibrissae but roughly $10 \mathrm{~cm}$ above. The design proved very unstable while swimming. Sharp head turns characteristic when chasing an object caused the WIDTS model to move and become unseated. Object chasing near the surface also resulted in the WIDTS breaking the water surface in which case the sensors would not be monitoring any object wakes. In a similar fashion the offset meant the sensor arrays would not experience matching information experienced by 

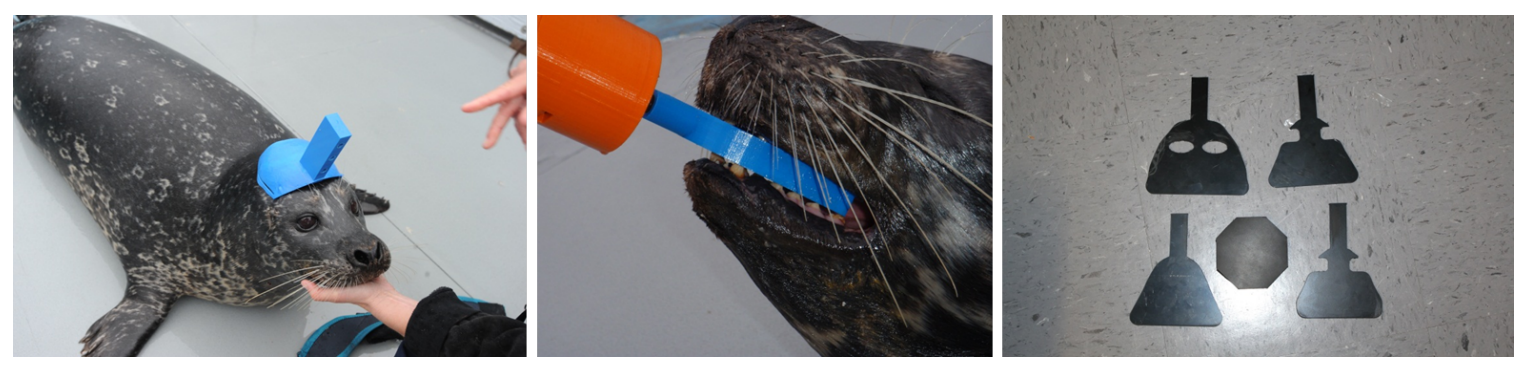

Figure 7.5: WIDTS attachment devices. Left: hat to locate WIDTS above Sprouts' head. Middle: early bite plate to hold WIDTS in front of Sprouts' muzzle. Right: bite plate variations.

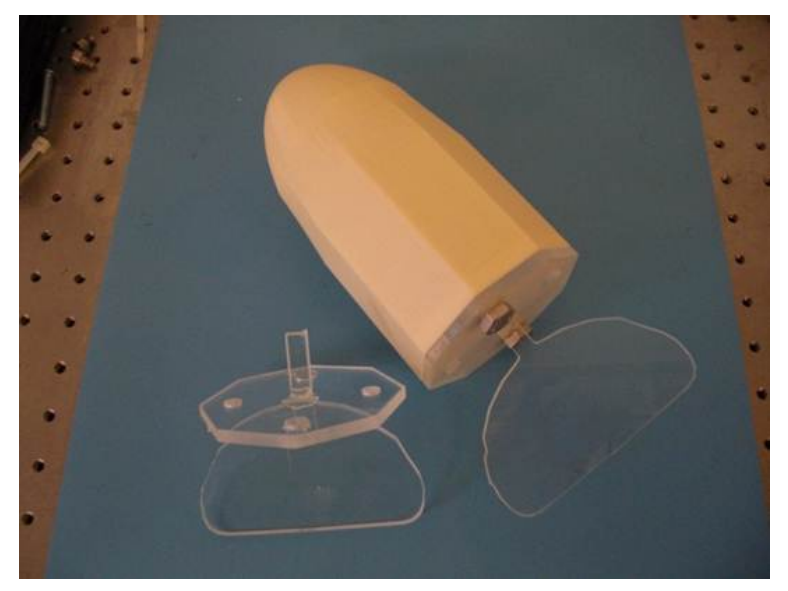

Figure 7.6: WIDTS mock up with finalized bite plates for training.

Sprouts.

Issues with the prior locations led to locating the WIDTS directly in front of Sprouts' nose as shown in the right picture of Figure 7.4. This insured the whiskerlike sensors experienced the flow information observed by the harbor seal. However it raised several new issues. The animal needed to be trained to bite down on an attachment device and carry the object while swimming. It also raised the possibility of the WIDTS blocking or disrupting the hydrodynamic signals allowing the harbor seal to track underwater objects. The interference issue is addressed in the next section. A test bite plate, made from a Dimension 3D printer, mimicking mouth guards worn by athletes (although constructed of hard plastic) was tested as shown in the middle of Figure 7.5 .

The raised edges of the mouth guard style bite plate irritated Sprouts. To resolve 
the issue several bite plate iterations were examined as shown in the right image of Figure 7.5. The designs look to accommodate the canine teeth of the animal and all raised areas are removed to avoid contact with the animal's gums. The end result was a bite plate nearly semi circular in design and $1 / 4$ in thick constructed of acrylic. The size just fits within the animal's mouth and allows the cheeks (where the vibrissae are located) to rest in their natural position. Only a $1 \mathrm{~cm}$ wide extension projects outward for attachment to the WIDTS. Figure 7.6 shows a full sized mock up of the WIDTS with the two final bite plates. One held the WIDTS $2 \mathrm{~cm}$ in front of Sprouts mouth while the other rested the back of the WIDTS against Sprouts nose.

\subsubsection{Design Evaluation}

As mentioned previously the location of the WIDTS directly in front of the harbor seal raises concerns regarding the system blocking or disrupting the fluid information allowing the animal to follow a moving object underwater. To examine the WIDTS effect, a model harbor seal head was constructed from CAT scan images of a juvenile harbor seal. The images sliced the head into 130 images. The outlines of the slices were mapped and compiled in AMIRA generating a 3D model of a harbor seal head. The model was then transferred to Solidworks to allow manipulation for fluid modeling in CFX and construction via 3D printing as shown in Figure 7.7.

Placing the seal head model in the flume, shown in Figure 7.8 with a WIDTS model, particle image velocimetry (PIV) measurements of the flow field around the models was conducted to verify flow simulations in Ansys CFX. Particle image velocimetry is a non-intrusive method for obtaining instantaneous fluid velocities within a projected laser sheet. For a review see [109 113].

In Figure 7.8 the PIV laser is shown underneath the water flume and the camera is level with the seal head looking into the flume from the side. In this arrangement the laser sheet shines vertically upward illuminating the flow field (seeded with hollow glass beads of 8-12 $\mu \mathrm{m}$ in diameter) on the left side of the seal head and WIDTS 


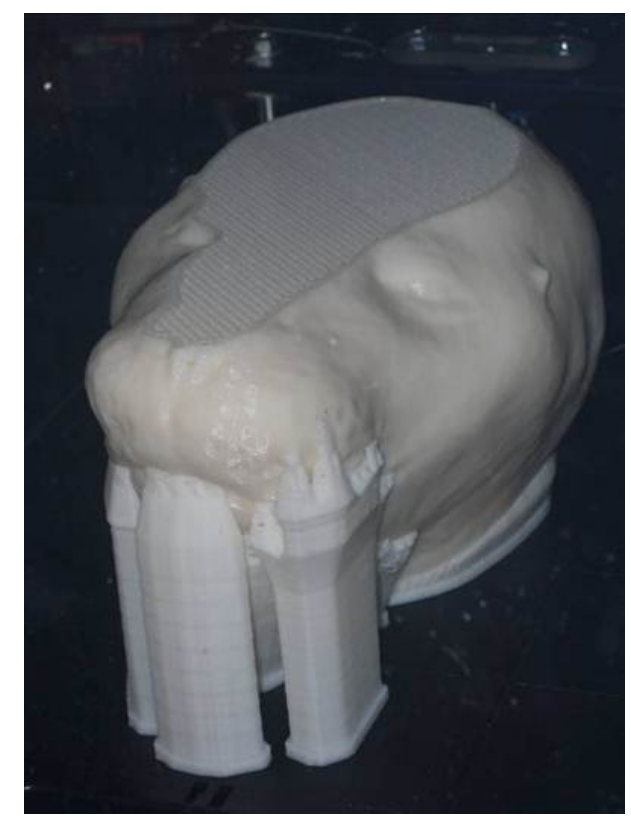

Figure 7.7: Harbor seal head modeling being printed.

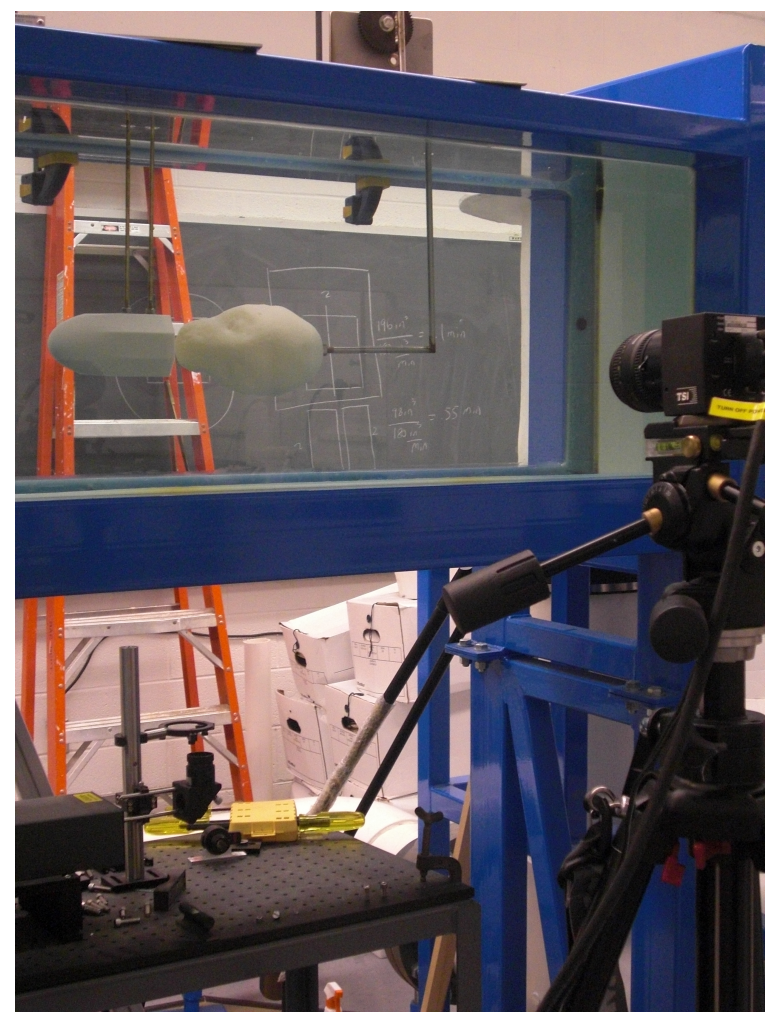

Figure 7.8: Harbor seal head with WIDTS for PIV testing. 


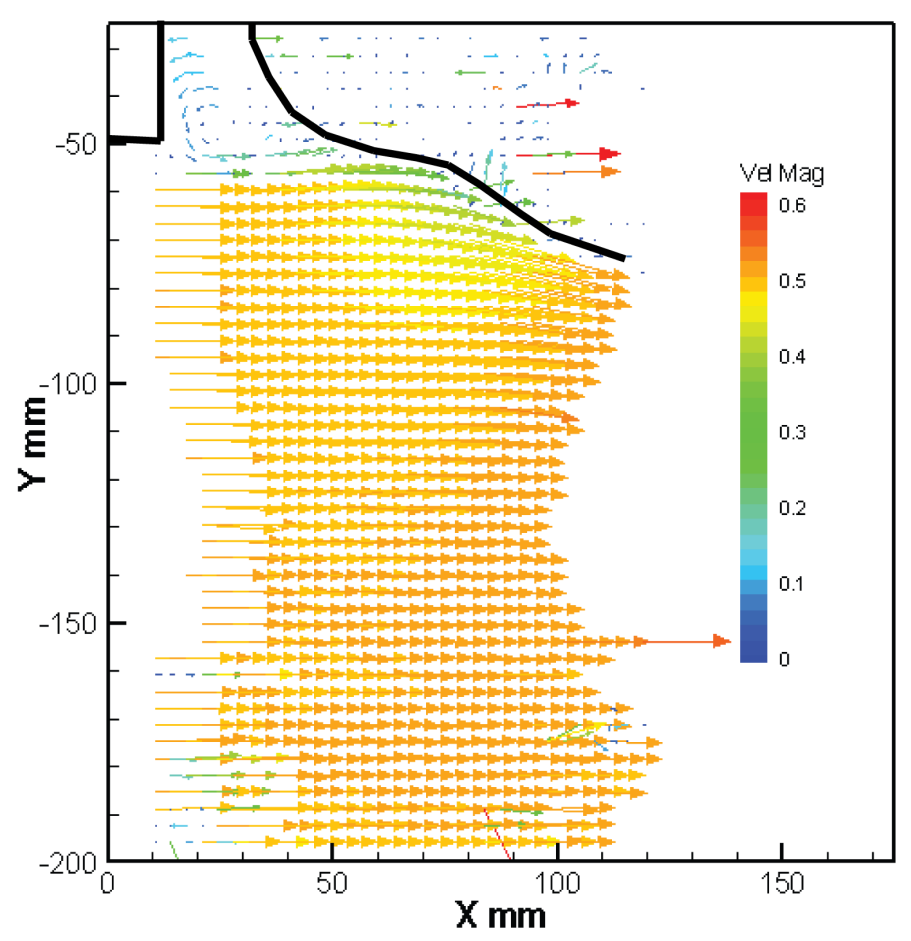

Figure 7.9: Average vector field from PIV measurement. Black outline indicates the edge of the seal head and corner of the WIDTS.

(crossing through the WIDTS mid plane). The camera snaps an image at each laser flash capturing the illuminated particles in the flow. This provides a snapshot of the flow field on the left side of the head and the space between the WIDTS and nose. The WIDTS is supported on the opposite side of the flow examined having no impact on the results. Likewise the harbor seal head is supported downstream of the flow by the sting. Of importance, all measurements and calculations are of a worst case scenario for the flow disruption by the WIDTS. The seal head was printed from a juvenile specimen resulting in a smaller head compared to the WIDTS than our test subject Sprouts. The final WIDTS placement with Sprouts also has the back of the WIDTS touching his nose effectively removing the majority of the space between the WIDTS and the head modeled here.

The averaged velocity vector field from 500 image pairs of a $0.5 \mathrm{~m} / \mathrm{s}$ flow around the seal head model and WIDTS is shown in Figure 7.9. The flow passes along the 
side of the WIDTS and continues nearly straight in the downstream direction until it encounters the seal head. There is weak region of slow recirculating fluid motion between the WIDTS and seal nose. The velocity field slows slightly around the area where the seal head grows just after the muzzle, but the vectors remain pointed in the downstream direction. The seal vibrissae should protrude out into the undisturbed flow.

The flume PIV tests were conducted to verify flow field modeling in Ansys CFX. For all simulations the shear stress turbulence (SST) model was used. The SST model developed by Menter [114] combines the robustness of the $k-\omega$ turbulence model near walls with the $k-\epsilon$ model away from the walls. Use of the SST model has shown to be in good agreement with experimental tests [115-117]. Modeling of cylinder wakes was conducted and shown to be in good agreement with experimental results verifying the SST model to capture the wakes of interest and indicating mesh densities required to capture the flow physics. Boundary conditions of the simulations included:

- prescribed inlet velocity

- free shear along walls

- zero velocity on body surfaces

- zero averaged pressure over the outlet

The simulations modeled the flow in a rectangular prism $42 \mathrm{~cm}$ in length. The seal head was located at the outlet $32 \times 32 \mathrm{~cm}$ in cross section. All surfaces incorporated inflation meshing and in the case of the cylinder wake, the mesh density was increased in the wake regime resulting in a total of 10108000 elements. Prior validation of cylinder wakes proved this density to be adequate in capturing the wake and resolving the flow. The inlet flow speed was $0.5 \mathrm{~m} / \mathrm{s}$ for all cases. The velocity contour of the mid plane passing through the harbor seal head with and without the WIDTS present are shown in Figure 7.10 for steady state flow. The simulation matches the results 

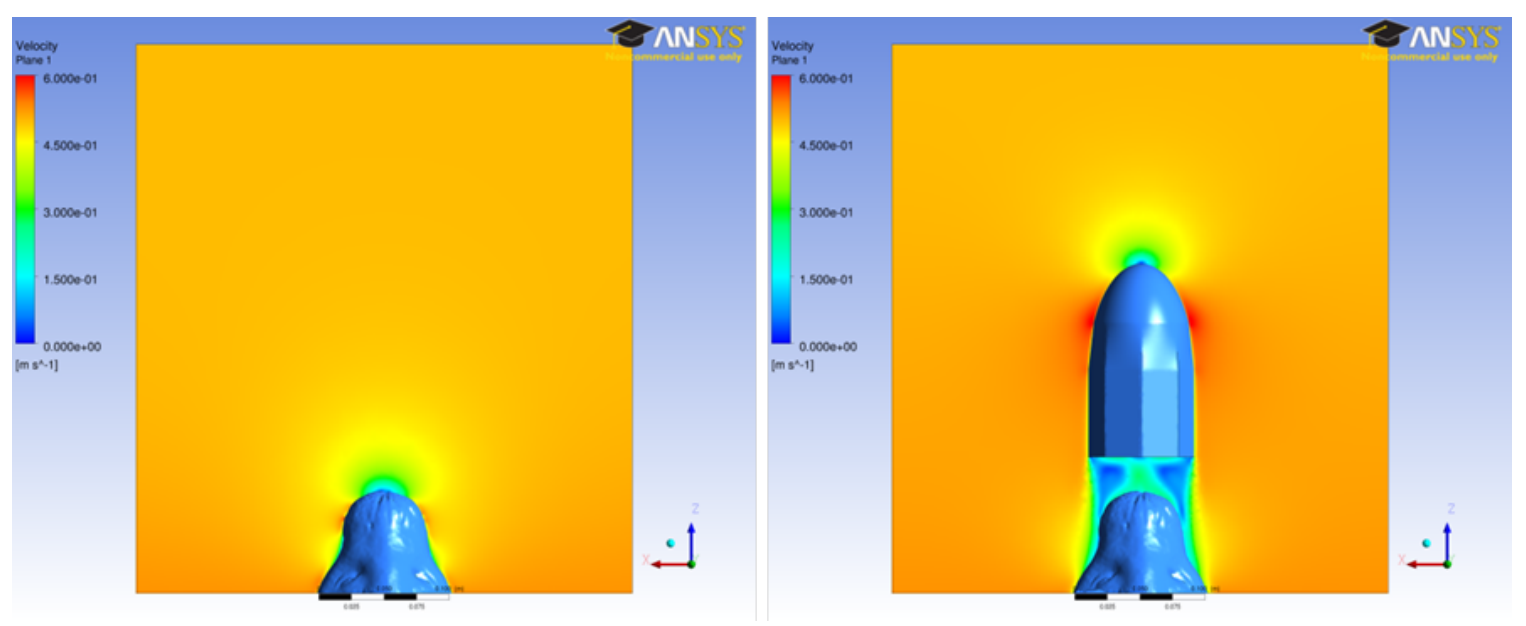

Figure 7.10: Steady state velocity contour plot of $0.5 \mathrm{~m} / \mathrm{s}$ flow around seal head with and without WIDTS.

captured by PIV in the flume. There is an area of low speed recirculation between the WIDTS and seal nose. The fluid motion passed along the WIDTS in the downstream direction until turning when it encounters the head.

Taking a closer look at the area around the seal head and between the nose and WIDTS, the velocity vectors colored for velocity magnitude are shown in Figure 7.11. Again the area of recirculation is evident between the WIDTS and harbor seal head. The flow is also significantly slowed around the seal muzzle in the region vibrissae are present compared to the case without the WIDTS. Of importance is the point that the vectors indicate the flow is still moving downstream in the vibrissae region despite the WIDTS obstruction. There is little difference in the flow field a short distance away from the head in the area into which the whiskers extend. It appears the vibrissae should still be capable of reaching the free stream flow and capturing fluid motions.

Placing a $2 \mathrm{~cm}$ diameter cylinder $10 \mathrm{~cm}$ directly upstream of the WIDTS or 27 $\mathrm{cm}$ upstream of the seal's nose, the transient case is simulated with and without the WIDTS in front of the harbor seal head. Time steps of $0.01 \mathrm{~s}$ were used with ten iterations of the governing equations between time steps. To compare the wake observed by the pinniped vibrissae with and without the WIDTS two data points, 

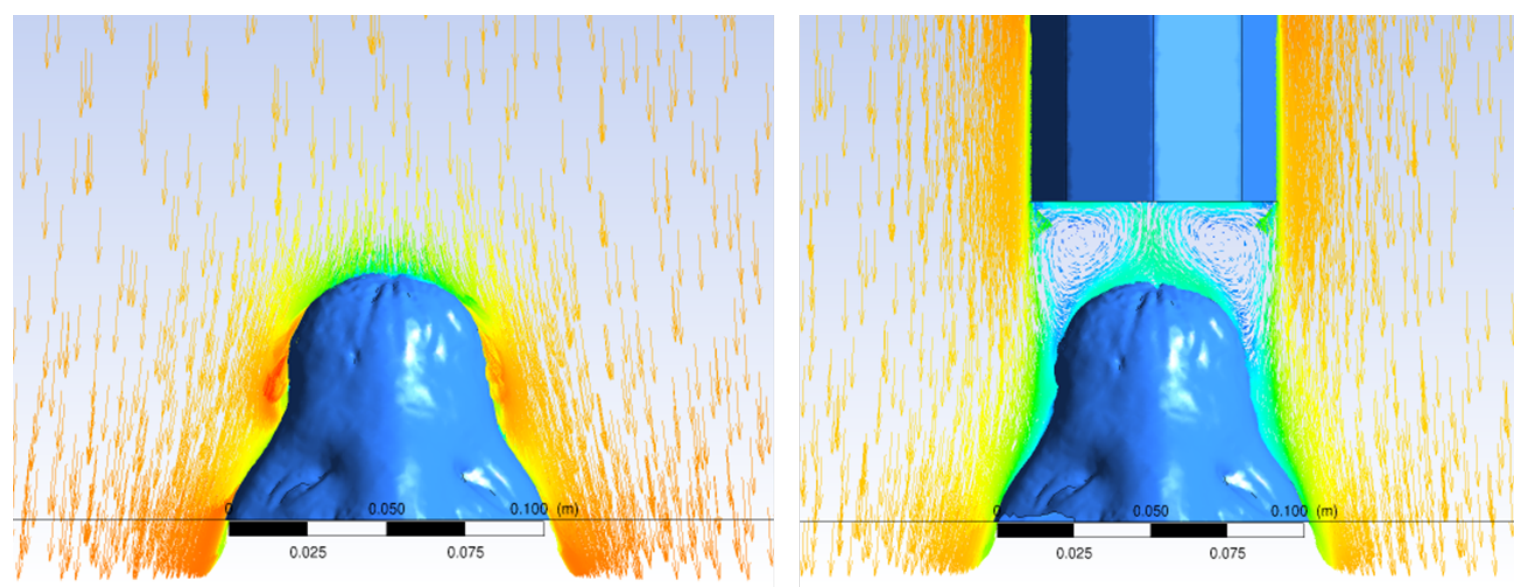

Figure 7.11: Velocity vectors of $0.5 \mathrm{~m} / \mathrm{s}$ steady state flow around seal head with and without WIDTS.

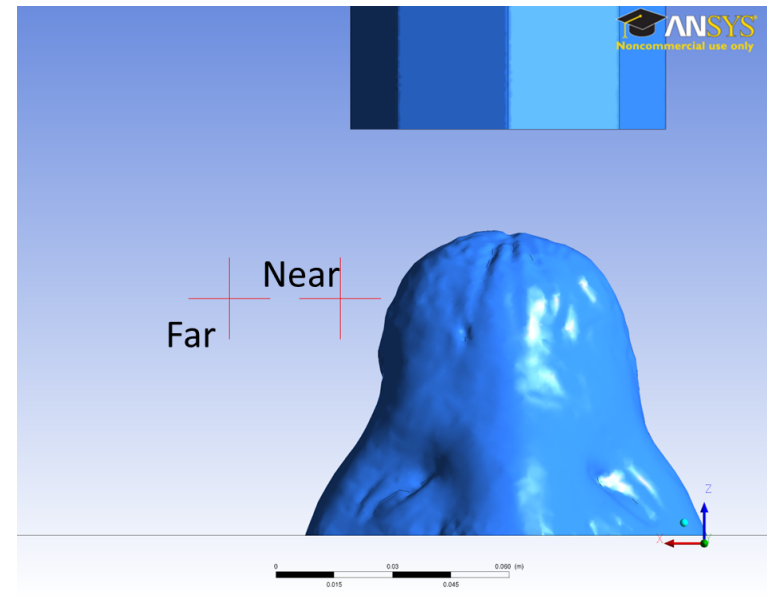

Figure 7.12: Location of near and far data points monitoring cylinder wake flow.

near and far shown in Figure 7.12, monitored the results. These points were chosen to be located at the edge of the flow slowed by the WIDTS and further out, but still within vibrissae lengths.

Figure 7.13 shows the resulting velocity magnitudes at the near and far observation locations. Without the WIDTS obstructing the flow observed by the seal (left plot) the far location shows a consistent velocity with an oscillation about $0.52 \mathrm{~m} / \mathrm{s}$. The near location without the WIDTS contains both a higher frequency oscillation and a larger velocity magnitude range with an average lower than that of the far point. With the WIDTS obstructing the wake, the far location's velocity magnitude 

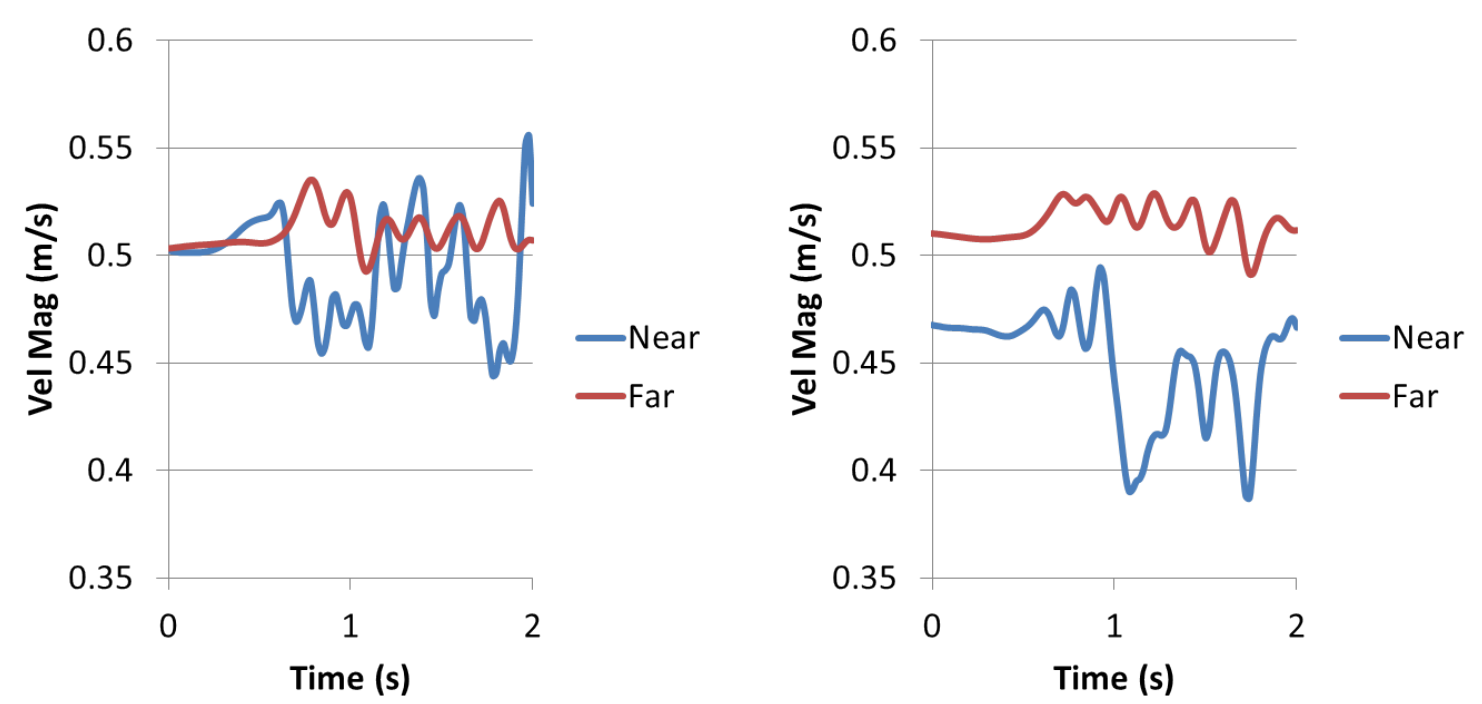

Figure 7.13: Velocity magnitude monitored by the near and far locations in the presence of a cylinder wake with (right) and without (left) the WIDTS obstructing the flow ahead of the harbor seal head.

is very similar to that without the WIDTS at the same point. There is a significant difference in the velocity observed by the vibrissae at the near location with the WIDTS obstruction. The magnitude is nearly $20 \%$ lower and loses some of the higher frequency information.

The vibrissae act as cantilever beams with an end fixed at the seal. Therefore, forces acting further out along the whisker have a greater influence on the whisker's deflection. As a result the far field velocity magnitude is likely to play a larger role in the information gathered by the seal than that of the near location point.

Taking a Hanning window of the frequency response of the velocity magnitude at each location indicates the harbor seal should still be capable of detecting the cylinder wake in spite of the WIDTS obstructing the flow. The predicted shedding frequency of the cylinder under the prescribed flow condition is $5 \mathrm{~Hz}$. In both cases the vibrissae experience a $5 \mathrm{~Hz}$ signal and this signal is observed at both the near and far locations. The presence of the WIDTS reduces the amplitude of the $5 \mathrm{~Hz}$ signal but it remains well within the observable range of harbor seals.

The results presented here are for a single wake instance. Varying the flow con- 

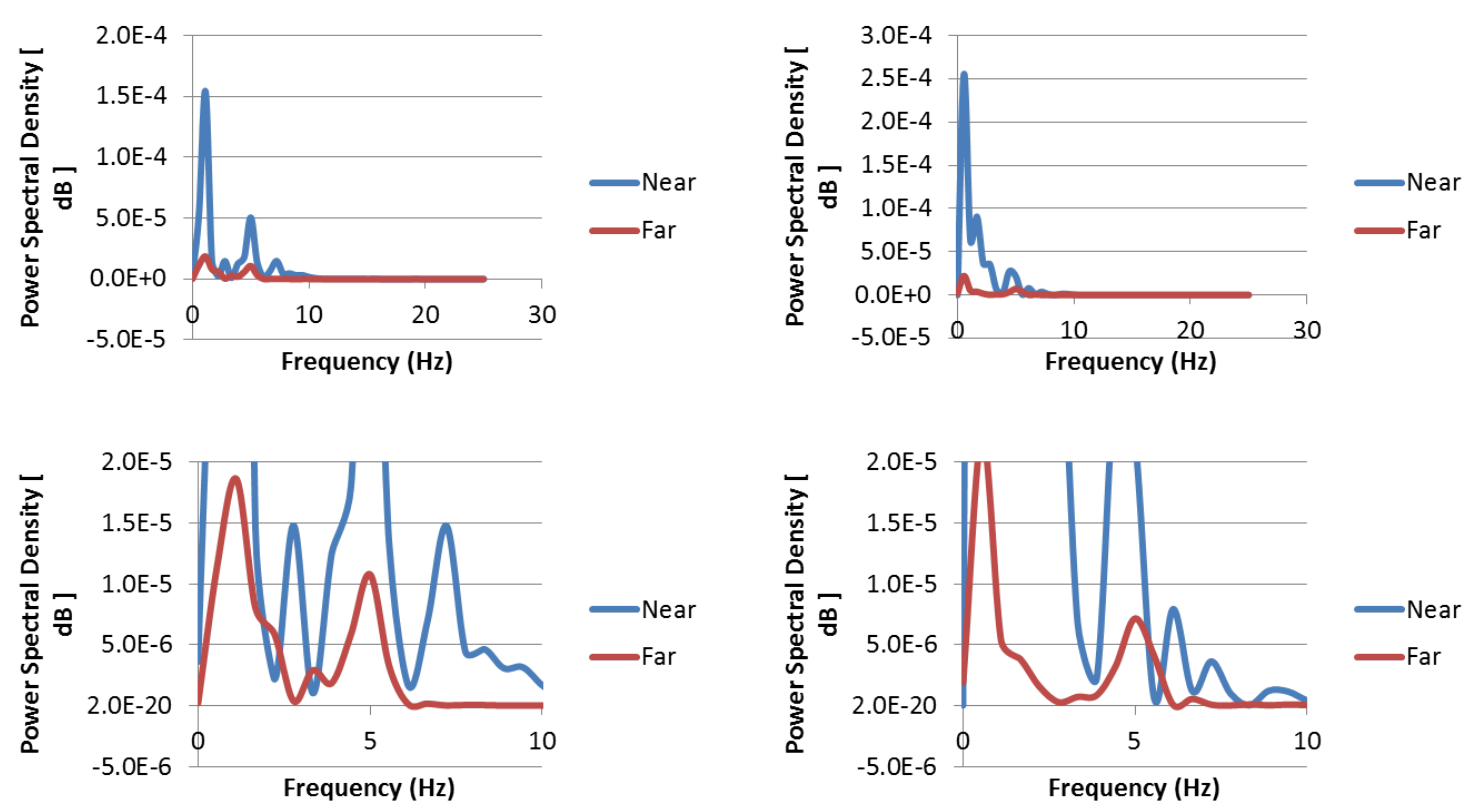

Figure 7.14: Frequency response of velocity monitored by near and far locations in the presence of a cylinder wake with (right) and without (left) the WIDTS obstructing the flow ahead of the harbor seal head.

ditions and cylinder size result in similar results (with larger cylinders generating wider wakes and even better frequency response with the WIDTS obstruction). It is worth repeating that the effects from the WIDTS will be mitigated by larger harbor seal head and reducing the space between the nose and WIDTS as in the case for real world tests. Indeed Sprouts proved capable of tracking underwater objects while carrying the WIDTS mock up.

\subsubsection{Construction}

Proving the WIDTS dimensions still allow the harbor seal to track hydrodynamic signals, all the prior design parameters must be accommodated into the WIDTS construction. The WIDTS itself consists of the nose section, two ring arrays, and the back plate attached to the bite plate. Like the bite plate, the back plate was constructed from 1/4 inch clear acrylic. This provided a viewing window into the WIDTS to check for leaks during tests. The o-rings set into grooves on each section 


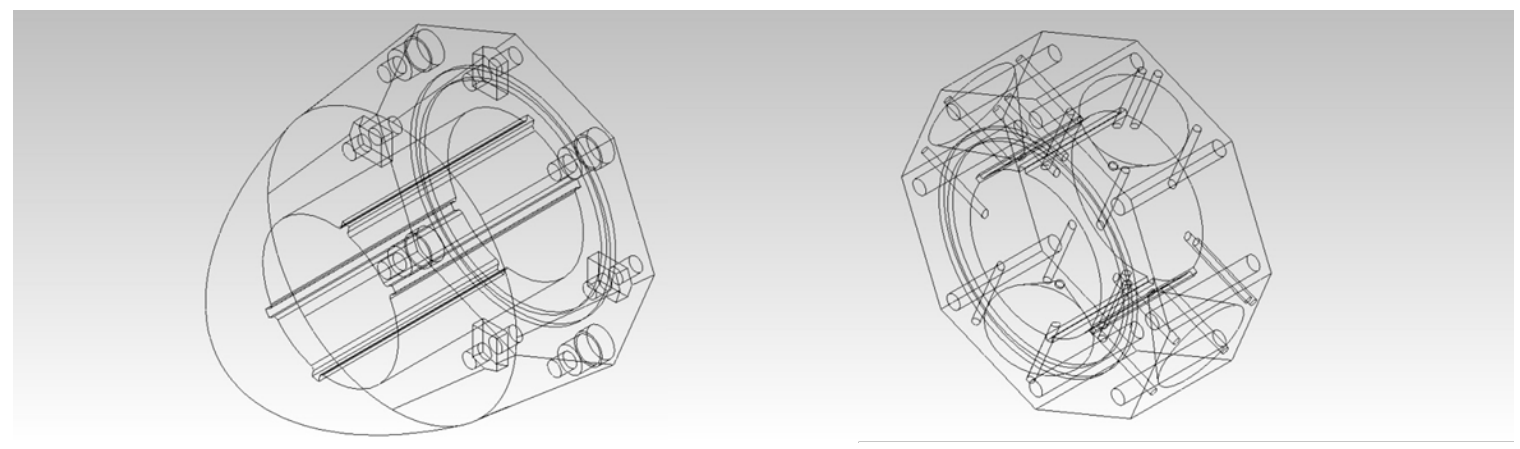

Figure 7.15: Wire frame schematic of the nose section (left) and a sensor array ring (right).

waterproofed the WIDTS.

As was shown in Figure 7.1 each ring array contains four sensors on every other side of the octagon. Five $2 \mathrm{~mm}$ holes surrounding the sensor were pathways for the wires to attach from the PCB to the capacitance plates. Accounting for the size of the outer sensor cone penetrating into the ring, the maximum hollow space within the WIDTS was $4.4 \mathrm{~cm}$ in diameter. Slots on opposite sides of the hollow space allowed the PCB to slide into the array and lock into place. A second set of slots was offset by 45 degrees allowing the slots to align in any ring orientation. A wire frame schematic of the array design is shown on the right of Figure 7.15. The ring arrays were constructed from molded epoxy and manufactured by Micro System Inc.

The nose section is shown on the left of Figure 7.15 . The hollow cavity and PCB slots extend $6 \mathrm{~cm}$ into the nose. This provides a maximum space for the PCB of 12.8 by $4.4 \mathrm{~cm}$. The nose was manufactured on a Dimension 3D printer and coated with fiberglass resin to seal the printed layers. The WIDTS sections are held together by eight $3 \mathrm{~mm}$ diameter threaded rods extending from the back plate into the nose section and locked into place on each end by a washer and nut. The eight rods pass through $4.4 \mathrm{~mm}$ holes located at the octagon corners of each section. Four of the rods bolt together holding the back plate and arrays firmly together. The final four rods bolt from the back plate to the nose section. In this manner four rods can be removed to allow removal of the nose section and provide access to the electronics 


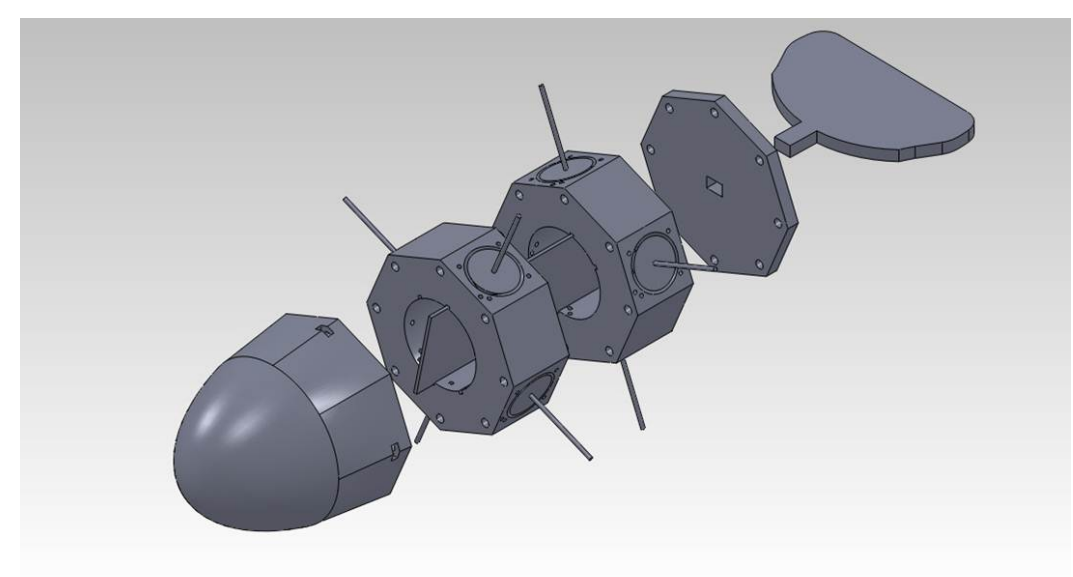

Figure 7.16: Exploded view of the final WIDTS assembly.

without fully disassembling the WIDTS. Figure 7.16 provides an exploded view of all the WIDTS sections for assembly.

\subsubsection{Data Collection}

The electronics secured within the WIDTS complete the sensor circuit, acquire analog data, peak detect the sensor output, digitize it, and store the data to memory. The PCB is powered by a Route JD model PD3048 battery providing roughly 3 hours of life. The PCB excites each sensor with a 3.7 V $790 \mathrm{~Hz}$ sinusoidal signal and measures the 8 sensors' responses on each quadrant across a $1000 \Omega$ load resistor. To avoid false data input of whisker rotations caused by the Sprouts shaking his head accelerometers and gyroscopes mounted to the PCB monitored the WIDTS orientation and motion. Data was transfered from the PCB to computer via bluetooth. The PCB was designed by Yousef Shahkhsheer and its components were installed by $3 \mathrm{~W}$. The software was programmed in Java by Chris Gregg.

\subsection{Chase Object}

A Thunder Tiger Neptune remote controlled (RC) submarine was selected as the chase object for Sprouts shown in Figure 7.17. The Neptune is $77.4 \mathrm{~cm}$ in length and 


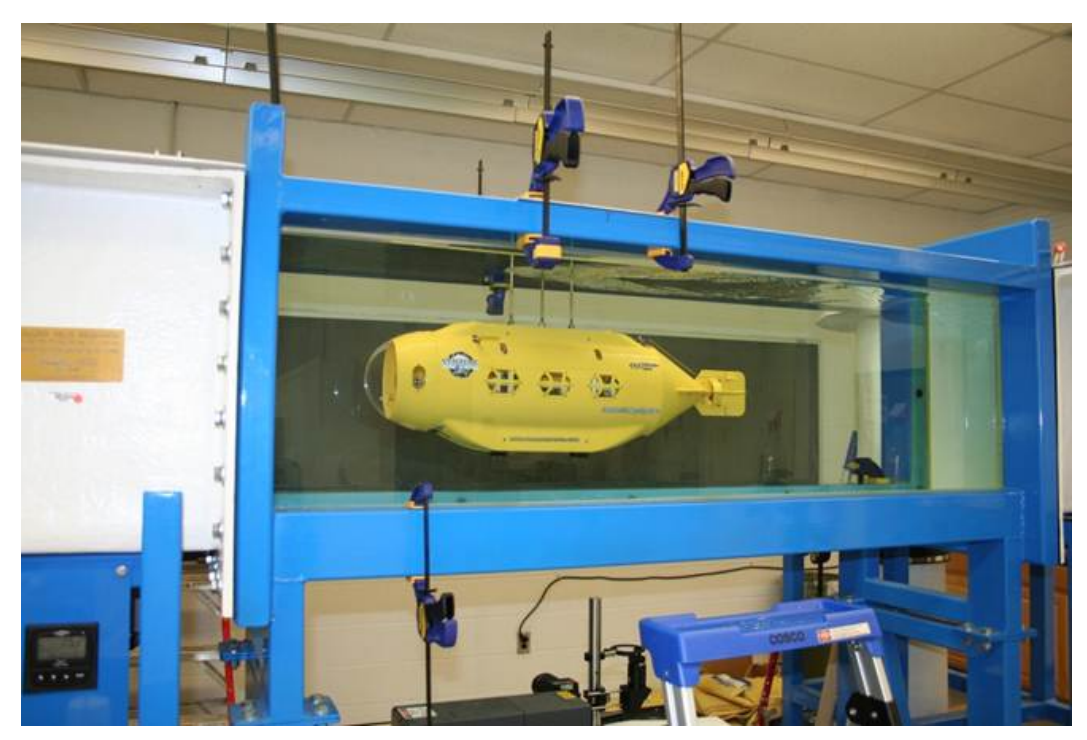

Figure 7.17: Chase vehicle in flume.

$29 \mathrm{~cm}$ in diameter. The RC submarine has a top speed of $0.56 \mathrm{~m} / \mathrm{s}$ and depth limit of $5 \mathrm{~m}$. To accommodate use in salt water the sub antenna was extended out from the body and attached to a float forcing the antenna to extend out of the salt water when in use. Testing in the water flume was conducted at the submarines top speed verified before securing the sub for PIV testing (located at a prescribed flume speed of $41.7 \mathrm{~cm} / \mathrm{s}$ ). PIV measurements where conducted in 5 sections of 18 by $30 \mathrm{~cm} .500$ image pairs were taken and the sub was moved forward by $15 \mathrm{~cm}$ to capture the flow of the next section. In this manner the flow field $70 \mathrm{~cm}$ behind the sub was captured with greater resolution.

The wake behind the self propelled submarine is shown in Figure 7.18. The size of the sub campared to the cross sectional area of the flume generates significant distortion of the wake. Although the flume was running at $41.7 \mathrm{~cm} / \mathrm{s}$ the free stream velocity is actually closer to $47 \mathrm{~cm} / \mathrm{s}$. The bottom wall of the tank forces the lower propelled water to actually move upward indicating the walls are impacting the observed wake. The dark blue area on the left of the plot is the submarine body. The contour plot shows the velocity magnitude through the midplane of the submersible. The two regions of incleased flow speed directly behind the sub are thought to actually 


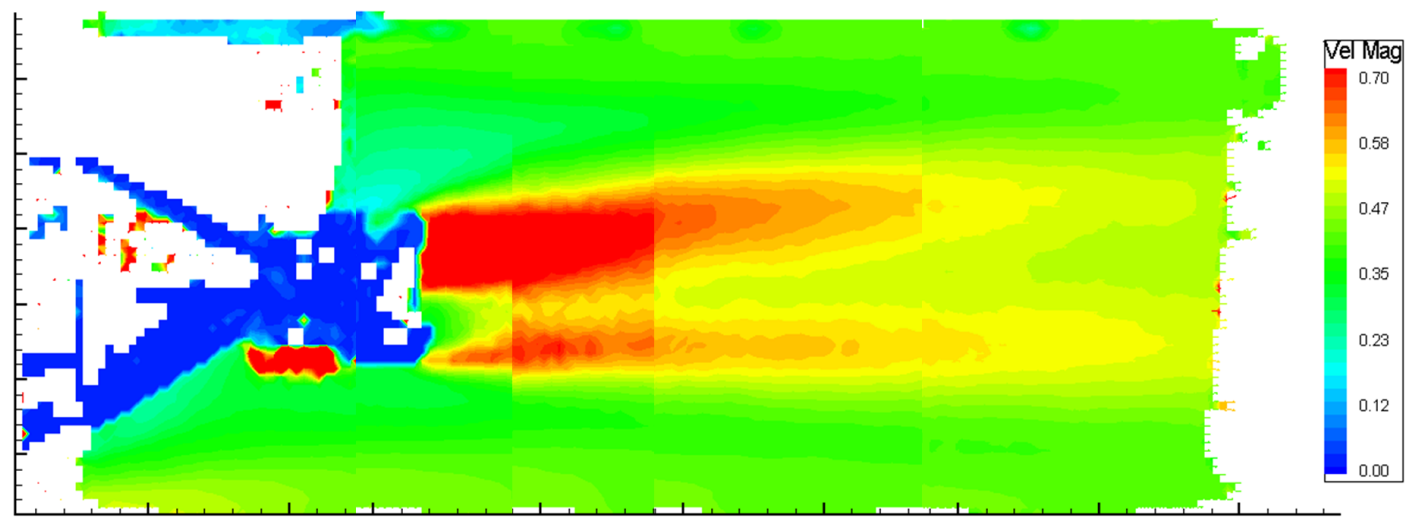

Figure 7.18: Contour plot of averaged flow field behind Neptune submarine.

be part of a single propeller driven jet flow. PIV can only capture the fluid motion in two directions. The spinning propeller is rotating the flow as it forces the water back. The out of plane motion registers as low flow velocity. The PIV measurements are a rough image of the flow field that will be experienced by the WIDTS and Sprouts. It indicates the wake should cover an area at least as large as the sub's diameter and the wake is likely to persist for several body lengths with measureable velocities.

\subsection{WIDTS Field Trials}

The focus of this chapter is the WIDTS design and development. This section presents preliminary results of field trials showing an example of test results of the deployment of the WIDTS on Sprouts chasing the RC submarine.

\subsubsection{Experimental Methods}

Training of the harbor seal, Sprouts, was conducted by Dr. Colleen Reichmuth's group at the Long Marine Lab at the University of California Santa Cruz. Sprouts was blindfolded, given the WIDTS, and held in a wait position with his head out of the water as the submarine began to travel around the pool. Sprouts was then released. 


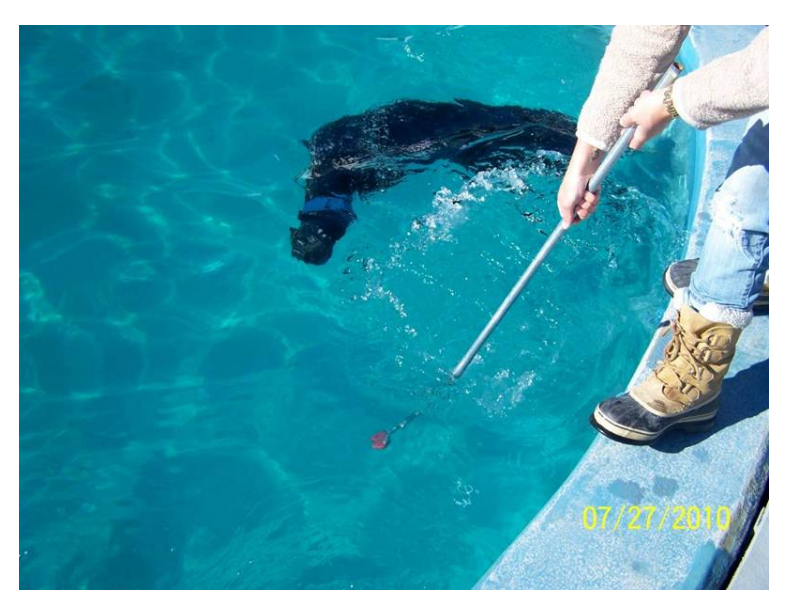

Figure 7.19: Blindfolded Sprouts chasing a $6 \mathrm{~cm}$ ball during training.
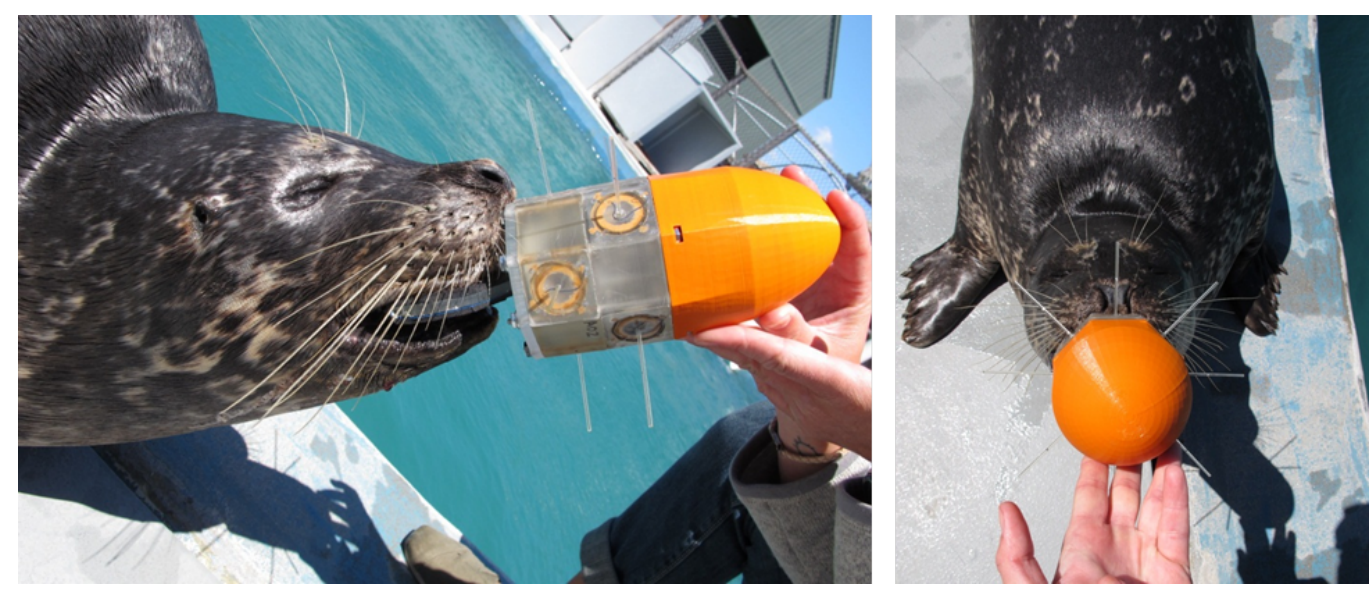

Figure 7.20: Side and front view of Sprouts holding WIDTS for testing.

He had to locate the sub wake, catch up to the model, and then chase it remaining about $1 \mathrm{~m}$ behind the submarine. The only information about the location of the sub received by Sprouts was wake hydrodynamic signals picked up by his vibrissae.

After each run the WIDTS was collected from Sprouts and the data transferred via Bluetooth before clearing the memory and returning the WIDTS to Sprouts for another run. Video of both the submarine's and Sprouts' paths were monitored by a stationary camera over the pool recording a top view of the each test session. A GoPro underwater camera was manipulated by a trainer to follow the sub and Sprouts around, providing a side view of the chase. Tracking of the submarine and the WIDTS was conducted in Matlab through a code provided by Michael Landau. 

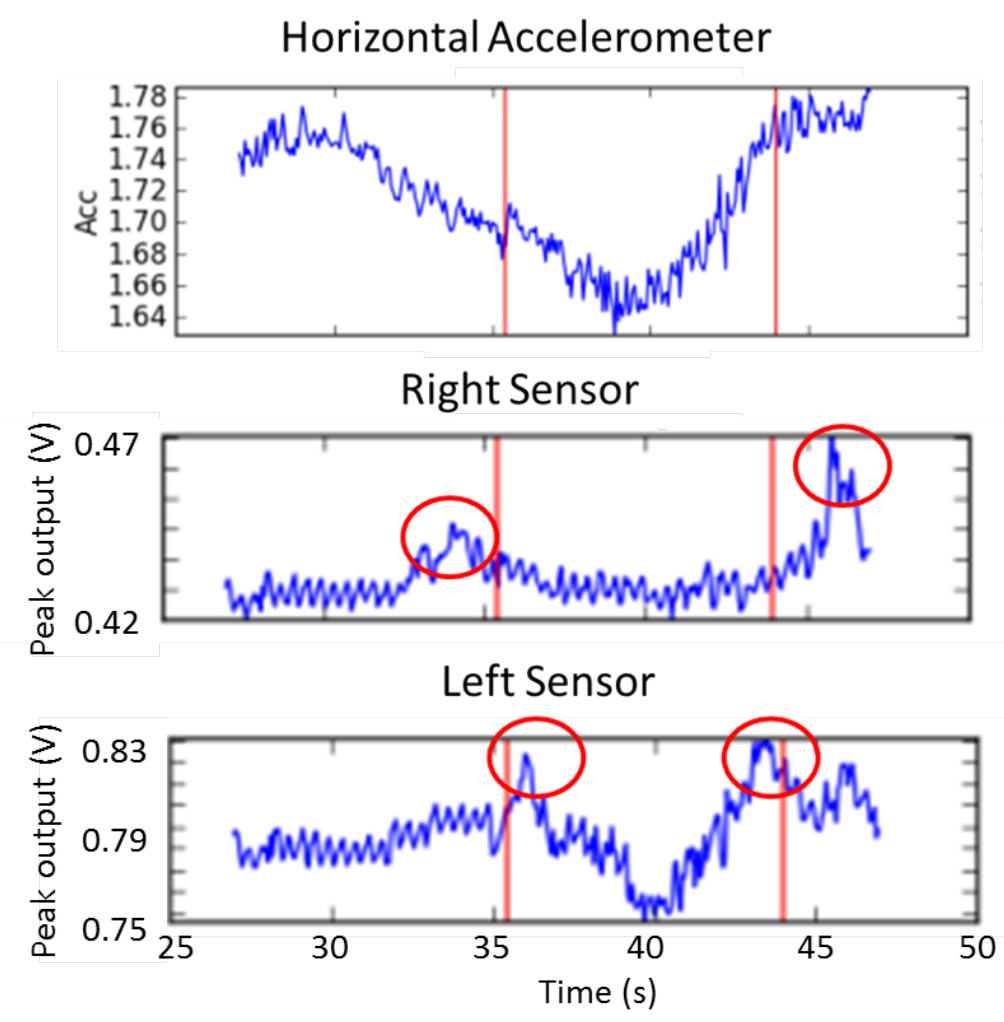

Figure 7.21: Data from sweeping WIDTS behind RC submarine. Red lines indicate when the WIDTS was directly behind the sub. Top plot shows the WIDTS motion, middle and bottom plots show rear quadrant data for the right and left sensors with circles around wake detection spikes.

The tracking code detected the colors of the WIDTS and sub and plotted the center of mass location of the the objects as they traveled around the pool.

\subsubsection{RC Submarine Detection}

To verify the WIDTS ability to detect the sub a simple test held the sub stationary under power. The WIDTS was manually swept across the sub's wake $0.5 \mathrm{~m}$ behind the propeller and back thus crossing the wake twice. Overhead video recording showed the WIDTS was directly behind the submarine at the red lines in Figure 7.21. The top plot of the accelerometer data shows the WIDTS traveling to the right, then reversing directions between the red lines and returning back to the left. This sweeping of the WIDTS behind the RC submersible caused the sensors in the arrays to detect the 
wake at offsetting times. Looking at sensors from opposite sides of the WIDTS (right and left sides), two distinct spikes in the data are observed in each set of the quadrants facing the sub. The wake strikes the right side sensor first followed by the left side. The opposite occurs on the return sweep, striking the left sensor then the right. The wake is detected by peaks in the data circled in Figure 7.21.

\subsubsection{RC Submarine Tracking}

The WIDTS and submersible were tracked during chase testing. The tracking plots the center of the objects outline. In all tests Sprouts closely followed the sub track. A weaving in and out of the sub path is observed in several tracks and assumed to be Sprouts moving in and out of the wake for detection purposes. A sample test path is shown in Figure 7.22, The tracks indicate Sprouts is able to closely follow the exact submarine path. Some of the weaving in and out of the wake is observed in the lower half of the figure where the red track line oscillates back and forth. From the tracks Sprouts appears to have a preference to stay just on the outside of the submarine track. However the yellow path plots the center of the submarine's location and not the wake location. Examining the orientation of the submarine in Figure 7.22 the yellow path ends directly on the sub however the tail is pointed outward from the curvature of the track and the propeller displaces water in this direction (on the outside of the tracked path). It is assumed that the outer path taken by Sprouts is actually along the wake trail left by the submarine.

As an example of the potential data available from the tests, two instances of the chase are shown in Figure 7.23 corresponding to 27.2 and 29 seconds into the test. Here the submarine has started its track and Sprouts is released to find the vehicle. The red circles in the images indicate the area crossed by the submarine and therefore the area of the residual wake.

Again examining the left and right sensors on the WIDTS (Figure 7.24), the two time instances from the previous snapshots are marked with red lines. These indicate 


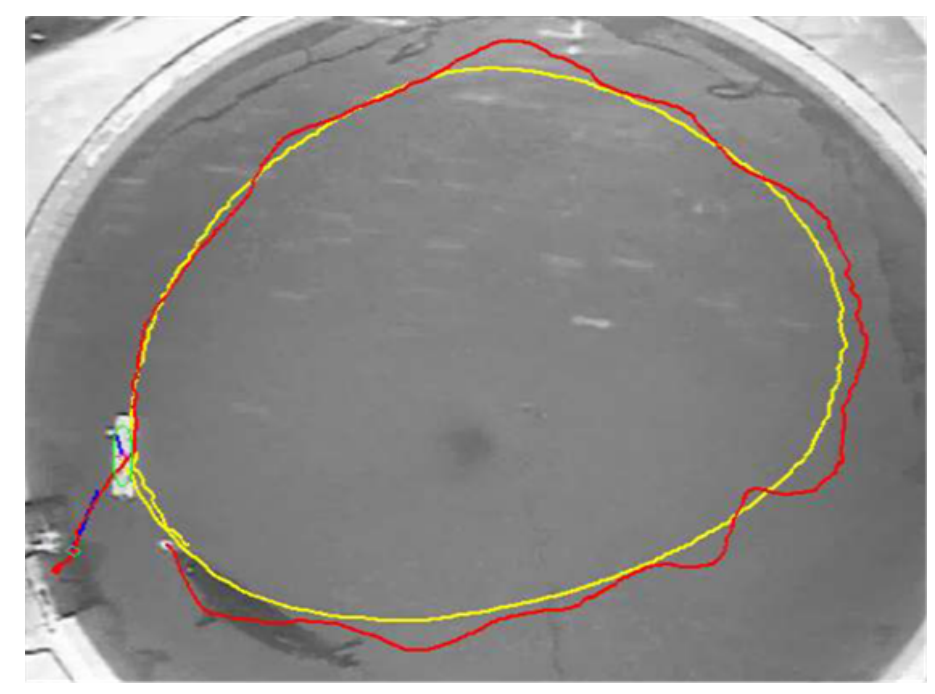

Figure 7.22: Track of the sub (yellow) and path of the WIDTS held in Sprouts' mouth (red).

\section{$27.2 \mathrm{~s}$}

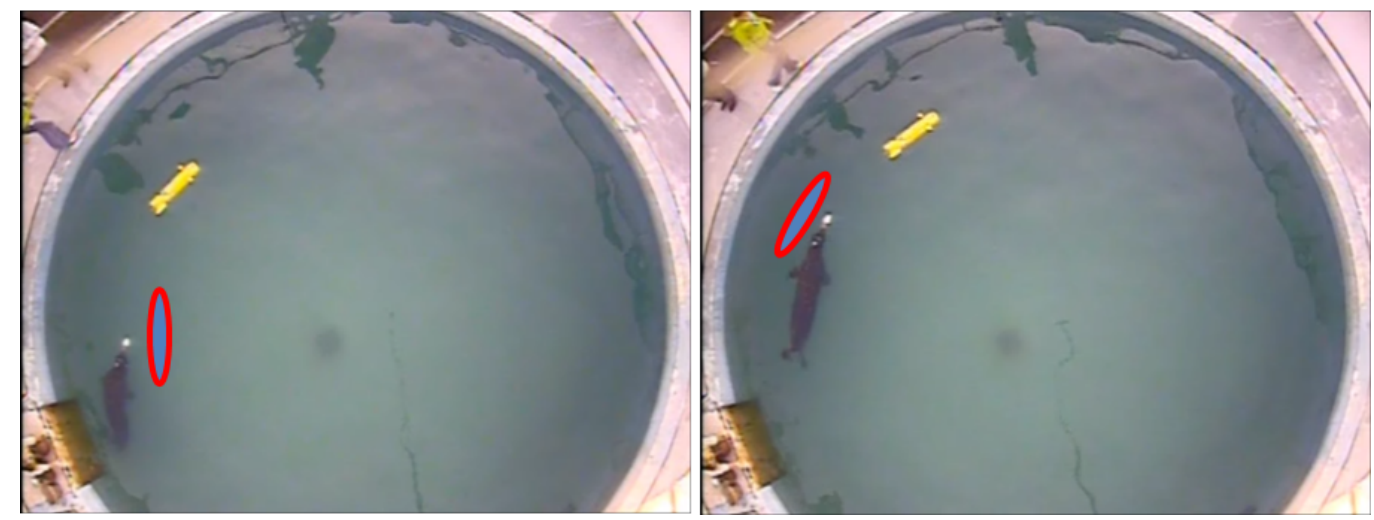

Figure 7.23: Snapshots of Sprouts chasing the sub at 27.2 seconds (left) and 29 seconds (right). The residual submarine wake location is circled in red. 


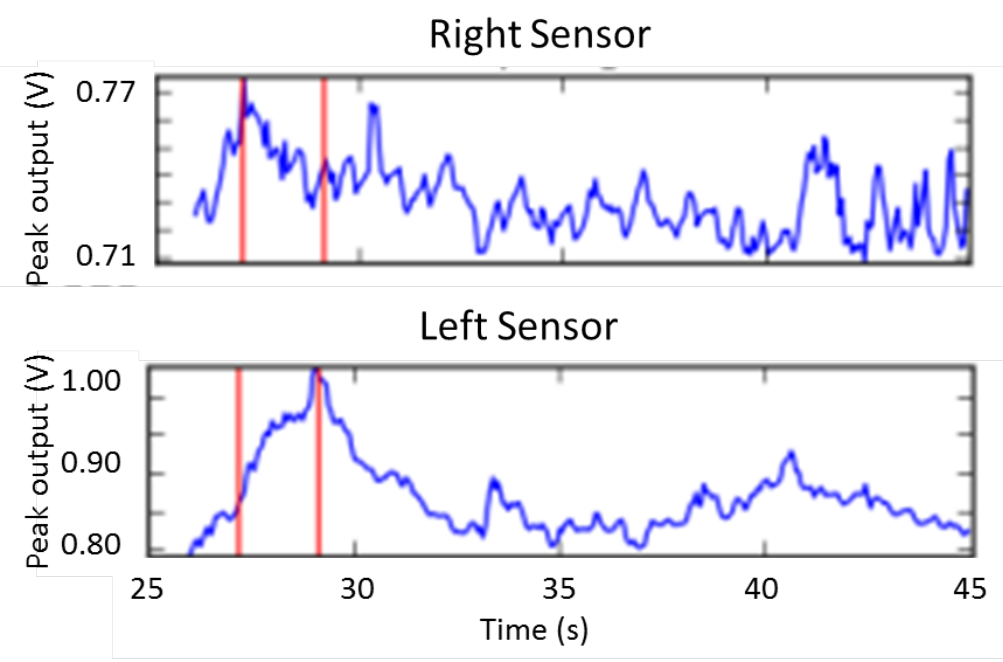

Figure 7.24: Left and right sensor data with red lines marking peaks indicating submarine wake detection.

two sides of the WIDTS entering and exiting the wake laid down by the submersible. A spike is shown on the right sensor (first red line) followed by one on the left sensor (second red line). These occur shortly after Sprouts is released and the sub has passed by the right side of Sprouts. The WIDTS initially catches the wake on right side then the left side.

\subsection{Discussion}

Examining the design requirements imposed by the cone-in-cone sensor and locations available for attachment to a harbor seal, the final WIDTS starts as an ellipse transitioning into an octagonal cylinder ending with a bite plate to be carried by Sprouts. The location insured the sensor arrays passed through the same volume of water as the sensory system of Sprouts. This design was shown to impact the pinniped's vibrissae of which it was placed in front, but not to an extent causing the the seal to lose its ability to track hydrodynamic signals. A self propelled RC submarine was selected as the chase vehicle. The top speed of roughly $0.5 \mathrm{~m} / \mathrm{s}$ is less than the normal swimming speed of harbor seals, but Sprouts was trained to slow his swim and 
maintain a follow distance of about $1 \mathrm{~m}$ behind the propeller. Initial testing of the WIDTS with the sub showed it could pick up the propeller driven flow and indicate the side of the WIDTS experiencing the wake. This shows potential of the WIDTS as a wake tracking system.

The sub testing in the flume does provide an idea of the wake behind the RC submarine, however the model is large compared to the flume size. The diameter of the submarine blocks $37.8 \%$ of the flume cross sectional area. This likely causes some differences between the recorded wake and that of the actual tests with Sprouts. The water flow must accelerate around the model increasing the free stream flow and the width of the wake is likely constricted.

Several issues arose throughout the WIDTS testing. Field testing required a cooperative animal (the WIDTS was dropped and damaged on one run). The waterproofing proofing needs improvement. The ring arrays had uneven surfaces at the o-ring interfaces causing slow leaks in the WIDTS during testing. Flooding of the WIDTS resulted in the loss of one PCB. The data collected on the PCB was also under sampled causing aliasing. The sensors were excited at $790 \mathrm{~Hz}$ but while recording all 32 quadrants, 3 gyroscopes, and 3 accelerometers the data was only recorded at 16 Hz. The result of this under sampling is the sharp oscillations observed in the data as shown in Figure 7.21. This was originally attributed to noise but later deemed a result of aliasing. Another issue discovered during testing was blackening of the gold plated capacitor plates. This had never occurred until the PCB was used to excite the sensors. The noticeable change in plate color occurred in as little as five minutes under power from the PCB. The blackening appears to raise the noise in the sensor output, combining with the aliasing issue previously mentioned. It is hypothesized this may be caused by higher currents passing through the sensor than when excited with the function generator. 


\subsection{Summary}

The goal of this chapter was to develop a self contained package for the sensors formed into arrays. Reviewing the research questions presented at the start of the chapter, the sensors were arrayed into rings each with four sensors protruding outward. Two rings offset by 45 degrees allowed the sensors to monitor the fluid motion around the entire WIDTS. The WIDTS shape was shown to be streamlined and have little to no effect on the sensor output. The best attachment location for the WIDTS was to be carried by the harbor seal in his mouth, allowing the whisker-like sensors to experience the same flow observed by the animal's vibrissae. It was shown the animal was still able to track a hydrodynamic signal with the WIDTS location. Preliminary results show the WIDTS can detect the chase vehicles wake and determine the direction or side of the WIDTS experiencing the wake. 


\section{Chapter 8}

\section{Conclusions and Future Work}

The goal of this research was to combine work from several fields to aid in the development of a biologically inspired whisker-like fluid motion sensor for wake detection and tracking. To accomplish this goal, first, two biological sensory systems were examined to explore their kinematics and material properties. The trichobothria of spider are microscale hairs capable of detecting small air movements. These hair sensors inform the stationary arachnid about its surroundings and provides information about the location of possible predators and prey. Force testing on trichobothria revealed the hair remains rigid under the loads and is supported by a membrane that provides the restoring and damping parameters of the kinematic system. Modeling the motion as a three parameter viscoelastic solid was in good agreement with the experimental results for all loading conditions of the hairs. For the larger angular displacement velocities the hair can be modeled as a simple spring system greatly reducing the model complexity. The second biological system investigated was the vibrissae of pinnipeds. Prior research had shown harbor seals with undulated vibrissae are capable of tracking an underwater object's wake using only these whisker sensors. The vibrissae are controlled by the animal and rigidly held pointing outward when monitoring hydrodynamic stimuli. Unlike the trichobothria, the whiskers bend and deflect under loads and these motions are detected by nerves at the whisker 
base. Tests conducted on whiskers of elephant seals, harbor seals, and California sea lions showed that all three were underdamped with natural frequencies under $100 \mathrm{~Hz}$. Force testing to examine the vibrissae material properties revealed the harbor seal whiskers to be the least stiff. Comparing the whisker's responses to flows, all three species demonstrated matching trends indicating at lower flow speeds the diameter perpendicular to the oncoming flow plays a larger role in the vibrissae motion than the undulatory morphology.

Next, a haircell sensor was modeled to determine the impact of design parameters on sensor response and performance capabilities for wake tracking. Comparing the influence of cantilever thickness and post size showed the cantilever beam has the largest impact on the sensor's deflection under load. However the sensor was limited to accurately capture fluid forces in a single direction and proved fragile. For wake tracking, placing the haircell sensor on the surface of a moving body would likely generate unwanted noise caused by the boundary layer flow blocking important flow information.

Addressing the previous concerns of the haircell sensor, a whisker-like fluid motion sensor was developed based on the spider trichobothria's membrane sensor and the sizing criteria of vibrissae. The sensor's post fit into a gold plated base cone surrounded by a slightly larger cone with four gold plated quadrants. Fluid forces on the post generated rotations causing the distances between the plates on the two cones to change, resulting in a change in capacitance between the four sets of plates. Exciting the sensor with a sinusoidal signal and measuring the change in output voltage across a load resistor provided measurements of the sensor deflection in two orthogonal directions. Similar to the trichobothria the restoring parameters were provided by a PDMS membrane. The design proved usable under a variety of fluids and can be optimized to maximize sensitivity to the fluid environment through modeling of the circuit equation of gain. The design proved to be robust and was able to monitor fluid speeds and directions and well as capture the frequency shedding of cylinder wakes. 
Lastly, the sensor was packaged into arrays and a Wake Information Detection and Tracking System, WIDTS, was developed for deployment on a trained harbor seal, Sprouts, tracking a remote controlled submersible. The WIDTS contained two ring arrays each with four whisker-like sensors. The system was modular and ergonomically designed to be carried in Sprouts mouth just in front of the seal's vibrissae capturing the submarine's wake. The WIDTS protected all necessary electronics to provide the power, excitation signal, data collection, orientation sensors, and bluetooth transmission module. Testing and numerical modeling showed the WIDTS had a slight effect reducing the amplitude of wake signals reaching the seal, but the frequency information remained intact and well above the seal's threshold for capturing fluid motion. Preliminary field trials with Sprouts tracking the submarine while carrying the WIDTS showed the sensors were capable of detecting the sub's wake and indicate the side of the WIDTS from which the signal originated.

This research covering the multi-disciplinary integration of work from biology, material science, electronics, and engineering marks only the beginning of potential in the field of bioinspired sensors. Future work will focus on expanding the knowledge base of what specific flow characteristics influence the animal's decisions in trackingbased performance. Work with Sprouts has shown he is capable of detecting changes in a chase object's speed and when first encountering a wake, he can turn in the direction of the object with high accuracy all while only receiving information through the vibrissae. This leaves open the questions of how exactly does he track? The fluid environment the harbor seal works in is far from ideal. There are numerous noise sources and residual fluid motions from the animal's personal wake. In an attempt to trick the harbor seal a wake from a second object crossed the chase wake. Yet despite this additional information the animal is able to track only the chase object.

Future effort will also focus on the sensor development. There are many features to explore in the sensor improvement. Further work to improve the sensor could examine the membrane characteristics, effect of modifying the post to undulated surface form 
for high speed flows, and improvements to the electronics to reduce noise and improve gain. Further work is also necessary in the signal processing of the sensor output. The idea is to eventually install the whisker-like sensors onto an AUV and track a second submersible using only information obtained through the sensors to track the wake.

Ultimately, the research presented in this dissertation has made contributions to the biomimetic sensor field. Gains are made in the experimentally validated modeling of trichobothria and pinniped vibrissae. The whisker-like sensor proposed and developed here shows potential in tracking hydrodynamic signals. The field remains relatively new and the design space is immense. The biological sensory systems examined here are only a tiny sampling of the whisker and hair sensors found in nature. As conducted here, combining the biologically relevant solutions to fluid motion sensing with engineering methods will offer innovative new solutions to sensor design. 


\section{Bibliography}

[1] Brittinger, W. Trichobothrien, medienstromungen und das orientierungsverhalten von jagdspinnen (Cupiennius salei). Master's thesis, University of Vienna, 1998.

[2] Dehnhardt, G., Mauck, B., Hanke, W., and Bleckmann, H. Hydrodynamic trail-following in harbor seals(Phoca vitulina). Science 293 (2001), 102-104.

[3] Barbier, C., And Humphrey, J. Numerical calculation of the flow in the lateral line canal: applications to predators tracking prey. ASME International Mechanical Engineering Congress and Exposition (2006).

[4] McConney, M., Schaber, C., Julian, M., Eberhardt, W., Humphrey, J., Barth, F., And Tsukruk, V. Surface force spectroscopic point load measurements and viscoelastic modelling of the micromechanical properties of air flow sensitive hairs of a spider (Cupiennius salei). $J R$ Soc Interface 6 (2009), 681-694.

[5] Barth, F., And Holler, A. Dynamics of arthropod filiform hairs. v. the response of spider trichobothria to natural stimuli. Phil Trans $R$ Soc B 354 (1999), 183-192.

[6] Murphy, C., Eberhardt, W., Calhoun, B., Mann, K., and Mann, D. Effect of angle on flow-induced vibrations of pinniped vibrissae. Plos One (in submission).

[7] Chen, N., Tucker, C., Engel, J., Yang, Y., Pandya, S., And Liu, C. Design and characterization of artificial haircell sensor for flow sensing with ultrahigh velocity and angular sensitivity. Journal of Microelectromechanical Systems 16 (2007), 999-1015.

[8] Chen, N. Artificial lateral line canal system for underwater disturbance sensing. Master's thesis, University of Illinois at Urbana-Champaign, 2008.

[9] Barbier, C., Humphrey, J., Paulus, J., And M., A. Design, fabrication and testing of a bioinspired hybrid hair-like fluid medium motion sensor array. ASME Conference Proceeding 8 (2007), 1319-1324. 
[10] Stocking, J. B., Eberhardt, W. C., Shakhsheer, Y. A., Paulus, J. R., Appleby, M., And Calhoun, B. H. A capacitance-based whiskerlike artificial sensor for fluid motion sensing. IEEE Sensors (2010), 2224-2229.

[11] Stocking, J. Numerical and experimental investigation of a whisker-like fluid motion sensor. Master's thesis, University of Virginia, 2010.

[12] Staff, N. R. C. Undersea Vehicles and National Needs. National Academy Press, Washington, DC, 1996.

[13] Denney, M. Blip, Ping, and Buzz...Making Sense of Radar and Sonar. The Johns Hopkins University Press, Baltimore, MD, 1953.

[14] Horton, J. Fundamentals of Sonar. United States Naval Institute, Annapolis, MD, 1957.

[15] Vogel, S. Life in Moving Fluids, 2nd ed. Princeton University Press, Princeton, NJ, 1994.

[16] Coombs, S. Smart skins: Information processing by lateral line flow sensors. Auton. Robots 11 (2001), 255-261.

[17] Patton, P., Windsor, S., And Coombs, S. Active wall following by mexican blind cavefish (Astyanax mexicanus). J Comp Physiol A Neuroethol Sens Neural Behav Physiol 11 (Nov 2010), 853-867.

[18] VAnNetten, S., And Khanna, S. Stiffness changes of the cupula associated with the mechanics of hair cells in the fish lateral line. Proc Nat Acad Sci 91 (1994), 1549-1553.

[19] Dangles, O., Magal, C., Pierre, D., Olivier, A., And Casas, J. Variation in morphology and performance of predator-sensing system in wild cricket populations. Journal of Experimental Biology 208 (2005), 461-468.

[20] Shimozawa, T., Kumagai, T., And BabA, Y. Structural scaling and functional design of the cercal wind-receptor hairs of a cricket. J Comp Phys A 183 (1998), 171-186.

[21] Shimozawa, T., And Kanou, M. Varieties of filiform hairs: Range fractionation by sensory afferents and interneurons of a cricket. J Comp Phys A 155 (1984), 485-493.

[22] Kumagai, T., Shimozawa, T., And Baba, Y. The shape of wind-receptor hairs of cricket and cockroach. J Comp Phys A 183 (1998), 187-192.

[23] BARTh, F. How to catch the wind: spider hairs specialized for sensing the movement of air. Naturwissenschaften 87 (2000), 51-58.

[24] Moller, A. Sensory Systems: Anatomy and Physiology. Academic, San Diego, CA, 2003. 
[25] vannetten, S., Dinklo, T., Marcotti, W., and Kros, C. Channel gating forces govern accuracy of mechano-electrical transduction in hair cells. Proc Nat Acad Sci 100 (2003), 15510-15515.

[26] Dehnhardt, G., Mauk, B., and Bleckmann, H. Seal whiskers detect water movements. Nature 394 (1998), 235-236.

[27] hanke, W., And Bleckmann, H. The hydrodynamic trails of Lepomis gibbosus (centrarchidae), Colomesus psittacus (tetraodontidae) and Thysochromis ansorgii (cichlidae) investigated with scanning particle image velocimetry. Journal of Experimental Biology 207 (2004), 1585-1596.

[28] Nauen, J., And Lauder, G. Quantification of the wake of rainbow trout (Oncorhynchus mykiss) using three-dimensional stereoscopic digital particle image velocimetry. Journal of Experimental Biology 205 (2002), 3271-3279.

[29] Hanke, W., Brucker, C., And Bleckmann, H. The ageing of the low frequency water disturbances caused by swimming goldfish and its possible relevance to prey detection. Journal of Experimental Biology 203 (2000), 11931200 .

[30] Muller, U., Van Den Heuvel, B., Stamhuis, E., And Videler, J. Fish foot prints: Morphology and energetics of the wake behind a continuously swimming mullet (Chelon labrosus risso). Journal of Experimental Biology 200 (1997), 2893-2906.

[31] Videler, J., Muller, U., And Stamhuis, E. Aquatic vertebrate locomotion: Wakes from body waves. Journal of Experimental Biology 202 (1999), 3423-3430.

[32] Nauen, J., And Lauder, G. Hydrodynamics of caudal fin locomotion by chub mackerel, Scomber japonicus (scombridae). Journal of Experimental Biology 205 (2002), 1709-1724.

[33] DRucker, E., And Lauder, G. Experimental hydrodynamics of fish locomotion: Functional insights from wake visualization. Integ and Comp Biol 42 (2002), 243-257.

[34] Liao, J., Beal, D., Lauder, G., and Triantafyllou, M. Fish exploiting vortices decrease muscle activity. Science 302 (2003), 1566-1569.

[35] Cimbala, J., Nagib, H., And Roshko, A. Large structure in the far wakes of two-dimensional bluff bodies. J Fluid Mech 190 (1988), 265-298.

[36] Gerrard, J. The wakes of cylindrical bluff bodies at low reynolds number. Philosophical Transactions of the Royal Society of London. Series A 288 (1978), 351-382. 
[37] Ponta, F., And Aref, H. Strouhal-reynolds number relationship for vortex streets. Physical Review Letters 93 (2004).

[38] Roshko, A. On drag and shedding frequency of two-dimensional bluff bodies. Tech. Rep. 3169, NACA, 1954.

[39] Roshko, A. On the development of turbulent wakes from vortex streets. Tech. Rep. 1191, NACA, 1954.

[40] Williamson, C. Vortex dynamics in the cylinder wake. Annual Review of Fluid Mechanics 28 (1996), 477-539.

[41] Ahlborn, B., Seto, M., And Noack, B. On drag, strouhal number and vortex-street structure. Fluid Dynamics Research 30 (2002), 379-399.

[42] Kelly, J., And van Netten, S. Topography and mechanics of the cupula in the fish lateral line. i. variation of copular structure and composition in three dimensions. Journal of Morphology 207 (1991), 23-26.

[43] Kroese, A., And Schellart, N. Velocity and acceleration-sensitive units in the trunk lateral line of the canal. J. Neurophysiol 68 (1992), 2212-2221.

[44] Chagnaud, B., Bleckmann, H., and Hofmann, M. Karman vortex street detection by the lateral line. J. Comp. Physiol. A 193 (2007), 753-763.

[45] Pohlmann, K., Grasso, F., and Breithaupt, T. Tracking wakes: the nocturnal fishy strategy of piscivorous catfish. Proc. Natl Acad. Sci. USA 98 (2001), 41-51.

[46] Pohlmann, K., Atema, J., And Breithaupt, T. The importance of the lateral line in nocturnal predation of piscivorous catfish. $J$ of exp Biol 207 (2004), 2971-2978.

[47] BARTh, F. A spider's world: Senses and behavior. Springer, New York, NY, 2002.

[48] Humphrey, J., And Barth, F. Insect mechanics and control. Elsevier, Amsterdam, The Netherlands, 2008, ch. Medium flow-sensing hairs: biomechanics and models, pp. 1-80.

[49] Barth, F., Humphrey, J., Wastl, U., Halbritter, J., And BritTINGER, W. Dynamics of arthropod filiform hairs iii. flow patterns related to air movement detection in a spider (Cupiennius salei keys.). Phil. Trans. $R$. Soc. Lond B 347 (1995), 397-412.

[50] Harrison, R. Functional Anatomy of Marine Mammals. Academic Press, London, 1977. 
[51] R.J., S., D., K., D.H., L., B.L., S., And C., R. Why pinnipeds don't echolocate: the role of selection in pinniped biosonar. Journal of the Acoustical Society of America 106 (1999), 1142-1148.

[52] H., H. Diving in darkness; whiskers as sense organs of the ringed seal (Phoca hispida saimensis). J Zool (Lond) 218 (1989), 663-678.

[53] Marshall, C., Amin, H., Kovacs, K., and Lydersen, C. Microstructure and innervation of the mystacial vibrissal follicle-sinus complex in bearded seals, Erignathus barbatus (pinnipedia: Phocidae). The Anatomical Record Part A 288A (2006), 13-25.

[54] Thewissen, J., And Nummela, S. Sensory Evolution on the Threshold: Adaptations in Secondarily Aquatic Vertebrates. University of California Press, Berkley, CA, 2008.

[55] H., H., A., P., U., S., AND I.J., H. Aquatic environment and differentiation of vibrissae: Comparison of sinus hair systems of ringed seal, otter and pole cat. Brain Behav Evol 4 (2009), 268-279.

[56] Wieskotten, S., Dehnhardt, G., Mauck, B., Miersch, L., And Hanke, W. The impact of glide phases on the trackability of hydrodynamic trails in harbour seals (Phoca vitulina). Journal of Exp Biol 213 (2010), 37343740 .

[57] G., D., AND A., K. Sensitivity of the mystacial vibrissae of harbour seals (Phoca vitulina) for size differences of actively touched objects. J Exp Biol 198 (1995), 2317-2323.

[58] Glaser, N., Otter, C., Dehnhardt, G., and Hanke, W. Hydrodynamic trail following in a california sea lion (Zalophus californianus). J Comp Physiol A 197 (2010), 141-151.

[59] Wieskotten, S., Dehnhardt, G., Mauck, B., Miersch, L., And HANKE, W. Hydrodynamic determination of the moving direction of an artificial fin by a harbor seal (Phoca vitulina). J Exp Biol 213 (2010), 2194-2200.

[60] Ilg, U., Blthoff, H., And Mallot, A. Dynamic Perception. IOS Press, Tubugen, 2004.

[61] Ginter, C., Fish, F., And Marshall, C. Morphological analysis of the bumpy profile of phocid vibrissae. Marine Mammal Science 26 (2010), 733745 .

[62] Ginter, C., DeWitt, T., Fish, F., and Marshall, C. Fused traditional and geometric morphometrics demonstrate pinniped whisker diversity. Plosone 7 (2012). 
[63] Reynolds, J., And Rommel, S. Biology of Marine Mammals. Smithsonian Institution Press, 1999.

[64] Hyvarinen, H., and Katajisto, H. Functional structure of the vibrissae of the ringed seal (Phoca hispida Schr.). Acta Zool Fenn 171 (1984), 27-30.

[65] Miersch, L., Hanke, W., Wieskotten, S., Hanke, F., Oeffner, J., Leder, A., Brede, M., Witte, M., And Dehnhardt, G. Flow sensing by pinniped whiskers. Philosophical Transactions of the Royal Society B 366 (2011), 3077-3084.

[66] Kastelein, R., Thomas, J., And Nachtigall, P. Sensory Systems of Aquatic Mammals. De Spil, Woerden, The Netherlands, 1995.

[67] Hanke, W., Wieskotten, S., Miersch, L., Witte, M., Brede, M., Leder, A., And Dehnhardt, G. On the wake flow dynamics behind harbor seal vibrissae a fluid mechanical explanation for an extraordinary capability. Nature-Inspired Fluid Mechanics, NNFM 119 (2012), 271-289.

[68] hanke, W., Witte, M., Miersch, L., Brede, M., Oeffner, J., Michael, M., Hanke, F., And Dehnhardt, G. Harbor seal vibrissae morphology suppresses vortex-induced vibrations. Journal of Experimental Biology 213 (2010), 2665-2672.

[69] Dijkstra, M., van BaAr, J., Wiegerink, R., Lammerink, T., de Boer, J., AND KRIJnen, G. Artificial sensory hairs based on the flow sensitive receptor hairs of crickets. J. Micromech. Microeng 15 (2005), 132-138.

[70] Krijnen, G., Dijkstra, M., van Baar, J., Shankar, S., Kuipers, W., De Boer, R., Altpeter, D., Lammerink, T., and Wiegerink, R. Mems based hair flow-sensors as model system for acoustic perception studies. Nanotechnology 17 (2006), 84-89.

[71] Fan, Z., Chen, J., Zou, J., Bullen, D., Liu, C., and Delcomyn, F. Design and fabrication of artificial lateral line flow sensors. J. Micromech. Microeng 12 (2002), 655-661.

[72] Solomon, J., And Hartmann, M. Sensing features with robotic whiskers. Nature 443 (2006).

[73] Schultz, A., Solomon, J., Peshrin, M., and Hartmann, M. Multifunctional whisker arrays for distance detection, terrain mapping, and object feature extraction. IEEE International Conference on Robotics and Automation (2005).

[74] Lungarella, M., Hafner, V., Pfeifer, R., And Yokoi, H. An artificial whisker sensor for robotics. IEEE/RSJ International Conference on Intelligent Robots and Systems 3 (2002). 
[75] Fend, M. Whisker-based texture discrimination on a mobile robot. Proceedings of the 8th European Conference on Artifical Life (ECAL) (2005), 302-311.

[76] Fend, M., Bovet, S., And HAFner, V. An artificial mouse- a robot with whiskers and vision. Proceedings of the 35th International Symposium on Robotics (ISR) (2004).

[77] Fend, M., Bovet, S., Yokoi, H., And Pfeifer, R. An active artificial whisker array for texture discrimination. IEEE/RSJ International Conference on Inteligent Robots and Systems (IROS) 2 (2003), 1044-1049.

[78] HiLL, J. Investigation of a bio-inspired fluid motion sensor based on seal whiskers. Master's thesis, University of Virginia, 2007.

[79] Barth, F., Wastl, U., Humphrey, J., and Devarakonda, R. Dynamics of arthropod filiform hairs. ii. mechanical properties of spider trichobothria (Cupiennius salei keys.). Phil Trans R Soc B 340 (1993), 445-461.

[80] Christensen, R. Theory of Viscoelasticity, 2nd ed. Dover Publications Inc, Mineola, NY, 2003.

[81] Flugge, W. Viscoelasticity. Blaisdell Publishing Company, Waltham, MA, 1967.

[82] Fung, Y. Biomechanics, 2nd ed. Springer, New York, NY, 1993.

[83] Fuchigami, N., Hazel, J., Gorbunov, V., Stone, M., Grace, M., AND Tsukruk, V. Biological thermal detection. i: ultra-microstructure of pit organs in infra-red imaging snakes. Biomacromolecules 2 (2001), 757-764.

[84] Cohen, J., Cohen, P., West, C., And Aiken, L. Applied Multiple Regression/Correlation Analysis for the Behavioral Sciences. Lawrence Erlbaum Associates, Hillsdale, NJ, 2003.

[85] Humphrey, J., Devarakonda, R., Iglesias, I., and Barth, F. Dynamics of arthropod filiform hairs. i. mathematical modelling of the hair and air motions. Philosophical Transactions: Biological Sciences 340, 1294 (1993).

[86] Humphrey, J., Deverakonda, R., Iglesias, I., and Barth, F. Errata re. humphrey et al 1993. Phil Trans $R$ Soc B 352 (1995).

[87] Denhardt, G., And Kaminski, A. Sensitivity of the mystacial vibrissae of harbor seals (Phoca vitulina) for size differences of actively touched objects. Journal of Experimental Biology 198 (1995), 2317-2323.

[88] Harrison, R. Functional Anatomy of Marine Mammals. Academic Press, New York, NY, 1972.

[89] Mark-10. Series BG User's Guide, July 2010. 
[90] Polytec. Portable Digital Vibrometer PDV-100, September 2008.

[91] Pilkey, W. Formulas for Stress, Strain, and Structural Matrices, 2nd ed. Wiley, Hoboken, NJ, 2005.

[92] Williams, C., And Kramer, E. The advantages of a tapered whisker. Plos One 5 (2010).

[93] Hartmann, M., Johnson, N., Towal, R., And Assad, C. Mechanical characteristics of rat vibrissae: Resonant frequencies amd damping in isolated whiskers and in the awake behaving animal. Journal of Neuroscience 23, 16 (2003), 6510-6519.

[94] Carl, K., Hild, W., Mampel, J., Schilling, C., Uhlig, R., And WitTe, H. Characterization of statical properties of rat's whisker system. IEEE Sesnors 12, 2 (2012), 340-349.

[95] RAo, S. Mechanical Vibrations, 4th ed. Pearson Prentice Hall, Upper Saddle River, NJ, 2004.

[96] Timoshenko, S., And Goodier, J. Theory of Elasticity, 3rd ed. McGraw Hill, New York, NY, 1970.

[97] Graff, K. Wave Motion in Elastic Solids. Dover Publications, Mineola, NY, 1991.

[98] Kundu, P., And Cohen, I. Fluid Mechanics, 4th ed. Academic Press, Burlington, MA, 2008.

[99] White, F. Viscous Fluid Flow, 3rd ed. McGraw Hill, Boston, MA, 2006.

[100] Brennen, C. A review of added mass and fluid inertial forces. Tech. rep., Naval Civil Engineering Laboratory, Port Hueneme, CA, January 1982.

[101] CHO, C.-H. Characterization of young's modulus of silicon versus temperature using a beam defelction method with four-point bending fixture. Current Applied Physics 9 (2009), 538-545.

[102] Roch, I. fabrication and characterization of an su-8 gripper actuated by a shape memory alloy thin film. Institute of Physics 13, 2 (2003), 330.

[103] Munson, B., Young, D., And Okisshi, T. Fundamentals of Fluid Mechanics, 4th ed. John Wiley and Sons Inc, Hoboken, NJ, 2002.

[104] Haberman, R. Applied Partial Differential Equations with Fourier Series and Boundary Value Problems, 4th ed. Pearson Prentice Hall, Upper Saddle River, NJ, 2004.

[105] Schlichting, H. Boundary Layer Theory. McGraw Hill, Boston, MA, 1979. 
[106] Tang, V., Falabella, S., Guethlein, G., Meyer, G., and Sampayan, S. Enhanced resistivity and breakdown strength via a granular two-phase silicone oil and polypropylene mixed media dielectric. IEEE Transactions on Dielectrics and Electrical Insulation 17 (2010), 1221-1224.

[107] Zin, I., LyOn, S., AND Hussain, A. Under-film corrosion of epoxy-coated galvanised steel an eis and svet study of the effect of inhibition at defects. Progress in Organic Coatings 52 (2005), 126-135.

[108] Kahhaleh, K., Vaca-Corts, E., Jirsa, J., Wheat, H., And CarRASQUILLO, R. Corrosion performance of epoxy-coated reinforcementmacrocell tests. Tech. rep., Texas Department of Transportation, Texas, November 1998.

[109] Keane, R. D., And Adrian, R. J. Optimization of particle image velocimeters. part i: Double pulsed systems. Meas. Sci. Technol. 1 (1990), 1202-1215.

[110] Keane, R. D., And Adrian, R. J. Theory of cross-correlation analysis of piv images. Applied Scientific Research 49 (1992), 191-215.

[111] TSI InCORPORATED. Particle Image Velocimetry (PIV): Quantitative Flow Visualization, 2007.

[112] Boillot, A., And Prasad, A. Optimization proceedure for pulse separation in cross-correlation piv. Experiments in Fluids 21 (1996), 87-93.

[113] Hart, D. Piv error correction. Experiments in Fluids 29 (2000), 13-22.

[114] Mentor, F. Two-equation eddy-viscosity turbulence models for engineering applications. AIAA 32 (1994), 1598-1605.

[115] Hanjalic, K., Nagano, Y., And Tummers, M. Turbulence, Heat and Mass Transfer 4. Begell House, Redding, CT, 2003.

[116] Menter, F. Review of the shear-stress transport turbulence model experience from an industrial perspective. International Journal of Cimputational Fluid Dynamics 23, 4 (2009), 305-316.

[117] El-Behery, S., And Hamed, M. A comparative study of turbulence models performance for turbulent flow in a planar asymmetric diffuser. World Academy of Science, Engineering and Technology 53 (2009), 769-780. 


\section{Appendix A}

\section{Propagation of Error}

The resulting standard deviation of two parameters combined through addition $(r=x+y)$ is:

$$
\sigma_{r}=\sqrt{\sigma_{x}^{2}+\sigma_{y}^{2}}
$$

The resulting standard deviation of two parameters combined through division $(r=x / y)$ is:

$$
\sigma_{r}=\sqrt{\left(y \sigma_{x}\right)^{2}+\left(x \sigma_{y}\right)^{2}}
$$

Using these relationships, the standard deviations of $\beta$ for the parameter fitting can be resolved to the standard deviations for the individual spring and dash pot parameters. 


\section{Appendix B}

\section{Beam Coding}

\section{B.1 Matlab code for the explicit Runge-Kutta fi- nite difference method}

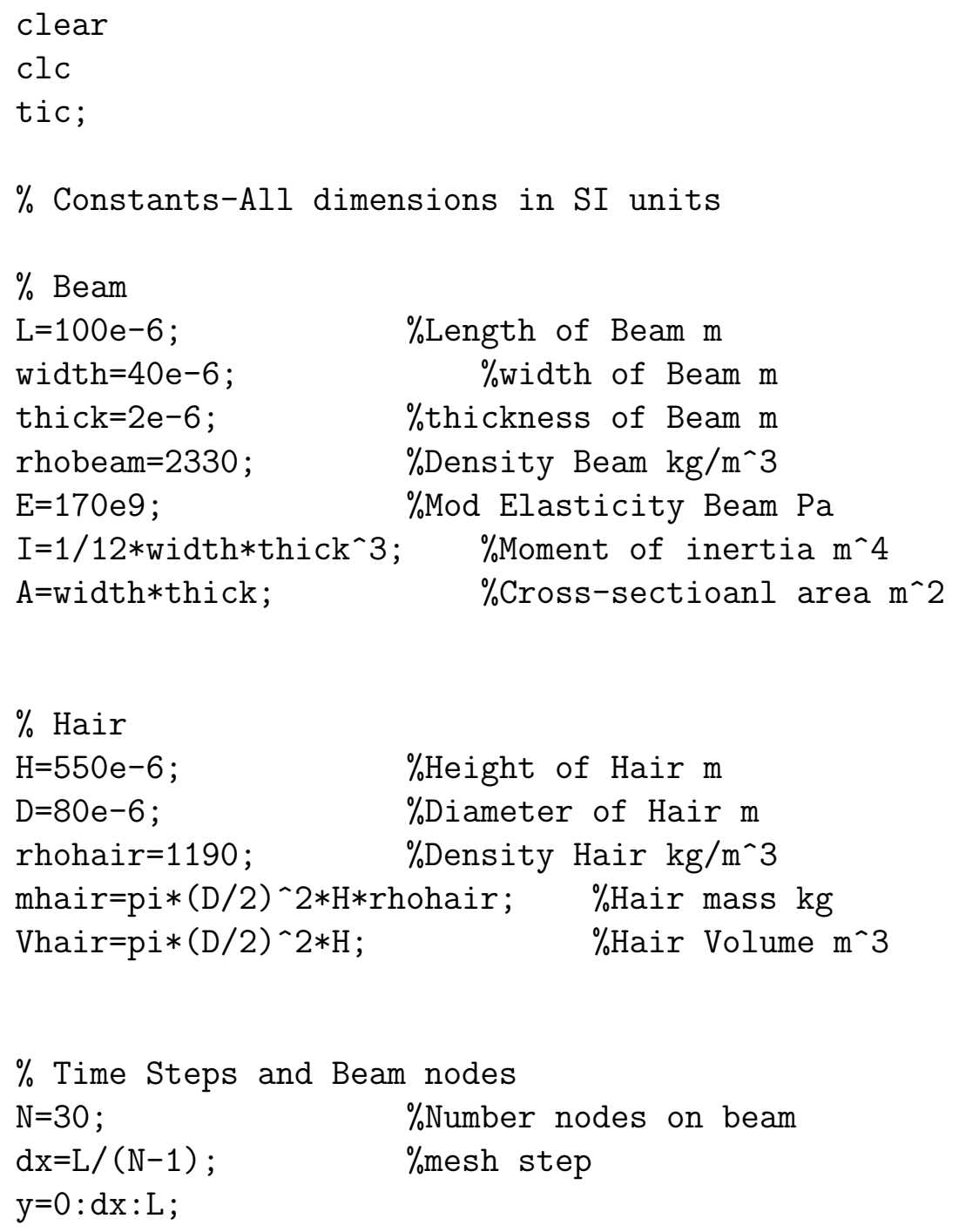




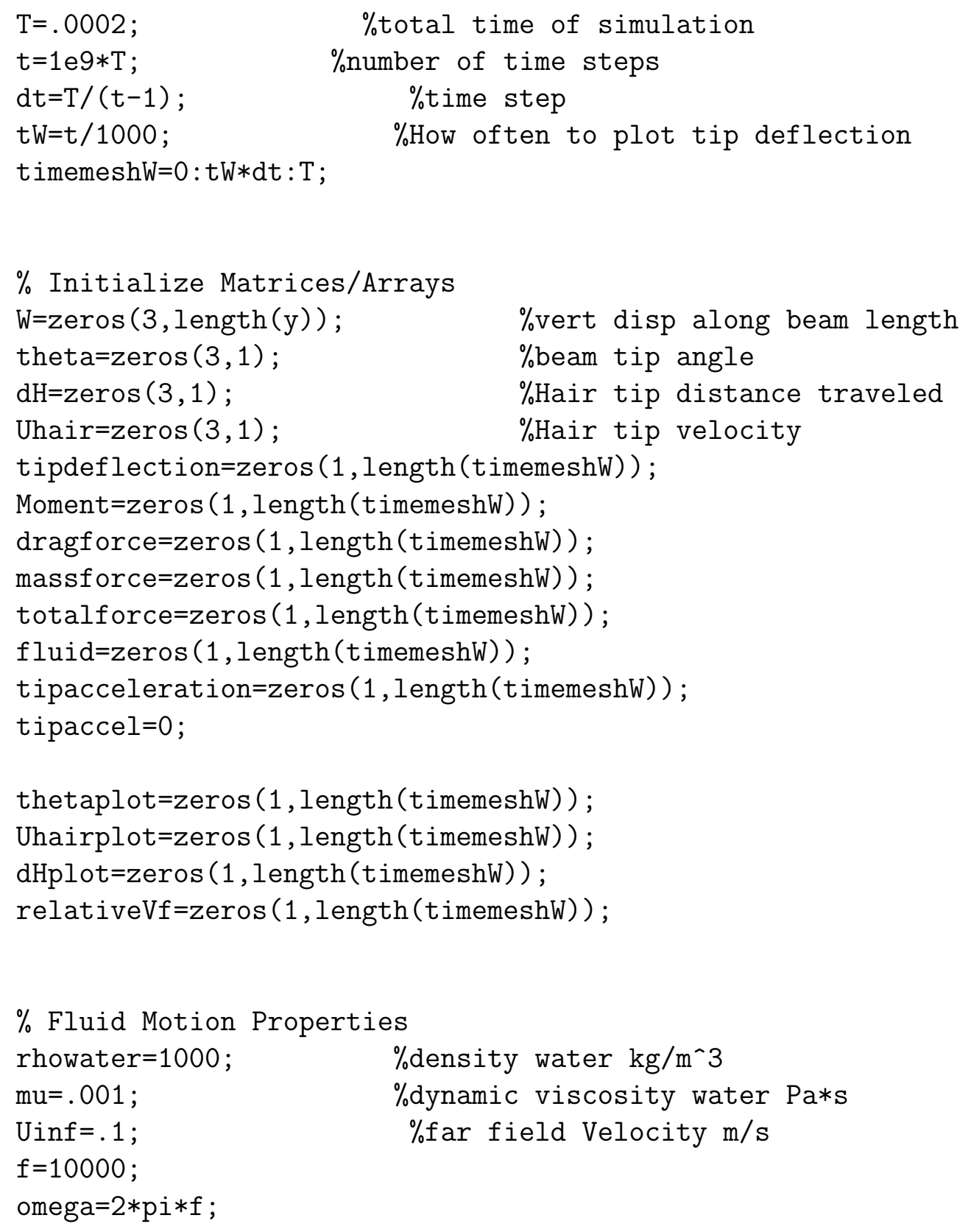




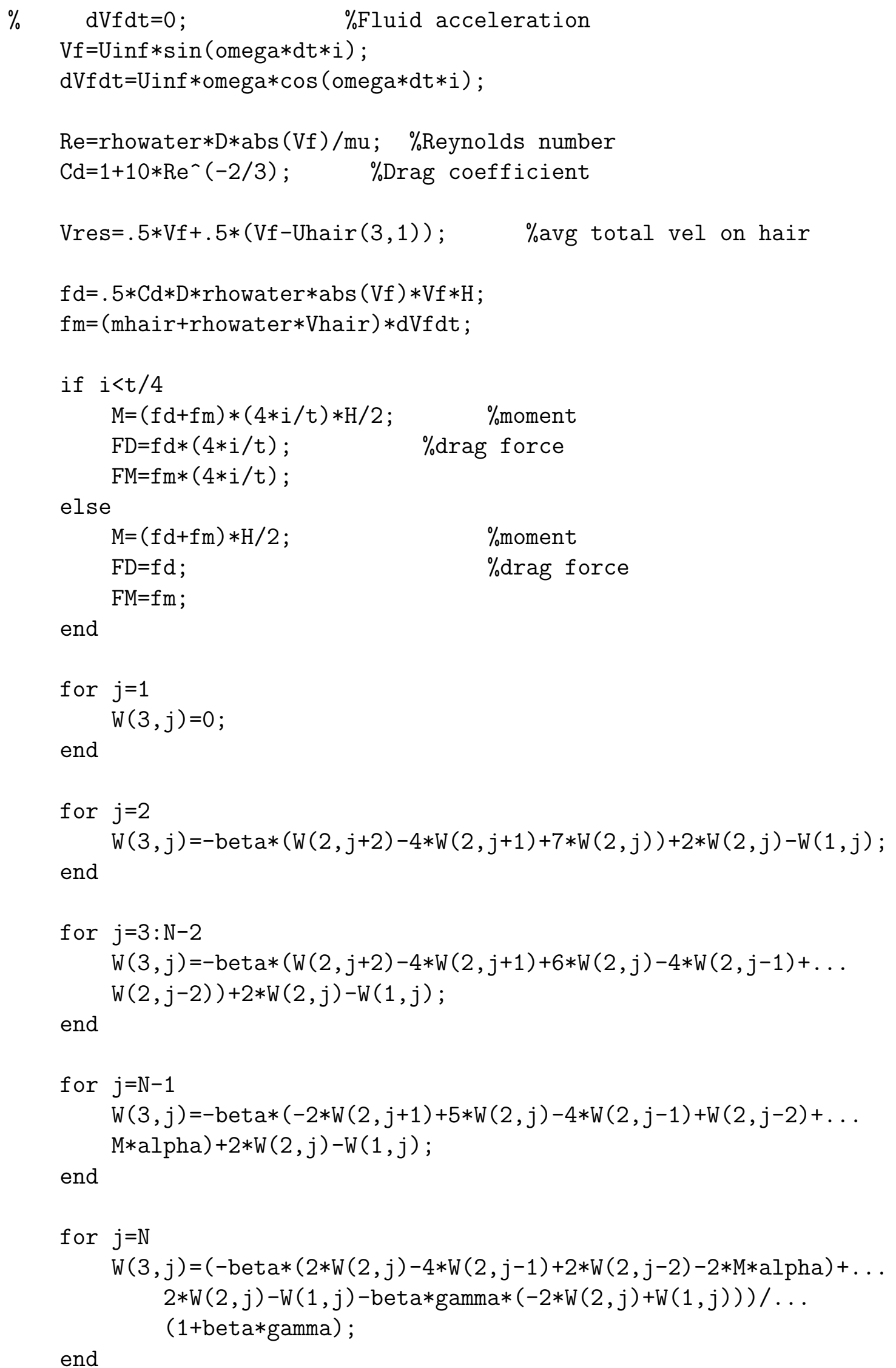




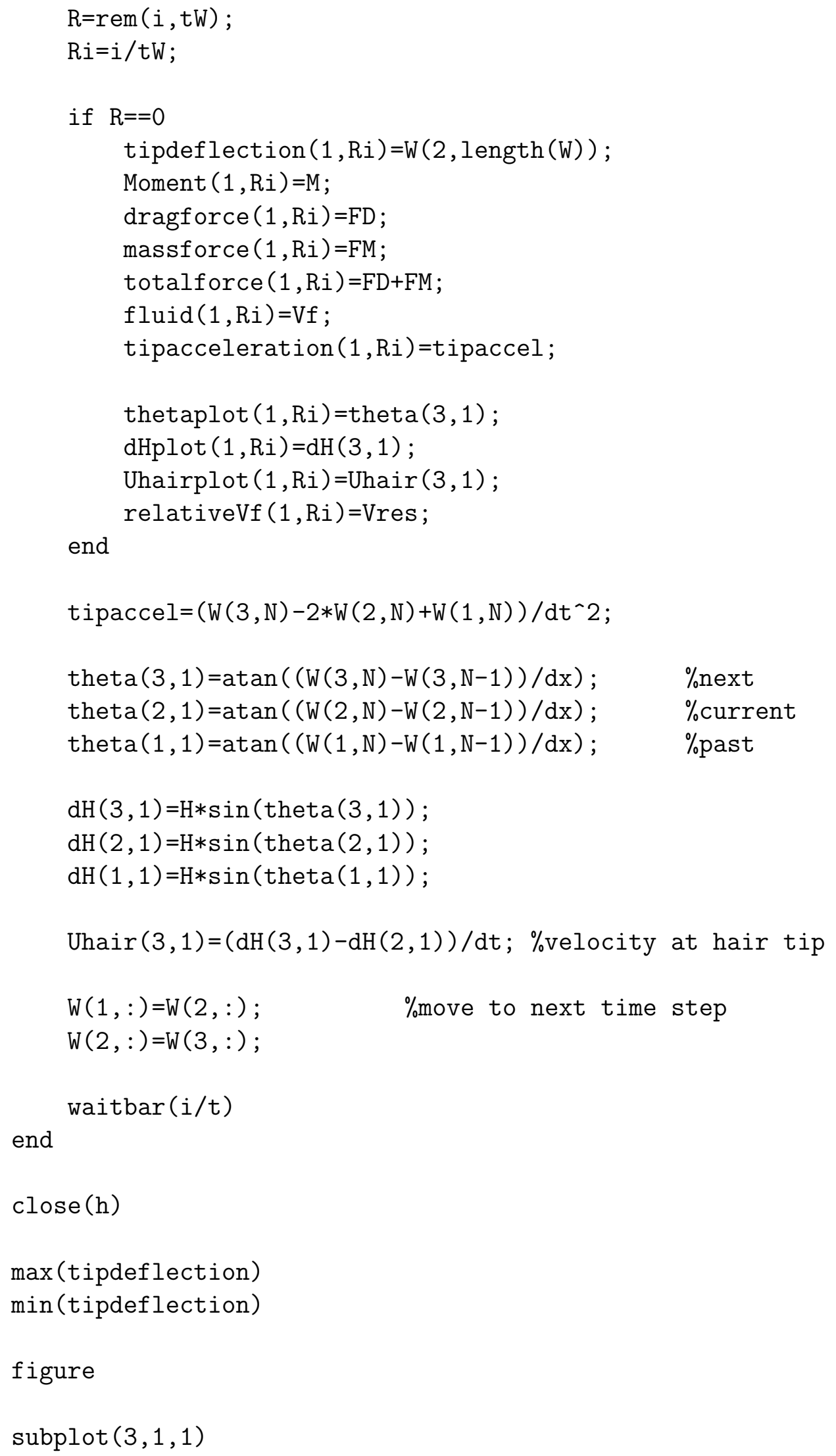




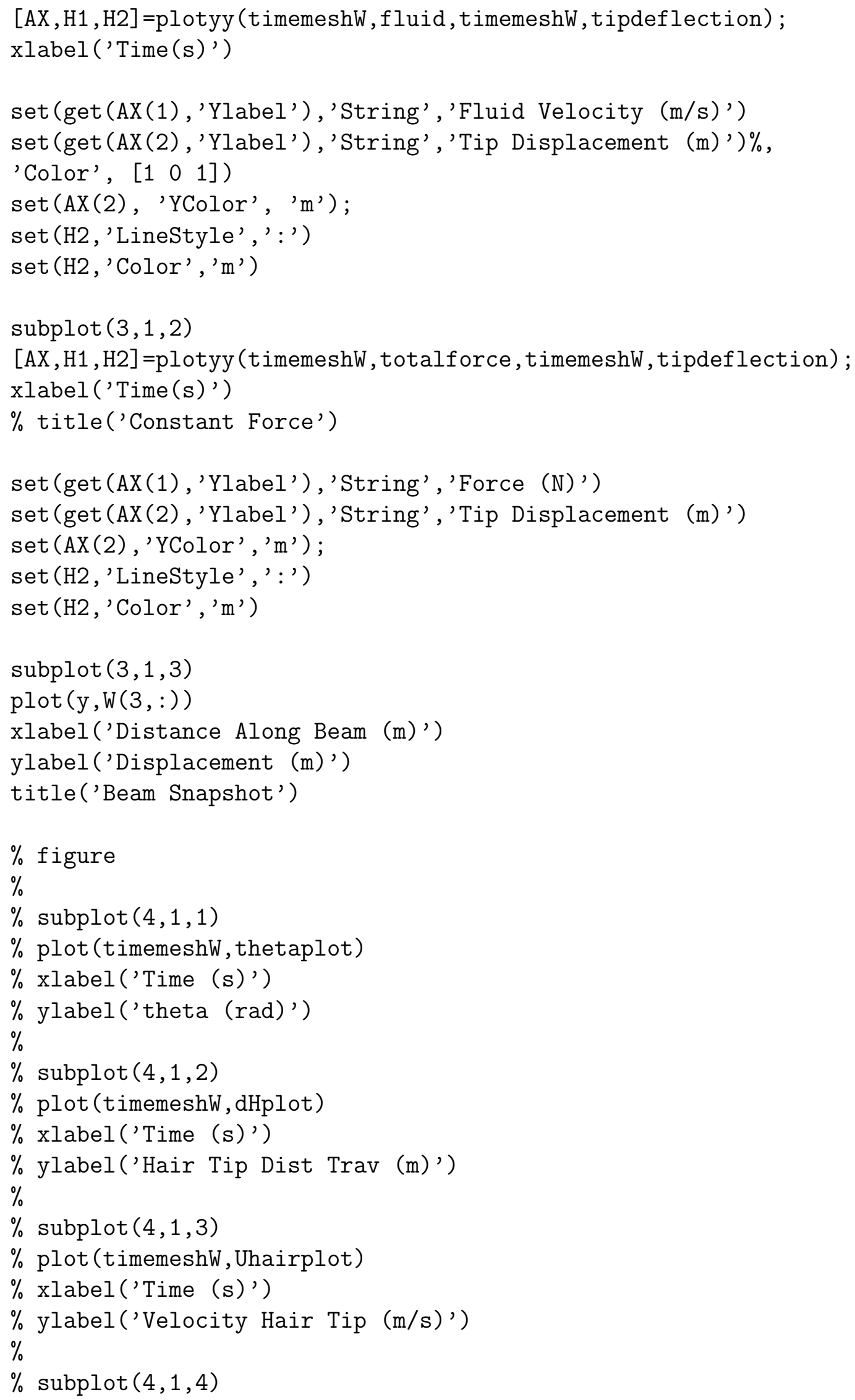




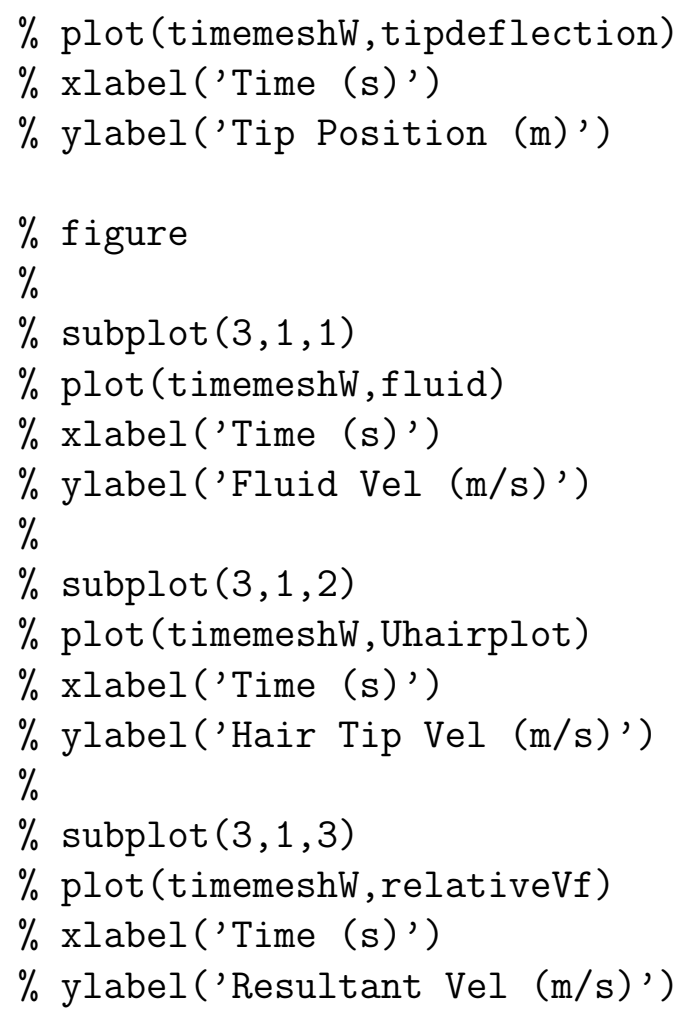




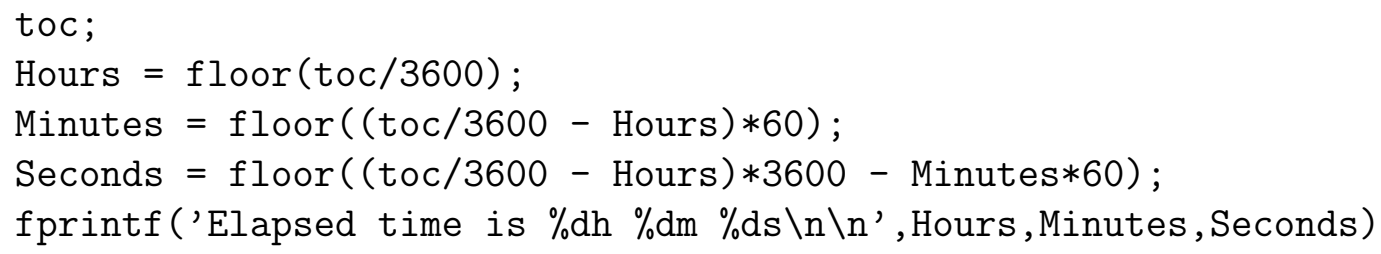

\section{B.2 Matlab code for the implicit Crank-Nicolson finite difference method}

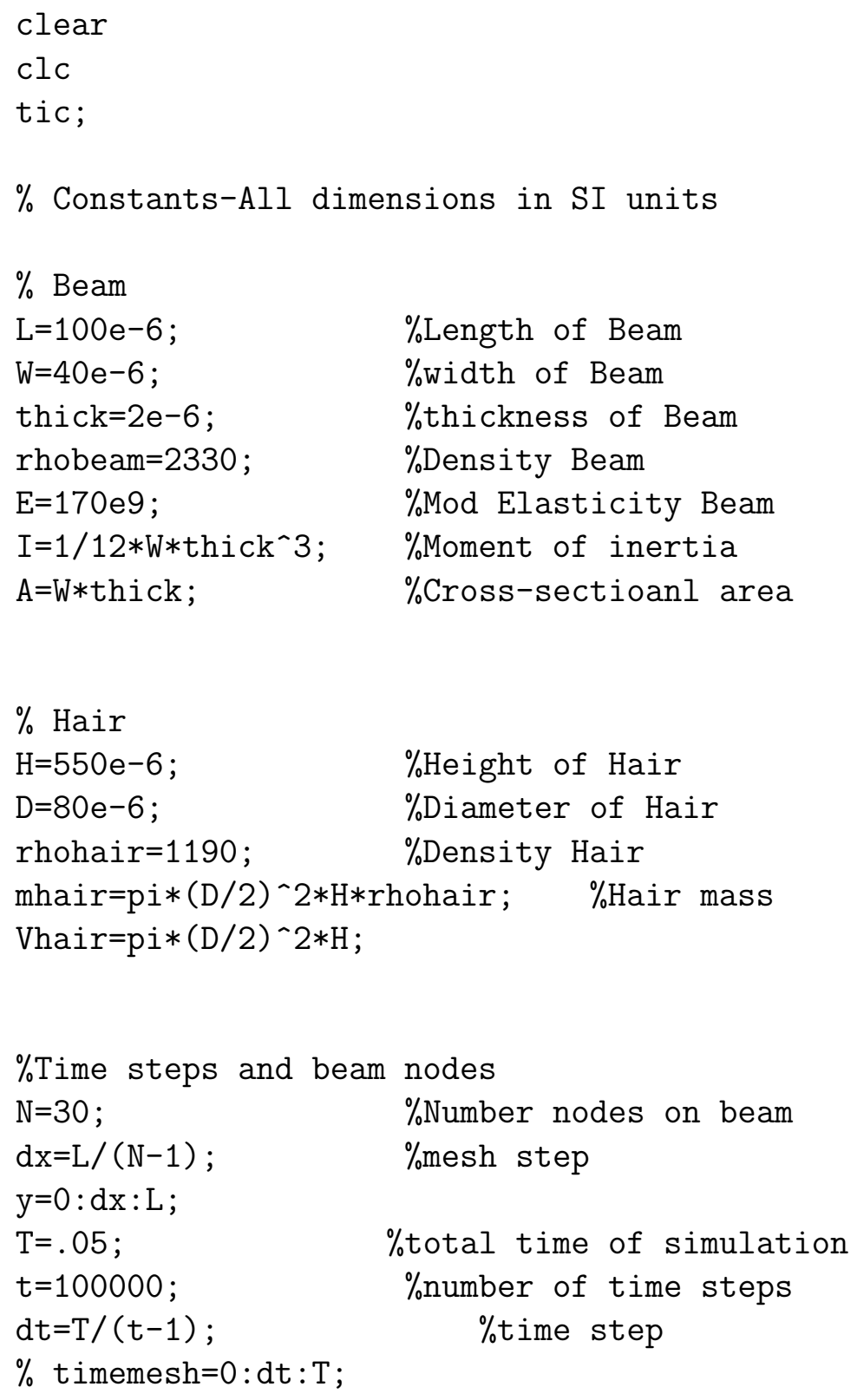




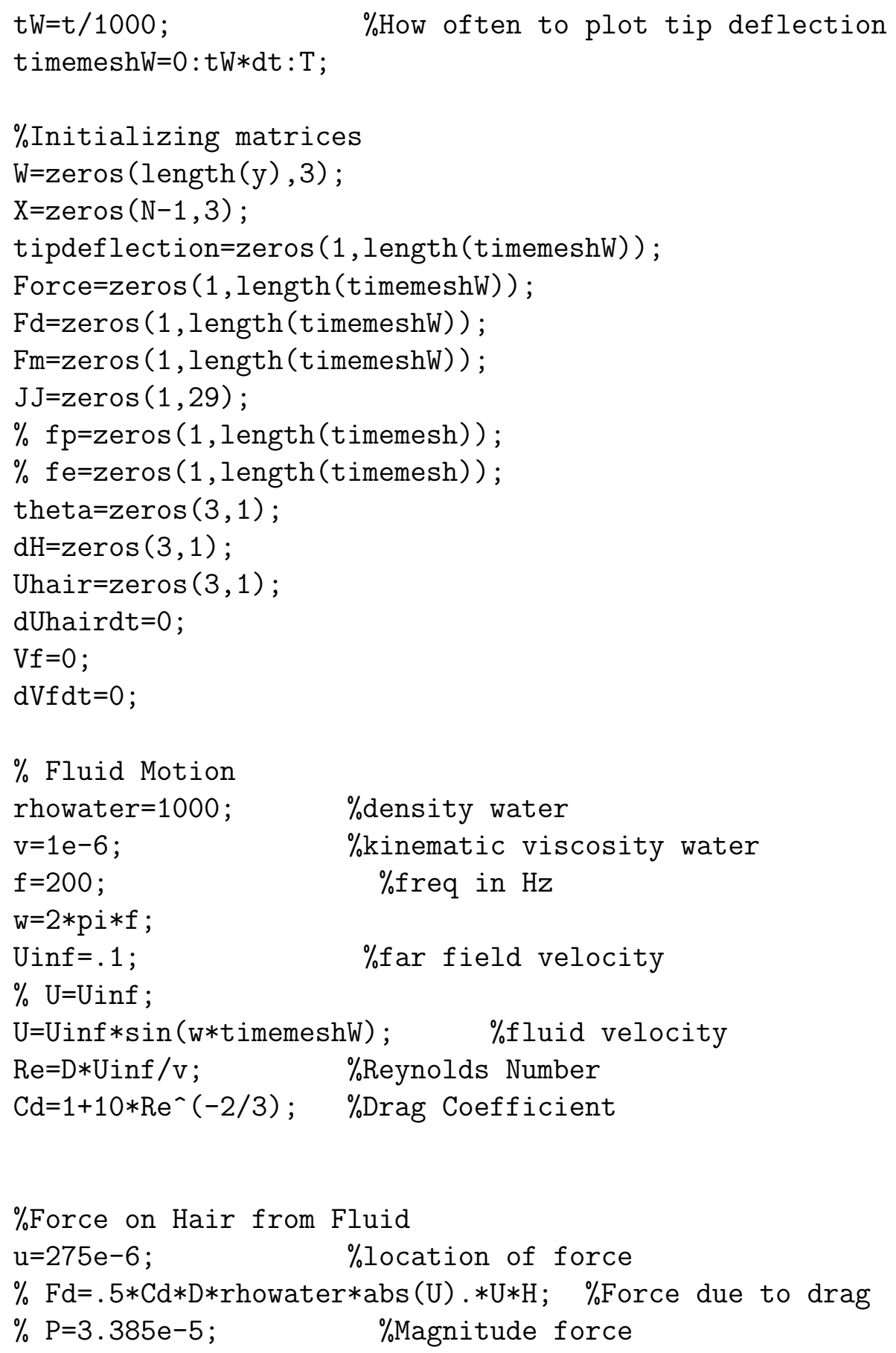


$\mathrm{h}=$ waitbar $(0$, 'Please wait...');

for $i=1: t$

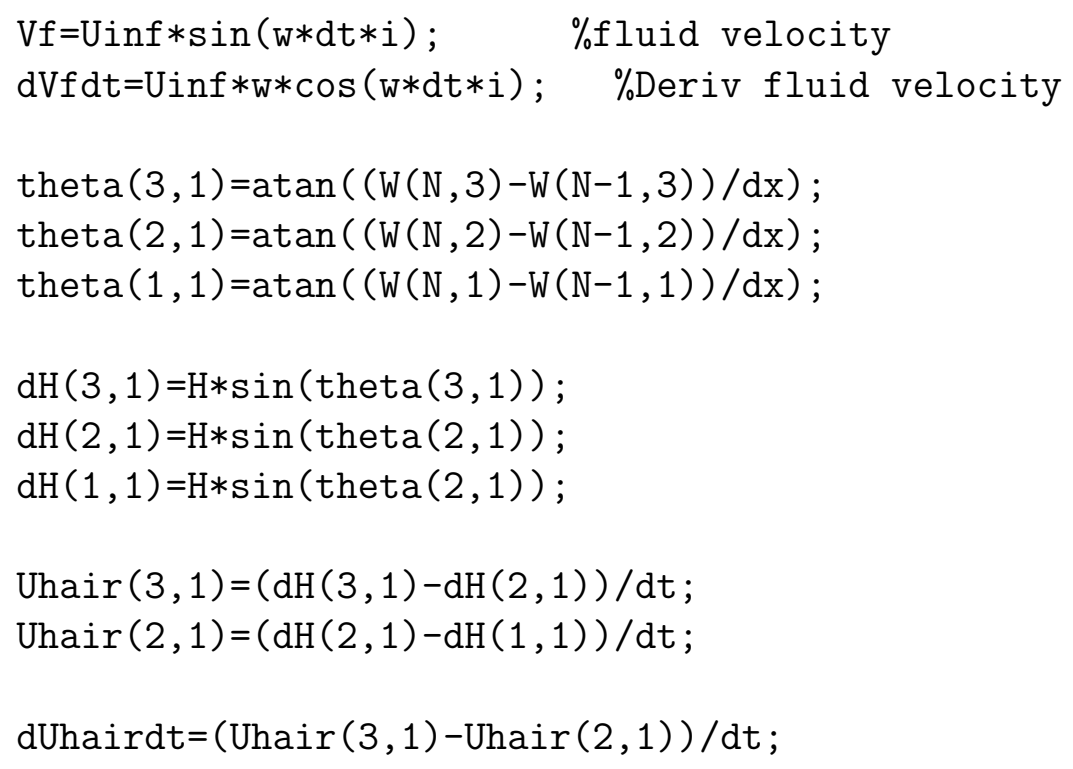

end

$\%$ Constants

$\mathrm{k}=(\mathrm{E} * \mathrm{I}) /\left(\right.$ rhobeam $\left.* \mathrm{~A} * \mathrm{dx}^{\wedge} 4\right)$;

$\mathrm{I}=(\mathrm{Q}) /\left(\right.$ rhobeam $\left.* \mathrm{~A} * \mathrm{dx}^{\wedge} 2\right)$;

$\mathrm{c}=1 / \mathrm{dt}^{\wedge} 2$;

$\mathrm{g}=\mathrm{dx} \wedge 2 * \mathrm{Q} * \mathrm{u} /(\mathrm{E} * \mathrm{I})$;

$\mathrm{s}=\mathrm{mhair} * \mathrm{dx} \wedge 3 /\left(\mathrm{E} * \mathrm{I} * \mathrm{dt}^{\wedge} 2\right)$; 
$\mathrm{YYY}=[7 * \mathrm{k}-2 * 1+\mathrm{c} \quad 1-4 * \mathrm{k} \mathrm{k} \quad 0 \quad 0$ zeros $00000 ; \ldots$ $1-4 * \mathrm{k} 6 * \mathrm{k}-2 * 1+\mathrm{c} 1-4 * \mathrm{k} \mathrm{k} 00$ zeros $0000 ; \ldots$

$\mathrm{k} 1-4 * \mathrm{k} 6 * \mathrm{k}-2 * \mathrm{l}+\mathrm{c} \quad 1-4 * \mathrm{k} \mathrm{k} 00$ zeros $0000 ; \ldots$ $0 \mathrm{k} 1-4 * \mathrm{k} \quad 6 * \mathrm{k}-2 * 1+\mathrm{c} 1-4 * \mathrm{k} \mathrm{k} 00$ zeros $0000 \%$

$00 \mathrm{k} 1-4 * \mathrm{k} 6 * \mathrm{k}-2 * 1+\mathrm{c} 1-4 * \mathrm{k}$ k 00 zeros $0000 ; \ldots$

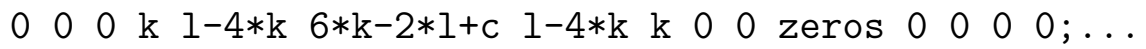
$0000 \mathrm{k} \mathrm{l-4* \textrm {k }} 6 * \mathrm{k}-2 * 1+\mathrm{c} \quad 1-4 * \mathrm{k} \mathrm{k} \quad 0$ zeros $0000 ; \ldots$

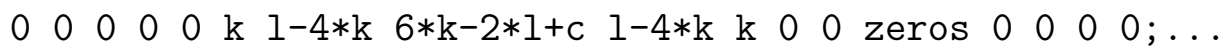

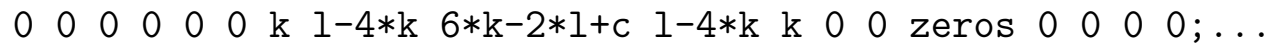

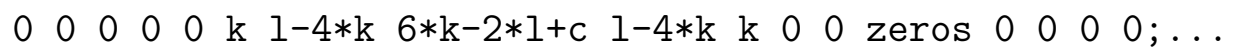

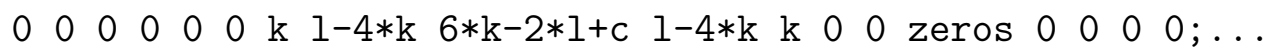

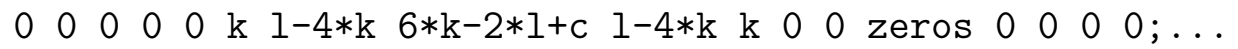
00 zeros $00 \mathrm{k} \mathrm{l} 1-4 * \mathrm{k} 6 * \mathrm{k}-2 * 1+\mathrm{c} 1-4 * \mathrm{k} \mathrm{k} 00$ zeros 000

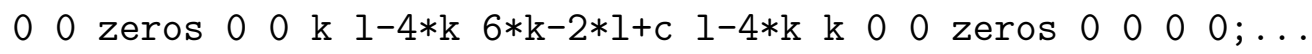
00 zeros $00 \mathrm{k} 1-4 * \mathrm{k} 6 * \mathrm{k}-2 * 1+\mathrm{c} 1-4 * \mathrm{k} \mathrm{k} 00$ zeros $000 ; \ldots$

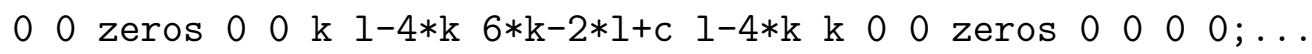
00 zeros $00 \mathrm{k} \mathrm{l} 1-4 * \mathrm{k} \quad 6 * \mathrm{k}-2 * 1+\mathrm{c} \quad 1-4 * \mathrm{k} k \begin{array}{llllll}0 & 0 & \text { zeros } & 0 & 0 & 0 ; \ldots\end{array}$

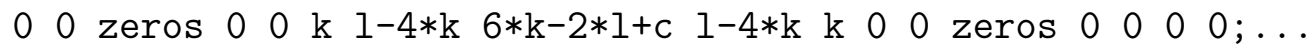

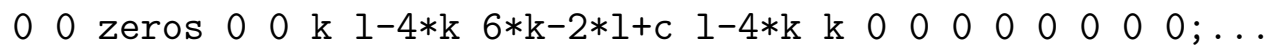

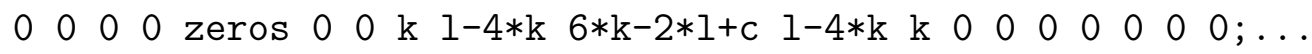

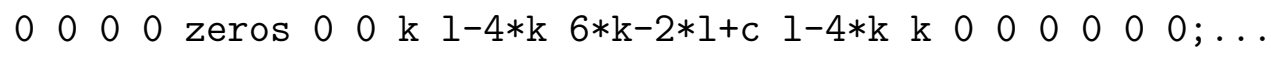

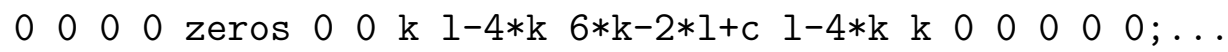
0000 zeros $00 \mathrm{k} 1-4 * \mathrm{k} \quad 6 * \mathrm{k}-2 * 1+\mathrm{c} \quad 1-4 * \mathrm{k} \mathrm{k} \quad 0 \quad 0 \quad 0 \quad 0 ; \ldots$ 0000 zeros $00 \mathrm{k} 1-4 * \mathrm{k} \quad 6 * \mathrm{k}-2 * 1+\mathrm{c} 1-4 * \mathrm{k} \mathrm{k} \quad 0 \quad 0 \quad 0 ; \ldots$ $0 \quad 000$ zeros $00 \mathrm{k} 1-4 * \mathrm{k} \quad 6 * \mathrm{k}-2 * 1+\mathrm{c} 1-4 * \mathrm{k} \mathrm{k} \quad 0 \quad 0 ; \ldots$ 0000 zeros $00 \mathrm{k} \mathrm{l} 1-4 * \mathrm{k} \quad 6 * \mathrm{k}-2 * 1+\mathrm{c} \quad 1-4 * \mathrm{k} \mathrm{k} \quad 0 ; \ldots$ $0 \quad 0 \quad 0 \quad 0$ zeros $0000 \mathrm{k} 1-4 * \mathrm{k} \quad 6 * \mathrm{k}-2 * 1+\mathrm{c} 1-4 * \mathrm{k} \mathrm{k} ; \ldots$ 00000 zeros $0000 \mathrm{k} 1-4 * \mathrm{k} \quad 5 * \mathrm{k}-2 * 1+\mathrm{c} \quad 1-2 * \mathrm{k} ; \ldots$ $\begin{array}{lllllllllll}0 & 0 & 0 & 0 & \text { zeros } & 0 & 0 & 2 * \mathrm{k} & 0 & 2 * \mathrm{k}+\mathrm{k} * \mathrm{~s}+\mathrm{c}] \text {; }\end{array}$

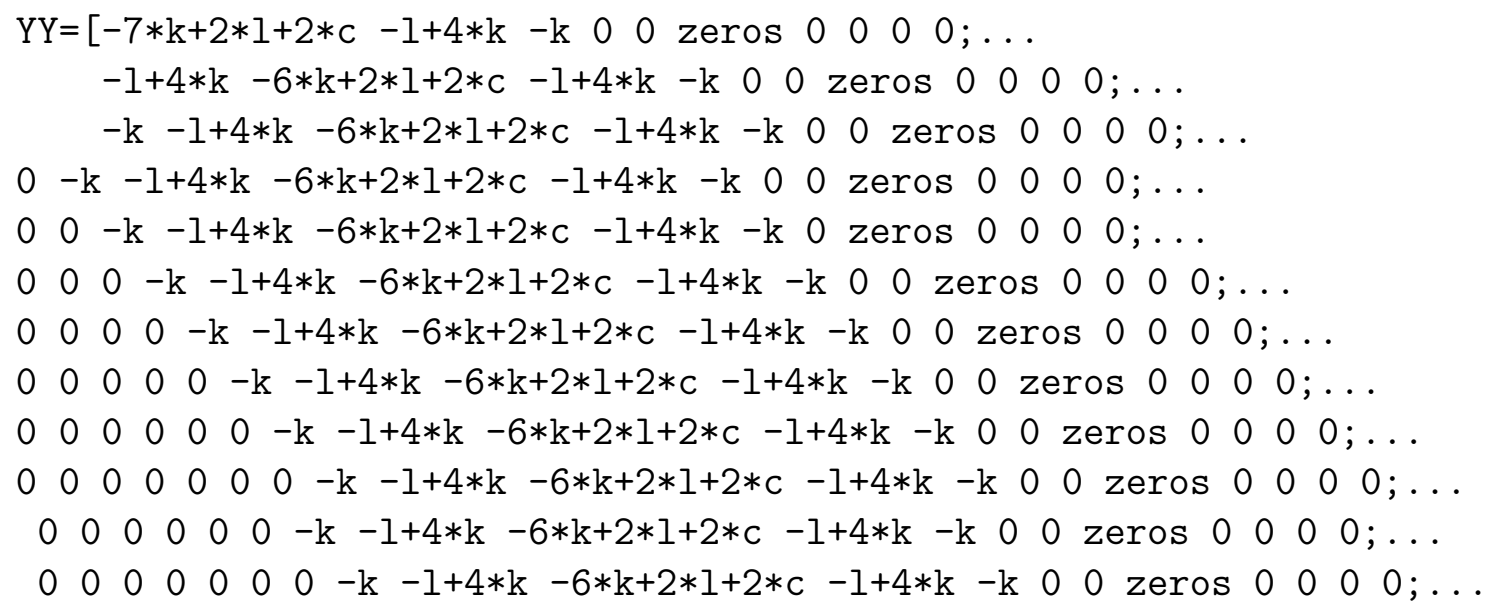




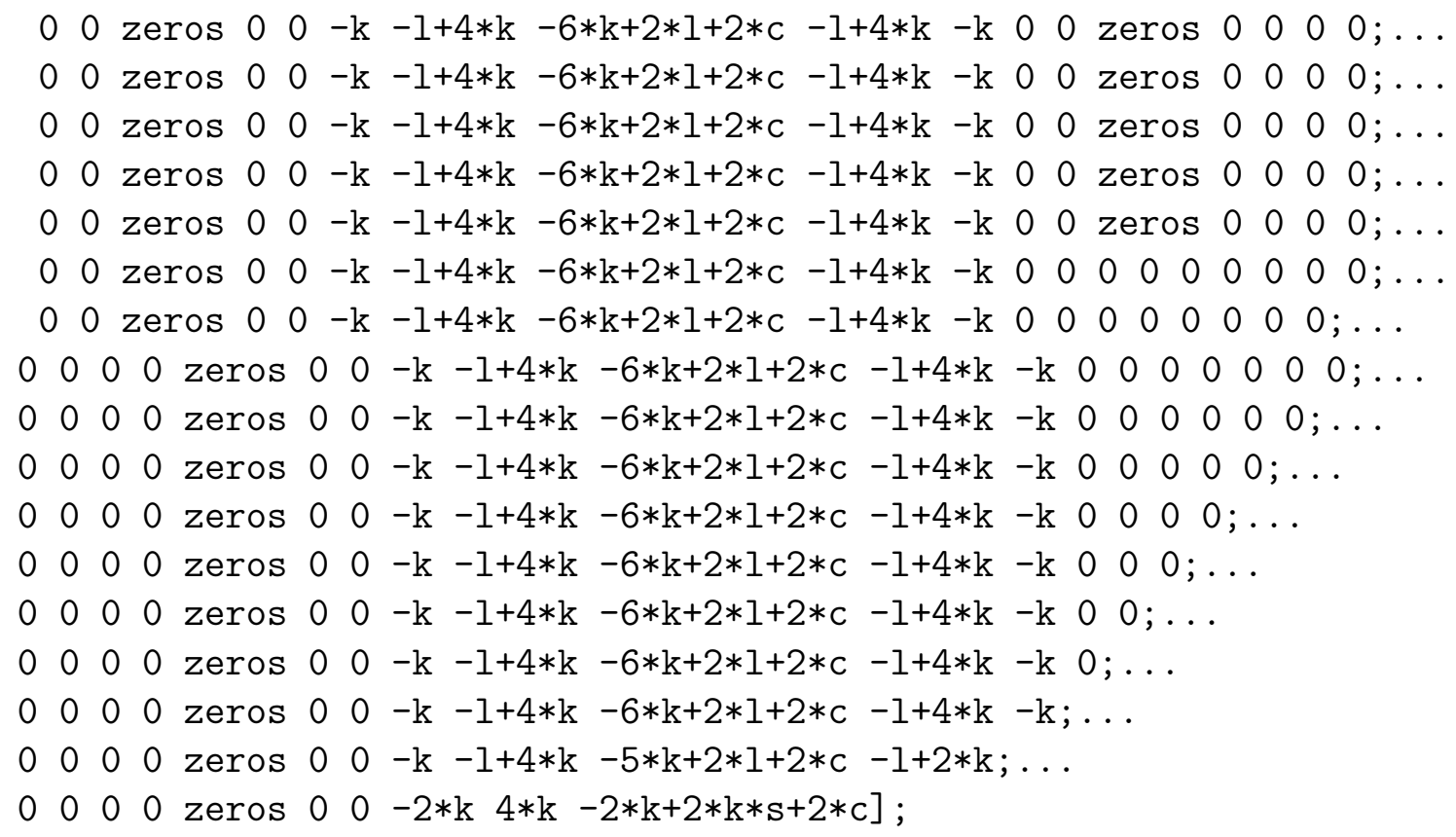

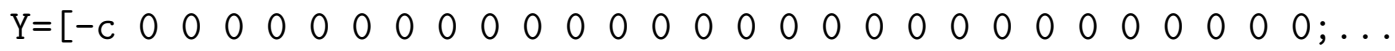

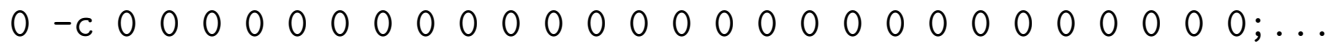

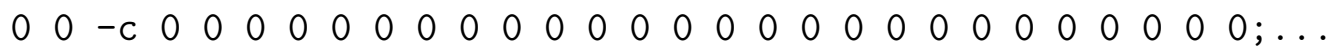

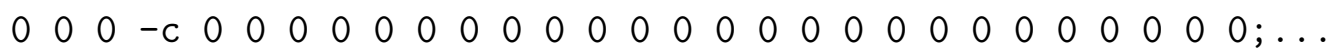
$0 \begin{array}{llllllllllllllllllllllllllll}0 & 0 & 0 & -c & 0 & 0 & 0 & 0 & 0 & 0 & 0 & 0 & 0 & 0 & 0 & 0 & 0 & 0 & 0 & 0 & 0 & 0 & 0 & 0 & 0 & 0 & 0 & 0\end{array} \ldots$ $0 \begin{array}{llllllllllllllllllllllllllll}0 & 0 & 0 & 0 & -c & 0 & 0 & 0 & 0 & 0 & 0 & 0 & 0 & 0 & 0 & 0 & 0 & 0 & 0 & 0 & 0 & 0 & 0 & 0 & 0 & 0 & 0 & 0\end{array} \ldots$ $0 \begin{array}{llllllllllllllllllllllllllll}0 & 0 & 0 & 0 & 0 & -c & 0 & 0 & 0 & 0 & 0 & 0 & 0 & 0 & 0 & 0 & 0 & 0 & 0 & 0 & 0 & 0 & 0 & 0 & 0 & 0 & 0 & 0\end{array} \ldots$ $0 \begin{array}{llllllllllllllllllllllllllll}0 & 0 & 0 & 0 & 0 & 0 & -c & 0 & 0 & 0 & 0 & 0 & 0 & 0 & 0 & 0 & 0 & 0 & 0 & 0 & 0 & 0 & 0 & 0 & 0 & 0 & 0 & 0\end{array} \ldots$ $0 \begin{array}{llllllllllllllllllllllllllll}0 & 0 & 0 & 0 & 0 & 0 & 0 & -c & 0 & 0 & 0 & 0 & 0 & 0 & 0 & 0 & 0 & 0 & 0 & 0 & 0 & 0 & 0 & 0 & 0 & 0 & 0 & 0\end{array} \ldots$ $0 \begin{array}{lllllllllllllllllllllllllllll}0 & 0 & 0 & 0 & 0 & 0 & 0 & 0 & -c & 0 & 0 & 0 & 0 & 0 & 0 & 0 & 0 & 0 & 0 & 0 & 0 & 0 & 0 & 0 & 0 & 0 & 0 & 0 & \ldots\end{array}$

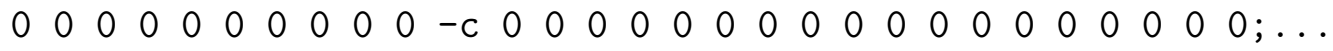
$0 \begin{array}{llllllllllllllllllllllllllll}0 & 0 & 0 & 0 & 0 & 0 & 0 & 0 & 0 & 0 & -c & 0 & 0 & 0 & 0 & 0 & 0 & 0 & 0 & 0 & 0 & 0 & 0 & 0 & 0 & 0 & 0 & 0\end{array} \ldots$ $0 \begin{array}{llllllllllllllllllllllllllll}0 & 0 & 0 & 0 & 0 & 0 & 0 & 0 & 0 & 0 & 0 & -c & 0 & 0 & 0 & 0 & 0 & 0 & 0 & 0 & 0 & 0 & 0 & 0 & 0 & 0 & 0 & 0\end{array} \ldots$ $0 \begin{array}{llllllllllllllllllllllllllll}0 & 0 & 0 & 0 & 0 & 0 & 0 & 0 & 0 & 0 & 0 & 0 & -c & 0 & 0 & 0 & 0 & 0 & 0 & 0 & 0 & 0 & 0 & 0 & 0 & 0 & 0 & 0\end{array} \ldots$

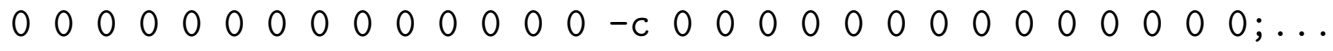
$0 \begin{array}{llllllllllllllllllllllllllll}0 & 0 & 0 & 0 & 0 & 0 & 0 & 0 & 0 & 0 & 0 & 0 & 0 & 0 & -c & 0 & 0 & 0 & 0 & 0 & 0 & 0 & 0 & 0 & 0 & 0 & 0 & 0 ; \ldots\end{array}$ $0 \begin{array}{llllllllllllllllllllllllllll}0 & 0 & 0 & 0 & 0 & 0 & 0 & 0 & 0 & 0 & 0 & 0 & 0 & 0 & 0 & -c & 0 & 0 & 0 & 0 & 0 & 0 & 0 & 0 & 0 & 0 & 0 & 0\end{array} \ldots$ $0 \begin{array}{llllllllllllllllllllllllllll}0 & 0 & 0 & 0 & 0 & 0 & 0 & 0 & 0 & 0 & 0 & 0 & 0 & 0 & 0 & 0 & -c & 0 & 0 & 0 & 0 & 0 & 0 & 0 & 0 & 0 & 0 & 0\end{array} \ldots$ $0 \begin{array}{llllllllllllllllllllllllllll}0 & 0 & 0 & 0 & 0 & 0 & 0 & 0 & 0 & 0 & 0 & 0 & 0 & 0 & 0 & 0 & 0 & -c & 0 & 0 & 0 & 0 & 0 & 0 & 0 & 0 & 0 & 0\end{array} \ldots$ $0 \begin{array}{llllllllllllllllllllllllllll}0 & 0 & 0 & 0 & 0 & 0 & 0 & 0 & 0 & 0 & 0 & 0 & 0 & 0 & 0 & 0 & 0 & -c & 0 & 0 & 0 & 0 & 0 & 0 & 0 & 0 & 0 ; \ldots\end{array}$ $0 \begin{array}{llllllllllllllllllllllllllll}0 & 0 & 0 & 0 & 0 & 0 & 0 & 0 & 0 & 0 & 0 & 0 & 0 & 0 & 0 & 0 & 0 & 0 & 0 & -c & 0 & 0 & 0 & 0 & 0 & 0 & 0 & 0\end{array} \ldots$ $0 \begin{array}{llllllllllllllllllllllllllll}0 & 0 & 0 & 0 & 0 & 0 & 0 & 0 & 0 & 0 & 0 & 0 & 0 & 0 & 0 & 0 & 0 & 0 & 0 & -c & 0 & 0 & 0 & 0 & 0 & 0 & 0 ; \ldots\end{array}$ $0 \begin{array}{llllllllllllllllllllllllllll}0 & 0 & 0 & 0 & 0 & 0 & 0 & 0 & 0 & 0 & 0 & 0 & 0 & 0 & 0 & 0 & 0 & 0 & 0 & 0 & 0 & -c & 0 & 0 & 0 & 0 & 0 & 0\end{array} \ldots$ $0 \begin{array}{llllllllllllllllllllllllllll}0 & 0 & 0 & 0 & 0 & 0 & 0 & 0 & 0 & 0 & 0 & 0 & 0 & 0 & 0 & 0 & 0 & 0 & 0 & 0 & 0 & 0 & -c & 0 & 0 & 0 & 0 & 0\end{array} \ldots$ $0 \begin{array}{llllllllllllllllllllllllllll}0 & 0 & 0 & 0 & 0 & 0 & 0 & 0 & 0 & 0 & 0 & 0 & 0 & 0 & 0 & 0 & 0 & 0 & 0 & 0 & 0 & 0 & -c & 0 & 0 & 0 & 0 & \ldots\end{array} \ldots$ $0 \begin{array}{llllllllllllllllllllllllllll}0 & 0 & 0 & 0 & 0 & 0 & 0 & 0 & 0 & 0 & 0 & 0 & 0 & 0 & 0 & 0 & 0 & 0 & 0 & 0 & 0 & 0 & 0 & -c & 0 & 0 & 0 ; \ldots\end{array}$ 
$\begin{array}{llllllllllllllllllllllllllllll}0 & 0 & 0 & 0 & 0 & 0 & 0 & 0 & 0 & 0 & 0 & 0 & 0 & 0 & 0 & 0 & 0 & 0 & 0 & 0 & 0 & 0 & 0 & 0 & 0 & 0 & -c & 0 & 0 & \ldots\end{array}$ $0 \begin{array}{lllllllllllllllllllllllllllll}0 & 0 & 0 & 0 & 0 & 0 & 0 & 0 & 0 & 0 & 0 & 0 & 0 & 0 & 0 & 0 & 0 & 0 & 0 & 0 & 0 & 0 & 0 & 0 & 0 & 0 & -c & 0 & \ldots\end{array}$ $0 \begin{array}{llllllllllllllllllllllllllll}0 & 0 & 0 & 0 & 0 & 0 & 0 & 0 & 0 & 0 & 0 & 0 & 0 & 0 & 0 & 0 & 0 & 0 & 0 & 0 & 0 & 0 & 0 & 0 & 0 & 0 & 0 & -c-k * s] \text {; }\end{array}$

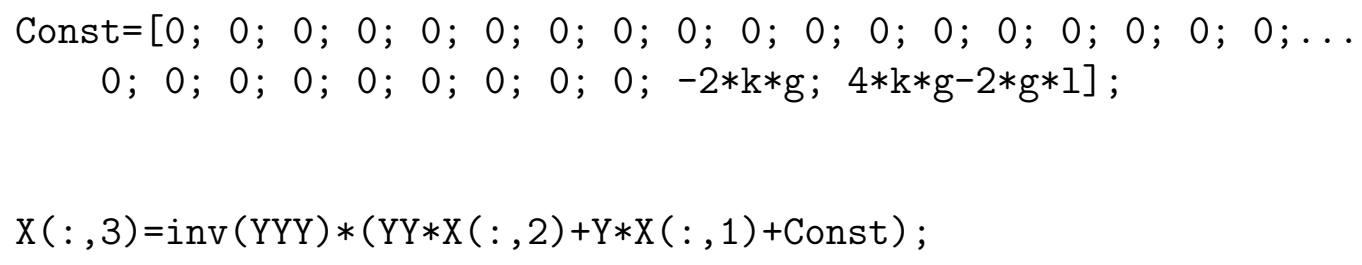




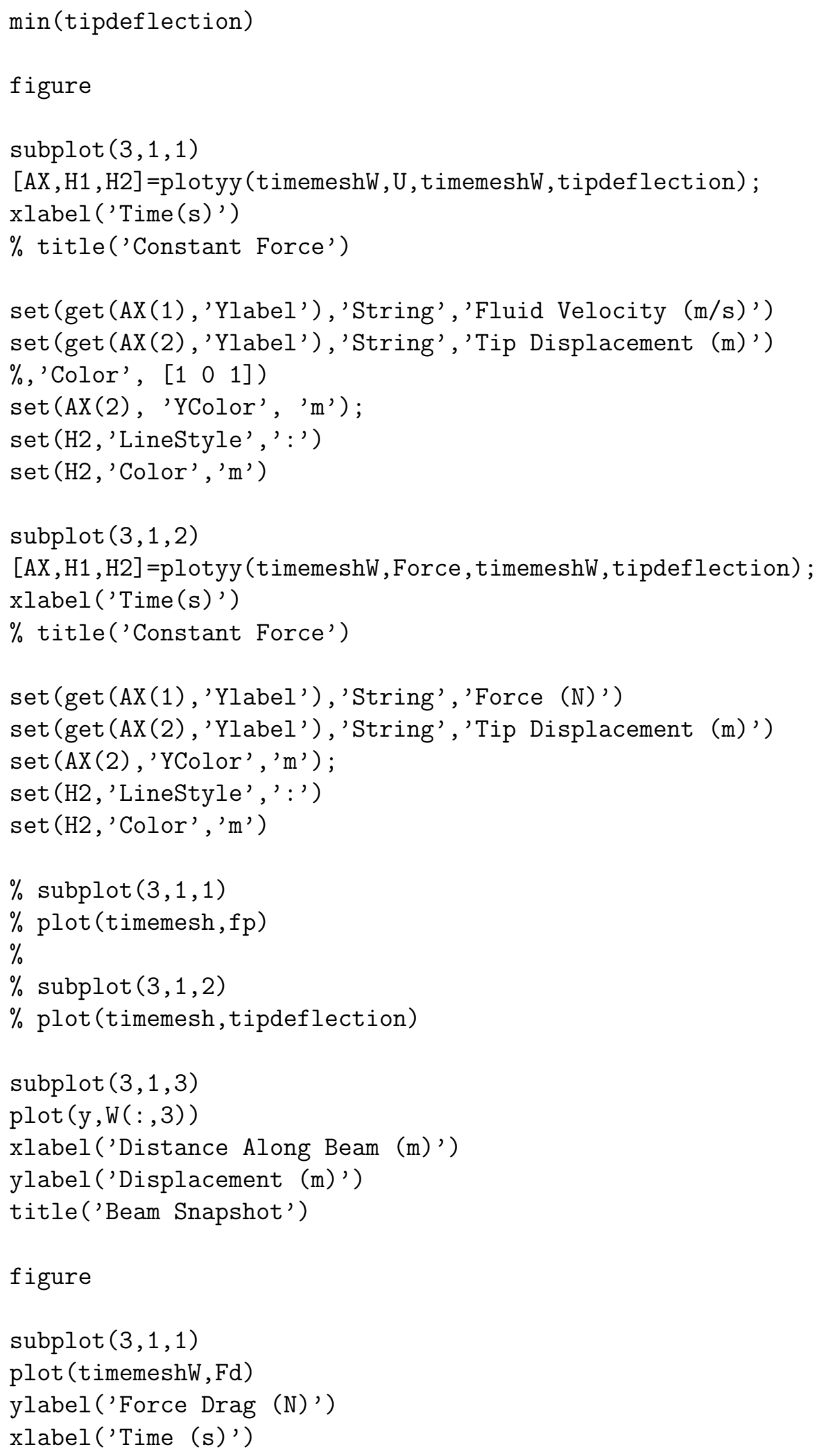




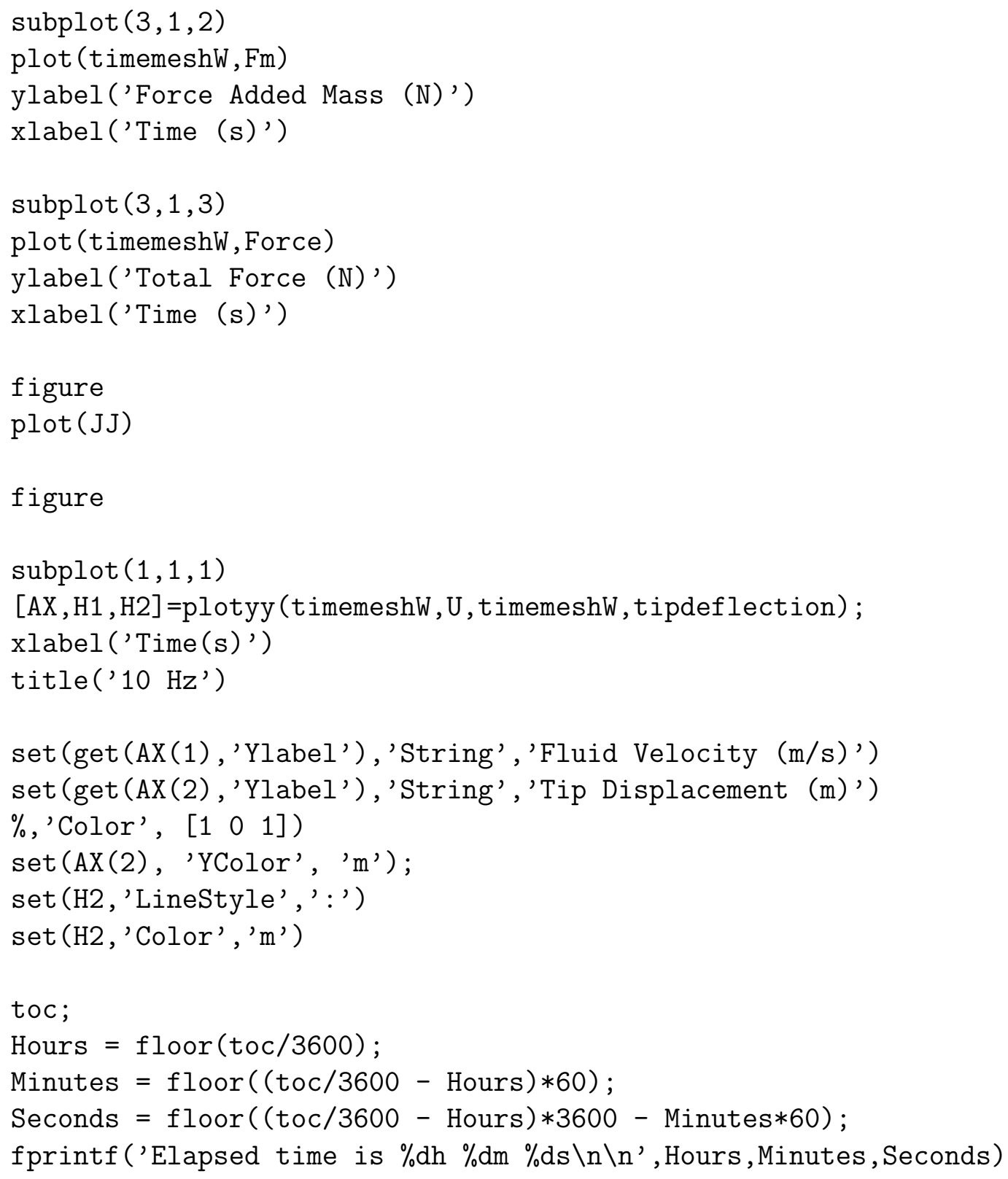




\section{Appendix $\mathrm{C}$}

\section{Flume Velocity Field}

The correlation of the motor propeller spin speed to the water flow in the test section is shown in Figure C.1. The slope of the line provides the input for the motor controller.

Examining the quality of the flow at $41.7 \mathrm{~cm} / \mathrm{s}$ or roughly half the maximum flow speed, the averge velocity field is shown in Figure C.2 with streamlines. Figure C.3 Shows the average turbulence intensity. Note the turbulence remains well below the $2 \%$ level quoted in the dissertation. 


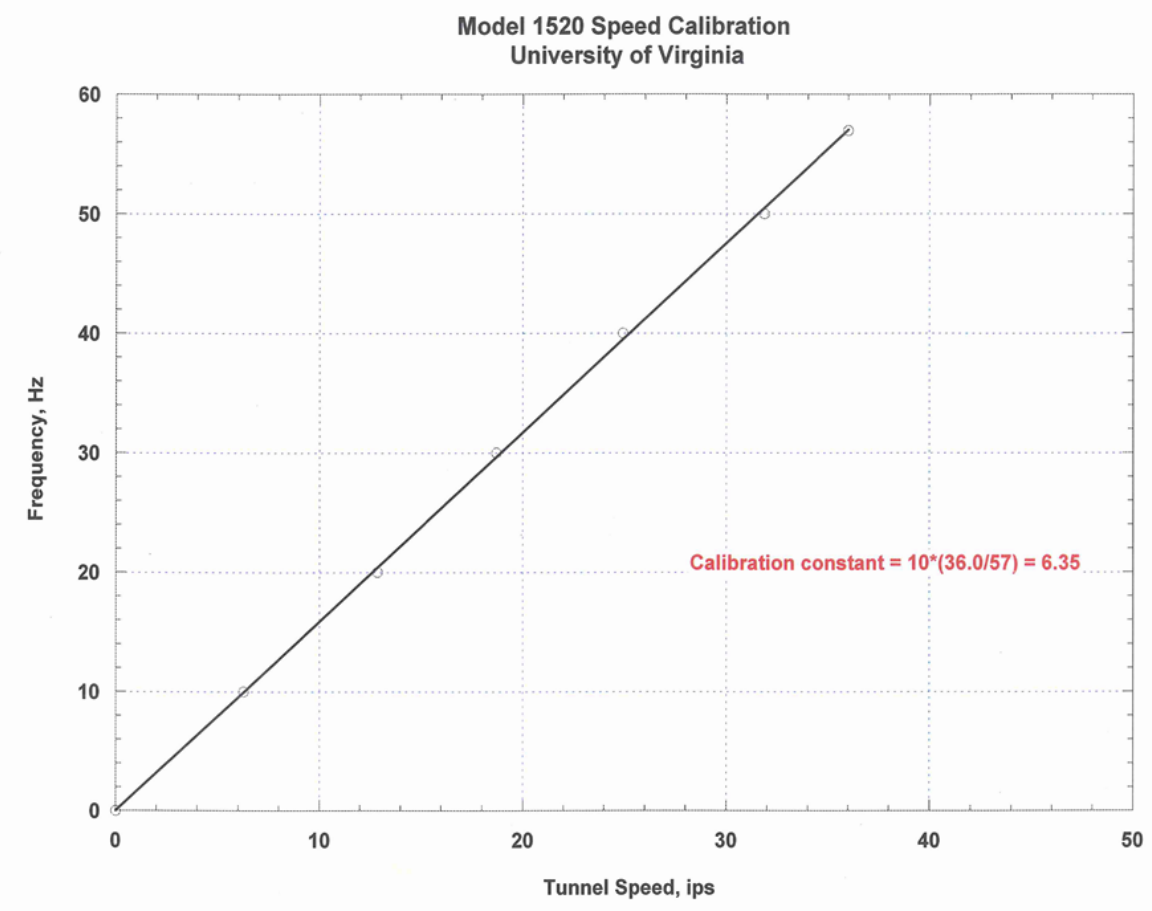

Figure C.1: Calibration of motor speed $(\mathrm{Hz})$ to water velocity (in/s)

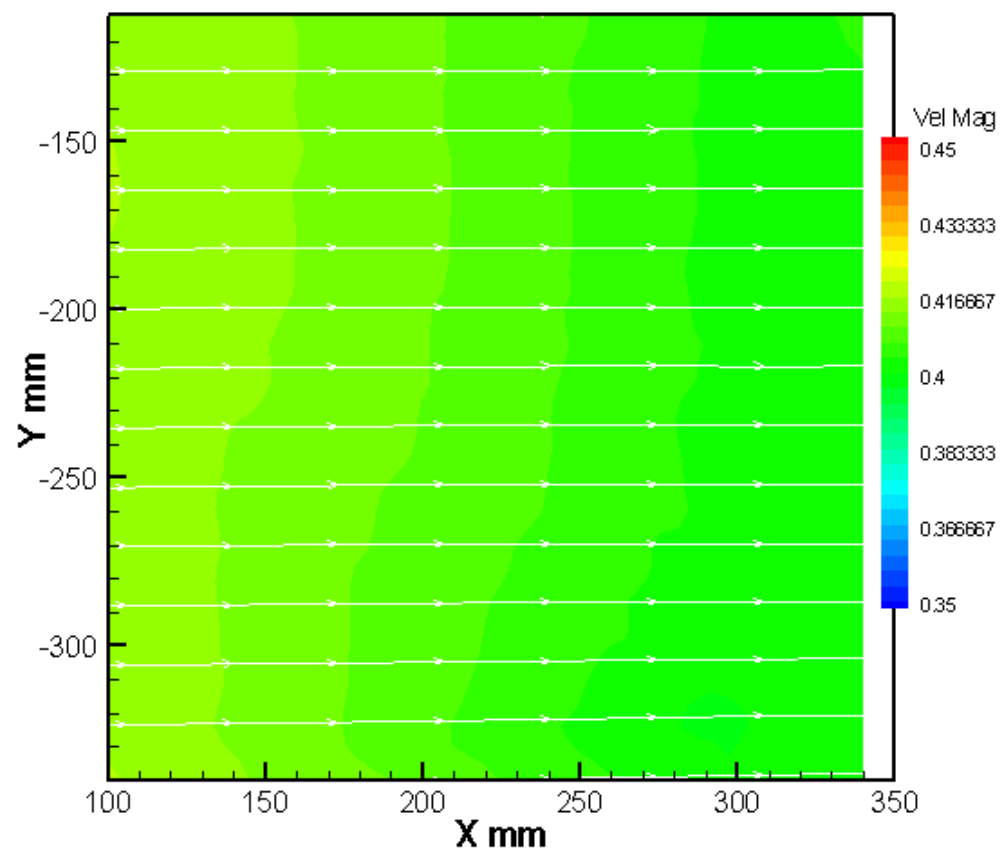

Figure C.2: Countour plot of flow velocity magnitude for $41.7 \mathrm{~cm} / \mathrm{s}$ flow. 


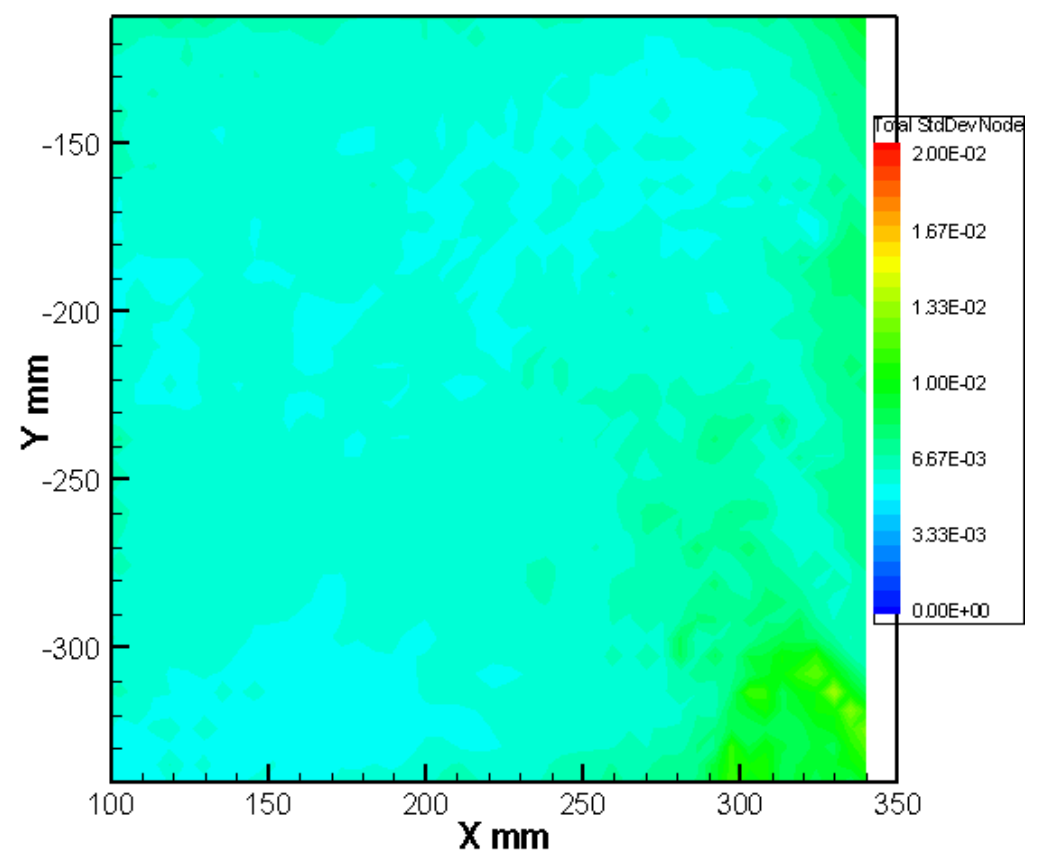

Figure C.3: Countour plot of flume turbulence for $41.7 \mathrm{~cm} / \mathrm{s}$ flow 